MICHELLY DE SOUZA

MODELAGEM, SIMULAÇÃO E FABRICAÇÃO DE CIRCUITOS ANALÓGICOS COM TRANSISTORES SOI CONVENCIONAIS E DE CANAL GRADUAL OPERANDO EM TEMPERATURAS CRIOGÊNICAS 
MICHELLY DE SOUZA

\section{MODELAGEM, SIMULAÇÃO E FABRICAÇÃO DE CIRCUITOS ANALÓGICOS COM TRANSISTORES SOI CONVENCIONAIS E DE CANAL GRADUAL OPERANDO EM TEMPERATURAS CRIOGÊNICAS}

Tese apresentada à Escola Politécnica da Universidade de São Paulo para a obtenção do título de Doutor em Engenharia.

Área de concentração: Microeletrônica.

Orientador: Prof. Dr. Marcelo Antonio Pavanello. 
Este exemplar foi revisado e alterado em relação à versão original, sob responsabilidade única do autor e com anuência de seu orientador.

São Paulo, 03 de Novembro de 2008.

Assinatura do autor

Assinatura do orientador

Souza, Michelly de

Modelagem, simulação e fabricação de circuitos analógicos com transistores SOI convencionais e de canal gradual operando em temperaturas criogênicas / M. de Souza. -- ed. rev. -- São Paulo, 2008.

$197 \mathrm{p}$.

Tese (Doutorado) - Escola Politécnica da Universidade de São Paulo. Departamento de Engenharia de Sistemas Eletrônicos.

1.Transistores (Modelagem) 2.Dispositivos eletrônicos 3.Circuitos analógicos 3.Medidas elétricas I.Universidade de São Paulo. Escola Politécnica. Departamento de Engenharia de Sistemas Eletrônicos II.t. 
Dedico este trabalho à minha família, a quem devo tudo o que sou e a quem muito amo. 


\section{AGRADECIMENTOS}

Ao Prof. Dr. Marcelo Antonio Pavanello, não somente pela dedicada e sempre presente orientação, mas também pela amizade e confiança depositada ao longo dos anos. O meu sincero agradecimento por ter contribuído para que eu me tornasse a pesquisadora que sou hoje.

Ao Prof. Dr. Denis Flandre, do Laboratório de Microeletrônica da Université catholique de Louvain, Bélgica, por ter me recebido em seu grupo de pesquisa, disponibilizando a infra-estrutura necessária para a fabricação de nossos transistores e circuitos, e pelas valiosas discussões e sugestões ao longo deste trabalho.

Ao Laboratório de Sistemas Integráveis da Escola Politécnica da Universidade de São Paulo, por disponibilizar a infra-estrutura para a realização de nossas atividades de pesquisa.

Ao Centro Universitário da $\mathrm{FEI}$, que gentilmente nos disponibilizou seu laboratório para a realização de medidas experimentais em baixas temperaturas.

Aos membros do grupo SOI-CMOS, pelo companheirismo durante estes anos e ao Prof. Dr. João Antonio Martino, por ter me dado a oportunidade de fazer parte deste grupo de pesquisa.

À minha mãe e irmã, Niljane e Gisely, pelo constante incentivo e apoio em todas as minhas escolhas, e pela compreensão nos muitos momentos de ausência.

Ao querido Marwin, por estar sempre ao meu lado, com seu apoio e dedicação, que certamente contribuíram para que este trabalho fosse possível.

À FAPESP, pelo apoio financeiro inestimável, que permitiu a realização deste trabalho.

A tantas outras pessoas, que de alguma forma colaboraram para a realização deste trabalho e que, de forma involuntária, foram aqui omitidas. 


\section{RESUMO}

Neste trabalho apresentamos a análise do comportamento analógico de transistores MOS implementados em tecnologia Silício sobre Isolante (SOI), de canal gradual (GC) e com tensão mecânica aplicada ao canal, operando em baixas temperaturas (de $380 \mathrm{~K}$ a $90 \mathrm{~K}$ ), em comparação com dispositivos SOI convencionais. Este estudo foi realizado utilizando-se medidas experimentais de transistores e pequenos circuitos fabricados, bem como através da utilização de simulações numéricas bidimensionais e modelos analíticos.

No caso dos transistores de canal gradual, inicialmente foi proposto um modelo analítico contínuo para a simulação da corrente de dreno em baixas temperaturas. Este modelo foi validado para temperaturas entre $300 \mathrm{~K} \mathrm{e} 100 \mathrm{~K} \mathrm{e}$ incluído na biblioteca de modelos de um simulador de circuitos. Foram analisadas características importantes para o funcionamento de circuitos analógicos, tais como a distorção harmônica de dispositivos operando em saturação e o descasamento de alguns parâmetros, como tensão de limiar e corrente de dreno, em diversas temperaturas. No caso da distorção, foi verificada uma melhora significativa promovida pela utilização da estrutura de canal gradual, ultrapassando $20 \mathrm{~dB}$ em 100 K. O descasamento apresentou piora em relação ao transistor convencional, devido a imperfeições de alinhamento que podem ocorrer no processo de fabricação, principalmente na etapa de definição da região fracamente dopada do canal. Foi observada uma piora de até $2,5 \mathrm{mV}$ na variação da tensão de limiar e mais de $2 \%$ na corrente de dreno, em temperatura ambiente, em relação ao transistor uniformemente dopado.

O impacto da utilização de transistores GC SOI em espelhos de corrente e amplificadores dreno comum também foi também avaliado. Os resultados experimentais mostraram que a estrutura de canal gradual é capaz de promover a melhora no desempenho destes dois blocos analógicos em comparação com transistores uniformemente dopados em todo o intervalo de temperaturas estudado. Amplificadores dreno comum com ganho praticamente constante e próximo do limite teórico e espelhos de corrente com precisão de espelhamento superior àquela 
apresentada por transistores convencionais, com maior excursão do sinal de saída e maior resistência de saída, foram obtidos.

Foram também comparadas características analógicas de transistores SOI com tensão mecânica uniaxial e biaxial agindo sobre o canal em função da temperatura. Os resultados obtidos indicam que a tensão mecânica sobre o canal resulta em ganho de tensão melhor, ou no mínimo igual, àquele obtido com um transistor convencional com as mesmas dimensões e tecnologia.

Palavras-chave: Tecnologia SOI. SOI nMOSFET de Canal Gradual. Transistores com canal tensionado. Circuitos Analógicos. 


\section{ABSTRACT}

In this work an analysis of the analog behavior of MOS transistors implemented in Silicon-on-Insulator technology, with graded-channel (GC) and mechanical strain applied to the channel, operating at low temperatures (from $380 \mathrm{~K}$ down to $90 \mathrm{~K}$ ), in comparison to standard SOI devices is presented. This study has been carried out by using experimental measurements of transistors and small circuits, as well as through two-dimensional numerical simulations and analytical models.

In the case of graded-channel transistors, an analytical model for the simulation of the drain current at low temperatures has been initially proposed. This model has been validated from $300 \mathrm{~K}$ down to $100 \mathrm{~K}$ and included to the models library of a circuit simulator. Important characteristics for analog circuits have been evaluated, namely the harmonic distortion of devices biased in saturation regime and the mismatching of parameters like the threshold voltage and the drain current, at several temperatures. Regarding the distortion, it has been verified a significant improvement due to the use of the graded-channel architecture, which reached more than $20 \mathrm{~dB}$ at $100 \mathrm{~K}$. The matching has been worsened in comparison to standard transistor, due to misalignements that may take place in the devices processing, mainly in the definition of the lightly doped region in the channel. It has been observed a worsening of up to $2.5 \mathrm{mV}$ in the threshold voltage variation and more than $2 \%$ in the drain current, at room temperature, in comparison to the uniformly doped device.

The impact of the application of GC transistors in current mirrors and commondrain amplifiers has been also evaluated. The experimental results showed that the graded-channel structure is able to provide improved performance of these analog blocks in comparison with uniformly doped transistors in the entire studied range of temperatures. Commom-drain amplifiers with virtually constant gain, close to the theoretical limit and current mirrors with improved mirroring precision in comparison to standard transistors, with increased output swing and output resistance have been obtained.

Analog characteristics of SOI transistors with uniaxial and biaxial mechanical strain in the channel have been also compared as a function of the temperature. The 
analysis of experimental measurements indicates that the use of mechanical strain results in better or, at least, similar voltage gain than stardard transistors, for the same dimensions and technology.

Keywords: SOI technology. Graded-Channel SOI nMOSFET. Strain SOI. Analog circuits. 


\section{LISTA DE FIGURAS}

Figura 1 - Perfil transversal de um transistor SOI nMOSFET. 38

Figura 2 - Diagramas de faixas de energia para transistores MOS convencional (A), SOI de camada espessa (B) e SOI de camada fina (C). 40

Figura 3 - Concentração intrínseca de portadores e largura da faixa proibida do silício em função da temperatura, calculadas através das eqs. (5) e (6)...45

Figura 4 - Porcentagem de impurezas ionizadas e potencial de Fermi em função da temperatura, para um substrato de silício tipo $p$, com $N_{A}=1.10^{17} \mathrm{~cm}^{-3} \ldots . .47$

Figura 5 - Curvas das diversas componentes que compõem a mobilidade de elétrons independente de campo elétrico em função da temperatura, para substrato de silício tipo $p$ dopado com $\mathrm{N}_{\mathrm{A}}=1.10^{17} \mathrm{~cm}^{-3}$

Figura 6 - Mobilidade de elétrons independente de campo elétrico em função da temperatura, para substrato de silício tipo $p$ e diferentes concentrações de dopantes.

Figura 7 - Transcondutância em função da tensão de porta, simulada para um dispositivo $\mathrm{SOI}$ totalmente depletado com $\mathrm{L}=0,5 \mu \mathrm{m}$ e $V_{D S}=100 \mathrm{mV}$, em diferentes temperaturas.

Figura 8 - Variação dos parâmetros $\alpha_{M}$ e $\beta_{M}$ com a temperatura ${ }^{69}$. 63

Figura 9 - Corrente de dreno (A) e condutância de dreno (B) em função da tensão de dreno, simuladas numericamente, com e sem o efeito de autoaquecimento.

Figura 10 - Amplificador de tensão com um único transistor MOS. 65

Figura 11 - Relação $g_{m} / I_{D S}$ em função de $I_{D S} /(W / L)$, simulada para transistores SOI totalmente depletados com $L=0,5 \mu \mathrm{m}$, operando em saturação $\left(V_{D S}=1,2\right.$ V), em diferentes temperaturas.

Figura 12 - Condutância de dreno em função da tensão de dreno, simulada para transistores SOI totalmente depletados com $L=0,5 \mu \mathrm{m}$, com $V_{\mathrm{GT}}=200$ $\mathrm{mV}$, em diferentes temperaturas. 68

Figura 13 - Perfil do transistor SOI MOSFET canal $n$ totalmente depletado com perfil de dopantes assimétrico na região de canal (GC SOI nMOSFET).

Figura 14 - Associação série de dois transistores SOI convencionais, representando 
o comportamento elétrico do transistor GC SOI.

Figura 15 - Comparação entre as curvas da corrente de dreno e transcondutância em função de $V_{\mathrm{GF}}$, modeladas (linhas) e medidas (símbolos), para todos os dispositivos operando em $\mathrm{T}=100 \mathrm{~K}$.

Figura 16 - Curvas da corrente de dreno e transcondutância em função de $\mathrm{V}_{\mathrm{GF}}$, modeladas (linhas) e medidas (símbolos), em diferentes temperaturas para o dispositivo GC SOI com $L_{L D} / L=0,28$.

Figura 17 - Curvas $I_{D S}$ em função de $V_{D S}(A)$ e $g_{D}$ em função de $V_{D S}(B)$, para dispositivos GC SOI com $L_{L D} / L=0,28$ medidas (símbolos) e modeladas (linhas) em diversas temperaturas com $\mathrm{V}_{\mathrm{GT}}=200 \mathrm{mV}$.

Figura 18 - Curvas $I_{D S}$ em função de $V_{D S}(A)$ e $g_{D}$ em função de $V_{D S}(B)$, para dispositivos GC SOI com $\mathrm{L}_{\mathrm{LD}} / \mathrm{L}=0,47$ medidas (símbolos) e modeladas (linhas) sob diversas condições de polarização de dreno, com T=300 K. 85

Figura 19 - Curvas $I_{D S}$ em função de $V_{D S}(A)$ e $g_{D}$ em função de $V_{D S}(B)$, para dispositivos GC SOI com $L_{L D} / L=0,47$ medidas (símbolos) e modeladas (linhas) sob diversas condições de polarização de dreno, com T=100 K. 85

Figura 20 - Comparação entre as curvas $I_{D s}$ em função de $V_{G F}$, com $V_{D S}=100 \mathrm{mV}$, obtidas com os simuladores Eldo (linhas) e Atlas (símbolos), de dispositivos SOI convencional (A) e GC SOI com $L_{L D} / L=0,5$ (B).

Figura 21 - Comparação entre as curvas da transcondutância em função de $V_{G F}$, com $V_{D S}=100 \mathrm{mV}$, obtidas com os simuladores Eldo (linhas) e Atlas (símbolos), para dispositivos SOI convencional (A) e GC SOI com $\mathrm{L}_{\mathrm{LD}} / \mathrm{L}=0,5(\mathrm{~B})$.......

Figura 22 - Comparação entre as curvas $I_{D S}$ e $g_{m}$ em função de $V_{G F}$, com $V_{D S}=100$ $\mathrm{mV}$, obtidas com os simuladores Eldo (linhas) e Atlas (símbolos), para todos os dispositivos estudados, com $\mathrm{T}=100 \mathrm{~K}$

Figura 23 - Comparação entre as curvas $I_{D S}$ em função de $V_{G F}$ com $V_{D S}=1,2 V$, obtidas com os simuladores Eldo (linhas) e Atlas (símbolos), para dispositivos GC SOI com $L_{L D} / L=0,5$ em diversas temperaturas. .90

Figura 24 - Comparação entre as curvas $\mathrm{g}_{\mathrm{m}} / \mathrm{l}_{\mathrm{DS}}$ em função de $\mathrm{IDS}_{\mathrm{DS}} /\left(\mathrm{W} / \mathrm{L}_{\text {eff }}\right)$, com $\mathrm{V}_{\mathrm{DS}}=1,2 \mathrm{~V}$, obtidas com os simuladores Eldo (linhas) e Atlas (símbolos), para dispositivos GC SOI com $\mathrm{L}_{\mathrm{LD}} / \mathrm{L}=0,5$ em diversas temperaturas.......91

Figura 25 - Comparação entre as curvas $I_{D S}$ em função de $V_{D S}$, com $V_{G T}=200 \mathrm{mV}$, obtidas com os simuladores Eldo (linhas) e Atlas (símbolos), de 
dispositivos e GC SOI com $\mathrm{L}_{\mathrm{LD}} / \mathrm{L}=0,4$ em diversas temperaturas.

Figura 26 - Comparação entre as curvas $I_{D s}$ e g em função de $V_{D S}$, com $V_{G T}=200$ $\mathrm{mV}$, obtidas com os simuladores Eldo (linhas) e Atlas (símbolos), para todos os dispositivos simulados, com $\mathrm{T}=100 \mathrm{~K}$. .92

Figura 27 - Transcondutância em função da sobretensão de condução de dispositivos com $L=0,5 \mu \mathrm{m}$ para diferentes valores de $V_{D s}$. 98

Figura 28 - Comparação entre as curvas experimentais (símbolos) e calculadas (linhas) da condutância de dreno em função da tensão de dreno, para diferentes dispositivos, com $V_{G T}=200 \mathrm{mV}$ (A) e para diferentes polarizações, para um transistor $\mathrm{GC}$ SOI com $\mathrm{L}_{\mathrm{LD}} / \mathrm{L}=0,28(\mathrm{~B})$. 100

Figura 29 - Ganho de tensão de malha aberta em função de $V_{G T}$ para dispositivos GC SOI e SOI convencional, polarizados com $V_{D S}=0,8$ e 1,5 V............100

Figura 30 - Leiaute do chip fabricado 103

Figura 31 - Fotos dos espelhos de corrente fabricados, com estruturas Cascode (A) e Wilson (B). 104

Figura 32 - Fotos de uma das estruturas para estudo de descasamento. 104

Figura 33 - Curvas $I_{D S}$ em função de $V_{G T}$ obtidas através de medidas (símbolos) e do modelo analítico (linhas), com $V_{D S}=1,5 \mathrm{~V}$ 106

Figura 34 - Tensão Early e ganho de tensão de malha aberta em função da relação $\mathrm{g}_{\mathrm{m}} / \mathrm{l}_{\mathrm{DS}}$ (valores medidos). 107

Figura 35 - Comparação entre as curvas THD/A $(A)$ e HD2/A $A_{v}$ e HD3/Av $(B)$ obtidas a partir de dados experimentais (símbolos) e modelados (linhas), com $\mathrm{Va}=50 \mathrm{mV}$. 108

Figura $36-\mathrm{THD} \mathrm{A}_{V}(\mathrm{~A})$ e HD3/AV $(B)$ obtidos através de dados experimentais (símbolos) e do modelo analítico (linhas), variando $\mathrm{Va}$ com $\mathrm{g}_{\mathrm{m}} / \mathrm{l}_{\mathrm{DS}}=5 \mathrm{~V}^{-1}$.

Figura 37 - THD/Av em função de $g_{m} / l_{D S}$ para dispositivos GC SOI e SOI convencional operando em $300 \mathrm{~K}(\mathrm{~A})$ e $100 \mathrm{~K}(\mathrm{~B})$, com $\mathrm{V}_{\mathrm{DS}}=1,1 \mathrm{~V}$ e $\mathrm{Va}=50 \mathrm{mV}$

Figura 38 - Curvas $g_{m}{ }^{2}$ e $d g_{m} / d V_{G F}$ em função de $g_{m} / l_{D S}$ obtidas através das curvas modeladas de dispositivos $\mathrm{SOI}$ convencional e GC SOI com $\mathrm{L}_{\mathrm{LD}} / \mathrm{L}=0,28$ com $V_{D S}=1,1 \mathrm{~V}$ em $T=100 \mathrm{~K}$ e $300 \mathrm{~K}$

Figura 39 - Curvas da condutância de dreno em função de $\mathrm{g}_{\mathrm{m}} / \mathrm{I}_{\mathrm{DS}}$ obtidas através das curvas modeladas de dispositivos SOI convencional e GC SOI com 
$\mathrm{L}_{\mathrm{LD}} / \mathrm{L}=0,28, \mathrm{com} \mathrm{V}_{\mathrm{DS}}=1,1 \mathrm{~V}$ em $\mathrm{T}=100 \mathrm{~K}$ e $300 \mathrm{~K}$ 113

Figura $40-\sigma_{\mathrm{V}_{\text {thf }}} / \mathrm{V}_{\text {thf,mean }}$ em função da relação $1 / \sqrt{\mathrm{W} \times \mathrm{L}_{\text {eff }}}$ para transistores de canal gradual com diversas relações $\mathrm{L}_{\mathrm{LD}} / \mathrm{L}$.

Figura 41 - Desvio padrão da corrente de dreno em função da sobretensão de condução com baixa tensão aplicada ao dreno $\left(\mathrm{V}_{\mathrm{DS}}=25 \mathrm{mV}\right)$ obtido experimentalmente. 119

Figura 42 - Desvio padrão da corrente de dreno obtida através do modelo analítico e de seus termos $\beta$ e $\mathrm{k}_{\mathrm{Q}}$ em função da tensão aplicada à porta com $\mathrm{V}_{\mathrm{DS}}=100 \mathrm{mV}$. 121

Figura 43 - Desvio padrão da corrente de dreno obtida através do modelo analítico e de seus termos $\beta$ e $k_{Q}$ em função da corrente de dreno normalizada, em saturação $\left(\mathrm{V}_{\mathrm{DS}}=1,5 \mathrm{~V}\right)$.

Figura 44 - Desvio padrão da tensão de saturação obtida através do modelo analítico em função da corrente de dreno normalizada, em saturação $\left(\mathrm{V}_{\mathrm{DS}}=1,5 \mathrm{~V}\right)$.

Figura 45 - Comparação entre o desvio padrão da corrente de dreno medida e simulada em função da sobretensão de condução em região linear $\left(\mathrm{V}_{\mathrm{DS}}=25 \mathrm{mV}\right)$

Figura 46 - Desvio padrão da corrente de dreno simulada em saturação $\left(\mathrm{V}_{D S}=1,5 \mathrm{~V}\right)$ em função da corrente de dreno normalizada.

Figura 47 - Valor médio da tensão de limiar em função do comprimento efetivo de canal para diferentes temperaturas. 126

Figura 48 - Desvio padrão da tensão de limiar em função do comprimento efetivo de canal medido em diferentes temperaturas. 127

Figura 49 - Desvio padrão da porcentagem de impurezas ionizadas em função da temperatura, calculados a partir das equações apresentadas na seção 2.2, considerando diferentes concentrações de dopantes, $\mathrm{N}_{\mathrm{A}}$, e impondo variação de $\pm 10 \%$. 128

Figura $50-\sigma_{V_{\text {thf }}} / V_{\text {thf,mean }}$ em função do comprimento efetivo de canal, medido para diferentes temperaturas. 129

Figura 51 - Variação da corrente de dreno em função da corrente de dreno normalizada, medida para transistores SOI convencional e GC SOI com $L_{L D} / L=0,24$ em saturação $\left(V_{D S}=1,5 \mathrm{~V}\right)$. 
Figura 52 - Transistor configurado como amplificador dreno comum ou seguidor de fonte.

Figura 53 - Curvas $V_{\text {OUT }}$ e $A_{V}$ em função de $V_{I N}$ de seguidores de fonte implementados com transistores SOI convencional e de canal gradual, medidas em inversão fraca $(A)$ e forte $(B)$. 133

Figura 54 - Modelo equivalente de pequenos sinais para o seguidor de fonte. 134

Figura 55 -Transcondutância em função da tensão de entrada, extraída a partir de curvas $I_{D S}$ em função de $V_{G F}$ experimentais, medidas com $V_{D D}=2,5 \mathrm{~V}$ e $\mathrm{V}_{\mathrm{S}}=0 \mathrm{~V}$ 136

Figura 56 - Condutância de saída experimental extraída para $\mathrm{V}_{\mathrm{DD}}=1,5 \mathrm{~V}$ (símbolos abertos) e $2,5 \mathrm{~V}$ (símbolos fechados), com $\mathrm{V}_{\mathrm{S}}=0 \mathrm{~V}$. 136

Figura 57 - Fator 1/n obtido experimentalmente e $A_{\vee}$ calculado, em função da tensão de entrada com $V_{D D}=1,5 \mathrm{~V}$ 138

Figura 58 - Distorção harmônica total (THD) em função de $V_{\mathbb{I N}}$, for seguidores de fonte polarizados em inversão fraca $(A)$ e forte $(B)$, com $V_{D D}=2,5 \vee$ e $\mathrm{Va}=50 \mathrm{mV}$ 139

Figura 59 - Ganho de tensão em função da tensão de entrada simulado para seguidores de fonte com transistores convencional e de canal gradual ( $L=2 \mu \mathrm{m})$, com (linhas) e sem (símbolos) o modelo de ionização por impacto, em inversão fraca $(A)$ e forte $(B)$, com $V_{D D}=2,5 \mathrm{~V}$.

Figura 60 - Ganho experimental de seguidores de fonte implementados com transistores SOI convencionais e de canal gradual com diferentes comprimentos de canal, polarizados em inversão fraca $(A)$ e forte $(B)$, com $\mathrm{I}_{\text {bias }} /\left(\mathrm{W} / \mathrm{L}_{\text {eff }}\right)=0,1 \mathrm{nA}$ e $\quad 4,0 \mu \mathrm{A}$, respectivamente, e $\mathrm{V}_{\mathrm{DD}}=2,5 \mathrm{~V}$. 144

Figura 61 - Distorção harmônica total em função de $V_{\mathbb{I N}}$ para seguidores de fonte operando em inversão forte, polarizados com $\mathrm{I}_{\text {bias }} /\left(\mathrm{W} / \mathrm{L}_{\text {eff }}\right)$ e $\mathrm{V}_{\mathrm{DD}}=2,5 \mathrm{~V}$, considerando $\mathrm{Va}=50 \mathrm{mV}$

Figura 62 - Curvas $A_{V}$ em função de $V_{\mathbb{I N}}$ simuladas para seguidores de fonte implementados com transistores convencionais e GC SOI com $L_{L D} / L=0,2$ e diferentes comprimentos de canal, em inversão fraca $(A)$ e forte $(B)$, com $I_{\text {bias }} /\left(\mathrm{W} / L_{\text {eff }}\right)=0,1 \mathrm{nA}$ e 4,0 $\mu \mathrm{A}$, com $\mathrm{V}_{\mathrm{DD}}=2,5 \mathrm{~V}$. 148

Figura 63 - THD em função de $V_{\mathbb{I N}}$ extraídas das curvas $V_{\text {OUT }}$ em função de $V_{\mathbb{I N}}$ simuladas em inversão forte, para transistores convencionais e de canal 
gradual com diferentes comprimentos de canal, considerando $\mathrm{Va}=50 \mathrm{mV}$.

Figura 64 - Curvas $\mathrm{V}_{\text {OUT }}$ em função de $\mathrm{V}_{\mathrm{IN}}$ de seguidores de fonte implementados com transistores convencional e GC SOI com $L=2 \mu \mathrm{m}$, medidas em inversão fraca (A) e forte (B), em $90 \mathrm{~K} \mathrm{e} 300 \mathrm{~K}$ 150

Figura 65 - Ganho em função da tensão de entrada, medidos para seguidores de fonte implementados com transistores convencional e GC SOI com $L=2$ $\mu \mathrm{m}$, medidas em inversão fraca (A) e forte (B), em $90 \mathrm{~K}$ e $300 \mathrm{~K}$. 151

Figura 66 - Ganho de tensão para transistores convencional e GC SOI com LLD/L= 0,2 , configurados como amplificador dreno comum operando em inversão forte, para diferentes temperaturas. 152

Figura 67 - Representação esquemática de espelhos de corrente nas arquiteturas Wilson (A) e Cascode (B). 155

Figura 68 - Precisão de espelhamento em função da corrente de entrada normalizada, obtidas experimentalmente, para espelhos de corrente nas arquiteturas Wilson (A) e Cascode (B), com a temperatura variando de $380 \mathrm{~K}$ a $150 \mathrm{~K}$ 156

Figura 69 - Curvas lout em função de $V_{D, O U t}$ medidas para espelhos de corrente Wilson (A) e Cascode (B), com transistores SOI convencionais e de canal gradual, em $T=150 \mathrm{~K}$ e $300 \mathrm{~K}$. 158

Figura 70 - Tensão de saturação e de ruptura em função da temperatura, extraídas a partir das curvas $\mathrm{I}_{\mathrm{OUT}}$ em função de $\mathrm{V}_{\mathrm{D}, \mathrm{OUT}}$, com $\mathrm{I}_{\mathrm{N}}=1 \mu \mathrm{A}$. 159

Figura 71 - Excursão de saída em função da temperatura extraída de espelhos de corrente implementados com transistores GC SOI e convencionais, nas arquiteturas Wilson (A) e Cascode (B). 160

Figura 72 - Resistência de saída em função da temperatura para espelhos de corrente Wilson (A) e Cascode (B) implementados com transistores convencionais e de canal gradual. 161

Figura 73 - Corrente de dreno, porta e corrente de dreno intrínseca na ausência da corrente de porta, todas em função da tensão de porta, para um transistor SOI nMOSFET convencional, com $\mathrm{L}=10 \mu \mathrm{m}$ e $\mathrm{W}=1 \mu \mathrm{m}$, polarizado com $\mathrm{V}_{\mathrm{DS}}=50 \mathrm{mV}$. 164

Figura 74 - Corrente de dreno $(A)$ e transcondutância $(B)$ em função da sobretensão 
de condução obtida com $\mathrm{V}_{\mathrm{DS}}=50 \mathrm{mV}$ para dispositivos com $\mathrm{L}=160 \mathrm{~nm}$ com e sem sCESL, em $150 \mathrm{~K}$ e $300 \mathrm{~K}$.

Figura 75 - Transcondutância máxima em função da temperatura para transistores com e sem SCESL com diferentes comprimentos de canal 166

Figura 76 - Transcondutância máxima, obtida em região linear, em função do comprimento de canal com T=100 K, $200 \mathrm{~K}$ e $300 \mathrm{~K}$. 166

Figura 77 - Ganho de transcondutância proporcionado pelo sCESL, em relação ao SOI convencional, em função da temperatura, para dispositivos com $\mathrm{L}=160 \mathrm{~nm}$ e $910 \mathrm{~nm}$. 167

Figura 78 - Inclinação de sublimiar em função da temperatura para dispositivos com $\mathrm{L}=160 \mathrm{~nm}$. 168

Figura 79 - Transcondutância máxima em função da temperatura, extraída para transistores com e sem sCESL, com $V_{D S}=0,55 \mathrm{~V}$ para diferentes comprimentos de canal. 168

Figura 80 - Condutância de dreno em função da temperatura, extraída para dispositivos com $L=160 \mathrm{~nm}$, com $V_{D S}=0,55 \mathrm{~V}$ e diferentes valores de $V_{G T}$. 169

Figura 81 - Ganho de tensão intrínseco de dispositivos com L=160 nm, obtido com $V_{D S}=0,55 V$ e $g_{m} / I_{D S}=3 V^{-1}$, e $V_{G T}$ necessário, em função da temperatura. 170

Figura $82-T H D / A_{v}$ e HD3/Av em função de $g_{m} / I_{D s}$ para dispositivos com $L=160 \mathrm{~nm}$ com $\mathrm{V}_{\mathrm{DS}}=0,55 \mathrm{~V}$ e $\mathrm{Va}=50 \mathrm{mV}$

Figura 83 - Transcondutância máxima em função da temperatura, extraída com $\mathrm{V}_{\mathrm{DS}}=100 \mathrm{mV}$ e $550 \mathrm{mV}$ para transistores com e sem tensão mecânica $(\mathrm{L}=160 \mu \mathrm{m})$. 172

Figura 84 - Relação $\mathrm{g}_{\mathrm{m}} / \mathrm{I}_{\mathrm{DS}}$ em função da corrente de dreno normalizada, medida para transistores convencional e com tensão mecânica, com $L=160$ nm, em diversas temperaturas.

Figura 85 - Condutância de saída e ganho de tensão em função da temperatura, extraídos com $\mathrm{V}_{\mathrm{DS}}=550 \mathrm{mV}$ e $\mathrm{V}_{\mathrm{GT}}=400 \mathrm{mV}$ 


\section{LISTA DE TABELAS}

Tabela 1 - Parâmetros de modelo utilizados na comparação com curvas experimentais obtidas para transistores GC SOI com $L_{L D} / L=0,28$ e 0,47 em 300 e $100 \mathrm{~K}$.

Tabela 2 - Ganho intrínseco obtido através do modelo analítico e de dados experimentais, para os dispositivos $\mathrm{GC}$ com $\mathrm{L}_{\mathrm{LD}} / \mathrm{L}=0,28$, polarizados com

Tabela 3 - Parâmetros de modelo utilizados na comparação com curvas obtidas através do simulador numérico para transistores convencional e GC SOI com $L_{L D} / L=0,50$, em $300 \mathrm{~K}$ e $100 \mathrm{~K}$.

Tabela 4 - Ganho de malha aberta, obtido com $\mathrm{V}_{\mathrm{GT}}=200 \mathrm{mV}$ e $\mathrm{V}_{\mathrm{DS}}=1,5 \mathrm{~V}$.........106

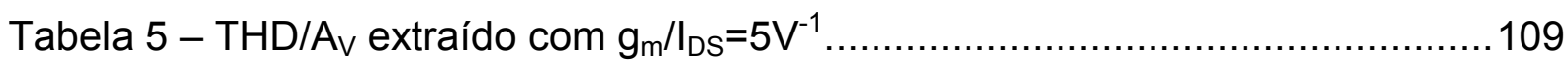

Tabela 6 - Valor médio e desvio padrão da tensão de limiar de transistores SOI convencional e GC SOI. ........................................................... 115

Tabela 7 - Valores de $\sigma_{0 V_{\text {thf }}}$ e $\sigma_{0 V_{\text {thf }}} / V_{\text {thf,mean }}$ calculados a partir dos dados experimentais apresentados na Figura 40.

Tabela 8 - Fator de corpo (n) extraído através da inclinação de sublimiar de curvas $I_{D S}$ em função de $V_{G F}$ experimentais, com $V_{D S}=50 \mathrm{mV}$ and $1,5 \mathrm{~V}$.

Tabela 9 - Fator de corpo (n) extraído através da inclinação de sublimiar de curvas $\mathrm{I}_{\mathrm{DS}}$ em função de $\mathrm{V}_{\mathrm{GF}}$ experimentais, com $\mathrm{V}_{\mathrm{DS}}=50 \mathrm{mV}$ and $1,5 \mathrm{~V}$, para transistores SOI convencionais e de canal gradual para diferentes comprimentos de canal. 145

Tabela 10 - Valores simulados de $g_{m}$, $g_{D}$ e $k$ e sua variação com a temperatura, $e$ ganho calculado, extraídos com $\mathrm{V}_{\mathrm{IN}}=\mathrm{V}_{\mathrm{DS}}=1 \mathrm{~V}$ para seguidores de fonte implementados com transistores convencional e GC SOI com $L_{L D} / L=0,20$.

Tabela 11 - DIBL extraído com $V_{D S}=100 \mathrm{mV}$ e $1 \mathrm{~V}$ para transistores com $\mathrm{L}=160 \mathrm{~nm}$ em diferentes temperaturas. 


\section{LISTA DE ABREVIATURAS E SIGLAS}

$\begin{array}{ll}\text { CMOS } & \text { Complementary Metal-Oxide-Semiconductor } \\ \text { DIBL } & \text { Drain-Induced Barrier Lowering } \\ \text { FD } & \text { Fully depleted } \\ \text { GC } & \text { Graded-Channel } \\ \text { HD2 } & \text { Second Order Harmonic Distortion } \\ \text { HD3 } & \text { Third Order Harmonic Distortion } \\ \text { IFM } & \text { Integral Function Method } \\ \text { IMEC } & \text { Interuniversity Microelectronics Center } \\ \text { HD } & \text { Highly Doped } \\ \text { LD } & \text { Lightly Doped } \\ \text { LDD } & \text { Lightly Doped Drain } \\ \text { LPLV } & \text { Low-power Low-voltage } \\ \text { MOSFET } & \text { Metal-Oxide-Semiconductor Field-Effect Transistor } \\ \text { NFD } & \text { Near-fully depleted } \\ \text { PD } & \text { Partially Depleted } \\ \text { SCESL } & \text { Strained Contact-Etch Stop Layer } \\ \text { SOI } & \text { Silicon-On-Insular } \\ \text { SRB } & \text { Stress Relaxed Buffer } \\ \text { SSOI } & \text { Strained Silicon-On-Insulator } \\ \text { THD } & \text { Total Harmonic Distortion } \\ \text { UCL } & \text { User Definable Models } \\ \text { UDM } & \text { Ultra Large Scale Integration } \\ \text { ULSI } & \text { ZTC }\end{array}$




\section{LISTA DE SÍMBOLOS}

A

$\mathrm{A}_{T S}$

$A_{V}$

$\mathrm{B}_{\text {TS }}$

$\mathrm{BV}_{\mathrm{CM}}$

$B V_{D S}$

$\mathrm{C}_{b b}$

$\mathrm{C}_{\mathrm{D}}$

$\mathrm{C}_{\text {itb }}$

$\mathrm{C}_{\text {iff }}$

$C_{L}$

$\mathrm{C}_{\text {ox }}$

$\mathrm{C}_{\text {oxb }}$

$\mathrm{C}_{\text {oxf }}$

$\mathrm{C}_{\mathrm{Si}}$

$\mathrm{E}_{\mathrm{A}}$

$\mathrm{E}_{\mathrm{C}}$

$E_{D}$

$E_{\text {eff }}$

$\mathrm{E}_{\mathrm{F}}$
Parâmetro de ajuste para o cálculo da corrente gerada por ionização por impacto

Parâmetro que controla a transição entre as regiões de triodo e saturação

Ganho de tensão de malha aberta em baixa freqüência

Parâmetro que controla a transição entre as regiões de triodo e saturação

Tensão de ruptura do espelho de corrente [V]

Tensão de ruptura de dreno [V]

Parâmetro resultante da associação de capacitâncias do transistor SOI de camada fina $\left[\mathrm{F} / \mathrm{cm}^{2}\right]$

Capacitância da região de depleção por unidade de área $\left[\mathrm{F} / \mathrm{cm}^{2}\right]$

Capacitância das armadilhas de interface na segunda interface por unidade de área $\left[\mathrm{F} / \mathrm{cm}^{2}\right]$

Capacitância das armadilhas de interface na primeira interface por unidade de área $\left[\mathrm{F} / \mathrm{cm}^{2}\right]$

Capacitância de carga [F]

Capacitância do óxido de porta do transistor MOS por unidade de área $\left[\mathrm{F} / \mathrm{cm}^{2}\right]$

Capacitância do óxido enterrado por unidade de área $\left[\mathrm{F} / \mathrm{cm}^{2}\right]$

Capacitância do óxido de porta do transistor SOI por unidade de área $\left[\mathrm{F} / \mathrm{cm}^{2}\right]$

Capacitância da camada de silício por unidade de área $\left[\mathrm{F} / \mathrm{cm}^{2}\right]$

Nível de energia das impurezas aceitadoras [eV]

Nível de energia inferior da faixa de condução [eV]

Nível de energia das impurezas doadoras $[\mathrm{eV}]$

Campo elétrico normal médio no canal nas direções vertical e lateral $[\mathrm{V} / \mathrm{cm}]$

Nível de Fermi do semicondutor [eV] 


\begin{tabular}{|c|c|}
\hline$E_{F B}$ & Nível de Fermi do substrato [eV] \\
\hline $\mathrm{E}_{\mathrm{FM}}$ & Nível de Fermi do metal/eletrodo de porta [eV] \\
\hline $\mathrm{E}_{\mathrm{Fn}}$ & Nível de Fermi para elétrons [eV] \\
\hline $\mathrm{E}_{\mathrm{Fp}}$ & Nível de Fermi para lacunas [eV] \\
\hline Eg & Largura da faixa proibida $[\mathrm{eV}]$ \\
\hline $\mathrm{E}_{\mathrm{i}}$ & Nível intrínseco [eV] \\
\hline $\mathrm{E}_{\mathrm{V}}$ & Nível de energia superior da faixa de valência [eV] \\
\hline$g_{D}$ & Condutância de dreno [S] \\
\hline gDsat & Condutância de dreno na região de saturação [S] \\
\hline $\mathrm{gm}_{\mathrm{m}}$ & Transcondutância do transistor [S] \\
\hline $\mathrm{g}_{\mathrm{m} 3}$ & $\begin{array}{l}\text { Transcondutância do transistor Q3 dos espelhos de corrente } \\
\text { (Figura 67) [S] }\end{array}$ \\
\hline$g_{m, \max }$ & Transcondutância máxima do transistor [S] \\
\hline$g_{m} / l_{D S}$ & $\begin{array}{l}\text { Relação entre a transcondutância e a corrente de dreno do } \\
\text { transistor MOS }\left[\mathrm{V}^{-1}\right]\end{array}$ \\
\hline$g_{\mathrm{ms}}$ & Transcondutância de fonte do transistor [S] \\
\hline h & Constante de Planck $\left[6,63 \times 10^{-34} \mathrm{~J} . \mathrm{s}\right]$ \\
\hline HD2 & Distorção do harmônico de segunda ordem [dB] \\
\hline HD3 & Distorção do harmônico de terceira ordem [dB] \\
\hline$I_{\text {bias }}$ & $\begin{array}{l}\text { Corrente constante de polarização fornecida pela fonte de } \\
\text { corrente }[A]\end{array}$ \\
\hline$i_{C}$ & Coeficiente que indica o nível de inversão do transistor \\
\hline $\mathrm{I}_{\mathrm{D}}$ & Corrente de dreno $[\mathrm{A}]$ \\
\hline $\mathrm{I}_{\mathrm{D} 0}$ & $\begin{array}{l}\text { Corrente de dreno intrínseca, na ausência de corrente de porta } \\
\text { [A] }\end{array}$ \\
\hline IDS & Corrente entre dreno e fonte $[\mathrm{A}]$ \\
\hline $\mathrm{I}_{\mathrm{DS}} /\left(\mathrm{W} / \mathrm{L}_{\mathrm{eff}}\right)$ & Corrente normalizada entre dreno e fonte $[\mathrm{A}]$ \\
\hline IDS,mean & Corrente de dreno média $[A]$ \\
\hline IDSsat & Corrente de saturação entre dreno e fonte $[\mathrm{A}]$ \\
\hline
\end{tabular}




\begin{tabular}{|c|c|}
\hline IDS,total & $\begin{array}{l}\text { Corrente de dreno total, considerando o efeito de ionização por } \\
\text { impacto }[A]\end{array}$ \\
\hline$I_{G}$ & Corrente de porta $[\mathrm{A}]$ \\
\hline$I_{G D}$ & Corrente entre porta e dreno $[\mathrm{A}]$ \\
\hline $\mathrm{I}_{\mathrm{i}}$ & Corrente gerada por ionização por impacto $[\mathrm{A}]$ \\
\hline$l_{\mathrm{IN}}$ & Corrente no ramo de entrada do espelho de corrente $[\mathrm{A}]$ \\
\hline$I_{n}$ & Corrente de dreno normalizada $[\mathrm{A}]$ \\
\hline lout & Corrente no ramo de saída do espelho de corrente $[A]$ \\
\hline $\mathrm{I}_{\mathrm{s}}$ & Corrente de normalização $[\mathrm{A}]$ \\
\hline $\mathrm{k}$ & Constante de Boltzmann $\left[1,38066 \times 10^{-23} \mathrm{~J} / \mathrm{K}\right]$ \\
\hline $\mathrm{k}_{\mathrm{Q}}$ & $\begin{array}{l}\text { Termo dependente das cargas de inversão, utilizado no cálculo } \\
\text { da corrente de dreno }\end{array}$ \\
\hline $\mathrm{k}_{\mathrm{Q} \text {,mean }}$ & $\begin{array}{l}\text { Valor médio do termo dependente das cargas de inversão, } \\
\text { utilizado no cálculo da corrente de dreno }\end{array}$ \\
\hline L & Comprimento de máscara do canal do transistor $[\mu \mathrm{m}]$ \\
\hline $\mathrm{I}_{\mathrm{c}}$ & Comprimento característico $[\mu \mathrm{m}]$ \\
\hline$L_{\text {eff }}$ & Comprimento efetivo do canal do transistor $[\mu \mathrm{m}]$ \\
\hline $\mathrm{L}_{H D}$ & $\begin{array}{l}\text { Comprimento da região fortemente dopada no transistor GC SOI } \\
{[\mu \mathrm{m}]}\end{array}$ \\
\hline LLD & $\begin{array}{l}\text { Comprimento da região fracamente dopada no transistor GC SOI } \\
{[\mu \mathrm{m}]}\end{array}$ \\
\hline LLD/L & $\begin{array}{l}\text { Relação entre o comprimento da região fracamente dopada e o } \\
\text { comprimento de canal, no transistor GC SOI }\end{array}$ \\
\hline$L_{\text {sat }}$ & Comprimento da região saturada $[\mu \mathrm{m}]$ \\
\hline M & $\begin{array}{l}\text { Fator de multiplicação de corrente devido ao elevado campo } \\
\text { elétrico }\end{array}$ \\
\hline $\mathrm{m}_{0}$ & Massa do elétron $\left[9,11 \times 10^{-31} \mathrm{~kg}\right]$ \\
\hline$m_{\mathrm{ce}}{ }^{*}$ & Massa efetiva de condução para elétrons \\
\hline $\mathrm{m}_{\mathrm{ch}}{ }^{*}$ & Massa efetiva de condução para lacunas \\
\hline$m_{d e}{ }^{*}$ & Massa efetiva de densidade de estados para elétrons \\
\hline
\end{tabular}



$\mathrm{m}_{\mathrm{dh}}{ }^{*}$
Massa efetiva de densidade de estados para lacunas
Fator de corpo
$\mathrm{N}_{\mathrm{A}}$
Concentração de impurezas aceitadoras em um semicondutor $\left[\mathrm{cm}^{-3}\right]$
$\mathrm{N}_{\mathrm{A}}^{-} \quad$ Concentração de impurezas aceitadoras ionizadas em um semicondutor $\left[\mathrm{cm}^{-3}\right]$
$\mathrm{N}_{\mathrm{A}, \text { eff }} \quad$ Concentração efetica de dopantes $\left[\mathrm{cm}^{-3}\right]$
$\mathrm{N}_{\mathrm{AH}} \quad$ Concentração de dopantes da camada de silício do transistor GC SOI na região fortemente dopada do canal $\left[\mathrm{cm}^{-3}\right]$
$\mathrm{N}_{\mathrm{AL}} \quad$ Concentração de dopantes da camada de silício do transistor GC SOI na região fracamente dopada do canal $\left[\mathrm{cm}^{-3}\right]$
$\mathrm{N}_{\mathrm{C}} \quad$ Densidade de estados na faixa de condução
$\mathrm{N}_{\mathrm{D}} \quad$ Concentração de impurezas doadoras em um semicondutor $\left[\mathrm{cm}^{-3}\right]$
$\mathrm{N}_{\mathrm{D}}^{+} \quad$ Concentração de impurezas doadoras ionizadas em um semicondutor $\left[\mathrm{cm}^{-3}\right]$
$\mathrm{n}_{\mathrm{i}} \quad$ Concentração intrínseca de portadores $\left[\mathrm{cm}^{-3}\right]$
$\mathrm{N}_{\text {itb }} \quad$ Densidades de armadilhas de interface na segunda interface por unidade de área $\left[\mathrm{F} / \mathrm{cm}^{2}\right]$
$\mathrm{N}_{\text {iff }}$
Densidade de armadilhas de interface na primeira interface por unidade de área $\left[\mathrm{F} / \mathrm{cm}^{2}\right]$
$\mathrm{N}_{\mathrm{V}}$
Densidade de estados na faixa de valência
q
Carga elementar do elétron $\left[1,6 \times 10^{-19} \mathrm{C}\right]$
$Q_{0}$
Carga de inversão para $\mathrm{V}_{\mathrm{GF}}=\mathrm{V}_{\mathrm{thfi}}\left[\mathrm{C} / \mathrm{cm}^{2}\right]$
$Q_{D}$
Densidade de carga de inversão na fronteira do canal com a região de dreno do transistor SOI $\left[\mathrm{C} / \mathrm{cm}^{2}\right]$
Carga de depleção na camada de silício $\left[\mathrm{C} / \mathrm{cm}^{2}\right]$
$Q_{D, H D}$
Carga de depleção efetiva da camada de silício $\left[\mathrm{C} / \mathrm{cm}^{2}\right]$
Densidade de carga de inversão na fronteira do canal com a região de dreno do transistor SOI fortemente dopado $\left[\mathrm{C} / \mathrm{cm}^{2}\right]$
$Q_{D, L D}$
Densidade de carga de inversão na fronteira do canal com a região de dreno do transistor SOI fracamente dopado $\left[\mathrm{C} / \mathrm{cm}^{2}\right]$ 

$Q_{n f}$
Densidade de cargas de inversão na primeira interface do transistor SOI [C/cm $\left.{ }^{2}\right]$
$Q_{\mathrm{ox}}$
Densidade de cargas fixas no óxido de porta do transistor MOS $\left[\mathrm{C} / \mathrm{cm}^{2}\right]$
$Q_{\text {oxf }}$
Qoxb
Qs
$Q_{S B}$
$Q_{S, H D}$
$Q_{S, L D}$
$\mathrm{R}$
$r_{\mathrm{d} 2}$
$r_{d 3}$
R OUT
$S$
$\mathrm{S}_{\mathrm{NT}}$
$\mathrm{T}$
THD
$t_{\text {oxb }}$
$t_{\text {oxf }}$
$t_{\mathrm{Si}}$
$\mathrm{V}_{\mathrm{o}}$
Densidade de cargas fixas no óxido de porta na primeira interface $\left[\mathrm{C} / \mathrm{cm}^{2}\right]$
Densidade de cargas fixas no óxido enterrado na segunda interface $\left[\mathrm{C} / \mathrm{cm}^{2}\right]$
Densidade de carga de inversão na fronteira do canal com a região de fonte do transistor $\mathrm{SOI}\left[\mathrm{C} / \mathrm{cm}^{2}\right]$
Carga de acumulação $\left(Q_{S B}>0\right)$ ou inversão $\left(Q_{S B}<0\right)$ na segunda interface por unidade de área $\left[\mathrm{C} / \mathrm{cm}^{2}\right]$
Densidade de carga de inversão na fronteira do canal com a região de fonte do transistor SOI fortemente dopado $\left[\mathrm{C} / \mathrm{cm}^{2}\right]$
Densidade de carga de inversão na fronteira do canal com a região de fonte do transistor SOI fracamente dopado $\left[\mathrm{C} / \mathrm{cm}^{2}\right]$
Resistência interna da fonte de corrente utilizada para a polarização do seguidor de fonte $[\Omega]$
Resistência de saída do transistor Q2 dos espelhos de corrente (Figura 67) [ $\Omega]$
Resistência de saída do transistor Q3 dos espelhos de corrente (Figura 67) $[\Omega]$
Resistência de saída do espelho de corrente $[\Omega]$
Inclinação de sublimiar [mV/dec]
Parâmetro de ajuste que controla a transição entre os regimes de inversão fraca e forte
Temperatura absoluta $[\mathrm{K}]$
Distorção harmônica total [dB]
Espessura do óxido enterrado [nm]
Espessura do óxido de porta [nm]
Espessura da camada de silício [nm]
Componente contínua do sinal senoidal considerado na análise de distorção harmônica [V] 
Va

$V_{D}$

$\mathrm{V}_{\mathrm{D}, \mathrm{HD}}$

$V_{D, H D O}$

$V_{D, H D, L I N}$

$V_{D, H D, S A T}$

$\mathrm{V}_{\mathrm{D}, \mathrm{IN}}$

$\mathrm{V}_{\mathrm{D}, \mathrm{OUT}}$

$V_{D D}$

$V_{D E}$

$v_{d s}$

$V_{D S}$

$\mathrm{V}_{\mathrm{DS}, \mathrm{SAT}}$

$V_{D S, S A T, H D}$

$V_{D S, S A T, L D}$

$V_{\text {DSE }}$

$V_{E A}$

$\mathrm{V}_{\mathrm{FB}}$

$V_{G B}$

$\mathrm{V}_{\mathrm{GB}, \mathrm{accB}}$

$v_{g f}$

$V_{G F}$

$V_{G S}$

Amplitude do sinal senoidal aplicado à entrada de um circuito para a análise de distorção harmônica [V]

Tensão aplicada ao dreno do transistor SOI [V]

Tensão no dreno do transistor fortemente dopado [V]

Tensão no dreno do transistor fortemente dopado no instante em que o fracamente dopado atinge a saturação [V]

Tensão entre dreno e fonte do transistor fortemente dopado em região linear [V]

Tensão entre dreno e fonte do transistor fortemente dopado em saturação[V]

Tensão de entrada do espelho de corrente [V]

Tensão de saída do espelho de corrente [V]

Tensão de alimentação do amplificador [V]

Tensão de dreno efetiva [V]

Componente alternada da tensão de saída do amplificador operacional de transcondutância [V]

Tensão entre dreno e fonte [V]

Tensão de saturação [V]

Tensão de saturação do transistor fortemente dopado [V]

Tensão de saturação do transistor fracamente dopado [V]

Tensão efetiva entre dreno e fonte [V]

Tensão Early [V]

Tensão de faixa plana da estrutura MOS [V]

Tensão aplicada ao substrato do transistor SOI [V]

Tensão aplicada ao substrato para que a segunda interface acumule [V]

Componente alternada da tensão de entrada do amplificador operacional de transcondutância [V]

Tensão aplicada à primeira porta do transistor SOI [V]

Tensão entre porta e fonte do transistor MOS [V] 


\begin{tabular}{|c|c|}
\hline $\mathrm{V}_{\mathrm{GT}}$ & Sobretensão de condução [V] \\
\hline $\mathrm{V}_{\mathrm{GT}, \mathrm{HD}}$ & Sobretensão de condução do transistor fortemente dopado [V] \\
\hline $\mathrm{V}_{\mathrm{GT}, \mathrm{LD}}$ & Sobretensão de condução do transistor fracamente dopado [V] \\
\hline$V_{\mathbb{I N}}$ & Tensão de entrada do amplificador [V] \\
\hline VINTERM,SAT & $\begin{array}{l}\text { Potencial do nó intermediário do canal para a situação em que } \\
\text { os dois transistores estão saturados [V] }\end{array}$ \\
\hline$V_{\text {ip }}$ & Tensão de entrada de pico [V] \\
\hline $\mathrm{V}_{\text {OS }}$ & Excursão de saída de espelhos de corrente [V] \\
\hline $\mathrm{V}_{\text {OUT }}$ & Tensão de saída do amplificador [V] \\
\hline$V_{S}$ & Tensão aplicada à fonte do transistor SOI [V] \\
\hline $\mathrm{V}_{\mathrm{SAT}}$ & Tensão de saturação em inversão forte [V] \\
\hline$v_{\text {sat }}$ & $\begin{array}{l}\text { Velocidade de saturação dos portadores na camada de silício } \\
{[\mathrm{cm} / \mathrm{s}]}\end{array}$ \\
\hline $\mathrm{V}_{\mathrm{SAT}, \mathrm{CM}}$ & Tensão de saturação do espelho de corrente [V] \\
\hline$v_{\text {sat,e }}$ & $\begin{array}{l}\text { Velocidade de saturação dos elétrons na camada de silício } \\
\text { [cm/s] }\end{array}$ \\
\hline$v_{\text {sat, } h}$ & $\begin{array}{l}\text { Velocidade de saturação das lacunas na camada de silício } \\
{[\mathrm{cm} / \mathrm{s}]}\end{array}$ \\
\hline $\mathrm{V}_{\mathrm{sat}, \mathrm{HD}}$ & $\begin{array}{l}\text { Velocidade de saturação dos portadores na camada de silício do } \\
\text { transistor fortemente dopado [V/cm] }\end{array}$ \\
\hline$v_{\text {sat }, L D}$ & $\begin{array}{l}\text { Velocidade de saturação dos portadores na camada de silício do } \\
\text { transistor fracamente dopado }[\mathrm{V} / \mathrm{cm}]\end{array}$ \\
\hline $\mathrm{V}_{\mathrm{T}}$ & Potencial térmico $[\mathrm{V}]$ \\
\hline $\mathrm{V}_{\text {thf }}$ & Tensão de limiar da primeira interface em inversão forte [V] \\
\hline $\mathrm{V}_{\text {thfo }}$ & Tensão de limiar de um transistor de canal longo [V] \\
\hline $\mathrm{V}_{\mathrm{thf}, \mathrm{HD}}$ & $\begin{array}{l}\text { Tensão de limiar da primeira interface da região fortemente } \\
\text { dopada do transistor GC SOI em inversão forte [V] }\end{array}$ \\
\hline$V_{\mathrm{thf}, L \mathrm{D}}$ & $\begin{array}{l}\text { Tensão de limiar da primeira interface da região fracamente } \\
\text { dopada do transistor GC SOI em inversão forte [V] }\end{array}$ \\
\hline $\mathrm{V}_{\text {thf,accB }}$ & $\begin{array}{l}\text { Tensão de limiar da primeira interface com a segunda interface } \\
\text { acumulada [V] }\end{array}$ \\
\hline
\end{tabular}




\begin{tabular}{|c|c|}
\hline$V_{\text {thf,deplB }}$ & $\begin{array}{l}\text { Tensão de limiar da primeira interface com a segunda interface } \\
\text { depletada [V] }\end{array}$ \\
\hline $\mathrm{V}_{\mathrm{thf}, \mathrm{invB}}$ & $\begin{array}{l}\text { Tensão de limiar da primeira interface com a segunda interface } \\
\text { invertida [V] }\end{array}$ \\
\hline $\mathrm{V}_{\text {thfi }}$ & $\begin{array}{l}\text { Tensão de limiar equivalente da primeira interface do transistor } \\
\text { SOI em inversão fraca [V] }\end{array}$ \\
\hline$V_{\text {thfi,HD }}$ & $\begin{array}{l}\text { Tensão de limiar equivalente da primeira interface da região } \\
\text { fortemente dopada do transistor GC SOI em inversão fraca [V] }\end{array}$ \\
\hline$V_{\text {thfi,LD }}$ & $\begin{array}{l}\text { Tensão de limiar equivalente da primeira interface da região } \\
\text { fracamente dopada do transistor GC SOI em inversão fraca [V] }\end{array}$ \\
\hline $\mathrm{V}_{\text {thf,mean }}$ & Tensão de limiar média [V] \\
\hline W & Largura do canal do transistor $[\mu \mathrm{m}]$ \\
\hline $\mathrm{x}$ & Eixo na direção da profundidade da camada de silício \\
\hline$X_{\text {dmax }}$ & Profundidade máxima da região de depleção $[\mu \mathrm{m}]$ \\
\hline $\mathrm{y}$ & Eixo na direção do comprimento de canal do transistor SOI \\
\hline$\alpha$ & $\begin{array}{l}\text { Parâmetro resultante da associação das capacitâncias do } \\
\text { transistor MOS }\end{array}$ \\
\hline$\alpha_{M}$ & $\begin{array}{l}\text { Parâmetro de ajuste para o cálculo da corrente gerada por } \\
\text { ionização por impacto }\left[\mathrm{m}^{-1}\right]\end{array}$ \\
\hline$\alpha_{s}$ & Constante de espalhamento $[\mathrm{cm} / \mathrm{V}]$ \\
\hline$\alpha_{V_{T}}$ & $\begin{array}{l}\text { Parâmetro utilizado no cálculo da variação da tensão de limiar } \\
\text { com a temperatura }\end{array}$ \\
\hline$\beta$ & Fator de corrente \\
\hline$\beta_{\mathrm{M}}$ & $\begin{array}{l}\text { Parâmetro de ajuste para o cálculo da corrente gerada por } \\
\text { ionização por impacto }[\mathrm{V} / \mathrm{m}]\end{array}$ \\
\hline$\beta_{\text {mean }}$ & Valor médio do fator de corrente \\
\hline$\varepsilon_{0 x}$ & Permissividade do óxido de silício $\left[3,45 \times 10^{-13} \mathrm{~F} / \mathrm{cm}\right]$ \\
\hline$\varepsilon_{\mathrm{Si}}$ & Permissividade do silício $\left[1,06 \times 10^{-12} \mathrm{~F} / \mathrm{cm}\right]$ \\
\hline$\Delta \mathrm{L}$ & $\begin{array}{l}\text { Redução do comprimento efetivo de canal devido à difusão } \\
\text { lateral }[\mu \mathrm{m}]\end{array}$ \\
\hline$\Delta \mathrm{I}_{D S} / \mathrm{l}_{\mathrm{DS}}$ & Descasamento na corrente de dreno \\
\hline
\end{tabular}


$\Delta \mathrm{k}_{\mathrm{Q}} / \mathrm{k}_{\mathrm{Q}}$

$\Delta \mathrm{V}_{\text {thf }} / \mathrm{V}_{\mathrm{thf}}$

$\Delta \beta / \beta$

$\phi_{\mathrm{F}}$

$\phi_{\mathrm{MS}}$

$\phi_{M S B}$

$\phi_{\mathrm{MSF}}$

$\phi_{S B}$

$\phi_{\text {SF }}$

$\lambda$

$\mu_{0}$

$\mu_{\mathrm{CC}}$

$\mu_{\mathrm{ii}}$

$\mu_{n}$

$\mu_{\mathrm{nHD}}$

$\mu_{\mathrm{ni}}$

$\mu_{\mathrm{nLD}}$

$\mu_{\mathrm{pe}}$

$\mu_{\mathrm{ph}}$
Descasamento no termo dependente das cargas na corrente de dreno

Descasamento na tensão de limiar

Descasamento no fator de corrente

Potencial de Fermi [V]

Diferença da função de trabalho entre metal e silício [V]

Diferença de função de trabalho entre o substrato e a camada de silício [V]

Diferença da função de trabalho entre metal de porta e semicondutor [V]

Potencial de superfície da segunda interface do transistor SOI [V]

Potencial de superfície da primeira interface do transistor SOI [V]

Parâmetro que considera o efeito de modulação do comprimento de canal devido à tensão aplicada ao dreno [ $\left.\mathrm{V}^{-1}\right]$

Mobilidade dos portadores independente de campo elétrico $\left[\mathrm{cm}^{2} / \mathrm{V} . \mathrm{s}\right]$

Mobilidade dos portadores lacunas devido ao espalhamento portador-portador [ $\left.\mathrm{cm}^{2} / \mathrm{V} . \mathrm{s}\right]$

Mobilidade dos portadores devido ao espalhamento por impurezas ionizadas [ $\left.\mathrm{cm}^{2} / \mathrm{V} . \mathrm{s}\right]$

Mobilidade efetiva dos elétrons na região do canal $\left[\mathrm{cm}^{2} N\right.$.s]

Mobilidade efetiva dos elétrons na região fortemente dopada do canal do transistor GC SOI [ $\mathrm{cm}^{2} N$.s]

Mobilidade dos portadores devido ao espalhamentos por impurezas neutras [ $\left.\mathrm{cm}^{2} / \mathrm{V} . \mathrm{s}\right]$

Mobilidade efetiva dos elétrons na região fracamente dopada do canal do transistor GC SOI [ $\mathrm{cm}^{2} N$.s]

Mobilidade dos elétrons devido ao espalhamento de rede $\left[\mathrm{cm}^{2} /\right.$ V.s]

Mobilidade das lacunas devido ao espalhamento de rede $\left[\mathrm{cm}^{2} /\right.$ V.s] 


\begin{tabular}{|c|c|}
\hline$\mu_{\mathrm{ps}}$ & $\begin{array}{l}\text { Mobilidade dos portadores devido ao espalhamento de rede } \\
{\left[\mathrm{cm}^{2} / \text { V.s] }\right.}\end{array}$ \\
\hline$\mu_{\mathrm{psii}}$ & $\begin{array}{l}\text { Mobilidade dos portadores devido aos espalhamentos de rede e } \\
\text { por impurezas ionizadas }\left[\mathrm{cm}^{2} / \text { V.s] }\right.\end{array}$ \\
\hline$\mu_{\mathrm{psiih}}$ & $\begin{array}{l}\text { Mobilidade das lacunas devido aos espalhamentos de rede e por } \\
\text { impurezas ionizadas }\left[\mathrm{cm}^{2} / \text { V.s] }\right.\end{array}$ \\
\hline$\mu_{\mathrm{psiie}}$ & $\begin{array}{l}\text { Mobilidade dos elétrons devido aos espalhamentos de rede e } \\
\text { por impurezas ionizadas }\left[\mathrm{cm}^{2} / \mathrm{V} . \mathrm{s}\right]\end{array}$ \\
\hline$\sigma$ & Parâmetro que considera o efeito de DIBL \\
\hline$\sigma l_{D S}$ & Desvio padrão da corrente \\
\hline$\sigma \mathrm{k}_{\mathrm{Q}}$ & $\begin{array}{l}\text { Desvio padrão do termo dependente das cargas na corrente de } \\
\text { dreno }\end{array}$ \\
\hline$\sigma\left(\mathrm{N}_{\mathrm{A}}^{-} / \mathrm{N}_{\mathrm{A}}\right)$ & Desvio padrão da porcentagem de impurezas ionizadas \\
\hline$\sigma_{\mathrm{V}_{\text {thf }}}$ & Desvio padrão da tensão de limiar \\
\hline$\sigma_{0 V_{\text {thf }}}$ & $\begin{array}{l}\text { Desvio padrão da tensão de limiar normalizado em relação à } \\
\text { área de porta }\end{array}$ \\
\hline$\sigma_{\bigvee_{\mathrm{thf}, \mathrm{CED}}}$ & $\begin{array}{l}\text { Desvio padrão da tensão de limiar devido à variação no número } \\
\text { de dopantes na camada de depleção }\end{array}$ \\
\hline$\sigma \mathrm{V}_{\mathrm{DS}, \mathrm{SAT}}$ & Desvio padrão da tensão de saturação \\
\hline$\sigma \beta$ & Desvio padrão do fator de corrente \\
\hline
\end{tabular}




\section{SUMÁRIO}

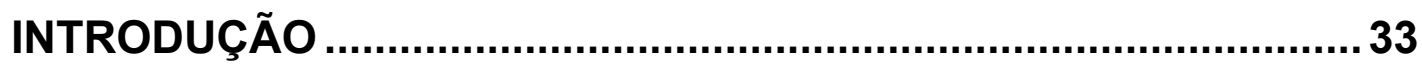

2 CONCEITOS BÁSICOS................................................................... 37

$2.1 \quad$ TECNOLOGIA SILÍCIO-SOBRE-ISOLANTE ..............................................

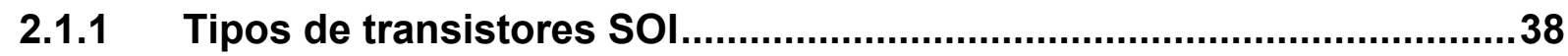

2.1.2 Vantagens do transistor SOI totalmente depletado ..............................41

2.1.3 Transistores com canal tensionado ....................................................42

2.2 INFLUÊNCIA DA REDUÇÃO DA TEMPERATURA NAS PROPRIEDADES ELÉTRICAS DOS SEMICONDUTORES ….............................................................

2.2.1 Concentração intrínseca de portadores e largura da faixa proibida ....44

2.2.2 Ionização incompleta de portadores e Potencial de Fermi ....................46

2.3 PARÂMETROS ELÉTRICOS DO TRANSISTOR SOI CONVENCIONAL CONSIDERANDO A INFLUÊNCIA DA REDUÇÃO DA TEMPERATURA.................47

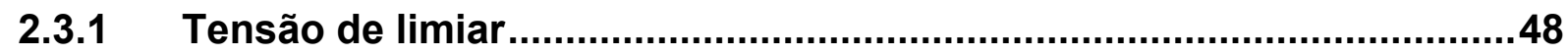

2.3.1.1 Variação da tensão de limiar com a temperatura ..................................49

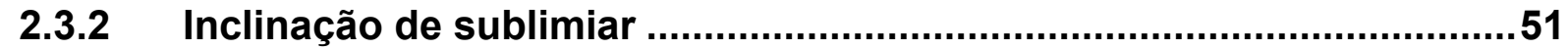

2.3.3 Efeitos de canal curto ..........................................................................

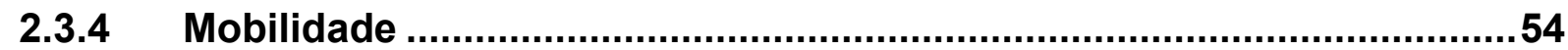

2.3.4.1 Velocidade de saturação do portador .................................................59

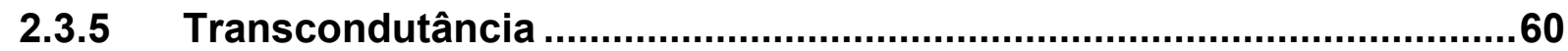

2.3.6 lonização por impacto …......................................................................61

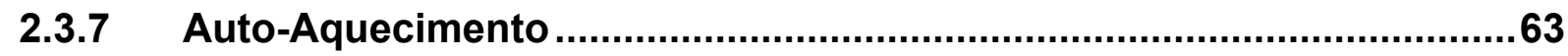

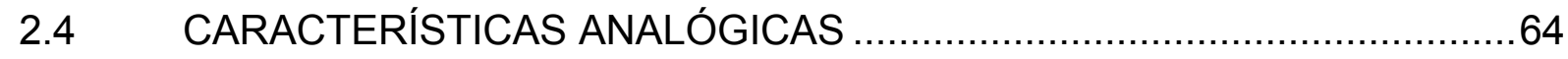

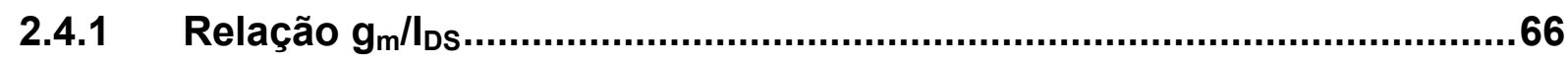

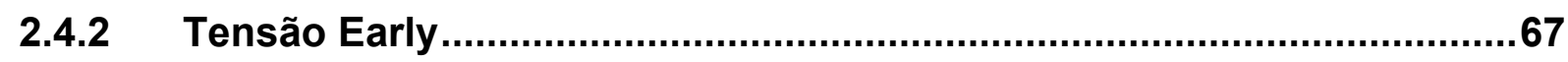




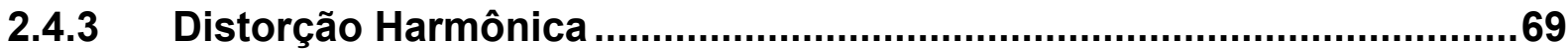

2.5 TRANSISTORES SOI DE CANAL GRADUAL ……............................70

2.5.1 Modelo analítico contínuo para transistores GC SOI ...........................73

2.6 SIMULAÇÃO NUMÉRICA BIDIMENSIONAL EM FUNÇÃO DA

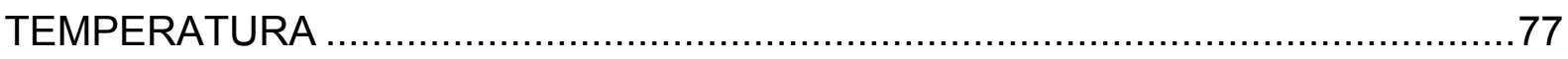

3 MODELAGEM DA CORRENTE DE DRENO DE TRANSISTORES

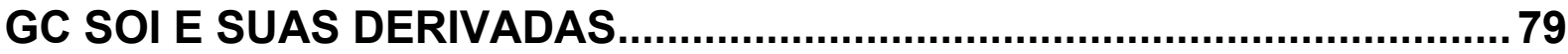

3.1 ADEQUAÇÃO DO MODELO ANALÍTICO CONTÍNUO PARA OPERAÇÃO EM BAIXAS TEMPERATURAS .................................................................... 79

3.1.1 Validação do modelo através da comparação com dados experimentais 80

3.2 INCLUSÃO DO MODELO ANALÍTICO EM UM SIMULADOR DE CIRCUITOS. 86

3.2.1 Validação da implementação através da comparação com dados simulados 87

3.3 EQUAÇÕES ANALÍTICAS PARA TRANSCONDUTÂNCIA E CONDUTÂNCIA DE DRENO DE TRANSISTORES GC SOI ..................................93

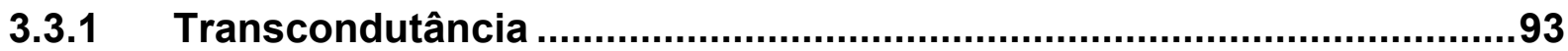

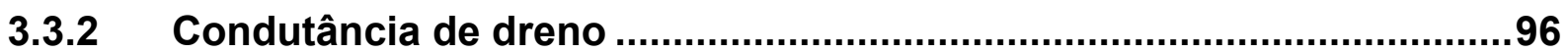

3.3.3 Comparação com resultados experimentais ........................................97

4 ESTUDO DE APLICAÇÕES ANALÓGICAS DE TRANSISTORES GC SOI EM BAIXAS TEMPERATURAS …............................................ 102

4.1 FABRICAÇÃO DE CÉLULAS ANALÓGICAS SIMPLES .........................102

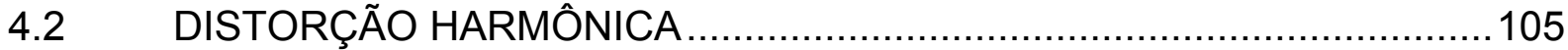

4.2.1 Análise da distorção harmônica em temperatura ambiente ................105

4.2.2 Análise da distorção harmônica em baixas temperaturas ...................110

4.3 ANÁLISE DE DESCASAMENTO EM TRANSISTORES GC SOI .............114

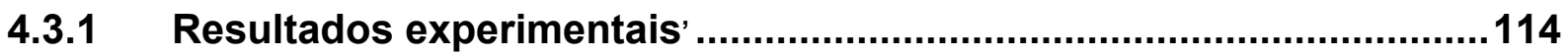


4.3.2 Análise do descasamento utilizando o modelo analítico contínuo ....119

4.3.3 Resultados das simulações numéricas bidimensionais .123

4.3.4 Análise experimental do descasamento em função da temperatura .126 4.4 UTILIZAÇÃO DE TRANSISTORES GC SOI COMO AMPLIFICADOR DRENO COMUM 131

4.4.1 Análise experimental em temperatura ambiente ' .............................. 132

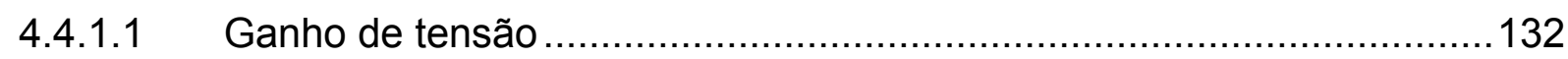

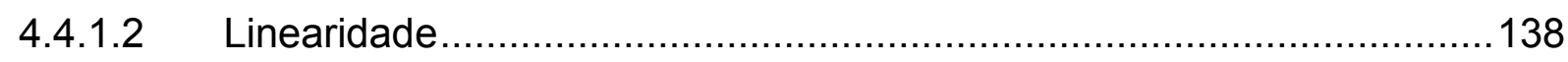

4.4.2 Influência da ionização por impacto no ganho de tensão de seguidores de fonte

4.4.3 Influência da redução do comprimento de canal sobre o desempenho de seguidores de fonte 143

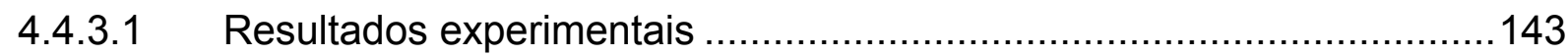

4.4.3.2 Simulações numéricas bidimensionais ..................................... 147

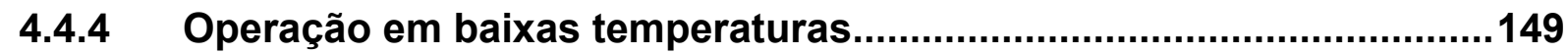

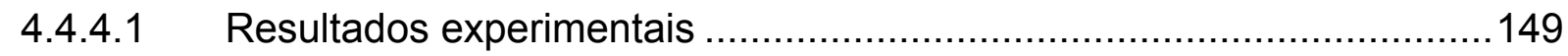

4.4.4.2 Simulações numéricas bidimensionais ......................................151

4.5 ESPELHOS DE CORRENTE IMPLEMENTADOS COM TRANSISTORES GC SOI OPERANDO EM BAIXAS TEMPERATURAS ................................. 155

$5 \quad$ TRANSISTORES SOI COM CANAL TENSIONADO ..................... 162

5.1 CARACTERÍSTICAS DOS DISPOSITIVOS CARACTERIZADOS ...........162

5.2 OPERAÇÃO DE TRANSISTORES COM TENSÃO UNIAXIAL EM BAIXAS

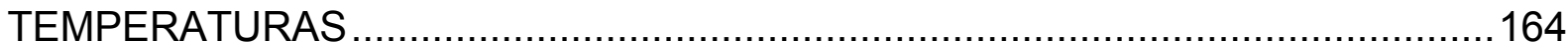

5.2.1 Ganho de transcondutância e inclinação de sublimiar .......................164

5.2.2 Características Analógicas de Transistores com Tensão Uniaxial ....168

5.3 COMPARAÇÃO ENTRE TRANSISTORES COM TENSÃO MECÂNICA UNIAXIAL E BIAXIAL EM BAIXAS TEMPERATURAS 171 


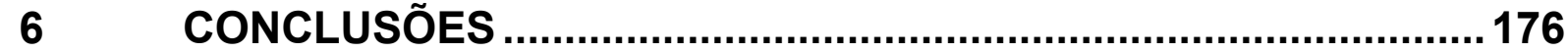

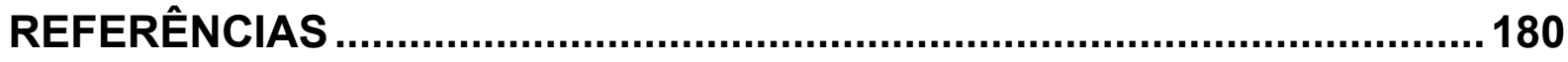

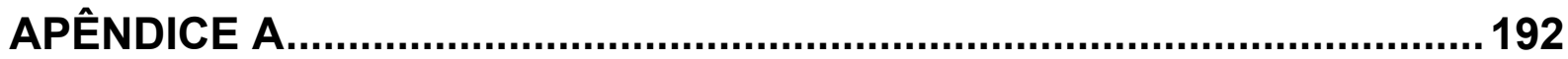

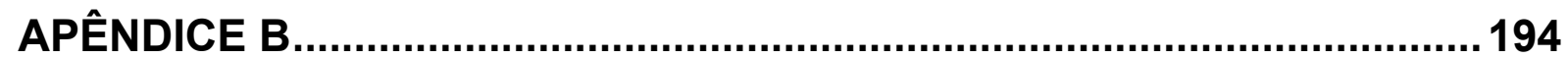

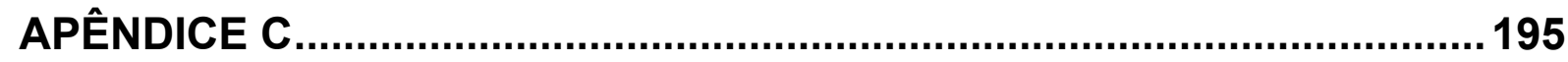




\section{INTRODUÇÃO}

A tecnologia de fabricação de circuitos integrados em lâminas de silício sobre isolante (Silicon-On-Insulator - SOI) surgiu como uma alternativa para a tecnologia CMOS convencional, na fabricação de circuitos integrados em altíssima escala de integração (Ultra Large Scale Integration - ULSI) ${ }^{1}$. Nesta tecnologia, os dispositivos são fabricados em uma fina camada de silício, separada do restante do substrato por um material isolante. A isolação dielétrica intrínseca entre os dispositivos e o substrato, proporcionada pela presença da camada isolante, permite minimizar, ou em alguns casos, até mesmo suprimir, efeitos parasitários decorrentes da redução das dimensões da tecnologia MOS convencional. Em seus primórdios, a tecnologia SOI permaneceu restrita a poucas aplicações, como operação em ambientes sujeitos à radiação ${ }^{2}$ e altas temperaturas ${ }^{3}$, devido à baixa qualidade das lâminas SOI disponíveis ${ }^{1,4}$. Entretanto, a partir da década de 80 , esta tecnologia começou a emergir como uma real alternativa para a tecnologia MOS convencional, devido à enorme quantidade de estudos efetuados nas áreas de materiais e dispositivos SOI, permitindo a obtenção de lâminas de boa qualidade e menor custo, possibilitando sua utilização em um maior número de aplicações ${ }^{1}$.

A redução da espessura da camada de silício sobre o isolante deu origem aos dispositivos SOI de camada fina que, quando operam com a região do canal totalmente depletada, apresentam vantagens adicionais, tais como aumento da mobilidade dos portadores na camada de inversão ${ }^{5}$ e da transcondutância, redução dos efeitos de canal curto ${ }^{6}$, do campo elétrico transversal, entre outros benefícios 7,8,9,10. Apesar do grande número de vantagens, os transistores SOI apresentam reduzida tensão de ruptura de dreno, devido ao efeito de corpo flutuante, que leva à ativação do transistor bipolar parasitário associado ao transistor $\mathrm{MOS}^{11}$.

Com o intuito de reduzir a ocorrência dos efeitos bipolares parasitários, e assim aumentar a tensão de ruptura dos dispositivos SOI, foi projetada uma nova estrutura denominada transistor SOI de Canal Gradual (Graded-Channel (GC) SOI MOSFET) ${ }^{12}$. Esta estrutura apresenta um perfil de concentração de dopantes assimétrico na região do canal do transistor, resultante de uma pequena modificação no processo de fabricação dos transistores SOI convencionais. Para a obtenção do 
transistor com canal gradual, uma região próxima ao dreno do transistor nMOS é protegida durante a etapa de implantação iônica para o ajuste da tensão de limiar, preservando-a com a dopagem natural da lâmina.

Além de minimizar a ocorrência de efeitos bipolares parasitários, a estrutura GC SOI MOSFET promove uma melhora em suas características de saída em relação à estrutura uniformemente dopada, tais como aumento da transcondutância e redução da condutância de dreno, que implica em maior tensão Early. Estas características são extremamente interessantes para transistores MOS utilizados principalmente em circuitos analógicos para aplicações com baixa tensão de alimentação e baixo consumo de potência (as chamadas aplicações low-power lowvoltage - LPLV $)^{13}$. Estudos realizados em blocos analógicos básicos em temperatura ambiente, tais como espelhos de corrente e amplificadores operacionais ${ }^{13,14}$ demonstram o enorme potencial dos dispositivos GC SOI para aplicações analógicas. Outros trabalhos reportam ainda vantagens da estrutura GC SOI para aplicações em microondas ${ }^{15}$ e rádio-freqüência ${ }^{16}$.

A operação de transistores MOS em baixas temperaturas oferece importantes melhorias em seu desempenho quando comparado à operação em temperatura ambiente, tais como redução na inclinação de sublimiar e aumento na mobilidade dos portadores, entre outros, sem a necessidade de escalamento das dimensões ${ }^{17}$. Tradicionalmente, estas melhorias são exploradas com vistas a aplicações digitais e poucos trabalhos disponíveis na literatura relatam as potenciais melhoras obtidas em circuitos analógicos. Entretanto, circuitos analógicos com alto desempenho operando em ambientes criogênicos são de grande importância, por exemplo, para a eletrônica aeroespacial $^{18}$.

Este trabalho tem como principal objetivo o estudo de aplicações analógicas de transistores GC SOI e SOI convencionais, em função da temperatura, desde $380 \mathrm{~K}$ até $90 \mathrm{~K}$, combinando as vantagens oriundas da redução da temperatura com aquelas propiciadas pela estrutura SOI de canal gradual. A adequação de modelos analíticos para permitir o projeto de circuitos em baixas temperaturas e sua incorporação em simuladores de circuitos também são apresentadas, bem como um estudo comparativo das propriedades de distorção harmônica (ou linearidade), o descasamento de alguns parâmetros elétricos, tais como a tensão de limiar e a corrente de dreno, e a aplicação destes transistores em blocos analógicos básicos.

Adicionalmente, apresentamos um estudo experimental sobre transistores 
SOI nMOSFET totalmente depletados com tensão mecânica agindo sobre o canal, operando em baixas temperaturas. Essa estrutura vem sendo considerada como uma alternativa tecnológica para o problema de degradação da mobilidade observado devido ao escalamento das dimensões dos transistores MOS, uma vez que é capaz de proporcionar o aumento da mobilidade dos transistores e, conseqüentemente de sua corrente de dreno ${ }^{19,20}$.

Este trabalho está dividido em 6 capítulos, os quais estão organizados conforme descrito:

No Capítulo 2 apresentamos os fundamentos teóricos que sustentam o desenvolvimento do trabalho, discutindo os tipos de transistores SOI convencionais e suas principais características elétricas e parâmetros analógicos, bem como o efeito da temperatura sobre estes. É também apresentado o transistor SOI de canal gradual, abordando suas principais vantagens em relação ao SOI convencional, e o modelo analítico existente para esta estrutura.

O Capítulo 3 aborda a modelagem da corrente de dreno para transistores de canal gradual, descrevendo as alterações necessárias para a inclusão dos efeitos decorrentes da redução da temperatura. A implementação deste modelo em um simulador de circuitos e sua validação através da comparação com dados experimentais e oriundos de simulações numéricas são também apresentadas. Este capítulo apresenta ainda o desenvolvimento de equações analíticas contínuas para a transcondutância e condutância de dreno de transistores GC SOI, visando sua utilização como ferramenta de projeto de células analógicas.

Um estudo de características e aplicações analógicas dos transistores GC SOI em baixas temperaturas é apresentado no Capítulo 4. A linearidade e o descasamento da tensão de limiar e corrente de dreno, parâmetros importantes do ponto de vista analógico, são analisados, utilizando resultados experimentais e provenientes do modelo analítico proposto. São ainda apresentadas as vantagens dos transistores de canal gradual quando utilizados em espelhos de corrente e como amplificador dreno comum (seguidores de fonte).

O Capítulo 5 apresenta resultados experimentais de transistores SOI com tensão mecânica uniaxial e biaxial agindo sobre o canal, explicitando as vantagens de sua utilização em baixas temperaturas, principalmente no que diz respeito aos parâmetros analógicos. 
Finalmente, o Capítulo 6 apresenta as principais conclusões deste trabalho, assim como sugestões para a continuidade do que foi até então desenvolvido. 


\section{CONCEITOS BÁSICOS}

Neste capítulo será apresentada uma revisão bibliográfica sobre a tecnologia Silício sobre Isolante $(\mathrm{SOI})$, os tipos de transistores $\mathrm{SOI}$, transistores $\mathrm{SOI}$ convencionais e de canal gradual (Graded-Channel (GC) SOI nMOSFETs), enfatizando as características analógicas e modelos analíticos para a simulação destes dispositivos. Alguns efeitos decorrentes da redução da temperatura sobre o funcionamento de dispositivos semicondutores, com especial ênfase aos transistores SOI, também serão apresentados, bem como modelos analíticos que os descrevem.

\subsection{TECNOLOGIA SILÍCIO-SOBRE-ISOLANTE}

$\mathrm{Na}$ tecnologia MOS convencional (bulk MOS), os transistores são fabricados em lâminas de silício com espessuras de centenas de micrometros, embora apenas o primeiro micrometro, ou uma fração dele, em sua superfície, seja efetivamente utilizado para a fabricação dos dispositivos. A interação entre a região ativa e o restante do substrato dá origem a uma série de efeitos indesejáveis, tais como o efeito tiristor parasitário, inerente à estrutura CMOS, e elevadas capacitâncias parasitárias. Com a miniaturização dos transistores, estes efeitos tornam-se mais pronunciados ${ }^{1}$, resultando na necessidade de complexas alterações e adições de etapas de fabricação para minimizar ou solucionar estes efeitos indesejáveis, tais como a implantação de halo ${ }^{21}$ e isolação por trincheira (STI - Shallow Trench Isolation $)^{22}$.

O conceito básico da tecnologia SOI consiste na separação da região ativa da lâmina, onde os dispositivos são fabricados, do restante do substrato, por meio de uma camada de isolante. $O$ isolamento dielétrico intrínseco resultante da presença da camada de isolante (óxido enterrado) proporciona a redução de alguns dos efeitos parasitários existentes na tecnologia MOS convencional, tais como as capacitâncias de junção entre fonte/dreno e substrato, e a supressão de outros, como, por exemplo, o efeito tiristor parasitário ${ }^{23}$. Além disso, a ausência de 
cavidades nesta tecnologia simplifica enormemente o processo de fabricação dos transistores em comparação à tecnologia MOS convencional ${ }^{1}$.

A Figura 1 apresenta o perfil transversal de um transistor SOI nMOSFET, indicando os eletrodos de porta $\left(\mathrm{V}_{\mathrm{GF}}\right)$, substrato $\left(\mathrm{V}_{\mathrm{GB}}\right)$, fonte $\left(\mathrm{V}_{\mathrm{S}}\right)$ e dreno $\left(\mathrm{V}_{\mathrm{D}}\right)$, as espessuras dos óxidos de porta ( $t_{\text {oxf }}$ ) e enterrado $\left(t_{o x b}\right)$ e da camada de silício $\left(t_{S i}\right)$, bem como as três interfaces $\mathrm{Si}-\mathrm{SiO}_{2}$ existentes na estrutura.

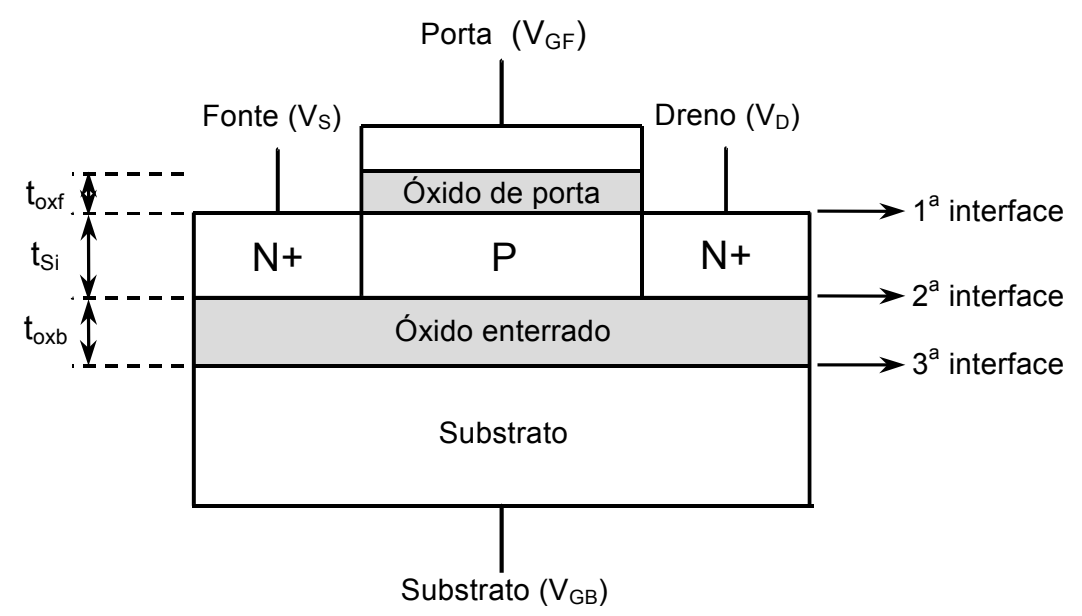

Figura 1 - Perfil transversal de um transistor SOI nMOSFET.

\subsubsection{Tipos de transistores SOI}

De acordo com o modo de funcionamento, os dispositivos SOI MOSFET podem ser classificados em modo enriquecimento (ou inversão) ou modo acumulação. No caso de transistores SOI de canal tipo N, o modo enriquecimento é o mais comum ${ }^{1}$ e, por esta razão, somente este será considerado neste trabalho.

Em um dispositivo MOS convencional, a região de depleção estende-se, a partir da interface $\mathrm{Si}-\mathrm{SiO}_{2}$, até a profundidade máxima de depleção, $\mathbf{x}_{\mathrm{dmax}}$, dada $\operatorname{por}^{24}$ :

$$
\mathrm{X}_{\mathrm{dmax}}=\sqrt{\frac{2 \varepsilon_{\mathrm{Si}} \cdot 2 \phi_{\mathrm{F}}}{\mathrm{q} \cdot \mathrm{N}_{\mathrm{A}}}}
$$

onde $\varepsilon_{S i}$ é a permissividade do silício, q é a carga elementar do elétron, $N_{A}$ é a concentração de impurezas aceitadoras do substrato e $\phi_{F}$ é o potencial de Fermi, 
dado por $\phi_{\mathrm{F}}=\frac{\mathrm{kT}}{\mathrm{q}} \cdot \ln \left(\frac{\mathrm{N}_{\mathrm{A}}}{\mathrm{n}_{\mathrm{i}}}\right)$, onde $\mathrm{k}$ é a constante de Boltzmann, T é a temperatura absoluta e $n_{i}$ é a concentração intrínseca de portadores.

No caso de transistores SOI, as características físicas são fortemente dependentes da espessura e concentração de dopantes da camada de silício sobre a qual são fabricados, bem como da temperatura de operação. Em função da combinação destes parâmetros, três tipos de transistores podem ser obtidos: dispositivos de camada fina, de camada média e de camada espessa.

Se a espessura da camada de silício, $t_{s i}$, for maior que 2.x $x_{d m a x}$, não haverá interação entre as regiões de depleção induzidas a partir da primeira e segunda interfaces, dando origem a uma região neutra entre elas. Neste caso, o dispositivo é chamado de transistor SOI de camada espessa ou parcialmente depletado (partially depleted - PD). A região neutra existente nos dispositivos PD SOI pode possuir um contato elétrico ou não. Caso exista o contato de corpo e este estiver aterrado, o dispositivo terá comportamento semelhante ao de um MOSFET convencional. Caso este contato seja deixado eletricamente flutuando, o transistor apresentará efeitos de corpo flutuante ${ }^{25}$, tais como o efeito de elevação abrupta de corrente (Efeito Kink) e o efeito bipolar parasitário entre fonte e dreno ${ }^{1}$. As primeiras aplicações industriais da tecnologia SOI utilizaram a opção de camada espessa ${ }^{26,27,28}$, justamente pela similaridade de operação em relação aos MOSFETs convencionais, combinada com a já mencionada redução das capacitâncias de junção ${ }^{29}$.

Caso a espessura da camada de silício seja menor que a profundidade máxima de depleção do dispositivo, ou seja, $t_{\mathrm{S}_{i}}<\mathrm{x}_{\mathrm{dmax}}$, as regiões de depleção da primeira e segunda interfaces entrarão em contato e a camada de silício certamente estará totalmente depletada para tensões de porta superiores à tensão de limiar, independentemente da tensão aplicada ao substrato (com exceção da possível presença de uma fina camada de acumulação ou inversão na segunda interface, se uma alta tensão negativa ou positiva for aplicada ao substrato, respectivamente). Esta interação entre as regiões de depleção faz com que os potenciais de superfície da primeira e segunda interfaces ( $\phi_{S F}$ e $\phi_{S B}$, respectivamente) estejam interrelacionados, promovendo o acoplamento elétrico da estrutura.

Neste caso, o dispositivo é chamado de transistor SOl de camada fina e totalmente depletado (fully depleted - FD). Estes dispositivos apresentam as 
características elétricas mais atrativas entre os transistores SOI, tais como redução do campo elétrico horizontal ${ }^{30}$, maior mobilidade dos portadores na região do canal $^{31}$, menor variação da tensão de limiar com a temperatura ${ }^{32}$, menor ocorrência de efeitos de canal curto ${ }^{6}$, entre outras. Em virtude destas vantagens, somente estes transistores serão utilizados no decorrer deste trabalho. Vale ressaltar que dispositivos FD SOI apresentam dependência da tensão de limiar com a espessura da camada de silício, que é indesejável na operação de circuitos ${ }^{33}$.

Finalmente, os dispositivos de camada média (near-fully depleted SOI - NFD SOI) são aqueles em que $x_{d \max }<t_{S i}<2$. $x_{d \max }$. Neste caso, as regiões de depleção da primeira e segunda interfaces poderão ou não entrar em contato, dependendo da tensão aplicada ao substrato $\left(\mathrm{V}_{\mathrm{GB}}\right)$ do dispositivo. Desta forma, os dispositivos de camada média podem apresentar comportamento elétrico de um SOI parcialmente ou totalmente depletado.

A Figura 2 apresenta os diagramas de faixas de energia dos transistores MOS convencional (A), SOI de camada espessa (B) e SOI de camada fina (C). Nesta figura, $E_{C}$ representa o nível energético inferior da faixa de condução, $E_{V}$ o nível energético superior da faixa de valência, $E_{i}$ o nível intrínseco, $E_{F}$ o nível de Fermi da camada de semicondutor (silício, no caso da tecnologia SOI), $\mathrm{E}_{\mathrm{FM}}$ o nível de Fermi do eletrodo de porta e $\mathrm{E}_{\mathrm{FB}}$ o nível de Fermi do substrato.

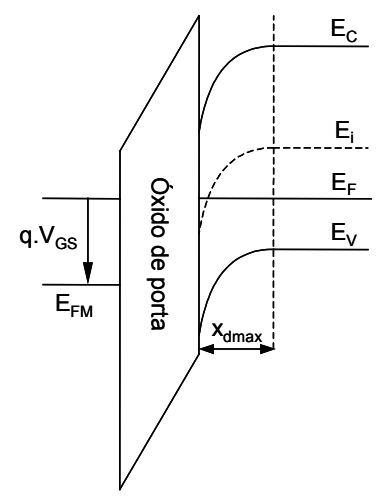

(A)

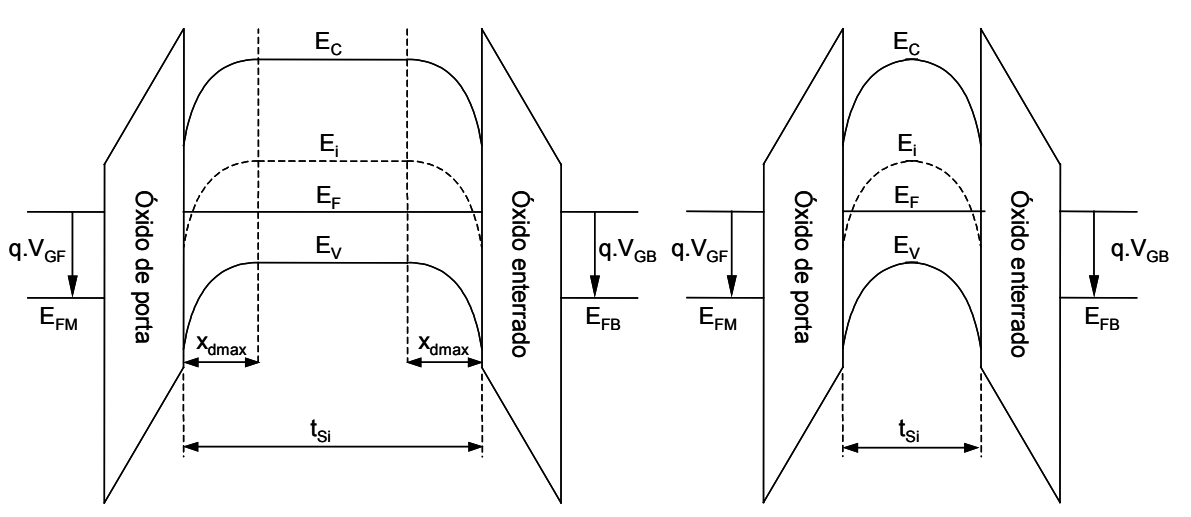

(B)

(C)

Figura 2 - Diagramas de faixas de energia para transistores MOS convencional $(A)$, SOI de camada espessa (B) e SOI de camada fina (C). 


\subsubsection{Vantagens do transistor SOI totalmente depletado}

Grande parte das vantagens apresentadas pelos transistores SOI MOSFET totalmente depletados sobre o MOSFET convencional e o SOI MOSFET parcialmente depletado está associada ao fator de corpo (n), que influencia diretamente a capacidade de fornecimento de corrente do dispositivo ${ }^{34}$.

O fator de corpo pode ser entendido como a oposição que o dispositivo oferece para que o eletrodo de porta controle o potencial de superfície da primeira interface e é dado por:

$$
\mathrm{n}=1+\alpha
$$

onde $\alpha$ é o fator de acoplamento capacitivo do transistor MOS, dado por:

$\alpha=\frac{C_{D}}{C_{o x}}$ para transistores MOS convencionais e SOI parcialmente depletados;

$\alpha=\frac{\mathrm{C}_{\mathrm{Si}}}{\mathrm{C}_{\mathrm{oxf}}}$ para transistores FD SOI com a segunda interface acumulada;

$\alpha=\frac{\mathrm{C}_{\mathrm{Si}} \mathrm{C}_{\text {oxb }}}{\mathrm{C}_{\mathrm{oxf}}\left(\mathrm{C}_{\mathrm{Si}}+\mathrm{C}_{\mathrm{oxb}}\right)}$ para transistores FD SOI com a segunda interface depletada;

Nas expressões acima, $C_{D}, C_{o x f}\left(C_{o x}\right.$ para os transistores MOS convencionais), $\mathrm{C}_{\mathrm{oxb}}$ e $\mathrm{C}_{\mathrm{Si}}$ são as capacitâncias da região de depleção, do óxido de porta, do óxido enterrado e da camada de silício, respectivamente, todas por unidade de área e expressas por $C_{D}=\frac{\varepsilon_{S i}}{x_{d \max }} ; C_{o x f}=\frac{\varepsilon_{o x}}{t_{o x f}} ; C_{o x b}=\frac{\varepsilon_{o x}}{t_{o x b}}$ e $C_{S i}=\frac{\varepsilon_{S i}}{t_{S i}}$, onde $\varepsilon_{0 \mathrm{x}}$ é a permissividade do óxido.

Verifica-se que o valor de $\mathrm{n}$ é menor nos dispositivos SOI totalmente depletados que nos dispositivos MOS convencionais ${ }^{35}$, se aproximando da unidade. Os valores numéricos de $\mathrm{n}$ seguem a seguinte ordem:

$$
\mathrm{n} \text { sOI totalmente depletado }<\mathrm{n} \text { MOS convencional }<\mathrm{n} \text { sol com a segunda interface acumulada }
$$

Devido ao menor fator de corpo nos transistores SOI totalmente depletados, estes apresentam maior intensidade de corrente quando comparados a dispositivos 
convencionais com tensões de limiar idênticas e polarizados com mesma tensão ${ }^{1}$. Tal fato pode ser observado a partir das equações da corrente de dreno simplificadas, nas regiões $\mathrm{de}^{36}$ :

- triodo:

$$
\mathrm{I}_{\mathrm{DS}} \cong \frac{\mathrm{W} \mu_{\mathrm{n}} \mathrm{C}_{\mathrm{oxf}}}{\mathrm{L}}\left[\left(\mathrm{V}_{\mathrm{GF}}-\mathrm{V}_{\mathrm{thf}}\right) \mathrm{V}_{\mathrm{DS}}-\mathrm{n} \frac{\mathrm{V}_{\mathrm{DS}}^{2}}{2}\right]
$$

- saturação:

$$
\mathrm{I}_{\mathrm{DSsat}} \cong \frac{\mathrm{W} \mu_{\mathrm{n}} \mathrm{C}_{\mathrm{oxf}}}{2 \mathrm{Ln}}\left(\mathrm{V}_{\mathrm{GF}}-\mathrm{V}_{\mathrm{thf}}\right)^{2}
$$

onde W e L são, respectivamente, a largura e o comprimento de canal do transistor, $\mu_{n}$ é a mobilidade efetiva dos elétrons no canal e $V_{\text {thf }}$ é a tensão de limiar do transistor.

Recentemente, indústrias de semicondutores têm optado pela utilização de transistores SOI totalmente depletados ${ }^{37,38,39}$, devido a suas vantagens em relação à tecnologia MOS convencional e SOI parcialmente depletado. Foram obtidos resultados promissores, tais como redução de mais de $60 \%$ no consumo de potência $^{37}$ e redução da tensão de alimentação de circuitos analógicos para $0,5 \mathrm{~V}^{39}$.

\subsubsection{Transistores com canal tensionado}

A constante miniaturização dos dispositivos MOS vem causando problemas de degradação da mobilidade, devidos tanto ao elevado campo elétrico, quanto ao aumento na concentração de dopantes na região de canal do transistor ${ }^{1}$. Uma alternativa tecnológica que tem sido considerada atualmente é o uso de tensão mecânica (strain) sobre o canal do transistor MOS, como meio de aumentar a mobilidade dos transistores e, conseqüentemente, sua corrente de dreno ${ }^{19,20}$. O ganho de mobilidade proporcionado pelo uso de tensão mecânica tem origem na redução da massa efetiva de condução ${ }^{40}$. 
Estudos recentes reportam aumento de até $60 \%$ na mobilidade com a utilização de stress tensor nos transistores SOI nMOS, elevando sua transcondutância e corrente de dreno, sem aumento da corrente de fuga ${ }^{41,42}$.

A tensão mecânica sobre o canal dos transistores pode ser induzida de dois modos diferentes:

a) Através da deposição de uma camada de $\mathrm{Si}_{3} \mathrm{~N}_{4}$ (Contact Etch Stop Layer sCESL) sobre a região de porta do transistor, após sua formação e definição. Essa camada de nitreto induz tensão apenas na direção do comprimento de canal, e é chamada de uniaxial strain ${ }^{43}$. Através desta técnica, obtém-se uma força tensora não uniforme ao longo do canal, agindo principalmente sobre as regiões de fonte e dreno. Desta forma, este tipo de tensão mecânica torna-se mais efetiva à medida que o comprimento de canal é reduzido ${ }^{44}$.

b) Através da utilização de ligas de $\mathrm{Si}_{x} \mathrm{Ge}_{1-\mathrm{x}}$, diminuindo-se gradualmente a concentração de Ge na liga durante o processo de deposição dos materiais (Stress Relaxed Buffer - SRB), até que o material se torne silício puro na parte superior. Posteriormente, esta camada de Si pura sofre um processo de oxidação térmica e é unida a outra lâmina, que já possui uma camada espessa de óxido, por meio de técnicas de união de lâminas (bonded wafers) ${ }^{45}$, originando a lâmina SOI (sSOI). A liga de SiGe é, então, totalmente removida, e o silício resultante preserva a tensão mecânica exercida pela camada de SiGe anteriormente existente. Esta técnica induz tensão mecânica em ambas as direções do canal do transistor e é conhecida como biaxial strain ${ }^{46}$. À medida que o comprimento de canal diminui, a componente de tensão mecânica na direção da corrente sofre uma redução, diferentemente do caso uniaxial $^{44}$. 
2.2 INFLUÊNCIA DA REDUÇÃO DA TEMPERATURA NAS PROPRIEDADES ELÉTRICAS DOS SEMICONDUTORES

Nesta seção será apresentada uma revisão bibliográfica sobre os efeitos causados pela redução da temperatura nas propriedades elétricas dos semicondutores. Modelos analíticos que descrevem estes parâmetros também serão apresentados.

\subsubsection{Concentração intrínseca de portadores e largura da faixa proibida}

A redução da temperatura tem influência direta sobre a concentração intrínseca de portadores, como descrito na eq. $(5)^{47}$ :

$$
n_{i}=2 \cdot\left[\frac{2 \pi m_{0} k}{h^{2}}\right]^{3 / 2} \cdot\left(m_{d e}{ }^{*} m_{d h}^{*}\right)^{3 / 4} \cdot T^{3 / 2} \cdot \exp \left(\frac{-E g}{2 k T}\right)
$$

onde $\mathrm{m}_{0}$ é a massa do elétron, $\mathrm{h}$ é a constante de Planck, $\mathrm{k}$ é a constante de Boltzmann, $m_{d e}{ }^{*} e m_{d h}{ }^{*}$ são as massas efetivas de densidades de estados para elétrons e lacunas, respectivamente ${ }^{47}$, e Eg, a largura da faixa proibida do silício, que também é dependente da temperatura, e pode ser expressa por ${ }^{48}$ :

$$
\begin{aligned}
& \mathrm{Eg}=1,17+1,059 \cdot 10^{-5} \cdot \mathrm{T}-6,05 \cdot 10^{-7} \cdot \mathrm{T}^{2}, \mathrm{~T} \leq 170 \mathrm{~K} \\
& \mathrm{Eg}=1,1785-9,025 \cdot 10^{-5} \cdot \mathrm{T}-3,05 \cdot 10^{-7} \cdot \mathrm{T}^{2}, \mathrm{~T}>170 \mathrm{~K}
\end{aligned}
$$

A eq. (5) também pode ser escrita como

$$
\mathrm{n}_{\mathrm{i}}=\sqrt{\mathrm{N}_{\mathrm{C}} \cdot \mathrm{N}_{\mathrm{V}}} \cdot \exp \left(\frac{-\mathrm{Eg}}{2 \mathrm{kT}}\right)
$$

onde $N_{C}$ e $N_{V}$ são as densidades de estados nas faixas de condução e valência, respectivamente, sendo descritas por:

$$
\mathrm{N}_{\mathrm{C}}=2 \cdot\left(\frac{2 \pi \mathrm{m}_{\mathrm{de}}{ }^{*} \mathrm{~m}_{0} \mathrm{kT} T}{\mathrm{~h}^{2}}\right)^{3 / 2}
$$




$$
N_{v}=2 \cdot\left(\frac{2 \pi m_{d h}{ }^{*} m_{0} k T}{h^{2}}\right)^{3 / 2}
$$

Como pode ser notado, através da eq. (7), a concentração intrínseca de portadores depende apenas do material (através do termo Eg) e da temperatura. Em um semicondutor intrínseco sem a presença de luz, elétrons e lacunas livres podem ser gerados apenas por excitação térmica. Assim, a concentração de portadores em um semicondutor intrínseco é igual a zero para $\mathrm{T}=0 \mathrm{~K}$. Quando a temperatura é elevada, um número cada vez maior de elétrons ganha energia térmica suficiente para se tornar um elétron livre, como pode ser visto na Figura 3, onde é apresentada a curva $n_{i}$ em função da temperatura para o silício. Na mesma figura é também apresentada a largura da faixa proibida em função da temperatura.

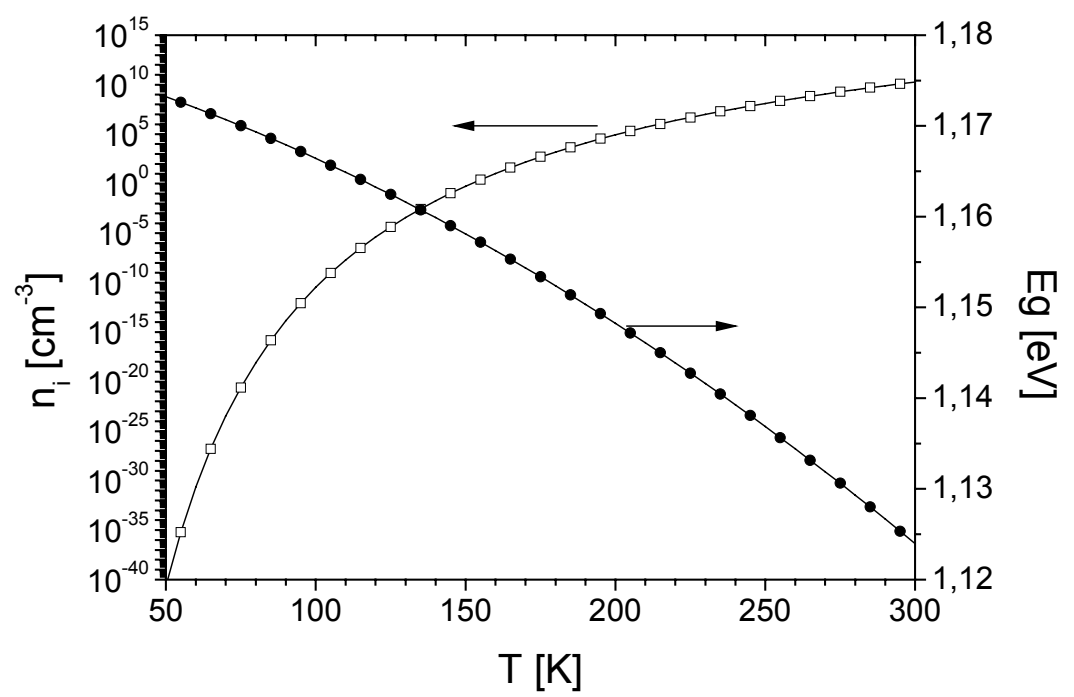

Figura 3 - Concentração intrínseca de portadores e largura da faixa proibida do silício em função da temperatura, calculadas através das eqs. (5) e (6).

Como se pode observar, $\mathrm{n}_{\mathrm{i}}$ apresenta uma enorme redução para menores temperaturas enquanto $\mathrm{Eg}$ sofre um aumento moderado. Os valores de $\mathrm{n}_{\mathrm{i}}$ em temperaturas criogênicas são um grande complicador para a realização de simulações numéricas nestas temperaturas. 


\subsubsection{Ionização incompleta de portadores e Potencial de Fermi}

Quando um semicondutor extrínseco (dopado) é submetido a baixas temperaturas, a energia térmica disponível não é suficiente para ionizar todos os dopantes ${ }^{48}$. Neste caso, a quantidade de impurezas aceitadoras ionizadas, $\mathrm{N}_{\mathrm{A}}^{-}$, pode ser calculada por

$$
\mathrm{N}_{\mathrm{A}}^{-}=\frac{\mathrm{N}_{\mathrm{A}}}{1+4 \cdot \exp \left(\frac{\mathrm{E}_{\mathrm{A}}-\mathrm{E}_{\mathrm{Fp}}}{\mathrm{k} \cdot \mathrm{T}}\right)}
$$

e as impurezas doadoras ionizadas, $\mathrm{N}_{\mathrm{D}}{ }^{+}$, por

$$
\mathrm{N}_{\mathrm{D}}^{+}=\frac{\mathrm{N}_{\mathrm{D}}}{1+2 \cdot \exp \left(\frac{\mathrm{E}_{\mathrm{Fn}}-\mathrm{E}_{\mathrm{D}}}{\mathrm{k} \cdot \mathrm{T}}\right)}
$$

onde $N_{A}$ é a concentração de impurezas aceitadoras, $N_{D}$ é a concentração de impurezas doadoras, $E_{A}$ e $E_{D}$ são os níveis de energia das impurezas e $E_{F n}$ e $E_{F p}$ são os níveis de Fermi para elétrons e lacunas, expressos pelas eqs. (12) e (13), respectivamente ${ }^{49}$.

$$
\begin{aligned}
& E_{F n}=\left(\frac{E_{C}+E_{D}}{2}\right)+\frac{k T}{2} \ln \left(\frac{N_{D}}{2 N_{C}}\right)-k T \sinh ^{-1}\left(\sqrt{\frac{N_{C}}{8 N_{D}}} \exp \left(-\frac{E_{C}-E_{D}}{2 k T}\right)\right) \\
& E_{F p}=\left(\frac{E_{A}+E_{V}}{2}\right)+\frac{k T}{2} \ln \left(\frac{N_{A}}{4 N_{V}}\right)-k T \sinh ^{-1}\left(\sqrt{\frac{N_{V}}{16 N_{A}}} \exp \left(\frac{E_{V}-E_{A}}{2 k T}\right)\right)
\end{aligned}
$$

onde $E_{C}$ e $E_{V}$ são os níveis de energia de condução e valência.

O potencial de Fermi, $\phi_{F}$, é função da temperatura e da quantidade de impurezas ionizadas e, para substrato tipo $p$, pode ser descrito por:

$$
\phi_{\mathrm{F}}=\frac{\mathrm{k} \cdot \mathrm{T}}{\mathrm{q}} \ln \left(\frac{\mathrm{N}_{\mathrm{A}}^{-}}{\mathrm{n}_{\mathrm{i}}}\right)
$$

$\mathrm{Na}$ Figura 4 são apresentadas as curvas da porcentagem de impurezas aceitadoras ionizadas e do potencial de Fermi, considerando silício dopado $\operatorname{com} \mathrm{N}_{\mathrm{A}}=$ $10^{17} \mathrm{~cm}^{-3}$. 


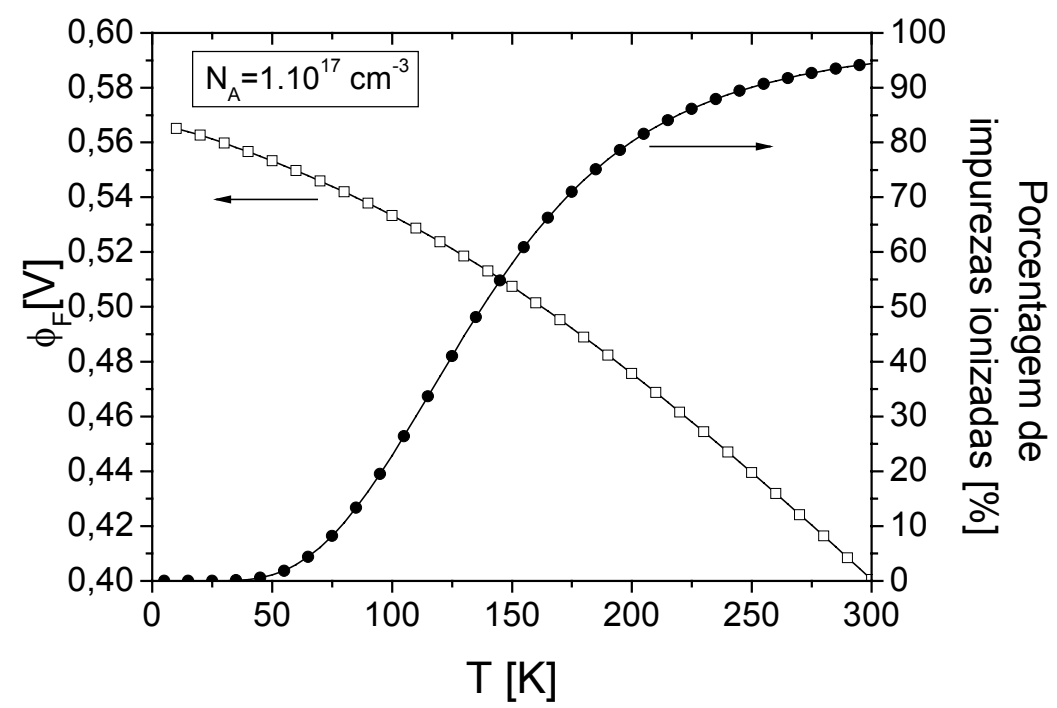

Figura 4 - Porcentagem de impurezas ionizadas e potencial de Fermi em função da temperatura, para um substrato de silício tipo $p$, com $N_{A}=1.10^{17} \mathrm{~cm}^{-3}$.

A partir das curvas apresentadas, pode-se notar que o potencial de Fermi aumenta com a redução da temperatura, apesar do fator proporcional a esta e da redução da porcentagem de impurezas ionizadas. Este aumento deve-se ao fato de que a concentração intrínseca diminui muitas ordens de grandeza (Figura 3) compensando a redução da temperatura. Observa-se também que, em temperaturas muito baixas, uma porcentagem ínfima de impurezas é ionizada.

\subsection{PARÂMETROS ELÉTRICOS DO TRANSISTOR SOI CONVENCIONAL CONSIDERANDO A INFLUÊNCIA DA REDUÇÃO DA TEMPERATURA}

Esta seção apresenta os principais parâmetros elétricos dos transistores SOI, os quais serão utilizados nos capítulos seguintes, enfatizando o comportamento de dispositivos totalmente depletados. Serão apresentadas também alterações decorrentes da redução da temperatura sobre estes parâmetros. 


\subsubsection{Tensão de limiar}

A tensão de limiar $\left(\mathrm{V}_{\mathrm{thf}}\right)$ de um transistor MOSFET pode ser definida como aquela que, aplicada à sua porta, eleva o potencial na superfície da camada de silício para $2 \phi_{\mathrm{F}}$. Para transistores nMOSFETs convencionais, a tensão de limiar pode ser expressa por ${ }^{24}$ :

$$
\mathrm{V}_{\mathrm{thf}}=\mathrm{V}_{\mathrm{FB}}+2 \phi_{\mathrm{F}}+\frac{\mathrm{q} \cdot \mathrm{N}_{\mathrm{A}} \cdot \mathrm{x}_{\mathrm{dmax}}}{\mathrm{C}_{\mathrm{ox}}}
$$

onde a tensão de faixa plana, $\mathrm{V}_{\mathrm{FB}}$ é dada por $\mathrm{V}_{\mathrm{FB}}=\phi_{\mathrm{MS}}-\frac{\mathrm{Q}_{\mathrm{ox}}}{\mathrm{C}_{\mathrm{ox}}} \quad$, sendo $\phi_{\mathrm{MS}} \quad$ a diferença da função trabalho entre o eletrodo de porta e silício e $Q_{\mathrm{ox}}$ a densidade de carga fixa no óxido de porta por unidade de área.

Em transistores SOI parcialmente depletados não há interação entre as regiões de depleção da primeira e segunda interfaces, e a tensão de limiar na primeira interface $\left(\mathrm{V}_{\text {thf }}\right)$ é a mesma de um transistor MOS convencional, calculada pela eq. (15). No caso de transistores SOI totalmente depletados, onde há interação entre as regiões de depleção, as relações entre as tensões aplicadas à porta e ao substrato podem ser expressas pelas equações de Lim \& Fossum ${ }^{34}$, desprezandose as armadilhas de interface:

$$
\begin{aligned}
& V_{G F}=\phi_{M S F}-\frac{Q_{o x f}}{C_{o x f}}+\left(1+\frac{C_{S i}}{C_{\text {oxf }}}\right) \phi_{S F}-\frac{C_{S i}}{C_{o x f}} \phi_{S B}-\frac{\frac{1}{2} Q_{\text {depl }}+Q_{n f}}{C_{o x f}} \\
& V_{G B}=\phi_{M S B}-\frac{Q_{\text {oxb }}}{C_{\text {oxb }}}-\frac{C_{S i}}{C_{\text {oxb }}} \phi_{S F}+\left(1+\frac{C_{S i}}{C_{\text {oxb }}}\right) \phi_{S B}-\frac{\frac{1}{2} Q_{\text {depl }}+Q_{S B}}{C_{o x b}}
\end{aligned}
$$

onde $Q_{o x f}$ e $Q_{n f}$ são, respectivamente, as densidades de carga fixa e de carga de inversão na primeira interface $\left(Q_{n f}<0\right)$ do transistor SOI, $Q_{\text {depl }}$ é a densidade de carga de depleção total na camada de silício, dada por $Q_{\text {depl }}=-\mathrm{qN}_{\mathrm{A}} \mathrm{t}_{\mathrm{Si}}, \mathrm{Q}_{\mathrm{oxb}}$ é $a$ densidade de carga fixa no óxido enterrado e $Q_{S B}$ é a densidade de carga de inversão $\left(Q_{S B}<0\right)$ ou de acumulação $\left(Q_{S B}>0\right)$ na segunda interface, ambos por unidade de área, $\phi_{M S F}$ e $\phi_{M S B}$ são as diferenças de função trabalho entre o eletrodo 
de porta e a camada de silício e entre o substrato e a camada de silício no transistor, respectivamente.

As equações (16) e (17) descrevem o acoplamento entre os potenciais aplicados à porta e ao substrato, que resultam em uma dependência da tensão de limiar do SOI nMOSFET de camada fina com a polarização do substrato, conforme os seguintes casos:

- Segunda interface acumulada $\left(\mathrm{V}_{\mathrm{thf}, \mathrm{accB}}\right)$, onde $\phi_{\mathrm{SB}}=0, \mathrm{Q}_{\mathrm{nf}}=0$ e $\phi_{\mathrm{SF}}=2 \phi_{\mathrm{F}}$ :

$$
\mathrm{V}_{\mathrm{thf}, \mathrm{accB}}=\phi_{\mathrm{MSF}}-\frac{\mathrm{Q}_{\mathrm{oxf}}}{\mathrm{C}_{\mathrm{oxf}}}+\left(1+\frac{\mathrm{C}_{\mathrm{Si}}}{\mathrm{C}_{\mathrm{oxf}}}\right) 2 \phi_{\mathrm{F}}-\frac{\mathrm{Q}_{\mathrm{depl}}}{2 \mathrm{C}_{\mathrm{oxf}}}
$$

- Segunda interface invertida $\left(V_{\mathrm{thf}}\right.$,invB $)$, onde $\phi_{\mathrm{SB}}=2 \phi_{\mathrm{F}}, \mathrm{Q}_{\mathrm{nf}}=0$ e $\phi_{\mathrm{SF}}=2 \phi_{\mathrm{F}}$ :

$$
\mathrm{V}_{\mathrm{thf}, \text { invB }}=\phi_{\mathrm{MSF}}-\frac{\mathrm{Q}_{\mathrm{oxf}}}{\mathrm{C}_{\mathrm{oxf}}}+2 \phi_{\mathrm{F}}-\frac{\mathrm{Q}_{\mathrm{depl}}}{2 \mathrm{C}_{\mathrm{oxf}}}
$$

- Segunda interface depletada $\left(V_{\text {thf, deplB }}\right)$, onde $0<\phi_{\mathrm{SB}}<2 \phi_{\mathrm{F}}, \mathrm{Q}_{\mathrm{nf}}=0$ e $\phi_{\mathrm{SF}}=2 \phi_{\mathrm{F}}$ :

$$
\mathrm{V}_{\text {thf }, \mathrm{deplB}}=\mathrm{V}_{\mathrm{thf}, \mathrm{accB}}-\frac{\mathrm{C}_{\mathrm{Si}} \cdot \mathrm{C}_{\mathrm{oxb}}}{\mathrm{C}_{\mathrm{oxf}}\left(\mathrm{C}_{\mathrm{Si}}+\mathrm{C}_{\mathrm{oxb}}\right)} \cdot\left(\mathrm{V}_{\mathrm{GB}}-\mathrm{V}_{\mathrm{GB}, \mathrm{accB}}\right)
$$

onde, $V_{G B, a c c B}$ é a tensão aplicada ao substrato para que a segunda interface acumule, calculada pela eq. (17) com $\phi_{\mathrm{SB}}=0, \phi_{\mathrm{SF}}=2 \phi_{\mathrm{F}}$ e $\mathrm{Q}_{\mathrm{SB}}=0$.

Vale ressaltar que as eqs. (18), (19) e (20) são válidas somente se a espessura das camadas de inversão e acumulação forem desprezíveis em relação à espessura da camada de silício $^{34}$.

\subsubsection{Variação da tensão de limiar com a temperatura}

Incluindo-se o efeito das armadilhas de interface, a tensão de limiar de um transistor MOS, convencional ou SOI parcialmente depletado, é dada por ${ }^{50}$

$$
\mathrm{V}_{\text {thf }}=\phi_{\mathrm{MS}}-\frac{\mathrm{Q}_{\mathrm{ox}}}{\mathrm{C}_{\mathrm{ox}}}+\frac{\mathrm{q} \cdot \mathrm{N}_{\mathrm{itf}} \cdot \phi_{\mathrm{F}}}{\mathrm{C}_{\mathrm{ox}}}-\frac{\mathrm{Q}_{\text {depl }}}{\mathrm{C}_{\mathrm{ox}}}+2 \phi_{\mathrm{F}}
$$

onde $\mathrm{N}_{\text {iff }}$ é a densidade de armadilhas de interface na primeira interface por unidade 
de área.

Devido à dependência da tensão de limiar com $\phi_{F}$, pode-se notar que, com a redução da temperatura, e conseqüente aumento do potencial de Fermi, a tensão de limiar de dispositivos MOS aumenta. Além disso, com a redução da temperatura, a influência das armadilhas de interface torna-se significativa, contribuindo para o aumento de $\mathrm{V}_{\text {thf. }}$.

Em transistores MOS convencionais e SOI parcialmente depletados, a densidade de carga de depleção, $Q_{d e p l}$, é dada por $Q_{d e p l}=-q N_{A} x_{d \max }$. Desta forma, diferenciando a eq. (21) com relação à $\mathrm{T}$, temos que, para estes transistores, a variação da tensão de limiar com a temperatura pode ser expressa por ${ }^{50}$ :

$$
\frac{\mathrm{dV}_{\text {thf }}}{\mathrm{dT}}=\frac{\mathrm{d} \phi_{\mathrm{F}}}{\mathrm{dT}}\left[1+\alpha_{\mathrm{V}_{\mathrm{T}}} \frac{\mathrm{q}}{\mathrm{C}_{\mathrm{oxf}}}\left(\frac{\varepsilon_{\mathrm{Si}} \mathrm{N}_{\mathrm{A}}}{\mathrm{kT} \ln \left(\mathrm{N}_{\mathrm{A}} / \mathrm{n}_{\mathrm{i}}\right)}\right)^{\frac{1}{2}}+\frac{\mathrm{qN}_{\text {iff }}}{\mathrm{C}_{\text {oxf }}}\right]
$$

onde $\alpha_{V_{T}}=1$.

Como apresentado anteriormente, em transistores SOI totalmente depletados, a espessura da camada de silício é menor que $\mathrm{x}_{\mathrm{dmax}}$, e sua tensão de limiar independe da profundidade da camada de depleção. Desta forma, a carga de depleção torna-se função da espessura da camada de silício, e sua variação com a temperatura pode ser desprezada. Neste caso, a variação da tensão de limiar com a temperatura é dada pela eq. (22) com $\alpha_{V_{T}}=0^{1}$.

Se considerarmos que a influência das armadilhas de interface seja a mesma nos transistores MOS convencionais e SOI, tem-se que a variação de $V_{\text {thf }}$ com a temperatura é menor nos dispositivos SOI totalmente depletados do que nos dispositivos MOS convencionais e SOI de camada espessa. A ref. [32] reporta variações, em valor absoluto, da ordem de $0,75 \mathrm{mV} / \mathrm{K}$ para transistores totalmente depletados e maior que $2 \mathrm{mV} / \mathrm{K}$ para transistores MOS convencionais.

Quando a temperatura é reduzida, dispositivos parcialmente depletados podem se tornar totalmente depletados devido ao aumento do potencial de Fermi e conseqüente aumento da região de depleção. Neste caso, uma significativa redução em $\mathrm{dV}_{\text {thf }} / \mathrm{dT}$ pode ser obtida em dispositivos SOI totalmente depletados, uma vez que a variação da região de depleção com a temperatura é suprimida. 


\subsubsection{Inclinação de sublimiar}

Define-se a inclinação de sublimiar (S) de transistores MOS como a variação de tensão de porta necessária para aumentar em uma década a corrente de dreno na região de sublimiar ${ }^{50}$.

Desta maneira, a inclinação de sublimiar é dada por:

$$
S=\frac{d V_{G F}}{d\left(\log I_{D S}\right)}
$$

Utilizando a equação da corrente de dreno em sublimiar, que é composta predominantemente pela componente de difusão ${ }^{24}$, e após algumas simplificações, obtém-se $^{51}$ :

$$
S=\frac{k T}{q} \ln (10)(1+\alpha)=\mathrm{n} \frac{k T}{q} \ln (10)
$$

A partir desta equação, pode-se notar que a inclinação de sublimiar depende diretamente do fator de corpo do transistor. Como apresentado na seção 2.1.2, transistores SOI totalmente depletados apresentam menor fator de corpo em comparação com dispositivos convencionais ${ }^{1}$. Como resultado, temos que:

$$
\mathrm{S}_{\text {sOl totalmente depletado }}<\mathrm{S}_{\mathrm{MOS}} \text { convencional }<\mathrm{S}_{\text {SOI com a segunda interface acumulada }}
$$

Estes menores valores de inclinação de sublimiar dos transistores SOI totalmente depletados permitem a utilização de transistores com tensões de limiar menores que do transistor MOS convencional, sem aumento da corrente de fuga, possibilitando melhor desempenho em alta velocidade, principalmente com baixa tensão de alimentação ${ }^{52}$.

Uma das principais vantagens da operação de transistores MOS em baixa temperatura está relacionada à redução da inclinação de sublimiar. Considerando-se as armadilhas de interface, o fator de acoplamento capacitivo do transistor MOS, $\alpha$, na eq. (24), é dado por:

$$
\alpha=\frac{C_{D}+C_{\text {iff }}}{C_{\text {ox }}}
$$

para transistores MOS convencionais e 


$$
\alpha=\frac{C_{\text {itf }}}{C_{\text {oxf }}}+\frac{C_{S i}}{C_{\text {oxf }}}-\frac{\frac{C_{S i}^{2}}{C_{o x f} \cdot C_{o x b}}}{1+\frac{C_{i t b}}{C_{\text {oxb }}}+\frac{C_{S i}}{C_{o x b}}}
$$

para transistores SOI totalmente depletados.

Nas expressões acima, $\mathrm{C}_{\text {itf }}$ e $\mathrm{C}_{\text {itb }}$ são, respectivamente, as capacitâncias das armadilhas de interface da primeira e segunda interfaces, $C_{i f f}=q \cdot N_{i f f}$ e $C_{i t b}=q \cdot N_{i t b}$, onde $\mathrm{N}_{\text {itf }}$ e $\mathrm{N}_{\text {itb }}$ são as densidades de armadilhas de interface na primeira e segunda interfaces.

Como pode ser observado através da eq. (24), a inclinação de sublimiar apresenta um fator proporcional à temperatura. Assim, dever-se-ia esperar que qualquer diminuição da temperatura provocasse a redução de S. Reduzindo-se a temperatura, entretanto, a influência da densidade de armadilhas de interface tornase apreciável, fazendo com que os valores de $\mathrm{C}_{\text {itf }}$ e $\mathrm{C}_{\text {itb }}$ se elevem rapidamente, provocando o aumento de $\mathrm{S}$, ao contrário da esperada redução. Em dispositivos totalmente depletados, são observados valores de inclinação de sublimiar de 23 $\mathrm{mV} /$ década para temperatura de $9 \mathrm{~K}^{1}$.

\subsubsection{Efeitos de canal curto}

Diversos efeitos são causados pela redução do comprimento de canal em transistores $\mathrm{MOS}^{53}$. Com a redução do comprimento de canal, as regiões de depleção de fonte e dreno tornam-se significativas em relação à região de depleção induzida pela tensão aplicada à porta. Desta forma, a carga de depleção controlada pela porta não pode mais ser expressa por $Q_{\text {depl }}=-q N_{A} x_{d \max }$, como no caso do transistor MOS convencional, mas sim como uma fração dela ( $\left.Q_{\text {depl,ef }}\right)$. Uma vez que $Q_{\text {depl,ef }}<Q_{\text {depl, }}$ haverá uma redução na tensão de limiar e um aumento da inclinação de sublimiar $24,54,55$.

Os dispositivos SOI de camada fina sofrem menor influência da redução do comprimento de canal do que os dispositivos MOS convencionais, uma vez que a 
porcentagem da carga total que é efetivamente controlada pela porta em dispositivos SOI de canal curto é maior quando comparada aos dispositivos convencionais.

O efeito de redução de barreira induzida pelo dreno (Drain-Induced Barrier Lowering - DIBL), ocorre também devido ao compartilhamento de cargas entre a porta e as junções ${ }^{53}$. As regiões de fonte e dreno de um transistor MOS formam junções PN com o corpo do dispositivo. A largura das regiões de depleção associadas a essas junções aumenta com a polarização reversa promovida pela tensão aplicada ao dreno. Em um dispositivo MOSFET, se o canal é suficientemente longo, ao aplicar-se uma tensão ao dreno, não há alteração na barreira de potencial da junção de fonte. Por outro lado, em um dispositivo de canal curto, a barreira de potencial na fonte pode ser reduzida, dependendo da polarização de dreno. Assim, o aumento da região de depleção de dreno, causado pelo aumento da tensão entre fonte e dreno, $V_{D S}$, faz com que a carga de depleção controlada pela porta seja reduzida, provocando, assim, a redução da tensão de limiar para altos valores de VDS.

Estudos apontam que a tensão de limiar de dispositivos SOI sofre variação significativa quando a tensão de dreno aumenta, devido à ativação da estrutura bipolar parasitária, resultando em maior DIBL nestes dispositivos em comparação com dispositivos convencionais ${ }^{56}$.

O efeito de DIBL pode ser incluído no cálculo da tensão de limiar através da expressão abaixo, onde $\sigma$ é o parâmetro que considera este efeito e $V_{\text {thfo }}$ é a tensão de limiar de um dispositivo de canal longo ${ }^{55}$.

$$
\mathrm{V}_{\mathrm{thf}}=\mathrm{V}_{\mathrm{thf} 0}-\sigma \mathrm{V}_{\mathrm{DS}}
$$

A redução da temperatura tende a reduzir a ocorrência de efeitos de canal curto, devido à redução da porcentagem da carga total que sofre influência das regiões de depleção de fonte e dreno ${ }^{57}$.

Em baixas temperaturas, o efeito de DIBL tende a ser menos acentuado, ou no mínimo igual, àquele observado em temperatura ambiente ${ }^{57,58}$, exceto com altas concentrações de dopantes na região do canal, como pode ocorrer em dispositivos de canal curto com implantação de halo, nos quais o DIBL aumenta em relação a temperatura ambiente devida ao efeito de ionização por impacto com altas polarizações de dreno em baixas temperaturas ${ }^{58}$. 


\subsubsection{Mobilidade}

A mobilidade dos portadores é um fator bastante importante para a determinação da capacidade de fornecimento de corrente de um SOI MOSFET. O movimento dos portadores em um cristal semicondutor não é livre, mas limitado por mecanismos de espalhamento que reduzem sua mobilidade. Alguns destes mecanismos de degradação são fortemente dependentes da temperatura, e podem ser classificados como: espalhamento de rede, espalhamento por impurezas ionizadas, espalhamento portador-portador e espalhamento por impurezas neutras. Nesta seção, serão apresentados os modelos analíticos que descrevem cada um destes mecanismos de espalhamento de portadores mencionados e sua dependência com a temperatura.

\section{a) Espalhamento de rede ou espalhamento por fônons (lattice scattering)}

Este mecanismo de espalhamento está relacionado às interações entre os portadores e as vibrações da rede cristalina (fônons). A redução da temperatura provoca a diminuição destas vibrações, causando aumento da mobilidade.

O cálculo da mobilidade dos portadores sofrendo deste mecanismo de espalhamento, $\mu_{\mathrm{ps}}$, não é simples, uma vez que envolve o cálculo da energia dos fônons e a energia interna do cristal deve ser conhecida. Dentre uma série de modelos matemáticos propostos para o cálculo de $\mu_{\mathrm{ps}}$, o modelo mais utilizado foi proposto por Sah et al ${ }^{59}$ :

$$
\mu_{\mathrm{pse}, \mathrm{psh}}=\frac{1}{\left(\frac{1}{\mu_{0 \mathrm{ea}, 0 \mathrm{ha}}\left(\frac{\mathrm{T}}{300}\right)^{-\alpha \mathrm{e},-\alpha \mathrm{h}}}+\frac{1}{\mu_{0 \mathrm{eb}, 0 \mathrm{hb}}\left(\frac{\mathrm{T}}{300}\right)^{-\beta \mathrm{e},-\beta \mathrm{h}}}\right)}
$$

onde o índice e refere-se a elétrons e $h$, às lacunas. 
Na equação acima, $\mu_{0 e a}=4195 \mathrm{~cm}^{2} / \mathrm{Vs} ; \mu_{0 \mathrm{ha}}=2502 \mathrm{~cm}^{2} / \mathrm{Vs} ; \mu_{0 \mathrm{eb}}=2153 \mathrm{~cm}^{2} / \mathrm{Vs}$; $\mu_{0 h b}=591 \mathrm{~cm}^{2} / \mathrm{ss} ; \alpha_{e}=\alpha_{h}=1,5 ; \beta_{e}=3,13$ e $\beta_{h}=3,25$.

Neste modelo, fônons ópticos e acústicos são combinados utilizando a regra de Mathiessen ${ }^{47}$.

\section{b) Espalhamento por impurezas ionizadas (ionized impurity scattering)}

Este mecanismo de espalhamento está relacionado às altas concentrações de dopantes, que causam a redução da mobilidade dos portadores.

Apesar de existir um modelo analítico bastante preciso para o cálculo da mobilidade devido ao espalhamento por impurezas ionizadas, $\mu_{\mathrm{ii}}{ }^{60,61}$, este não pode ser combinado ao espalhamento de rede, utilizando a regra de Mathiessen, pois estes mecanismos não são totalmente independentes. Entretanto, Caughey e Thomas ${ }^{62}$ propuseram um modelo empírico que inclui estes dois mecanismos em uma única equação que, para elétrons, é escrita como:

$$
\mu_{\mathrm{psiie}}=\mu_{\mathrm{min}, \mathrm{e}}+\frac{\mu_{\mathrm{pse}}-\mu_{\mathrm{min}, \mathrm{e}}}{1+\left(\frac{\mathrm{N}_{\mathrm{A}}^{-}}{\mathrm{N}_{\text {ref }, \mathrm{e}}}\right)^{\alpha_{\mathrm{ae}}}}
$$

onde $\mu_{\text {min, },}=197,17-45,505 \cdot \log (T) ; N_{\text {ref,e }}=1,12 \cdot 10^{17} \cdot\left(\frac{T}{300}\right)^{3,2}$ e $\alpha_{a e}=0,72 \cdot\left(\frac{T}{300}\right)^{0,065}$.

Para lacunas, o índice $e$ deve ser substituído pelo índice $h \quad \mathrm{e}$ $\mu_{\text {min, } h}=110,90-25,597 \cdot \log (T) ; \mathrm{N}_{\text {ref }, \mathrm{h}}=2,23 \cdot 10^{17} \cdot\left(\frac{\mathrm{T}}{300}\right)^{3,2}$ e $\alpha_{\mathrm{ah}}=\alpha_{\mathrm{ae}}$

\section{c) Espalhamento portador-portador (carrier-to-carrier scattering)}

O mecanismo de espalhamento portador-portador tem grande influência na mobilidade para altas densidades de portadores, como no caso de dispositivos de potência, quando a quantidade de portadores torna-se muito maior que a densidade 
de dopantes ${ }^{63}$. Este tipo de espalhamento também pode ocorrer em dispositivos submicrométricos, nos quais a densidade de corrente pode ser bastante alta.

A mobilidade levando em consideração o espalhamento portador-portador, pode ser modelada como ${ }^{63}$ :

$$
\mu_{\mathrm{CC}}=\frac{2 \cdot 10^{17}}{\sqrt{\mathrm{N}_{\mathrm{A}}} \cdot \ln \left(1+8,28 \cdot 10^{8} \cdot \mathrm{T}^{2} \cdot \mathrm{N}_{\mathrm{A}}^{-\frac{1}{3}}\right)}
$$

Para semicondutor tipo $n$, deve-se apenas substituir $N_{A}$ por $N_{D}$ na eq. (30).

\section{d) Espalhamento por impurezas neutras (neutral-impurity scattering)}

O mecanismo de espalhamento por impurezas neutras deve-se às impurezas não-ionizadas em baixas temperaturas. Este tipo de espalhamento, que só é importante para concentrações de impurezas neutras acima de $10^{18} \mathrm{~cm}^{-3}$ e em baixas temperaturas, pode ser considerado na modelagem da mobilidade através da seguinte expressão (para elétrons) ${ }^{64}$ :

$$
\mu_{\mathrm{ni}}=\mathrm{C}_{0} \cdot\left[\frac{2}{3} \sqrt{\frac{\mathrm{kT}}{\mathrm{E}_{\mathrm{ni}, \mathrm{e}}}}+\frac{1}{3} \sqrt{\frac{\mathrm{E}_{\mathrm{ni}, \mathrm{e}}}{\mathrm{kT}}}\right]
$$

onde $\mathrm{C}_{0}=\left(\frac{2 \pi^{3} \mathrm{q}^{3} \mathrm{~m}_{\mathrm{ce}}{ }^{*}}{5 \varepsilon_{\mathrm{Si}} \mathrm{h}^{3}\left(\mathrm{~N}_{\mathrm{A}}-\mathrm{N}_{\mathrm{A}}^{-}\right)}\right) ; \mathrm{E}_{\mathrm{ni, \textrm {e }}}=1,136 \cdot 10^{-19} \cdot\left(\frac{\mathrm{m}_{\mathrm{ce}}{ }^{*}}{\mathrm{~m}_{0}}\right) \cdot\left(\frac{\varepsilon_{0}}{\varepsilon_{\mathrm{Si}}}\right)$ e $\varepsilon_{0}$ e $\varepsilon_{\mathrm{Si}}$ são as permissividades elétricas do vácuo e do semicondutor, respectivamente e $\mathrm{m}_{\mathrm{ce}}{ }^{*}$, a massa efetiva de condução para elétrons ${ }^{47}$.

Para o cálculo da mobilidade de lacunas levando em conta o espalhamento por impurezas neutras deve-se apenas substituir a massa efetiva de condução para elétrons pela massa efetiva de condução para lacunas, $m_{c h}{ }^{*}$, no cálculo de $C_{0}$ e $E_{n i}$, na eq. (31). 


\section{e) Mobilidade dos portadores independente de campo elétrico}

Os mecanismos de espalhamento apresentados até o momento são independentes das tensões aplicadas e podem ser combinados através da regra de Mathiessen, fornecendo o valor da mobilidade independente de campo elétrico, $\mu_{0}$ :

$$
\mu_{0}=\left(\frac{1}{\frac{1}{\mu_{\mathrm{psii}}}+\frac{1}{\mu_{\mathrm{cc}}}+\frac{1}{\mu_{\mathrm{ni}}}}\right)
$$

$\mathrm{Na}$ Figura 5 são apresentadas as curvas correspondentes às diversas componentes de mobilidade apresentadas, bem como da mobilidade independente do campo elétrico, em função da temperatura.

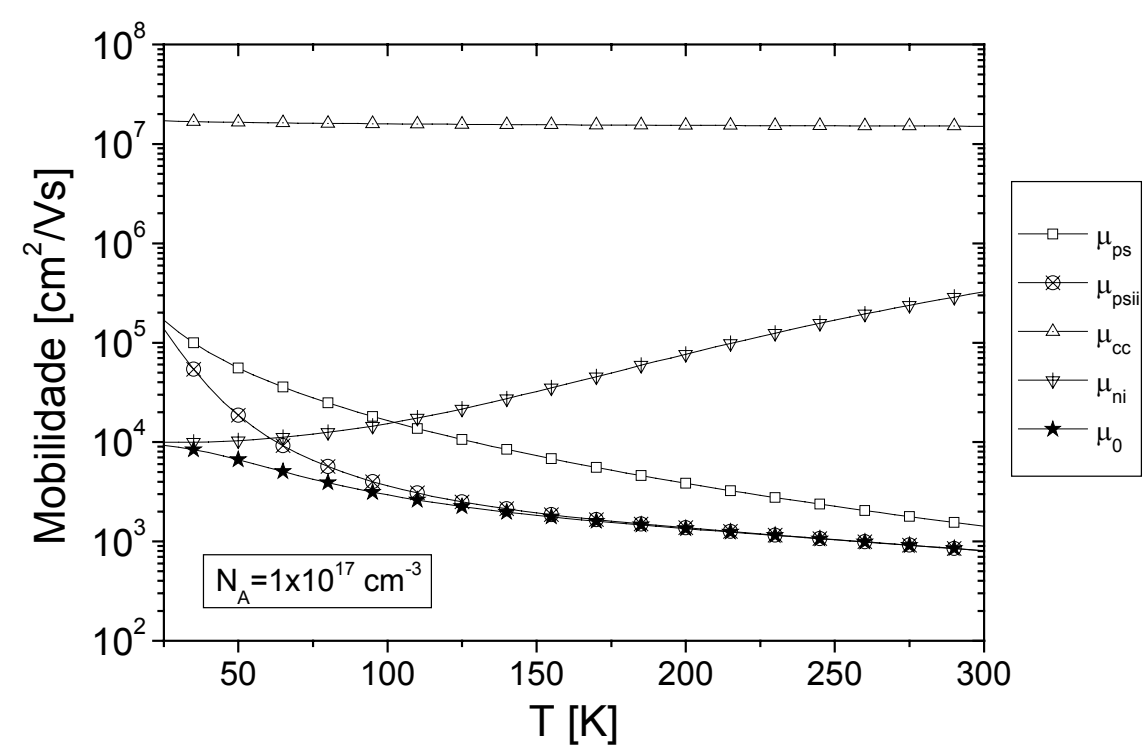

Figura 5 - Curvas das diversas componentes que compõem a mobilidade de elétrons independente de campo elétrico em função da temperatura, para substrato de silício tipo $p$ dopado com $\mathrm{N}_{\mathrm{A}}=1.10^{17} \mathrm{~cm}^{-3}$.

Na Figura 6 são apresentados os resultados para a mobilidade independente de campo elétrico, $\mu_{0}$, em função da temperatura para diferentes concentrações de dopantes. Conforme pode ser visto nesta figura, o aumento da concentração de dopantes provoca a redução do ganho de mobilidade devido à redução da temperatura. Para concentrações de dopantes mais elevadas (maior que $1 \times 10^{18}$ 
$\mathrm{cm}^{-3}$ ), a mobilidade decresce com a redução da temperatura, devido ao espalhamento por impurezas neutras, que se torna mais pronunciado, conforme mencionado anteriormente.

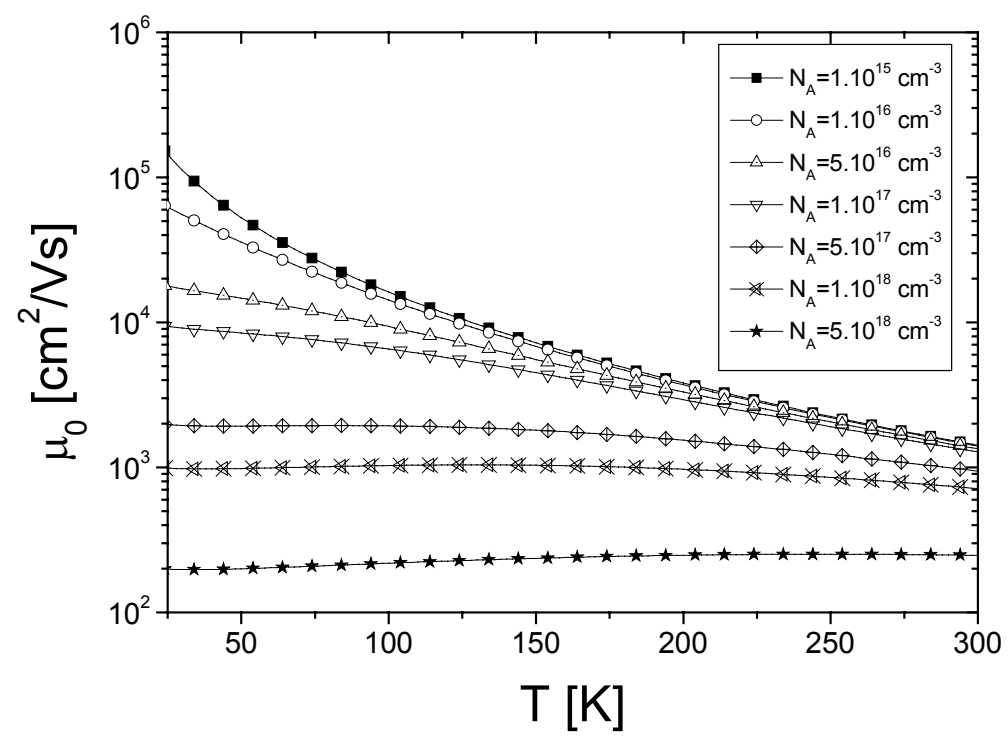

Figura 6 - Mobilidade de elétrons independente de campo elétrico em função da temperatura, para substrato de silício tipo p e diferentes concentrações de dopantes.

\section{f) Mobilidade dos portadores dependente de campo elétrico}

A mobilidade, $\mu_{\mathrm{n}}$, usada nos modelos de transistores MOS não é a mobilidade dos elétrons independente do campo elétrico em um cristal de silício. O campo elétrico vertical (ou transversal), controlado pela tensão aplicada à porta de um transistor MOS, atrai os portadores para a interface entre o semicondutor e o óxido de porta, causando a redução da mobilidade devido à rugosidade superficial. Além disso, quando os portadores são submetidos a um campo elétrico lateral, sua mobilidade também é reduzida, devido à saturação de sua velocidade.

Os transistores SOI totalmente depletados apresentam menor campo elétrico e, conseqüentemente, a mobilidade efetiva destes dispositivos é maior, quando comparado a dispositivos MOS convencionais ${ }^{65}$.

Existem diversos modelos propostos para o cálculo da mobilidade sob a influência de campo elétrico. Neste trabalho, para descrever a mobilidade dos portadores em transistores SOI MOS sob influência do campo elétrico, será utilizado 
o modelo descrito na ref. [55]. A mobilidade degradada devido ao campo elétrico é calculada usando a seguinte equação:

$$
\mu_{\mathrm{n}}=\frac{\mu_{0}}{1+\alpha_{\mathrm{s}}\left|\mathrm{E}_{\text {eff }}\right|}
$$

onde, $\alpha_{S}$ é a constante de espalhamento e $E_{\text {eff }}$ é o campo elétrico médio no canal tanto na direção vertical quanto lateral ${ }^{1}$, descrito por:

$$
\mathrm{E}_{\mathrm{eff}}=\frac{2 \phi_{\mathrm{F}}+\left(\mathrm{V}_{\mathrm{S}}+\mathrm{V}_{\mathrm{DE}}\right) / 2}{\mathrm{t}_{\mathrm{Si}}}+\mathrm{B}-\frac{\left(\mathrm{Q}_{\mathrm{S}}+\mathrm{Q}_{\mathrm{D}}\right) / 2}{2 \varepsilon_{\mathrm{Si}}}
$$

onde $B=-\frac{Q_{\text {depl }}}{2 C_{\text {oxb }} t_{\mathrm{Si}}}\left(\frac{1}{1+C_{S_{i}} C_{\text {oxb }}}+1\right)-\frac{V_{G B}}{\left(1+C_{S i} / C_{o \times b}\right) t_{\text {oxb }}}, V_{D E}$ é o potencial efetivo no canal próximo ao dreno, e $Q_{S}$ e $Q_{D}$ são as densidades de carga de inversão por unidade de área na fronteira do canal com as regiões de fonte e dreno, respectivamente, sendo estes três parâmetros discutidos posteriormente.

De acordo com a eq. (34), pode-se notar que o campo elétrico é dependente da temperatura, através do termo $\phi_{F}$. $O$ aumento de $\phi_{F} e$, conseqüentemente, do campo elétrico é responsável pela maior degradação da mobilidade observada em baixas temperaturas ${ }^{47}$.

\subsubsection{Velocidade de saturação do portador}

Como mencionado anteriormente, os portadores de carga, sob influência de campo elétrico lateral, tem sua mobilidade reduzida e limitada pela velocidade de saturação.

A velocidade de saturação dos portadores é dependente da temperatura, e pode ser expressa por ${ }^{66}$ :

$$
\mathrm{v}_{\text {sat }, \mathrm{e}}=10^{7} \cdot\left(\frac{\mathrm{T}}{300}\right)^{-0,87}[\mathrm{~cm} / \mathrm{s}]
$$

para elétrons e

$$
\mathrm{v}_{\mathrm{sat}, \mathrm{h}}=8,37 \cdot 10^{6} \cdot\left(\frac{\mathrm{T}}{300}\right)^{-0,52}[\mathrm{~cm} / \mathrm{s}]
$$

para lacunas. 


\subsubsection{Transcondutância}

A transcondutância de um transistor MOS, $g_{m}$, mede a eficácia do controle da tensão de porta sobre a corrente de dreno e é dada por:

$$
g_{m}=\frac{d l_{D S}}{d V_{G S}}
$$

As equações para a transcondutância de transistores SOI de camada fina, podem ser obtidas através da diferenciação das equações de corrente descritas pelo modelo de Lim \& Fossum ${ }^{36}$, em triodo e saturação (eqs. (3) e (4)), respectivamente:

$$
\begin{gathered}
g_{m}=\frac{d l_{D S}}{d V_{G F}}=\mu_{n} C_{\text {oxf }} \frac{W}{L} V_{D S} \\
g_{m}=\frac{d l_{D S s a t}}{d V_{G F}}=\frac{\mu_{n} C_{o x f}}{n} \frac{W}{L}\left(V_{G F}-V_{\text {thf }}\right)
\end{gathered}
$$

onde $\mathrm{n}$ é o fator de corpo, descrito na seção 2.1.2. Como resultado do menor fator de corpo, maior transcondutância é observada em transistores SOI totalmente depletados, na região de saturação, do que em dispositivos MOS convencionais e ainda maior do que em um dispositivo SOI de camada fina com a segunda interface acumulada.

Como pode ser visto através das eqs. (38) e (39), a transcondutância está diretamente relacionada à mobilidade dos portadores. A redução da temperatura aumenta a mobilidade e, conseqüentemente $g_{m}$, até $150 \mathrm{~K}$. Em seguida, $g_{m}$ sofre uma redução, causada pelo aumento do campo elétrico, que degrada a mobilidade dos portadores ${ }^{47}$ através do espalhamento de superfície. Estes dois efeitos podem ser vistos através da Figura 7, que apresenta as curvas de transcondutância em função da tensão de porta, resultantes de simulações numéricas de dispositivos SOI totalmente depletados com $\mathrm{L}=0,5 \mu \mathrm{m}$, com $\mathrm{V}_{\mathrm{DS}}=100 \mathrm{mV}$, em diferentes temperaturas. As demais características dos dispositivos simulados são: $t_{\text {oxf }}=15 \mathrm{~nm}$, $t_{\mathrm{Si}}=50 \mathrm{~nm}, \mathrm{t}_{\mathrm{oxb}}=390 \mathrm{~nm}$ e $\mathrm{N}_{\mathrm{AH}}=1,2 \times 10^{17} \mathrm{~cm}^{-3}$. Maiores detalhes a respeito das simulações numéricas realizadas serão descritos ao longo deste trabalho. 


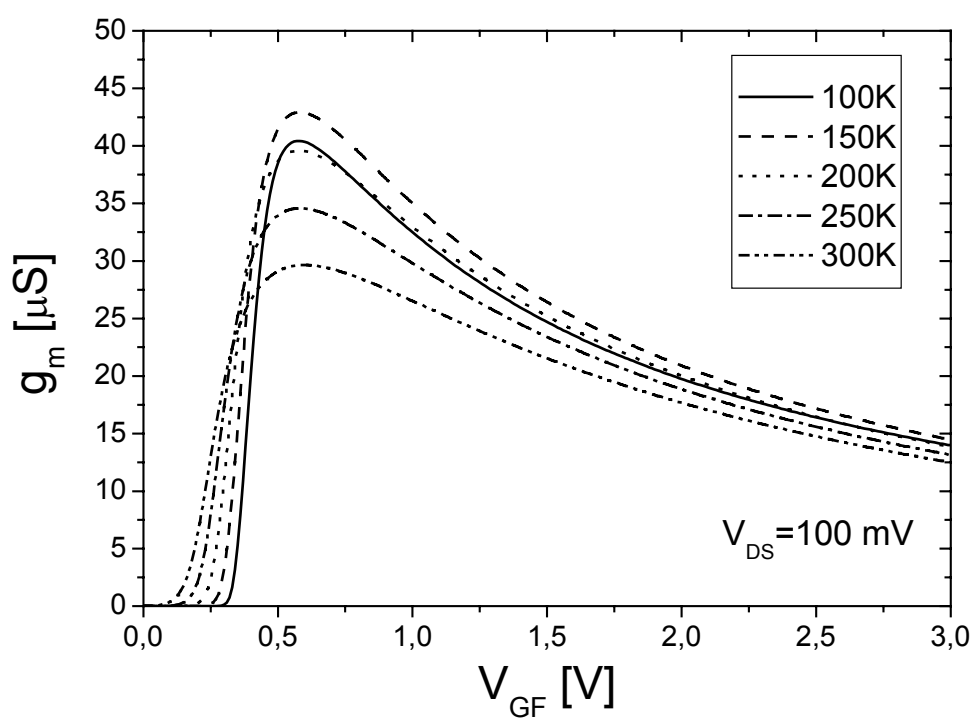

Figura 7 - Transcondutância em função da tensão de porta, simulada para um dispositivo SOI totalmente depletado com $L=0,5 \mu \mathrm{m}$ e $V_{D S}=100 \mathrm{mV}$, em diferentes temperaturas.

\subsubsection{Ionização por impacto}

Em transistores MOS, ao se aplicar uma tensão de dreno alta, os elétrons da região do canal podem adquirir energia suficiente, na região próxima ao dreno, para, através do impacto com a rede cristalina, gerar pares elétron-lacuna. Particularmente em dispositivos SOI totalmente depletados, os elétrons migram rapidamente para a região de dreno (maior potencial), compondo uma parcela adicional da corrente de dreno, enquanto as lacunas migram para a região de menor potencial que, neste caso, é a região de fonte. Uma vez que nestes dispositivos a junção fonte-corpo já está diretamente polarizada devido à depleção da camada de silício, as lacunas podem recombinar-se na fonte, sem aumentar sensivelmente o potencial do corpo, ao contrário do que ocorre em transistores parcialmente depletados ${ }^{1}$.

O aumento do nível de corrente causado pela ionização por impacto pode levar à ruptura prematura da junção ou à perda de controle da porta sobre a corrente. Devido à presença do transistor bipolar parasitário com base flutuante, em transistores $\mathrm{SOI}$, estes apresentam tensão de ruptura menor em relação aos dispositivos MOS convencionais ${ }^{11}$. 
Em temperaturas reduzidas, com uma menor quantidade de portadores na região de canal (devida à ionização incompleta das impurezas) e com menor vibração da rede cristalina, ocorre o aumento da mobilidade. Este aumento de mobilidade, com conseqüente aumento da energia dos portadores, favorece a geração de pares elétron-lacuna. Desta forma, a parcela de corrente proveniente da ionização por impacto aumenta consideravelmente em baixas temperaturas, causando o aumento da condutância de dreno na região de saturação e conseqüente redução da tensão Early, que são parâmetros importantes do ponto de vista analógico e serão tratados na seção 2.4.2.

Para o cálculo da corrente total, incluindo a parcela gerada por ionização por impacto pode-se utilizar as seguintes expressões ${ }^{67}$ :

$$
\begin{gathered}
\mathrm{I}_{\mathrm{DS}, \text { total }}=\mathrm{I}_{\mathrm{i}} \cdot(\mathrm{M}-1)+\mathrm{I}_{\mathrm{DS}} \\
\mathrm{I}_{\mathrm{i}}=\mathrm{I}_{\mathrm{DS}} \cdot(\mathrm{M}-1)
\end{gathered}
$$

sendo

$$
\mathrm{M}-1=\frac{\alpha_{\mathrm{M}}}{\beta_{\mathrm{M}}}\left(\mathrm{V}_{\mathrm{DS}}-\mathrm{V}_{\mathrm{DS}, \mathrm{SAT}}\right) \exp \left(\frac{-\beta_{\mathrm{M}}}{\mathrm{A}\left(\mathrm{V}_{\mathrm{DS}}-\mathrm{V}_{\mathrm{DS}, \mathrm{SAT}}\right)}\right)
$$

onde A é um parâmetro de ajuste ${ }^{67,68}$, dependente do processo de fabricação, e

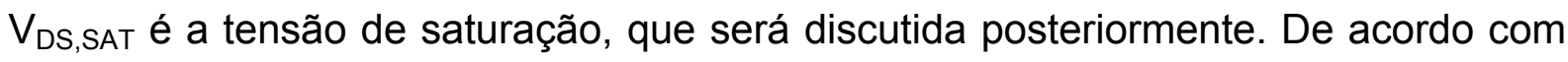
a ref. [69], o parâmetro $\beta_{M}$ é praticamente independente da temperatura, enquanto o parâmetro $\alpha_{M}$ aumenta com a redução da temperatura, como pode ser visto na Figura 8, que apresenta a variação dos parâmetros $\alpha_{M}$ e $\beta_{M}$ com a temperatura, extraídos para transistores MOS convencionais (canal n e p). 

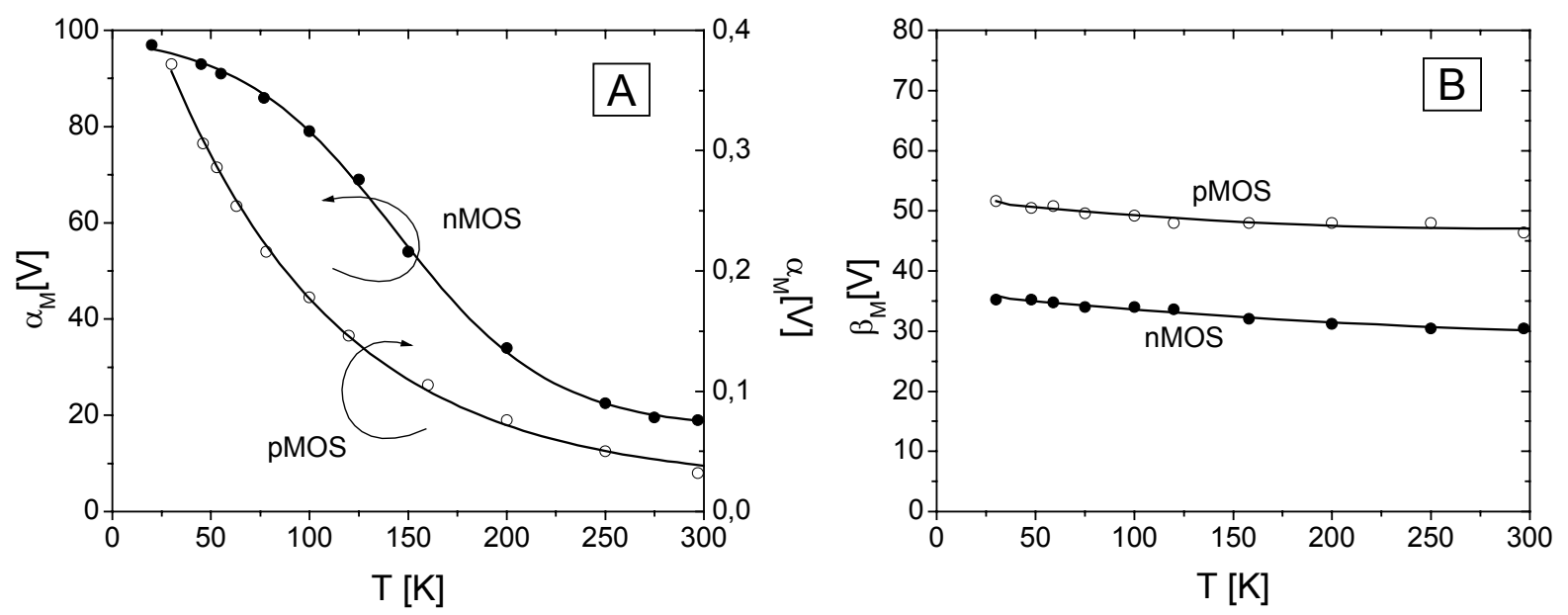

Figura 8 - Variação dos parâmetros $\alpha_{M}$ e $\beta_{M}$ com a temperatura ${ }^{69}$.

\subsubsection{Auto-Aquecimento}

Além das alterações provocadas pela temperatura descritas anteriormente, transistores SOI sofrem de um efeito denominado Auto-Aquecimento.

Transistores SOI são termicamente isolados do substrato pela presença do óxido enterrado, que possui condutividade térmica 100 vezes inferior à do substrato de silício $^{70}$. Como resultado, a remoção do calor gerado no dispositivo pela passagem de corrente é menor do que em transistores MOS convencionais, provocando a elevação da temperatura de funcionamento do transistor à medida que as tensões de polarização são aumentadas ${ }^{71,72}$. Em elevados níveis de potência, este efeito pode causar uma região de condutância negativa na região de saturação, devido à redução da mobilidade causada pelo aquecimento. Este fenômeno torna-se mais crítico quando os dispositivos SOI operam em baixas temperaturas ${ }^{73}$, devido à maior intensidade de corrente decorrente do aumento da mobilidade ${ }^{71}$.

O calor gerado pode se propagar de um dispositivo para outro, e o acoplamento térmico resultante pode afetar o funcionamento de circuitos analógicos sensíveis a variações, tais como espelhos de corrente ${ }^{74}$.

A Figura 9 apresenta curvas da corrente de dreno em função da tensão de dreno obtidas através da simulação numérica de um transistor de canal gradual com 
$\mathrm{L}=0,5 \mu \mathrm{m}$ e $\mathrm{L}_{\mathrm{LD}} / \mathrm{L}=0,2$, em $\mathrm{T}=150 \mathrm{~K}$ com $\mathrm{V}_{\mathrm{GT}}=\mathrm{V}_{\mathrm{GF}}-\mathrm{V}_{\mathrm{thf}}=200 \mathrm{mV}$ e 2,0 V, com e sem o modelo que considera a variação da temperatura da rede cristalina. As demais características dos dispositivos simulados são: $\mathrm{t}_{\mathrm{oxf}}=15 \mathrm{~nm}, \mathrm{t}_{\mathrm{si}}=50 \mathrm{~nm}, \mathrm{t}_{\mathrm{oxb}}=$ $390 \mathrm{~nm}$ e $\mathrm{N}_{\mathrm{AH}}=1,2 \times 10^{17} \mathrm{~cm}^{-3}$. Na mesma figura são apresentadas as curvas da condutância de dreno, g $g_{D}$, obtidas através da diferenciação numérica das curvas de corrente.

A partir das curvas apresentadas, pode-se notar a ocorrência do autoaquecimento para $\mathrm{V}_{\mathrm{GT}}=2,0 \mathrm{~V}$, através da redução da corrente de dreno (Figura 9(A)), provocada pelo aumento da temperatura, que resulta em uma região de condutância de dreno negativa (Figura 9(B)). Além disso, pode-se verificar que o efeito de autoaquecimento é desprezível, mesmo em baixas temperaturas, para transistores com estes parâmetros tecnológicos e baixos valores de sobre-tensão de condução ${ }^{71}\left(\mathrm{~V}_{\mathrm{GT}}\right.$ $=V_{G F}-V_{\text {thf }}$, onde $V_{G F}$ é a tensão aplicada à porta do transistor e $V_{\text {thf }}$ a tensão de limiar). Desta forma, negligenciaremos a ocorrência deste efeito no decorrer deste trabalho.
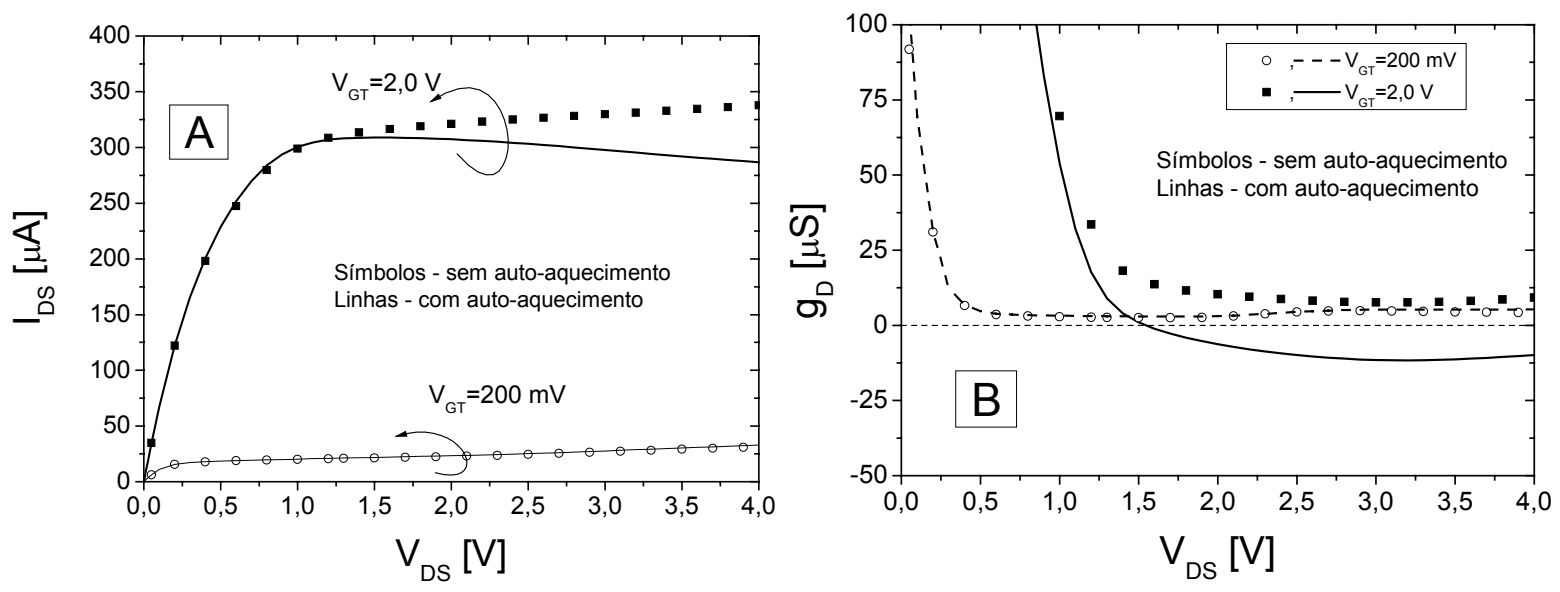

Figura 9 - Corrente de dreno $(A)$ e condutância de dreno $(B)$ em função da tensão de dreno, simuladas numericamente, com e sem o efeito de auto-aquecimento.

\subsection{CARACTERÍSTICAS ANALÓGICAS}

A fim de analisar as características analógicas dos transistores, consideraremos o amplificador de tensão baseado em um único transistor em 
configuração de fonte comum e uma carga capacitiva, $\mathrm{C}_{\mathrm{L}}$, apresentado na Figura 10. Neste circuito, o transistor é polarizado por uma fonte de corrente constante ( $\left.I_{\text {bias}}\right)$, responsável por fixar seu ponto de operação, permitindo que a parcela alternada, $v_{\mathrm{gf}}$, da tensão $V_{\mathbb{I N}}$ aplicada à porta seja amplificada, compondo a parcela alternada, $\mathrm{V}_{\mathrm{ds}}$, da tensão de saída $V_{\text {OUT. }}$

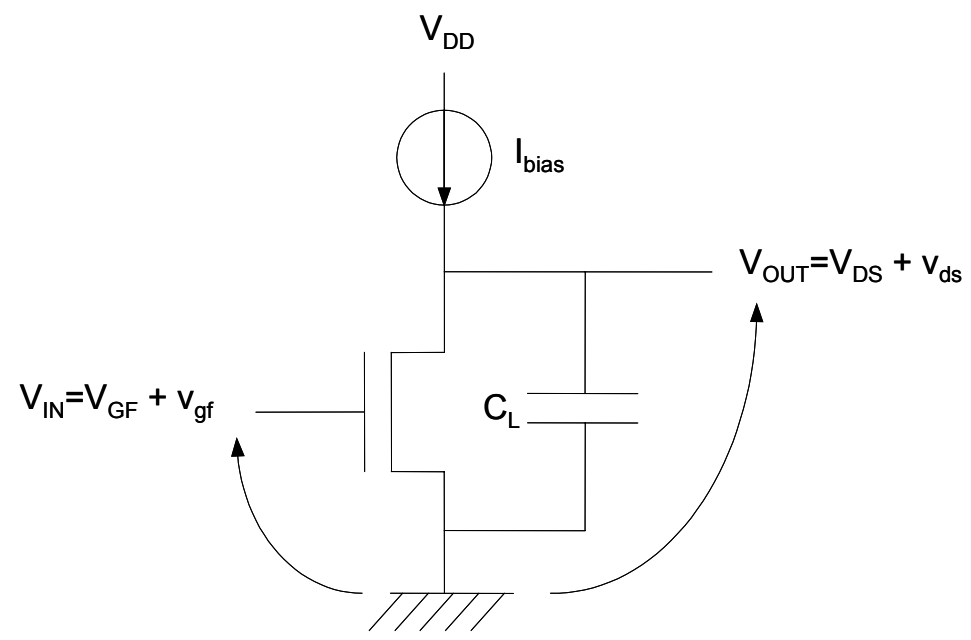

Figura 10 - Amplificador de tensão com um único transistor MOS.

O ganho de tensão de malha aberta em baixas freqüências, $A_{V}$, de um transistor MOS operando como amplificador de tensão é dado por ${ }^{75}$ :

$$
A_{v}=\frac{v_{d s}}{v_{g f}}=\frac{g_{m}}{g_{D}}
$$

o qual pode ser aproximado por

$$
A_{V}=\frac{g_{m}}{g_{D}}=\frac{g_{m}}{I_{D S}}\left|V_{E A}\right|
$$

na região de saturação, onde $g_{D}$ é a condutância de dreno e $V_{E A}$, a tensão Early. $A$ relação $g_{m} / I_{D S}$ e a tensão Early serão apresentadas a seguir. 


\subsubsection{Relação $g_{m} / I_{D S}$}

A relação $g_{m} / I_{D S}$ traduz a eficiência do transistor em converter uma dada corrente de polarização em transcondutância. Seu valor máximo ocorre quando o transistor está operando em inversão fraca e, para transistores MOS convencional e SOI, é dado por ${ }^{76}$ :

$$
\frac{g_{m}}{l_{D S}}=\frac{q}{n k T}
$$

Em inversão forte, há uma redução no valor da relação $g_{m} / I_{D S}{ }^{77}$ :

$$
\frac{g_{m}}{I_{D S}}=\sqrt{\frac{2 \mu_{n} C_{o x f} \frac{W}{L}}{n l_{D S}}}
$$

Devido ao menor fator de corpo, $\mathrm{n}$, em dispositivos SOI totalmente depletados, a relação $\mathrm{g}_{\mathrm{m}} / \mathrm{I}_{\mathrm{DS}}$ é maior em dispositivos SOI MOSFET totalmente depletados que em transistores MOS convencionais. Tipicamente, o valor máximo de $\mathrm{g}_{\mathrm{m}} / \mathrm{I}_{\mathrm{DS}}$ em um transistor SOI MOSFET é de $35 \mathrm{~V}^{-1}$, enquanto em MOSFETs convencionais, atinge cerca de $25 \mathrm{~V}^{-1} 35$.

A Figura 11 apresenta as curvas da relação $g_{m} / l_{D S}$ em função da corrente de dreno normalizada $\left(\mathrm{I}_{\mathrm{DS}} /(\mathrm{W} / \mathrm{L})\right)$, simuladas para transistores SOI totalmente depletados com $L=0,5 \mu \mathrm{m}$, operando em saturação $\left(V_{D S}=1,2 V\right)$, em diferentes temperaturas.

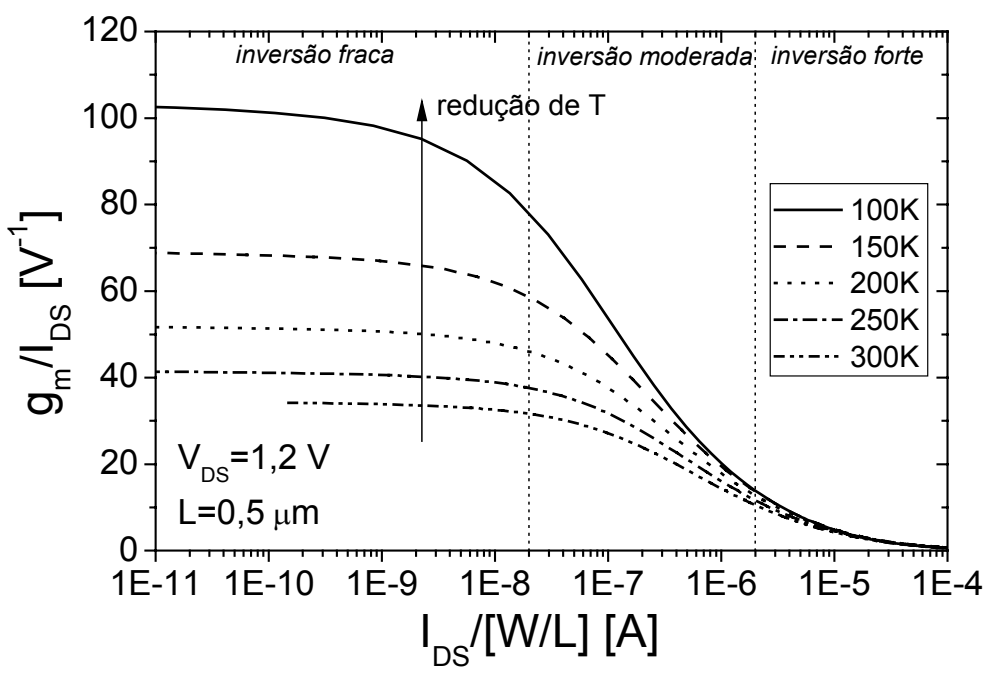

Figura 11 - Relação $g_{m} / I_{D S}$ em função de $I_{D S} /(W / L)$, simulada para transistores SOI totalmente depletados com $L=0,5 \mu \mathrm{m}$, operando em saturação $\left(V_{D S}=1,2 \mathrm{~V}\right)$, em diferentes temperaturas. 
A partir da eq. (45), que descreve a relação $g_{m} / l_{D S}$ em inversão fraca, pode-se notar a dependência direta desta grandeza com o inverso da temperatura. Em inversão forte, a relação $g_{m} / l_{D S}$ é dependente da temperatura, através da raiz quadrada da mobilidade (eq. (46)). Desta forma, pode-se notar que a redução da temperatura provoca o aumento da relação $\mathrm{g}_{\mathrm{m}} / \mathrm{l}_{\mathrm{DS}}$ nos três regimes de inversão (fraca, moderada e forte). Entretanto, em inversão fraca este aumento tende a ser maior devido à dependência direta com a redução da temperatura. Nestas considerações se está admitindo que o fator de corpo seja praticamente independente da temperatura, isto é, as densidades de armadilhas de interface são desprezíveis em baixas temperaturas.

\subsubsection{Tensão Early}

Em um transistor MOS, quando a tensão aplicada ao dreno é maior do que a tensão de saturação, a região de depleção próxima ao dreno aumenta. Como resultado, o comprimento efetivo do canal sofre uma redução, provocando um acréscimo na corrente de dreno na saturação (l $\left.\mathrm{I}_{\mathrm{DSsat}}\right)$. Este acréscimo se traduz em um aumento da inclinação da curva da corrente de dreno ( $\left.I_{D S}\right)$ em função da tensão entre dreno e fonte $\left(V_{D S}\right)$ na região de saturação ${ }^{50}$. Desta forma, a equação da corrente de dreno simplificada, em saturação (eq. (4)), pode ser reescrita, para a inclusão deste efeito ${ }^{78}$ :

$$
\mathrm{I}_{\mathrm{DSsat}} \cong \frac{\mathrm{W} \mu_{\mathrm{n}} \mathrm{C}_{\mathrm{oxf}}}{2 \mathrm{Ln}}\left(\mathrm{V}_{\mathrm{GF}}-\mathrm{V}_{\mathrm{thf}}\right)^{2}\left(1+\lambda \mathrm{V}_{\mathrm{DS}}\right)
$$

onde $\lambda$ é um parâmetro dependente do processo.

Considerando que a tensão Early pode variar com a polarização $V_{D S}$, sua extração é realizada em função de $V_{D S}$, na região de saturação ${ }^{50}$ :

$$
\left|V_{E A}\right| \cong \frac{I_{D S s a t}}{g_{\text {Dsat }}}
$$

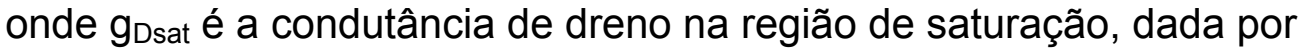




$$
g_{\text {Dsat }}=\frac{\mathrm{dl}_{\text {DSsat }}}{d V_{\text {DS }}} \cong \frac{W_{\mu_{n}} C_{\text {oxf }}}{2 L n}\left(V_{G F}-V_{\text {thf }}\right)^{2} \lambda
$$

Uma vez que a tensão Early é basicamente a mesma em transistores SOI e MOS convencional, observa-se um maior ganho de tensão nos dispositivos SOI totalmente depletados (eq. (44)), devido ao maior valor da relação $\mathrm{g}_{\mathrm{m}} / \mathrm{l}_{\mathrm{DS}}$ destes transistores, considerando dispositivos idênticos, isto é, com mesmas dimensões.

A redução da temperatura, com conseqüente aumento da mobilidade dos portadores, provoca o aumento (ou degradação) da condutância de dreno, como pode ser observado através da eq. (49). Além disso, como discutido anteriormente, em temperaturas reduzidas e com altas polarizações de dreno, o fenômeno de ionização por impacto torna-se mais pronunciado, aumentando a parcela de corrente adicional proveniente da geração de pares elétron-lacuna, causando o aumento da condutância de dreno na região de saturação (e conseqüente redução da tensão Early). Estes dois efeitos podem ser vistos na Figura 12, na qual são apresentadas as curvas da condutância de dreno em função da tensão de dreno, resultantes da derivada de curvas da corrente de dreno em função da tensão de dreno, obtidas a partir de simulações numéricas de transistores SOI totalmente depletados com $L=$ 0,5 $\mu \mathrm{m}$, com sobretensão de condução $\left(\mathrm{V}_{\mathrm{GT}}\right)$ de $200 \mathrm{mV}$, em diferentes temperaturas.

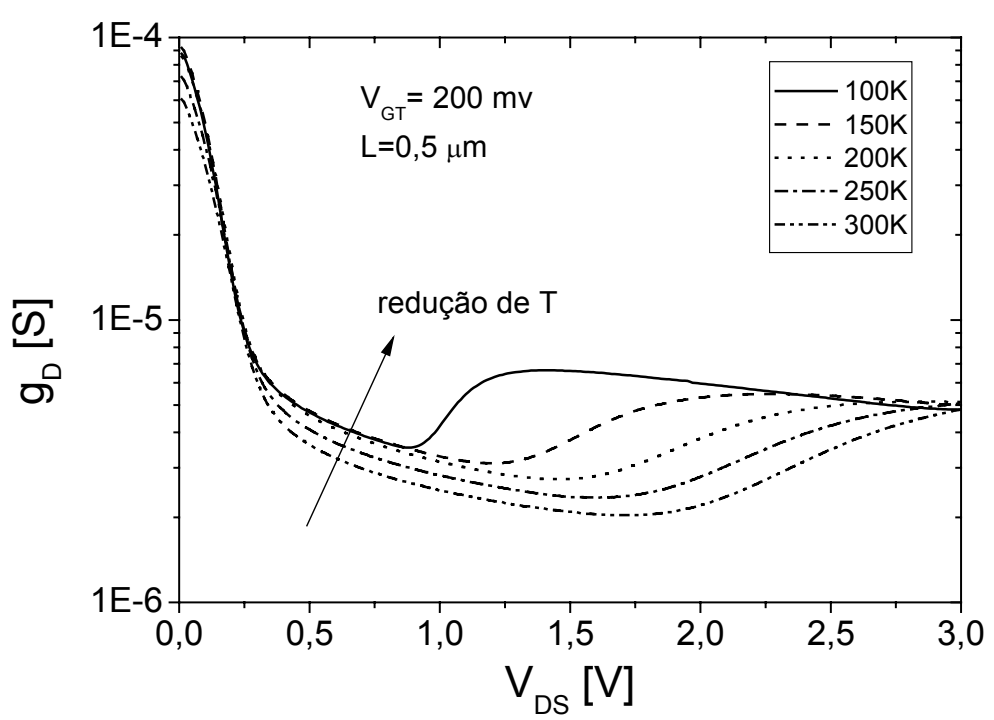

Figura 12 - Condutância de dreno em função da tensão de dreno, simulada para transistores SOI totalmente depletados com $\mathrm{L}=0,5 \mu \mathrm{m}$, com $\mathrm{V}_{\mathrm{GT}}=200 \mathrm{mV}$, em diferentes temperaturas. 


\subsubsection{Distorção Harmônica}

Um dos qualificadores importantes da resposta analógica de um dispositivo é sua linearidade que pode ser quantificada pela distorção harmônica. Aplicando-se um sinal senoidal à porta de um transistor MOS, o que se observa no dreno deste mesmo transistor, quando polarizado com corrente constante, é a presença do sinal fundamental e de inúmeros harmônicos, devidos à natureza não-linear das curvas de corrente do transistor ${ }^{79}$. Para aplicações analógicas, é importante que a quantidade e a amplitude destes harmônicos sejam desprezíveis em relação ao sinal original ${ }^{79}$.

Existem diversas formas para a caracterização da linearidade de um transistor. Neste trabalho, utilizamos a chamada distorção harmônica ${ }^{80}$, adotando como índices de mérito a distorção harmônica total (Total Harmonic Distortion THD) e a distorção do harmônico de terceira ordem (Third Order Harmonic Distortion - HD3). Considerando o sinal de saída, a distorção resultante de cada harmônico corresponde à razão entre a amplitude deste harmônico e a amplitude da componente fundamental, enquanto a distorção harmônica total é dada pela raiz quadrada da soma dos quadrados das amplitudes das componentes de frequências múltiplas à fundamental dividida pelo quadrado da amplitude da componente fundamental ${ }^{82}$.

A distorção harmônica total, em geral, é a figura de mérito de maior importância, apesar de não permitir a distinção entre os harmônicos de ordem par e ímpar. Por outro lado, a distorção do harmônico de terceira ordem, que corresponde ao primeiro harmônico de ordem ímpar presente no sinal de saída, permite comparar o nível de distorção introduzido por harmônicos pares e ímpares. Embora esta componente seja desprezível em relação à THD na maior parte das aplicações, sua obtenção torna-se importante em alguns casos, como por exemplo, em circuitos balanceados que suprimem os harmônicos de ordem par ${ }^{83}$.

De acordo com a ref. [81], considerando o transistor polarizado como amplificador, para baixas amplitudes de sinal senoidal aplicado à sua entrada (Va), THD, que em saturação, é dominado pela distorção do harmônico de segunda ordem (HD2 - Second Order Harmonic Distortion), pode ser expresso por: 


$$
T H D \cong H D 2=\frac{1}{2} V a \frac{\frac{d g_{m}}{d V_{G T}}}{2 g_{m}}
$$

ou ainda

$$
\frac{T H D}{A_{V}} \cong \frac{H D 2}{\frac{g_{m}}{g_{D}}}=\frac{1}{2} V a \frac{\frac{d g_{m}}{d V_{G T}}}{2 g_{m}{ }^{2}} g_{D}
$$

Neste trabalho, as figuras de mérito para a análise da distorção serão extraídas utilizando o Método da Função Integral (Integral Function Method - IFM) ${ }^{82}$. Neste método, as características de distorção são obtidas através de curvas estáticas dos transistores, sem a necessidade da realização de medidas em corrente alternada e de aplicação de métodos baseados na série de Fourier para a obtenção de THD e HD3, os quais são muito mais complexos e sujeitos a componentes parasitas dos dispositivos e dos sistemas de medidas ${ }^{83}$.

\subsection{TRANSISTORES SOI DE CANAL GRADUAL}

Os transistores SOI totalmente depletados apresentam reduzida tensão de ruptura de dreno, devido ao efeito de corpo flutuante. Este efeito é decorrente da presença da estrutura bipolar parasitária intrínseca ao transistor MOS, no qual a fonte é o emissor, o canal é a base e o dreno é o coletor. Elevando-se a tensão aplicada ao dreno, com conseqüente elevação do campo elétrico, as lacunas geradas próximo ao dreno são injetadas na região de fonte fortemente dopada, que é o emissor do transistor bipolar parasitário. Uma vez que a concentração de dopantes da região de fonte é muito maior do que a do canal, uma grande quantidade de elétrons é injetada nesta, resultando em uma corrente de coletor (de dreno) considerável. Desta forma, o efeito bipolar parasitário é influenciado pela quantidade de lacunas geradas pelo mecanismo de ionização por impacto junto ao dreno. Esta concentração de lacunas, por sua vez, é dependente da tensão aplicada 
à porta e inversamente proporcional à concentração de dopantes na região do canal $^{24}$.

Diminuindo-se a concentração de dopantes em um dos lados da junção obtém-se uma redução da barreira de potencial e, conseqüentemente, do campo elétrico. Com um menor campo elétrico, uma menor quantidade de portadores é gerada por ionização por impacto, diminuindo o fator de multiplicação de corrente do transistor bipolar, responsável pela amplificação da corrente de lacunas que é injetada na fonte.

Uma alternativa utilizada para a elevação da tensão de ruptura dos transistores nMOS, convencionais e SOI, é a estrutura com o dreno fracamente dopado (Lightly Doped Drain - LDD) ${ }^{84}$. Nesta estrutura, uma região com concentração reduzida de dopante tipo $n$ entre a região de canal (tipo $p$ ) e a região de dreno (tipo $\mathrm{n}$ ) é criada com o objetivo de diminuir a barreira de potencial da junção, minimizando a geração de portadores por impacto. Entretanto, esta estrutura apresenta, como maior inconveniente, a elevação da resistência série associada ao transistor, já elevada em transistores SOI pela redução da espessura da camada de silício ${ }^{85,86}$. Com o mesmo objetivo, poder-se-ia diminuir a concentração de dopantes do lado p da junção, ou seja, da região de canal. Essa redução, entretanto, provocaria a redução da tensão de limiar do dispositivo e a elevação do ganho de corrente do transistor bipolar parasitário $\left(\beta^{\prime}\right)$, que são efeitos indesejáveis.

A fim de promover a redução da ionização por impacto na região próxima ao dreno, mantendo a tensão de limiar e $\beta^{\prime}$ em valores aceitáveis, foi proposta uma nova estrutura SOI, denominada transistor SOI MOSFET com canal gradual (Graded-Channel SOI MOSFET - GC SOI MOSFET) ${ }^{12}$. Esta estrutura apresenta um perfil de dopantes assimétrico na região do canal, a qual é dividida em duas regiões, como pode ser visto na Figura 13, que apresenta o perfil de um GC SOI nMOSFET. A primeira região, junto à fonte e com comprimento $L_{H D}$, apresenta a concentração usual de dopantes dos transistores $\mathrm{SO}$ totalmente depletados $\left(\mathrm{N}_{\mathrm{AH}}\right)$ e é responsável por fixar a tensão de limiar do dispositivo. A outra região, próxima ao dreno, apresenta uma concentração reduzida de dopantes $\left(\mathrm{N}_{\mathrm{AL}}\right)$, promovendo a redução da ionização por impacto. Esta região, que será doravante denominada fracamente dopada, apresenta tensão de limiar negativa e, em uma primeira aproximação, pode ser entendida como uma extensão da região de dreno para valores positivos de tensão aplicada à porta, reduzindo o comprimento de canal efetivo do dispositivo 
( $L_{\text {eff }}=L-L_{L D}$ onde $L$ é o comprimento de máscara do dispositivo e $L_{L D}$ é o comprimento da região fracamente dopada).

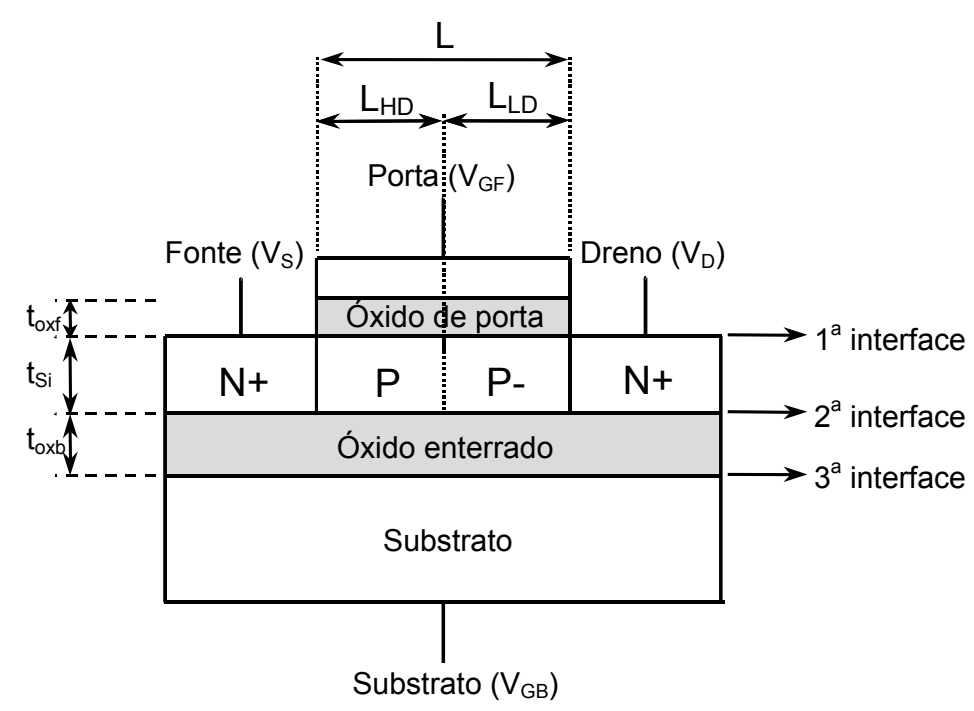

Figura 13 - Perfil do transistor SOI MOSFET canal $n$ totalmente depletado com perfil de dopantes assimétrico na região de canal (GC SOI nMOSFET).

Trabalhos comparativos entre dispositivos GC SOI MOSFET e SOI MOSFET convencionais totalmente depletados ${ }^{88}$ mostram um significativo aumento na corrente de dreno e da transcondutância máxima nos transistores com canal gradual. Tal fato deve-se à redução do comprimento efetivo de canal, que ocorre à medida que a relação $L_{L D} / L$ aumenta.

Além de proporcionar o aumento da corrente de dreno e da transcondutância máxima, a estrutura GC SOI proporciona uma significativa melhora na condutância de dreno e, conseqüentemente, elevação da tensão Early ${ }^{87}$, quando comparada a transistores SOI convencionais, além do aumento da tensão de ruptura de dreno $\left(B V_{D S}\right)^{88}$. A melhora em $g_{D}$ oferecida pela estrutura GC SOI é superior àquela obtida pela associação série de transistores com porta em curto-circuito ${ }^{89}$, que é uma estratégia comum para o aumento de g em circuitos CMOS analógicos ${ }^{90,91,92}$.

Estudos realizados em blocos analógicos básicos, tais como espelhos de corrente e amplificadores operacionais ${ }^{13,93}$ demonstram o enorme potencial dos dispositivos GC SOI para estas aplicações. No caso dos espelhos de corrente, 
obteve-se uma melhora na precisão de espelhamento, aumento de mais de $50 \%$ na excursão de saída ${ }^{13}$ e resistência de saída três vezes maior do que espelhos de corrente implementados com transistores SOI convencionais com as mesmas dimensões ${ }^{94}$. Amplificadores operacionais implementados com transistores de canal gradual apresentaram aumento de mais de $10 \mathrm{~dB}$ no ganho de tensão, sem degradação da margem de fase ${ }^{14}$. Outros trabalhos reportam ainda vantagens da estrutura GC SOI para aplicações em microondas ${ }^{95}$ e rádio-frequência ${ }^{96}$.

As primeiras investigações da operação analógica de transistores GC SOI em baixas temperaturas foram realizadas na ref. [97], na faixa de temperaturas entre $300 \mathrm{~K}$ e $100 \mathrm{~K}$. Neste trabalho, ficou demonstrado que, para esta faixa de temperaturas, os transistores GC SOI apresentam ganho de tensão de malha aberta, $A_{V}, 20 \mathrm{~dB}$ maior do que o transistor convencional. Em um mesmo ponto de polarização, foi observada uma degradação de cerca de $10 \mathrm{~dB}$ em $A_{V}$, quando a temperatura de transistores GC SOI foi reduzida de $300 \mathrm{~K}$ para $100 \mathrm{~K}{ }^{97}$, indicando que a degradação de $\mathrm{V}_{\mathrm{EA}}$ com a temperatura é maior do que o aumento da razão $g_{m} / l_{\text {DS. }}$

\subsubsection{Modelo analítico contínuo para transistores GC SOI}

De acordo com a ref. [98], o transistor GC SOI pode ser interpretado como a associação série de dois transistores SOI uniformemente dopados, com as portas curto-circuitadas, cada um representando uma parte do canal - regiões fortemente (HD) e fracamente (LD) dopadas, como apresentado na Figura 14. Nesta figura, $V_{D S E}$ corresponde à tensão entre o 'dreno virtual', $V_{D, H D}$, e a tensão de fonte do transistor de canal gradual. 


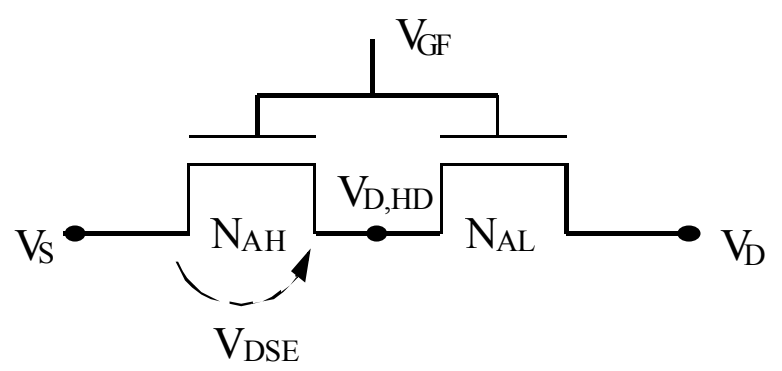

Figura 14 - Associação série de dois transistores SOI convencionais, representando o comportamento elétrico do transistor GC SOI.

Com base nesta consideração, foi desenvolvido um modelo analítico contínuo para a simulação de transistores GC SOI, orientado para aplicações em circuitos analógicos ${ }^{99}$. De acordo com este modelo, a corrente de dreno (IDS) do transistor de canal gradual pode ser obtida considerando-o como um transistor SOI convencional, representado pela região fortemente dopada (que é responsável por fixar as características do dispositivo, como por exemplo, a tensão de limiar), cuja tensão de dreno, $V_{D, H D}$, é uma fração da tensão aplicada ao dreno do dispositivo $\left(V_{D}\right)$, e depende das características das duas regiões do canal.

A expressão de corrente de dreno, incluindo os efeitos de degradação da mobilidade, modulação do comprimento de canal e velocidade de saturação do portador de carga pode ser vista na eq. (52).

$$
\mathrm{I}_{\mathrm{DS}}=\frac{\mathrm{W}}{\mathrm{L}_{\text {eff }}} \frac{\mu_{\mathrm{n}}}{1+\mathrm{V}_{\mathrm{DE}} \frac{\mu_{\mathrm{n}}}{\mathrm{L}_{\text {eff }} \mathrm{v}_{\text {sat }}}}\left[\mathrm{v}_{\mathrm{T}}\left(\mathrm{Q}_{\mathrm{D}, \mathrm{HD}}-\mathrm{Q}_{\mathrm{S}, \mathrm{HD}}\right)-\frac{\mathrm{Q}_{\mathrm{D}, \mathrm{HD}}^{2}-\mathrm{Q}_{\mathrm{S}, \mathrm{HD}}^{2}}{2 \mathrm{nC} \mathrm{C}_{\text {oxf }}}\right]
$$

onde $\mu_{\mathrm{n}}$ é a mobilidade na camada de inversão, dependente do campo elétrico tanto na direção vertical como horizontal, calculada como apresentado anteriormente, $\mathrm{v}_{\mathrm{T}}=\frac{\mathrm{kT}}{\mathrm{q}}$ é o potencial térmico, $\mathrm{L}_{\text {eff }}=\mathrm{L}-\mathrm{L}_{\mathrm{LD}}-\Delta \mathrm{L}-\mathrm{L}_{\text {sat, }} \Delta \mathrm{L}$ sendo a redução do comprimento de canal devida à difusão lateral, $L_{\text {sat }}$ é o comprimento da região saturada, $Q_{D, H D}$ e $Q_{S, H D}$ são as densidades de carga de inversão por unidade de área no dreno e na fonte da região fortemente dopada, expressas por ${ }^{100}$ 


$$
Q_{i, H D}=C_{o x f} n v_{T}\left\{1-\sqrt{1+\frac{4\left[-C_{o x f} n v_{T} S_{N T} \cdot \ln \left(1+\sqrt{\frac{-Q_{0} /\left(2 C_{o x f}\right)}{n v_{T} S_{N T}^{2}}} \times e^{K 1}+e^{k 2}\right)\right.}{\left(C_{o x f} n v_{T}\right)^{2}}}\right\}
$$

onde o índice i é igual a D para a densidade de carga de inversão no dreno, e igual a $S$ na fonte, $S_{N T}(<1)$ é um parâmetro de ajuste que controla a transição entre as regiões de inversão fraca e forte, $K 1=\frac{V_{G F}-V_{\text {thfl }}-n V(y)}{2 n v_{T}}$ e $K 2=\frac{V_{G F}-V_{\text {thf }}-n V(y)}{2 n v_{T} S_{N T}}$, onde $V(y)$ é a tensão no canal, igual a $V_{D E}$ e $V_{S}$, respectivamente, em $y=L-L_{L D} e$ $\mathrm{y}=0, \bigvee_{\text {thf }}$ e $\mathrm{V}_{\text {thfl }}$ são as tensões de limiar equivalentes em inversão forte e fraca, $V_{G F}$ é tensão de porta, $V_{D E}$ é a tensão de dreno efetiva, $V_{S}$ é a tensão de fonte e $Q_{0}$ é a densidade de carga de inversão para $\mathrm{V}_{\mathrm{GF}}=\mathrm{V}_{\text {thfl }} 100$.

$A$ tensão $V_{D S E}$, que corresponde à queda de tensão efetiva entre o dreno da região fortemente dopada do canal e a fonte $\left(V_{D E}-V_{S}\right)$, pode ser calculada através das equações abaixo, propostas na ref. [99].

$$
\mathrm{V}_{\mathrm{DSE}}=\mathrm{V}_{\mathrm{DS}, \mathrm{SAT}}-\mathrm{V}_{\mathrm{DS}, \mathrm{SAT}} \frac{\ln \left[1+\exp \left(\mathrm{A}_{\mathrm{TS}}\left(1-\frac{\mathrm{V}_{\mathrm{D}, \mathrm{HD}}}{\mathrm{V}_{\mathrm{DS}, \mathrm{SAT}}}\right)\right)\right]}{\ln \left[1+\exp \left(\mathrm{A}_{\mathrm{TS}}\right)\right]}
$$

onde $A_{T S}$ é o parâmetro que controla a transição entre as regiões de triodo e saturação, $V_{D S, S A T}$ é a tensão de saturação ${ }^{100}$ e $V_{D, H D}$ é a tensão de dreno da região fortemente dopada do canal, expressa por

$$
\mathrm{V}_{\mathrm{D}, \mathrm{HD}}=\mathrm{V}_{\mathrm{D}, \mathrm{HD}, \mathrm{SAT}}-\mathrm{V}_{\mathrm{D}, \mathrm{HD}, \mathrm{SAT}} \frac{\ln \left[1+\exp \left(\mathrm{B}_{\mathrm{TS}}\left(1-\frac{\mathrm{V}_{\mathrm{D}, \mathrm{HD}, \mathrm{LIN}}}{\mathrm{V}_{\mathrm{D}, \mathrm{HD}, \mathrm{SAT}}}\right)\right)\right]}{\ln \left[1+\exp \left(\mathrm{B}_{\mathrm{TS}}\right)\right]}
$$

onde $\mathrm{B}_{\mathrm{TS}}$ é um parâmetro de ajuste que controla a transição entre as regiões de triodo e saturação e $V_{D, H D, S A T}$ e $V_{D, H D, L I N}$ são as tensões de dreno nestas duas condições de operação, que consideram o efeito da região fracamente dopada do canal. $V_{D, H D, L I N}$ é dado por

$$
V_{D, H D, L I N}=\frac{-B_{T}+\sqrt{B_{T}^{2}-4 A_{T} C_{T}}}{2 A_{T}}
$$


onde

$$
\begin{aligned}
& A_{T}=-\frac{\mu_{n H D} \cdot n}{2 \cdot\left(L-L_{L D}\right)}+\frac{\mu_{n L D}}{L_{L D}}\left(\frac{n}{2}-1\right), B_{T}=\frac{\mu_{n H D} \cdot V_{G T, H D}}{\left(L-L_{L D}\right)}+\frac{\mu_{n L D} \cdot\left[V_{G T, D}+V_{D}(1-n)\right]}{L_{L D}} \\
& e C_{T}=-\frac{\mu_{n L D}}{L_{L D}}\left[V_{G T, L D} \cdot V_{D}-\frac{n_{D}^{2}}{2}\right] ;
\end{aligned}
$$

e $V_{D, H D, S A T}$ é dado por

$$
\mathrm{V}_{\mathrm{D}, \mathrm{HD}, \mathrm{SAT}}=\mathrm{V}_{\text {INTERMSAT }}+\mathrm{V}_{\mathrm{D}, \mathrm{HDO}}
$$

onde

$$
\mathrm{V}_{\text {INTERM }, \mathrm{SAT}}=\frac{1}{\mathrm{n}}\left(\frac{\mathrm{v}_{\text {satLD }}}{\mathrm{v}_{\text {satHD }}} \frac{\mathrm{Q}_{\mathrm{D}, \mathrm{LD}}}{\mathrm{C}_{\mathrm{oxf}}}+\mathrm{V}_{\mathrm{GT}, \mathrm{HD}}\right)
$$

e

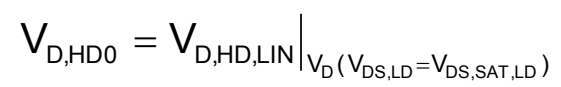

sendo $V_{D}\left(V_{D S, L D}=V_{D S, S A T, L D}\right)=\frac{-B_{S}+\sqrt{B_{S}{ }^{2}-4 A_{S} C_{S}}}{2 A_{S}}$ com

$$
\begin{aligned}
& A_{S}=\frac{\mu_{n L D}}{2 v_{s a t, L D} v_{T} S_{N T}} \frac{\mu_{n H D} V_{G T, H D} L_{L D}+S_{N T} \mu_{n L D} V_{G T, L D}\left(L-L_{L D}\right)}{\left.S_{N T} v_{T} V_{G T, H D} L_{L D}+S_{N T} \mu_{n L D} V_{G T, L D}\left(L-L_{L D}\right)\right]} \\
& B_{S}=\frac{\mu_{n H D} V_{G T, H D} L_{L D}+S_{N T} \mu_{n L D} V_{G T, L D}\left(L-L_{L D}\right)}{S_{N T} v_{T}\left[\mu_{n H D} V_{G T, H D} L_{L D}+S_{N T} \mu_{n L D} V_{G T, L D}\left(L-L_{L D}\right)\right]} e \\
& C_{S}=-\log \left(\frac{-Q_{0} / C_{o x f}}{n v_{T} S_{N T}}\right)-\frac{V_{G F}-V_{\text {thfi,LD }}}{n v_{T}} .
\end{aligned}
$$

Nas expressões acima, $V_{D}$ é a tensão de dreno aplicada à estrutura GC SOI, $V_{\mathrm{GT}}=\mathrm{V}_{\mathrm{GF}}-\mathrm{V}_{\text {thfj }}$ é a sobretensão de condução, $\mathrm{V}_{\text {thfj }}$ é a tensão de limiar em inversão forte (onde $\mathrm{j}=\mathrm{HD}$ para a região fortemente dopada e $\mathrm{j}=\mathrm{LD}$ para a região fracamente dopada do canal), e $\bigvee_{\text {thfix }}$ é a tensão de limiar equivalente em inversão fraca.

Os resultados obtidos através do modelo proposto foram confrontados com dados experimentais e de simulações numéricas bidimensionais, em temperatura ambiente, apresentando uma excelente concordância, com erro inferior a $7 \%$, validando as equações propostas ${ }^{99}$. 


\subsection{SIMULAÇÃO NUMÉRICA BIDIMENSIONAL EM FUNÇÃO DA TEMPERATURA}

Ao longo deste trabalho serão apresentados resultados de simulações numéricas bidimensionais, seja com o intuito de validar equações propostas ou auxiliar o entendimento de resultados obtidos experimentalmente. Para estas simulações, foi utilizado o simulador de dispositivos Atlas ${ }^{101}$.

Este programa permite discretizar dispositivos eletrônicos através de uma grade de pontos e, através da solução das equações de Poisson e de continuidade de corrente, entre outras, possibilita a simulação numérica do comportamento elétrico de dispositivos e pequenos circuitos. Além disso, o simulador possui uma série de modelos físicos que permitem a inclusão de efeitos decorrentes da redução da temperatura sobre as características elétricas dos semicondutores.

Neste trabalho, as simulações foram realizadas desde a temperatura ambiente, $300 \mathrm{~K}$, até $90 \mathrm{~K}$. Foram incluídos nas simulações, os seguintes modelos:

- BGN: considera o estreitamento da largura da faixa proibida;

- INCOMPLETE: considera a ionização incompleta das impurezas, conforme discutido na seção 2.2.2;

- IONIZ: usado para a ionização completa para silício altamente dopado;

- CONSRH: dependência do tempo de vida dos portadores com a concentração de dopantes;

- AUGER: recombinação Auger;

- KLAASSEN: considera a dependência da mobilidade inicial com a temperatura e concentração de dopantes; este modelo inclui os mecanismos de espalhamento de rede, por impurezas ionizadas e portador-portador e por impurezas neutras, discutidos anteriormente, na seção 2.3.4.

- WATT: calcula a mobilidade considerando os principais mecanismos de espalhamento na camada de inversão: espalhamento resultante da interação entre os portadores da camada de inversão e fônons do substrato e devido à rugosidade superficial, ambos dependentes do campo elétrico transversal (seção 2.3.4.(f)).

- MOD.WATT: extensão do modelo WATT para nós não-superficiais; 
- FLDMOB: este modelo inclui o cálculo da mobilidade dependente do campo elétrico lateral, considerando o efeito de redução da mobilidade devido à velocidade de saturação, conforme descrito na seção 2.3.4.1.

- IMPACT.SELB: indica que a geração de portadores por ionização por impacto é incluída na solução da simulação.

Em alguns casos, parâmetros dos modelos de mobilidade foram ajustados, a fim de obter resultados mais próximos dos experimentais. 


\section{MODELAGEM DA CORRENTE DE DRENO DE TRANSISTORES GC SOI E SUAS DERIVADAS}

Neste capítulo será apresentada a adequação do modelo analítico para a corrente de dreno de transistores GC SOI a fim de permitir a simulação de circuitos operando em baixas temperaturas. Será apresentado ainda o desenvolvimento de equações analíticas para a transcondutância e condutância de dreno destes transistores.

\subsection{ADEQUAÇÃO DO MODELO ANALÍTICO CONTÍNUO PARA OPERAÇÃO EM BAIXAS TEMPERATURAS}

O modelo analítico para transistores GC SOI, apresentado na seção 2.5.1, foi inicialmente proposto para a operação em temperatura ambiente. Para temperaturas abaixo de $300 \mathrm{~K}$, este modelo é incapaz de reproduzir com precisão as características elétricas dos transistores GC SOI, uma vez que o potencial $V_{D E}$ depende das mobilidades, velocidade de saturação e tensões de limiar das duas regiões do canal que, como discutido anteriormente, são parâmetros dependentes da temperatura.

Desta forma, diferentes modelos devem ser utilizados, a fim de considerar os fenômenos físicos específicos à operação em baixas temperaturas. Em particular no modelo de GC SOI, as alterações provocadas pela temperatura, estudadas previamente, foram incorporadas ao modelo analítico, utilizando os modelos discutidos no capítulo anterior ${ }^{102}$.

O cálculo da mobilidade inicial, devido aos mecanismos de espalhamento apresentados (eq. (32)), bem como da mobilidade sob a influência do campo elétrico (eq. (33)) foram incluídos nas equações do modelo analítico. Além disso, a resistência série associada às regiões de fonte e dreno e às suas extensões, foi incluída no cálculo da corrente, bem como a dependência da velocidade de saturação com a temperatura (eq. (35)) e a ionização incompleta de portadores em todas as regiões de semicondutores (eqs. (10) e (11)). A corrente de dreno causada 
por ionização por impacto, $\mathrm{I}_{\mathrm{ii}}$, também foi considerada, através do modelo proposto na ref. [67].

\subsubsection{Validação do modelo através da comparação com dados experimentais}

Inicialmente, o modelo analítico para transistores SOI de canal gradual adequado para a operação em baixas temperaturas foi implementado no programa

Matlab ${ }^{103}$ e seus resultados, comparados à curvas experimentais de transistores GC SOI. Para esta comparação, foram utilizados transistores GC SOI fabricados no Laboratório de Microeletrônica da Université catholique de Louvain (UCL) - situada na cidade de Louvain-la-Neuve, Bélgica.

Os dispositivos foram fabricados em lâminas SOI Unibond® ${ }^{104}$ com concentração de inicial de dopantes de $10^{15} \mathrm{~cm}^{-3}$, espessura de óxido enterrado de 390 $\mathrm{nm}$ e espessura de óxido de porta $\left(\mathrm{t}_{\mathrm{oxf}}\right)$ de $30 \mathrm{~nm}$, em uma camada de silício com espessura final $\left(t_{S_{i}}\right)$ de $80 \mathrm{~nm}$. A implantação iônica para ajuste da tensão de limiar realizada resulta em uma concentração de dopantes de cerca de $1 \times 10^{17} \mathrm{~cm}^{-3}$. Foram utilizados, nas comparações, dispositivos com largura de canal (W) de $18 \mu \mathrm{m}$, comprimento de canal $(L)$ de $0,5 \mu \mathrm{m}^{102}$, e diferentes relações $L_{L D} / L: 0,18 ; 0,28$ e 0,47, que correspondem a comprimentos de canal efetivos de 0,41;0,36 e 0,26 $\mu \mathrm{m}$, respectivamente. Dispositivos SOI convencional fortemente e fracamente dopados, isto é, com concentrações uniformes de dopantes iguais a $1 \times 10^{17} \mathrm{~cm}^{-3}$ e $1 \times 10^{15} \mathrm{~cm}^{-3} \mathrm{na}$ região de canal, respectivamente, também foram caracterizados, a fim de comparar seu desempenho ao dos transistores com canal gradual e auxiliar na extração de parâmetros.

Os dispositivos tiveram sua temperatura controlada através do equipamento Variable Temperature Micro Probe System, modelo K20 da MMR Technologies e as curvas de corrente foram extraídas utilizando o analisador de parâmetros de semicondutores Agilent 4156C e tempo de integração médio. 
O modelo proposto foi usado para obter as curvas características de transistores GC SOI com as mesmas dimensões e parâmetros tecnológicos dos dispositivos medidos. Estimativas iniciais para os parâmetros do modelo foram obtidas como descrito na ref. [99].

Uma vez que o modelo analítico de transistores GC SOI considera-o como a associação série de dois transistores SOI convencionais, alguns dos parâmetros podem ser obtidos a partir de dispositivos convencionais com concentração de dopantes $\mathrm{N}_{\mathrm{AH}} \mathrm{e}$ $\mathrm{N}_{\mathrm{AL}}$. As tensões de limiar em inversão forte, $\mathrm{V}_{\text {thf,HD }}$ e $\mathrm{V}_{\mathrm{thf}, \mathrm{LD}}$, foram determinadas a partir do ponto máximo da segunda derivada das curvas $I_{D S}$ em função de $V_{G F}$ com baixa tensão aplicada ao dreno ${ }^{105}$ de transistores com canal uniformemente dopado. As tensões de limiar em inversão fraca, $V_{\text {thfi,HD }}$ e $V_{\text {thfi,LD }}$ foram extrapoladas como $V_{\text {thfi }} \cong V_{\text {thf }}$ $-2 v_{T}{ }^{106}$. O fator de corpo, $n$, foi extraído a partir do inverso da inclinação da curva $I_{D S}$ em função de $\mathrm{V}_{\mathrm{GF}}$, medida com baixa tensão de dreno, na região de sublimiar.

Os valores para as mobilidades iniciais das duas regiões do canal do transistor GC SOI, $\mu_{H D}$ e $\mu_{\mathrm{LD}}$, foram calculadas utilizando as equações apresentadas na seção 2.3.4. As constantes de espalhamento, $\alpha_{S, H D}$ e $\alpha_{S, L D}$, responsáveis pela degradação da mobilidade devido ao campo elétrico podem ser encontradas a partir da curva $I_{D S} / \sqrt{g_{m}}$ em função da tensão aplicada à porta, na região de triodo ${ }^{1}$. O parâmetro $\sigma$, que modela o efeito de DIBL, pode ser determinado extraindo-se a tensão de limiar com diferentes valores de $\mathrm{V}_{\mathrm{DS}}$.

Após a determinação dos parâmetros anteriores, os parâmetros $A_{T S}$ e $B_{T S}$ podem ser determinados empiricamente, através do ajuste da transição entre as regiões de triodo e saturação. De forma semelhante, o parâmetro $S_{N T}$ pode ser encontrado ajustando-se a transição entre os regimes de inversão fraca e forte. A Tabela 1 apresenta os parâmetros utilizados para a comparação das curvas de transistores com $L_{L D} / L=0,28$ e 0,47, em 300 e $100 \mathrm{~K}$. 
Tabela 1 - Parâmetros de modelo utilizados na comparação com curvas experimentais obtidas para transistores GC SOI com $L_{L D} / L=0,28$ e 0,47 em 300 e $100 \mathrm{~K}$.

\begin{tabular}{cccccc}
\hline \multirow{2}{*}{ Parâmetro } & \multicolumn{2}{c}{ GC SOI LLD/L=0,28 } & \multicolumn{2}{c}{ GC SOI LLD/L=0,47 } & \multirow{2}{*}{ Unidade } \\
\cline { 2 - 5 } & $300 \mathrm{~K}$ & $100 \mathrm{~K}$ & $300 \mathrm{~K}$ & $100 \mathrm{~K}$ & \\
\hline $\mathrm{V}_{\text {thf,HD }}$ & 0,63 & 0,94 & 0,55 & 0,88 & $\mathrm{~V}$ \\
\hline $\mathrm{V}_{\text {thf,LD }}$ & $-0,31$ & $-0,18$ & $-0,31$ & $-0,18$ & $\mathrm{~V}$ \\
\hline$\mu_{\text {HD }}$ & 805,27 & 2937,52 & 805,27 & 2937,52 & $\mathrm{~cm}^{2} / \mathrm{V} \cdot \mathrm{s}$ \\
\hline$\mu_{\mathrm{LD}}$ & 1380,32 & 11674,76 & 1380,32 & 11674,76 & $\mathrm{~cm}^{2} / \mathrm{V} \cdot \mathrm{s}$ \\
\hline$\alpha_{\mathrm{HD}}$ & $5,0 \times 10^{-7}$ & $6,5 \times 10^{-7}$ & $6,0 \times 10^{-7}$ & $7,0 \times 10^{-7}$ & $\mathrm{~cm} / \mathrm{V}$ \\
\hline$\alpha_{\mathrm{LD}}$ & $5,0 \times 10^{-5}$ & $5,0 \times 10^{-5}$ & $5,0 \times 10^{-5}$ & $5,0 \times 10^{-5}$ & $\mathrm{~cm} / \mathrm{V}$ \\
\hline $\mathrm{S}_{\mathrm{NT}}$ & 0,99 & 0,99 & 0,99 & 0,99 & - \\
\hline $\mathrm{A}_{\mathrm{TS}}$ & 0,80 & 0,80 & 0,80 & 0,80 & - \\
\hline $\mathrm{B}_{\mathrm{TS}}$ & 4,00 & 4,00 & 4,00 & 1,50 & - \\
\hline $\mathrm{I}_{\mathrm{c}}$ & $1,5 \times 10^{-7}$ & $1,5 \times 10^{-7}$ & $1,5 \times 10^{-7}$ & $1,5 \times 10^{-7}$ & $\mathrm{~cm}$ \\
\hline$\sigma$ & $1,0 \times 10^{-2}$ & $1,0 \times 10^{-2}$ & $1,3 \times 10^{-2}$ & $1,3 \times 10^{-2}$ & - \\
\hline$\sigma_{n n n n n}$ & & & & &
\end{tabular}

Foram comparadas curvas de corrente de dreno sob diversas condições de polarização, apresentando um bom ajuste entre os dados experimentais e de modelo, em toda a faixa de temperatura estudada.

A Figura 15 apresenta as curvas de $I_{D S}$ e $g_{m}$ em função $V_{G F}$ para um dispositivo SOI convencional e diferentes transistores GC SOI polarizados com $V_{D S}=0,85 \mathrm{~V}$, operando em $100 \mathrm{~K}$. A partir destas curvas, pode-se notar que o modelo analítico proposto para operação em baixas temperaturas é capaz de descrever o aumento do nível de corrente resultante da redução do comprimento de canal nos transistores GC SOI.

$\mathrm{Na}$ Figura 16 são apresentadas as curvas $\mathrm{I}_{\mathrm{DS}}$ em função de $\mathrm{V}_{\mathrm{GF}}$ para 0 dispositivo com $L_{L D} / L=0,28$, medidas e modeladas em temperaturas entre $100 \mathrm{~K} \mathrm{e}$ 300 K. São também apresentadas as curvas da transcondutância, $g_{\mathrm{m}}$, obtidas através da derivada numérica das curvas de corrente. Como esperado, pode-se observar o aumento do nível de corrente e da transcondutância em inversão forte 
com a redução da temperatura, promovida pelo aumento da mobilidade dos portadores.

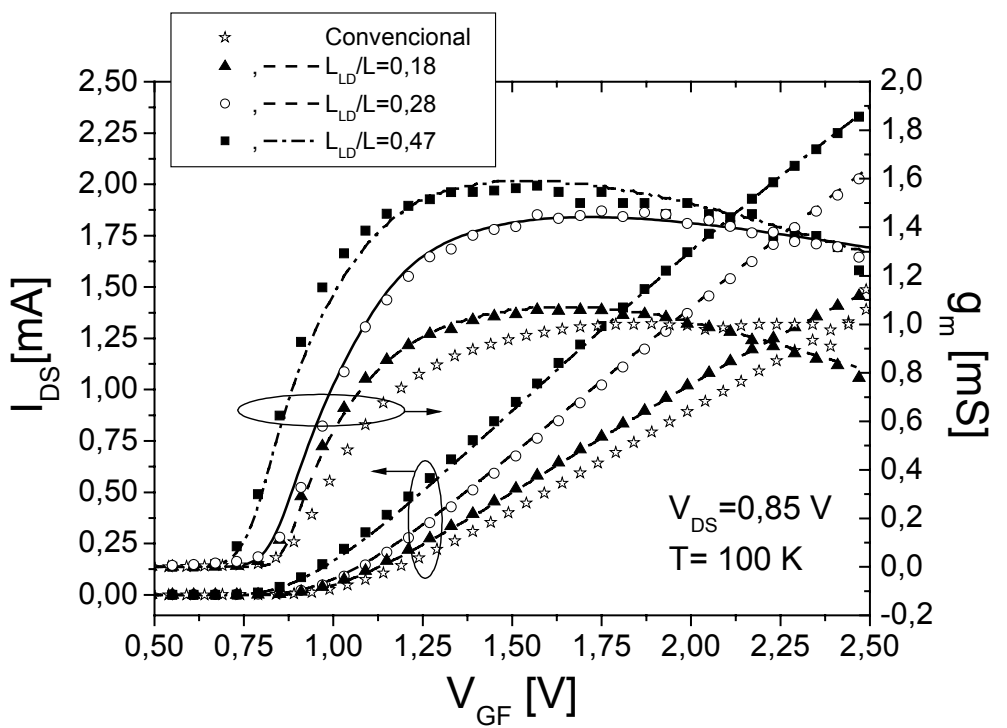

Figura 15 - Comparação entre as curvas da corrente de dreno e transcondutância em função de $V_{G F}$, modeladas (linhas) e medidas (símbolos), para todos os dispositivos operando em $\mathrm{T}=100 \mathrm{~K}$.

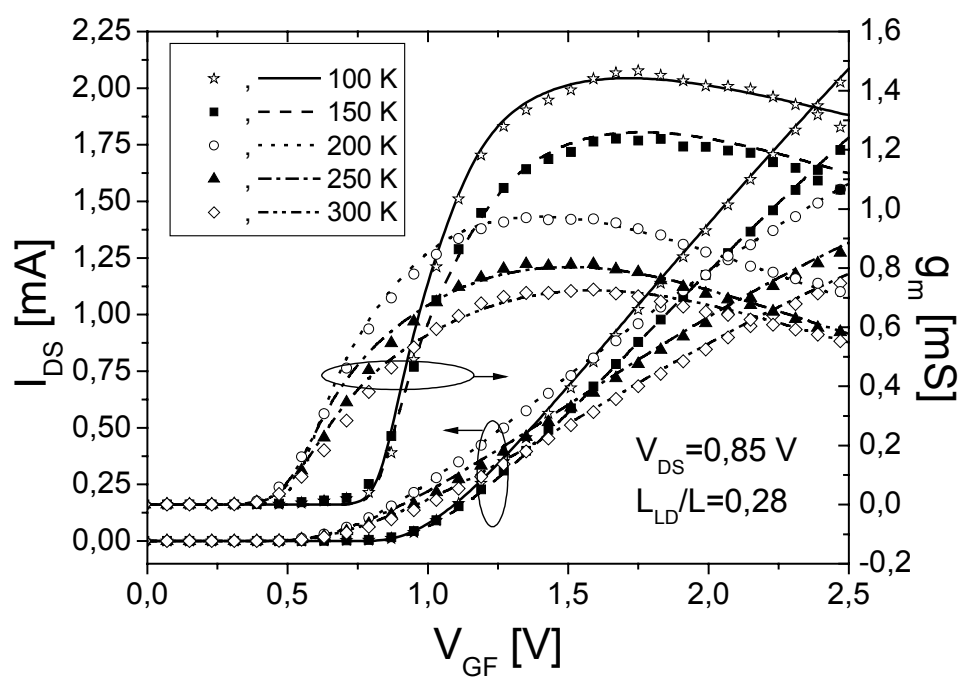

Figura 16 - Curvas da corrente de dreno e transcondutância em função de $V_{G F}$, modeladas (linhas) e medidas (símbolos), em diferentes temperaturas para o dispositivo GC SOI com $L_{L D} / L=0,28$.

Curvas comparativas da corrente e da condutância de dreno em função da tensão de dreno, para diversas temperaturas são apresentadas na Figura 17. A partir das curvas de corrente, pode-se notar que o modelo de ionização por impacto 
utilizado é incapaz de descrever o comportamento da corrente para temperaturas inferiores a $200 \mathrm{~K}$, onde se torna mais significativo ${ }^{107}$. Entretanto, este não é um limitante para a utilização do modelo, uma vez que a região onde ocorre a ionização por impacto não é de interesse para a operação de circuitos analógicos. Desconsiderando esta região, pode-se verificar uma excelente concordância entre os resultados modelados e medidos em todas as temperaturas analisadas. Tomando como referência as curvas experimentais, o erro máximo obtido na comparação entre as curvas $I_{D S}$ em função de $V_{D S}$, em saturação, excetuando-se a região onde o efeito de ionização por impacto é significativo, foi inferior a $4 \%$.
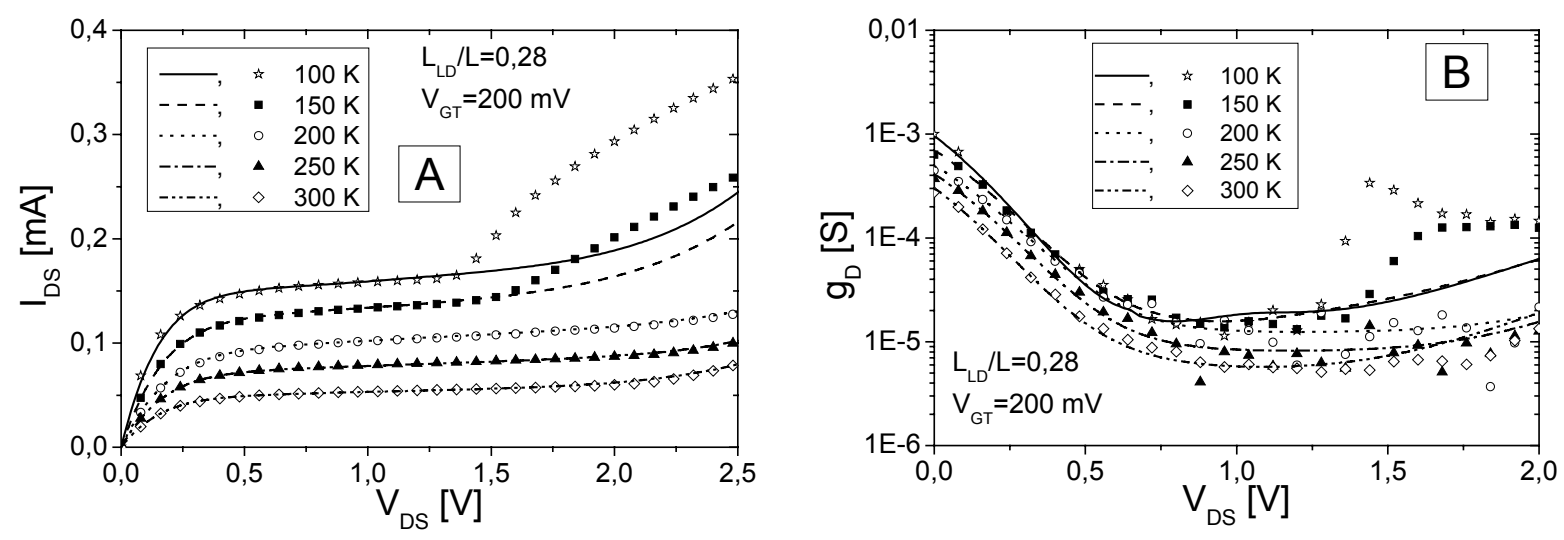

Figura 17 - Curvas $I_{D S}$ em função de $V_{D S}(A)$ e g em função de $V_{D S}(B)$, para dispositivos GC SOI com $L_{L D} / L=0,28$ medidas (símbolos) e modeladas (linhas) em diversas temperaturas com $\mathrm{V}_{\mathrm{GT}}=200 \mathrm{mV}$.

A fim de testar a validade do modelo em diferentes níveis de inversão, foram traçadas as curvas $I_{D S}$ em função de $V_{D S}$ de dispositivos GC SOI com $L_{L D} / L=0,47$ em $300 \mathrm{~K}$ (Figura 18), e $100 \mathrm{~K}$ (Figura 19), variando $\mathrm{V}_{\mathrm{GT}}$ de 0 a $600 \mathrm{mV}$. No caso das curvas em $100 \mathrm{~K}$, o efeito de ionização por impacto foi desprezado na obtenção das curvas modeladas. Nas mesmas figuras são apresentadas as curvas da condutância de dreno, obtidas através da diferenciação numérica das curvas de corrente, apresentadas em escala logarítmica. A partir destas curvas pode-se verificar a continuidade da transição entre os regimes de operação linear e de saturação, que é bastante importante para a simulação de circuitos analógicos. A comparação entre as curvas da corrente de dreno modeladas e medidas em função da tensão de dreno, em temperatura ambiente, resultou em erros máximos inferiores a $2 \% \mathrm{e}$, menores que $3 \%$ em $100 \mathrm{~K}$. 

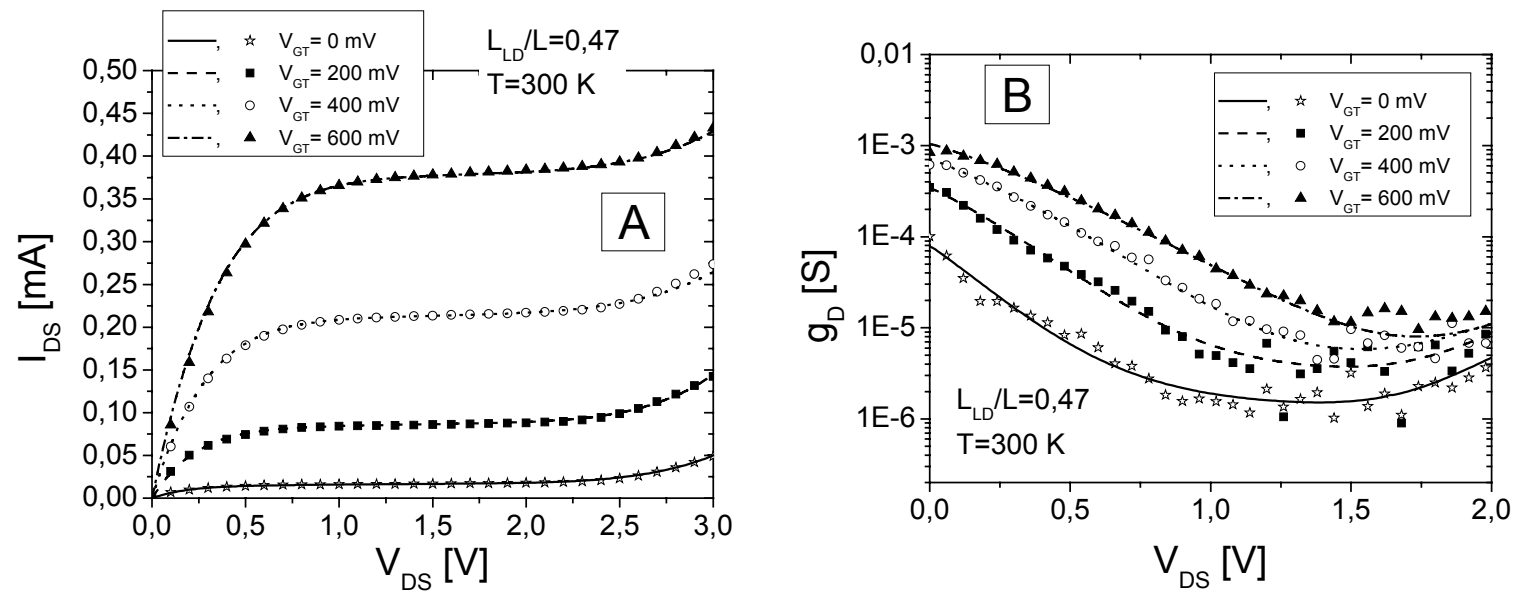

Figura 18 - Curvas $I_{D S}$ em função de $V_{D S}(A)$ e $g_{D}$ em função de $V_{D S}(B)$, para dispositivos GC SOI com $L_{L D} / L=0,47$ medidas (símbolos) e modeladas (linhas) sob diversas condições de polarização de dreno, com $\mathrm{T}=300 \mathrm{~K}$.
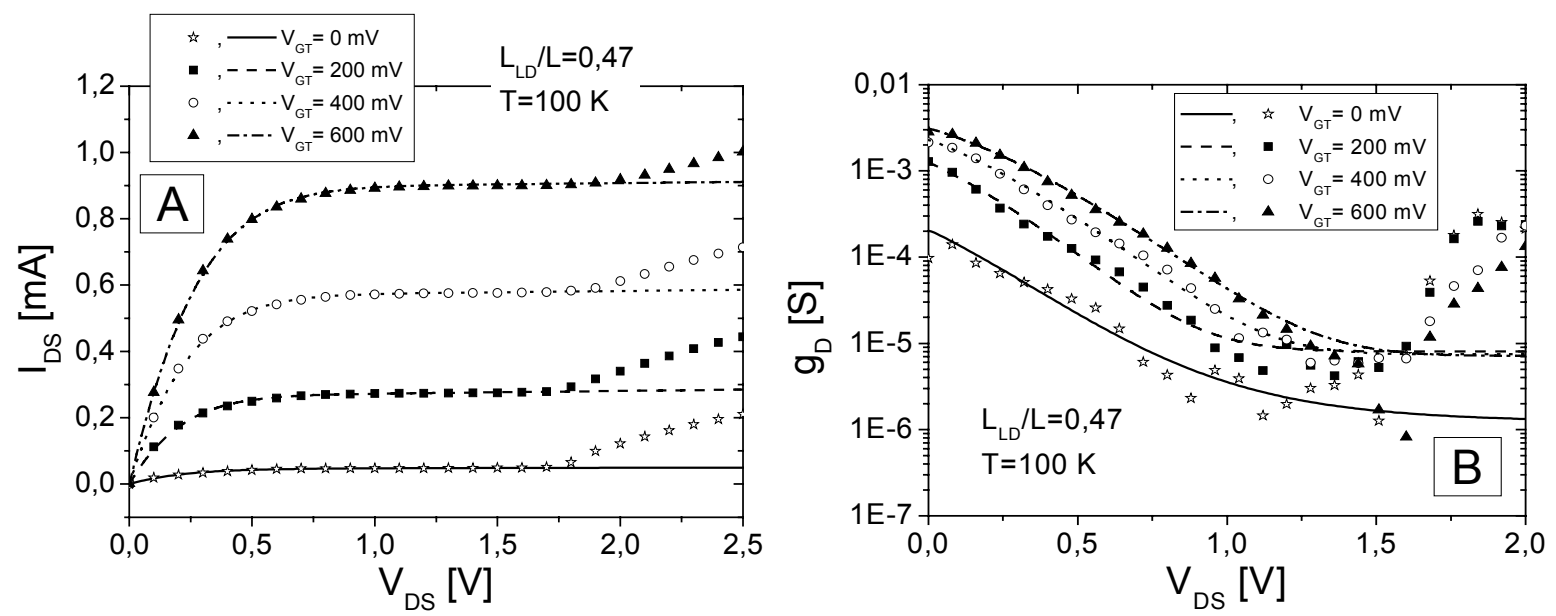

Figura 19 - Curvas $I_{D S}$ em função de $V_{D S}(A)$ e gD em função de $V_{D S}(B)$, para dispositivos GC SOI com $L_{L D} / L=0,47$ medidas (símbolos) e modeladas (linhas) sob diversas condições de polarização de dreno, com T=100 K.

A partir das curvas de $g_{m}$ e $g_{D}$, obtidas experimentalmente e pela adequação do modelo analítico de corrente de transistores GC SOI para baixas temperaturas, foram obtidos os valores do ganho intrínseco, $A_{V}$, em todas as temperaturas estudadas, para o dispositivo com $L_{L D} / L=0,28$, polarizado com $V_{D S}=0,85 \mathrm{~V}$ e $\mathrm{V}_{\mathrm{GT}}=200 \mathrm{mV}$. Os resultados são apresentados na Tabela 2. A partir dos resultados, pode-se observar que o ganho deste dispositivo tende a reduzir com a redução da temperatura, indicando que o aumento da relação $g_{m} / l_{D S}$ com a redução da 
temperatura não é suficiente para compensar a redução da tensão Early, provocada pela degradação da condutância de dreno.

Tabela 2 - Ganho intrínseco obtido através do modelo analítico e de dados experimentais, para os dispositivos $\mathrm{GC}$ com $\mathrm{L}_{\mathrm{LD}} / \mathrm{L}=0,28$, polarizados com $\mathrm{V}_{\mathrm{GT}}=200 \mathrm{mV}$ e $\mathrm{V}_{\mathrm{DS}}=0,85 \mathrm{~V}$.

\begin{tabular}{|c|c|c|c|c|c|c|}
\hline \multicolumn{2}{|c|}{ Temperatura [K] } & 100 & 150 & 200 & 250 & 300 \\
\hline \multirow{2}{*}{$\begin{array}{c}\text { Ganho } \\
\text { intrínseco } \\
{[\mathrm{dB}]}\end{array}$} & Experimental & 19,66 & 19,37 & 33,59 & 35,36 & 37,37 \\
\hline & Modelo & 19,25 & 19,23 & 33,69 & 35,34 & 37,47 \\
\hline
\end{tabular}

\subsection{INCLUSÃO DO MODELO ANALÍTICO EM UM SIMULADOR DE CIRCUITOS}

Com o intuito de viabilizar simulações de células analógicas com dispositivos de canal gradual, é necessária a inclusão de seu modelo analítico em um simulador de circuitos. Assim, após a validação do modelo analítico de transistores GC SOI em baixas temperaturas, este foi incluído na biblioteca de modelos do simulador Eldo ${ }^{108}$, utilizando o programa Eldo UDM (User Definable Models).

O simulador Eldo foi escolhido para esta implementação pois, além de ser um simulador de circuitos robusto, permite ao usuário incluir seu próprio modelo, na forma de funções descritas em linguagem C. Estas funções estão localizadas em módulos específicos dos arquivos-fonte fornecidos, onde o usuário deve definir a lista de parâmetros do novo modelo, as equações para o cálculo de corrente, bem como valores para os parâmetros e geometrias que devem ser usados no caso em que estes não forem definidos no arquivo de simulação. Após a implementação, o modelo é compilado e vinculado à biblioteca de modelos do simulador. 
Foi também incluído no simulador, além do modelo transistor GC SOI, o modelo para transistores SOI totalmente depletados ${ }^{100}$, tipo $\mathrm{n}$ e tipo $\mathrm{p}$, a fim de permitir a simulação de circuitos incluindo também estes transistores.

\subsubsection{Validação da implementação através da comparação com dados simulados}

A fim de validar a implementação do modelo analítico contínuo da corrente de dreno de transistores GC SOI no simulador de circuitos, foram obtidas curvas características através de simulações numéricas bidimensionais, realizadas com o simulador Atlas.

Foram simulados dispositivos GC SOI com $L=0,5 \mu \mathrm{m}$ e relações $L_{L D} / L=0,2$; 0,4 e 0,5, entre $100 \mathrm{~K}$ e $300 \mathrm{~K}$. Também foi simulado um dispositivo SOI com canal uniformemente dopado, com concentração igual à da região fortemente dopada, para comparação, e um transistor SOI com canal fracamente dopado, para auxiliar na extração de parâmetros do modelo. As demais características dos dispositivos simulados são: $t_{\mathrm{oxf}}=15 \mathrm{~nm}, \mathrm{t}_{\mathrm{Si}}=50 \mathrm{~nm}, \mathrm{t}_{\mathrm{oxb}}=390 \mathrm{~nm}, \mathrm{~N}_{\mathrm{AH}}=1,2 \times 10^{17} \mathrm{~cm}^{-3}$ e $\mathrm{N}_{\mathrm{AL}}=$ $1 \times 10^{15} \mathrm{~cm}^{-3}$. Todos os dispositivos foram simulados com regiões de LDD (lightly doped drain) com comprimento de $0,1 \mu \mathrm{m}$ e concentração de dopantes de $2 \times 10^{18}$ $\mathrm{cm}^{-3}{ }^{109}$. O Apêndice A apresenta um exemplo de arquivo utilizado para a simulação numérica.

Os parâmetros de modelo implementado no Eldo foram extraídos como descrito na seção 3.1.1, e são apresentados na Tabela 3, para um dispositivo convencional e um GC SOI, em T=300 K e $100 \mathrm{~K}$. 
Tabela 3 - Parâmetros de modelo utilizados na comparação com curvas obtidas através do simulador numérico para transistores convencional e GC SOI com $L_{L D} / L=0,50$, em $300 \mathrm{~K}$ e $100 \mathrm{~K}$.

\begin{tabular}{cccccc}
\hline \multirow{2}{*}{ Parâmetro } & \multicolumn{2}{c}{ Convencional } & \multicolumn{2}{c}{ GC SOI LLD $/ \mathrm{L}=0,50$} & \multirow{2}{*}{ Unidade } \\
\cline { 2 - 5 } & $300 \mathrm{~K}$ & $100 \mathrm{~K}$ & $300 \mathrm{~K}$ & $100 \mathrm{~K}$ & \\
\hline $\mathrm{V}_{\mathrm{thf}, \mathrm{HD}}$ & 0,30 & 0,38 & 0,24 & 0,33 & $\mathrm{~V}$ \\
\hline $\mathrm{V}_{\mathrm{thf}, \mathrm{LD}}$ & - & - & $-0,31$ & $-0,07$ & $\mathrm{~V}$ \\
\hline$\mu_{\mathrm{HD}}$ & 764,20 & 2702,16 & 764,20 & 2702,16 & \\
\hline$\mu_{\mathrm{LD}}$ & - & - & 1380,32 & 11674,75 & \\
\hline$\alpha_{\mathrm{HD}}$ & $1,0 \times 10^{-7}$ & $3,6 \times 10^{-6}$ & $1,0 \times 10^{-7}$ & $3,7 \times 10^{-6}$ & $\mathrm{~cm} / \mathrm{V}$ \\
\hline$\alpha_{\mathrm{LD}}$ & - & - & $1,5 \times 10^{-7}$ & $2,5 \times 10^{-7}$ & $\mathrm{~cm} / \mathrm{V}$ \\
\hline $\mathrm{V}_{\mathrm{sat}, \mathrm{HD}}$ & $1,0 \times 10^{7}$ & $2,6 \times 10^{7}$ & $1,0 \times 10^{7}$ & $2,6 \times 10^{7}$ & $\mathrm{~cm} / \mathrm{s}$ \\
\hline $\mathrm{v}_{\mathrm{sat}, \mathrm{LD}}$ & - & - & $1,0 \times 10^{7}$ & $2,6 \times 10^{7}$ & $\mathrm{~cm} / \mathrm{s}$ \\
\hline $\mathrm{n}$ & 1,15 & 1,15 & 1,20 & 1,20 & - \\
\hline $\mathrm{S}_{\mathrm{NT}}$ & 0,99 & 0,99 & 0,98 & 0,99 & - \\
\hline $\mathrm{A}_{\mathrm{TS}}$ & 4,50 & 4,50 & 4,00 & 4,00 & - \\
\hline $\mathrm{B}_{\mathrm{TS}}$ & - & - & 4,00 & 4,00 & - \\
\hline $\mathrm{I}_{\mathrm{c}}$ & $1,5 \times 10^{-6}$ & $1,5 \times 10^{-6}$ & $3,0 \times 10^{-6}$ & $3,0 \times 10^{-6}$ & $\mathrm{~cm}$ \\
\hline$\sigma$ & $1,0 \times 10^{-2}$ & $1,0 \times 10^{-2}$ & $3,0 \times 10^{-2}$ & $3,0 \times 10^{-2}$ & - \\
\hline
\end{tabular}

A Figura 20 apresenta as curvas $I_{D S}$ em função de $V_{G F}$, obtidas para os transistores SOI convencional (A) e GC SOI com relação $L_{L D} / L=0,5$ (B) , com $\mathrm{V}_{\mathrm{DS}}=100 \mathrm{mV}$, para diversas temperaturas. Em todas as curvas apresentadas nesta seção, os símbolos representam os resultados das simulações Atlas e as linhas representam os dados obtidos através do simulador de circuitos Eldo. No Apêndice B é apresentado um arquivo de entrada utilizado neste simulador. A Figura 21 apresenta as curvas da transcondutância, obtida através da diferenciação numérica das curvas apresentadas na Figura 20, em função de $V_{G F}$. No detalhe destas figuras são apresentadas as curvas da transcondutância em escala logarítimica, mostrando que o ajuste entre os resultados dos dois simuladores também é bom em inversão fraca. Além disso, pode-se notar que os resultados dos dois simuladores apresentam 
excelente concordância em todas as temperaturas estudadas, com erro inferior a $8 \%$ na comparação entre as curvas da corrente de dreno.
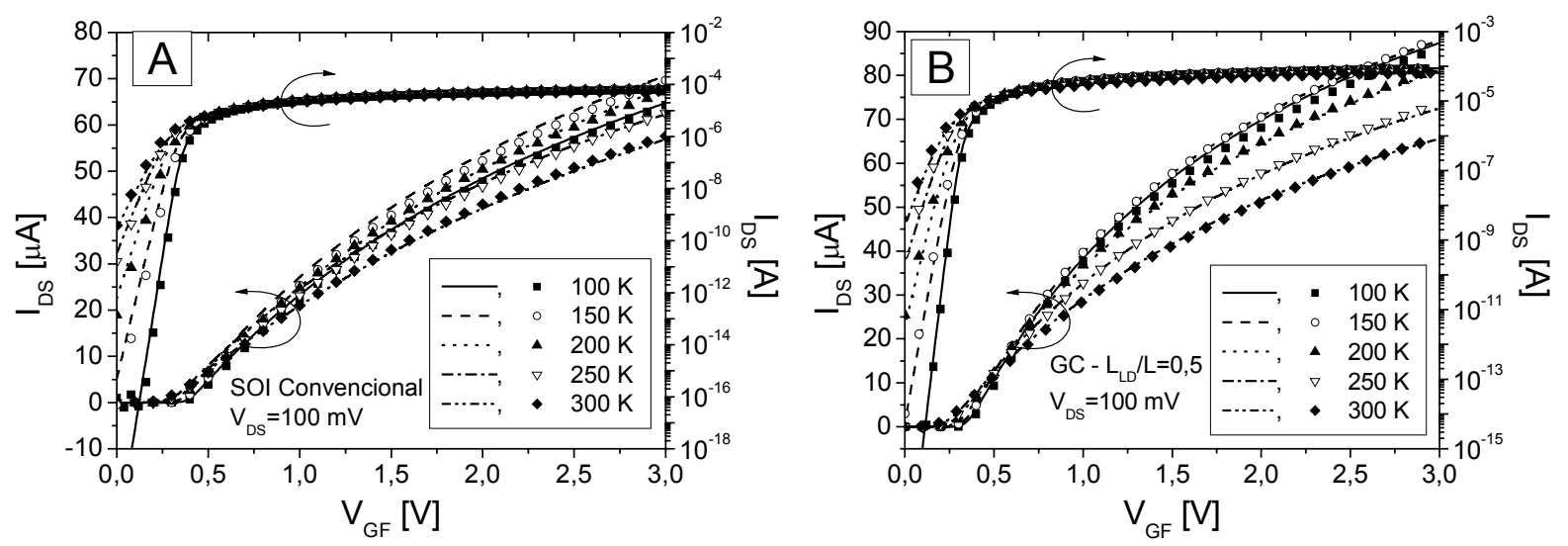

Figura 20 - Comparação entre as curvas $I_{D S}$ em função de $V_{G F}$, com $V_{D S}=100 \mathrm{mV}$, obtidas com os simuladores Eldo (linhas) e Atlas (símbolos), de dispositivos SOI convencional (A) e GC SOI com $L_{L D} / L=0,5(B)$.
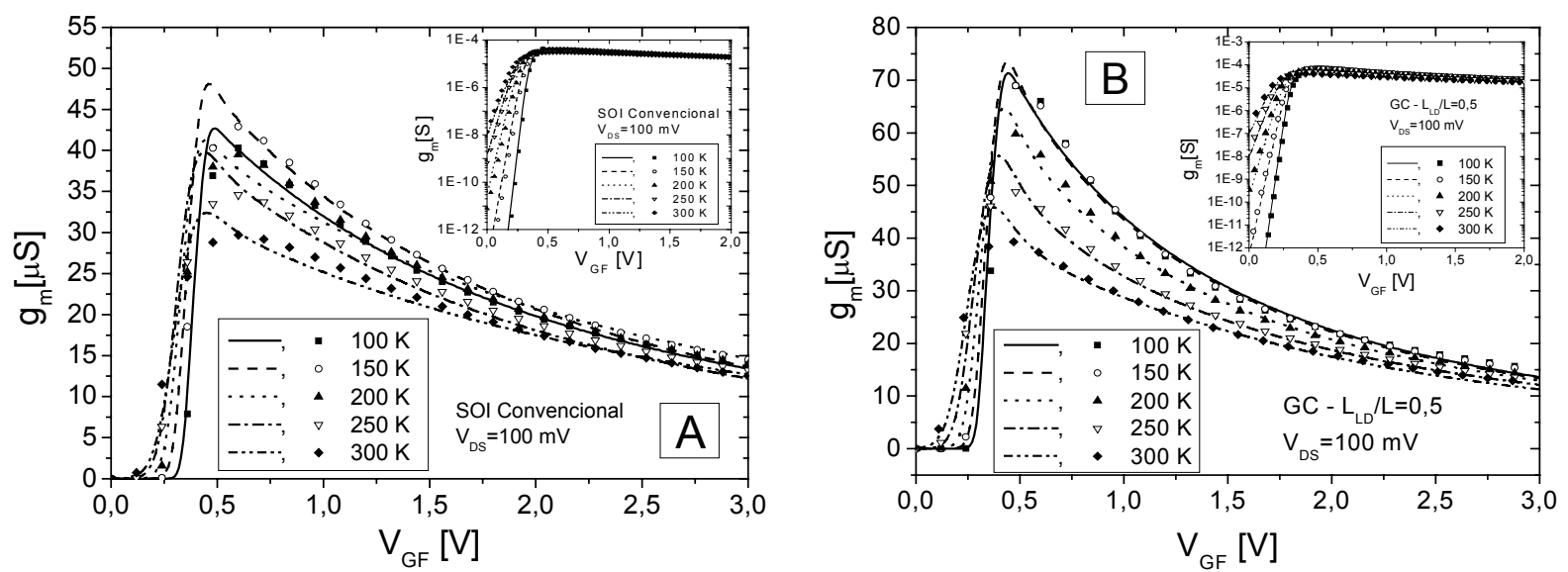

Figura 21 - Comparação entre as curvas da transcondutância em função de $V_{\mathrm{GF}}$, com $V_{D S}=100 \mathrm{mV}$, obtidas com os simuladores Eldo (linhas) e Atlas (símbolos), para dispositivos SOI convencional (A) e GC SOI com $L_{L D} / L=0,5$ (B).

Também para os outros dispositivos foi obtida uma excelente concordância entre os resultados dos dois simuladores. A Figura 22 apresenta as curvas IDs em função de $V_{G F}$ e $g_{m}$ em função de $V_{G F}$, com $V_{D S}=100 \mathrm{mV}$, obtidas com os simuladores Eldo e Atlas, para todos os dispositivos estudados, com T=100 K. Em todos os casos, o erro máximo não excedeu $10 \%$. 


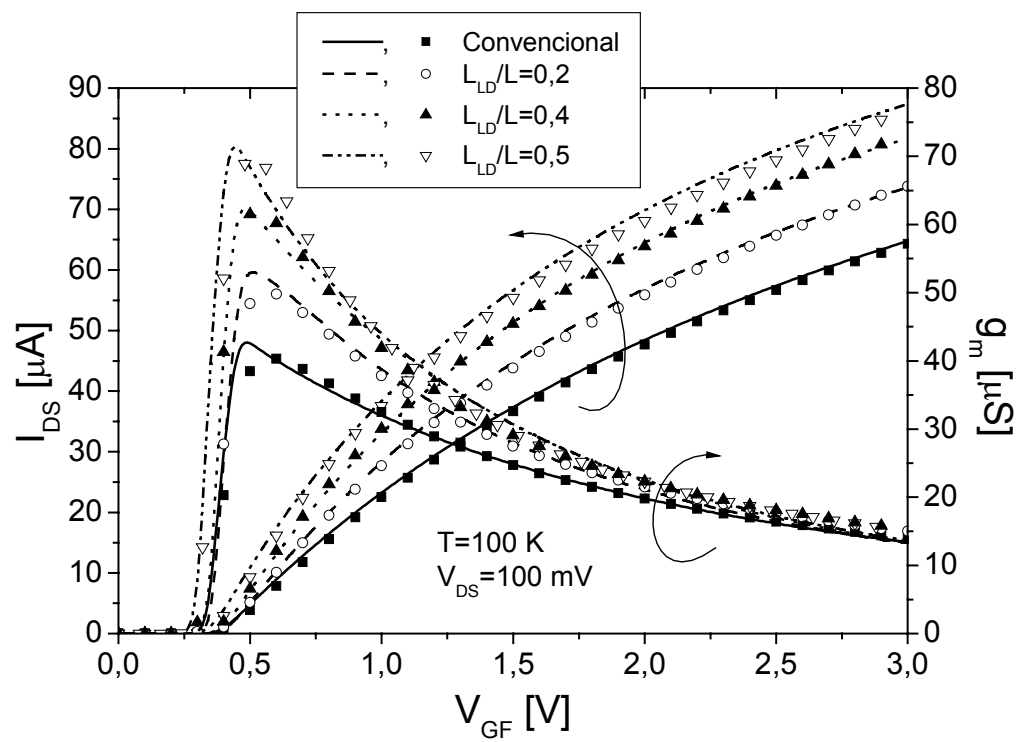

Figura 22 - Comparação entre as curvas $I_{D s}$ e $g_{m}$ em função de $V_{G F}$, com $V_{D S}=100$ $\mathrm{mV}$, obtidas com os simuladores Eldo (linhas) e Atlas (símbolos), para todos os dispositivos estudados, com $\mathrm{T}=100 \mathrm{~K}$.

Foram também comparadas as curvas $I_{D S}$ em função de $V_{G F}$ com $V_{D S}=1,2 \mathrm{~V}$. Os resultados obtidos para o transistor $\mathrm{GC} S \mathrm{SOI}$ com $\mathrm{L}_{\mathrm{LD}} / \mathrm{L}=0,5$, são apresentados na Figura 23. A partir destas curvas, foram obtidas as relações $g_{m} / l_{D S}$ em todas as temperaturas estudadas. As curvas resultantes são apresentadas na Figura 24.

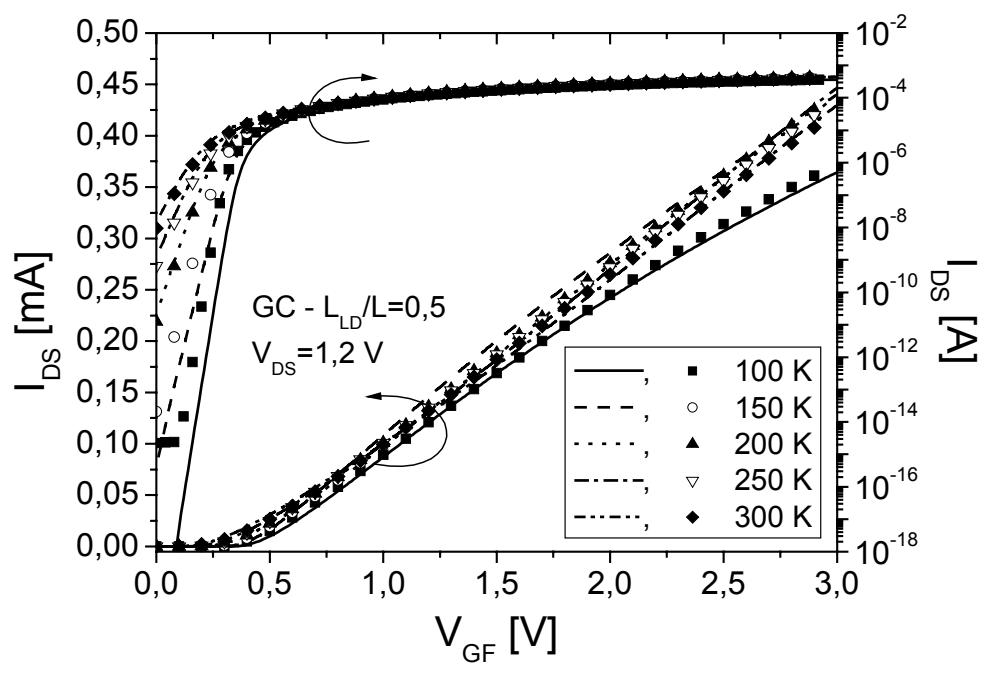

Figura 23 - Comparação entre as curvas $I_{D S}$ em função de $V_{G F}$ com $V_{D S}=1,2 V$, obtidas com os simuladores Eldo (linhas) e Atlas (símbolos), para dispositivos GC $\mathrm{SOI}$ com $L_{L D} / L=0,5$ em diversas temperaturas. 


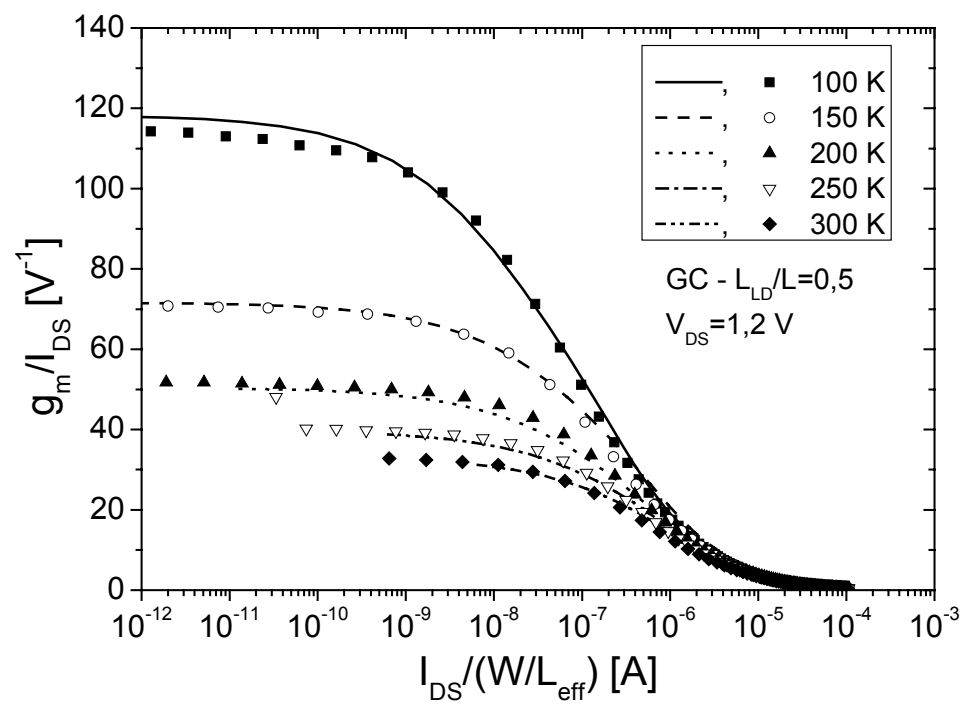

Figura 24 - Comparação entre as curvas $g_{m} / I_{D S}$ em função de $I_{D S} /\left(W / L_{e f f}\right)$, com $\mathrm{V}_{\mathrm{DS}}=1,2 \mathrm{~V}$, obtidas com os simuladores Eldo (linhas) e Atlas (símbolos), para dispositivos GC SOI com $L_{L D} / L=0,5$ em diversas temperaturas.

Como pode ser visto a partir das curvas $I_{D S}$ em função de $V_{D S}$ apresentadas na Figura 25, o modelo consegue descrever, com precisão o comportamento de um transistor GC SOI em diferentes temperaturas de operação, exceto na região onde a ionização por impacto se torna significativa ( $\mathrm{V}_{\mathrm{DS}}>2 \mathrm{~V}$, nestas curvas), uma vez que este efeito não foi incluído na implementação. O mesmo comportamento pode ser visto através da Figura 26, onde são apresentadas as curvas $I_{D S}$ em função de $V_{D S}$ para todos os dispositivos simulados, operando em T=100 K. A partir destas curvas, pode-se notar o aumento da tensão de saturação à medida que o comprimento da região fracamente dopada é aumentado. Na mesma figura são apresentadas as curvas da condutância de dreno, obtidas através da diferenciação numérica das curvas de corrente. Em todos os casos, tomando como referência a corrente de dreno obtida através do simulador numérico, o erro foi inferior a $6 \%$. 


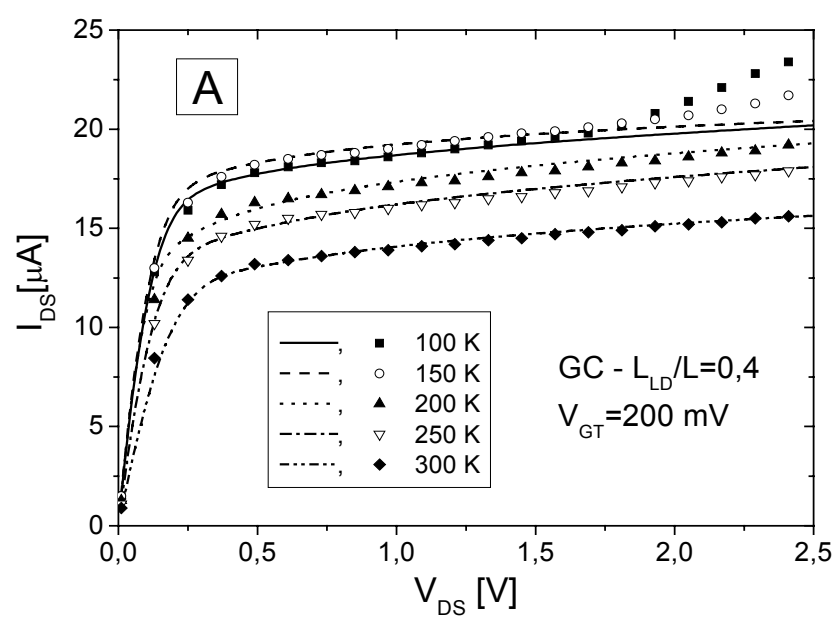

Figura 25 - Comparação entre as curvas $I_{D s}$ em função de $V_{D S}$, com $V_{G T}=200 \mathrm{mV}$, obtidas com os simuladores Eldo (linhas) e Atlas (símbolos), de dispositivos e GC

SOI com $L_{L D} / L=0,4$ em diversas temperaturas.
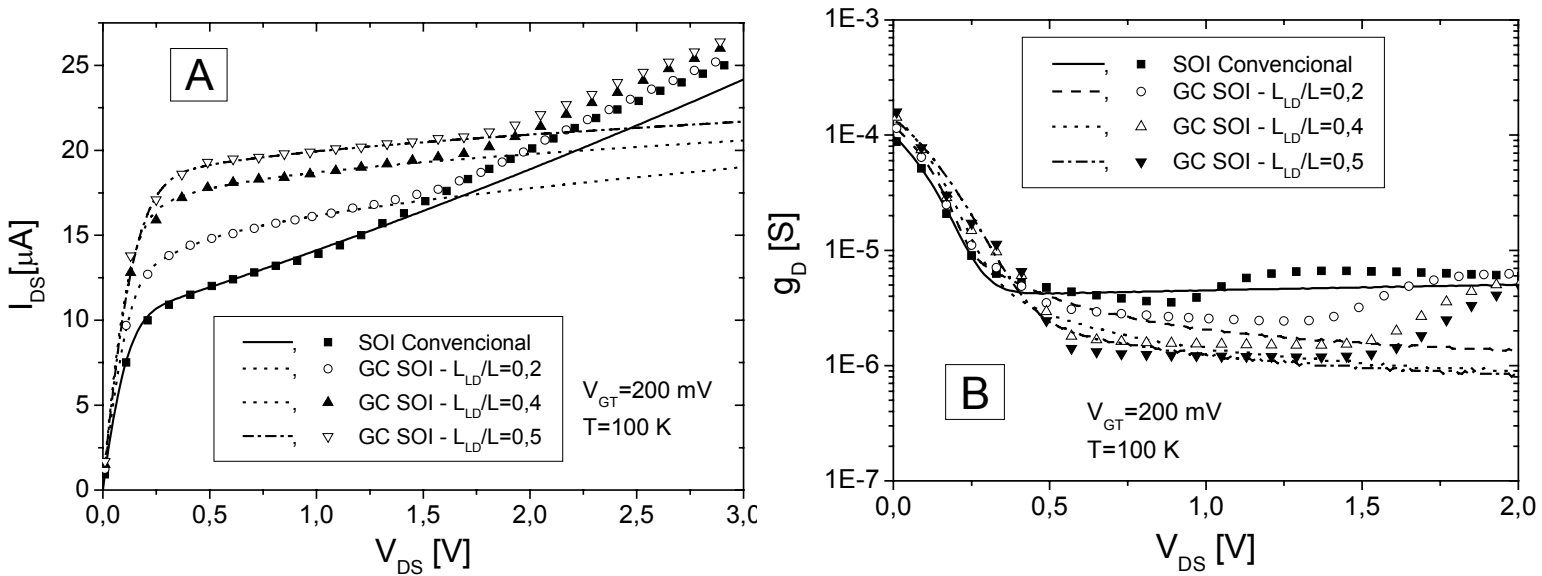

Figura 26 - Comparação entre as curvas $I_{D S}$ e g $g_{D}$ em função de $V_{D S}$, com $V_{G T}=200$ $\mathrm{mV}$, obtidas com os simuladores Eldo (linhas) e Atlas (símbolos), para todos os dispositivos simulados, com $\mathrm{T}=100 \mathrm{~K}$.

A partir dos resultados obtidos, pode-se concluir que o modelo analítico contínuo para transistores GC SOI operando em baixas temperaturas foi implementado com sucesso no simulador de circuitos Eldo. Nas comparações realizadas com resultados de simulações numéricas bidimensionais, o erro máximo no cálculo da corrente de dreno foi inferior a 10\%. Desta forma, o simulador está apto a permitir a simulação de células analógicas com dispositivos de canal gradual operando desde a temperatura ambiente até baixas temperaturas.

SOUZA, M. de; PAVANELLO, M.A.; FLANDRE, D.; Submicrometer Graded-Channel SOI MOSFET Modeling for Low Temperature Analog Circuit Design. Proc. of the $7^{\text {th }}$ International Workshop on Low Temperature Electronics (WOLTE 7), p. 57-64, 2006. 


\subsection{EQUAÇÕES ANALÍTICAS PARA TRANSCONDUTÂNCIA E CONDUTÂNCIA DE DRENO DE TRANSISTORES GC SOI}

A transcondutância $\left(g_{m}\right)$ e a condutância de dreno $\left(g_{D}\right)$ de transistores MOS são parâmetros importantes do ponto de vista de projeto de circuitos analógicos. Desta forma, propusemos expressões explícitas contínuas para estes parâmetros, visando sua utilização como ferramenta de projeto de circuitos ${ }^{110,111}$.

De acordo com a ref. [100], após a inclusão de efeitos de canal curto no modelo analítico contínuo de transistores SOI, não é possível a obtenção de expressões analíticas simples e suficientemente precisas para a transcondutância e condutância de dreno e a diferenciação numérica da curva de corrente passa a ser mais conveniente. Obtivemos, então, expressões analíticas para a transcondutância e condutância de dreno de transistores GC SOI, através de sua definição matemática, diferenciando, analiticamente, a equação de corrente do transistor (dada pela eq. (52)), em relação à $\mathrm{V}_{\mathrm{GF}}$ ou $\mathrm{V}_{\mathrm{D}}$.

\subsubsection{Transcondutância}

Pode-se verificar, numericamente, que o termo $\left(1+\mathrm{V}_{\mathrm{DE}} \frac{\mu_{\mathrm{n}}}{\mathrm{L}_{\mathrm{eff}} \mathrm{V}_{\mathrm{sat}}}\right)^{-1}$ da equação da corrente de dreno (eq. (52)), tem pouca influência sobre a derivada do termo $\left(\frac{W}{L_{\text {eff }}} \frac{\mu_{n}}{1+V_{D E} \frac{\mu_{n}}{L_{\text {eff }} V_{\text {sat }}}}\right)$ em relação a $V_{G F}$, quando a tensão de dreno é mantida constante. Desta forma, sua derivada em relação à $\mathrm{V}_{\mathrm{GF}}$ pode ser desprezada. Como resultado, a transcondutância de um transistor GC pode ser obtida através da eq. (60), que é dependente das densidades de carga de inversão e de suas derivadas. Nesta equação, a densidade de carga de inversão $Q_{D, H D}$ por unidade de área, bem como sua derivada, incluem o efeito da estrutura GC sobre a tensão de dreno efetiva. 


$$
\begin{aligned}
g_{m} & =\frac{d l_{D S}}{d V_{G F}}=\frac{W}{L_{\text {eff }}} \frac{\mu_{n}}{1+V_{D E} \frac{\mu_{n}}{L_{\text {eff }} v_{\text {sat }}}}\left[V_{T}\left(\frac{d Q_{D, H D}}{d V_{G F}}-\frac{d Q_{S, H D}}{d V_{G F}}\right)-\frac{Q_{D, H D} \frac{d Q_{D, H D}}{d V_{G F}}-Q_{S, H D} \frac{d Q_{S, H D}}{d V_{G F}}}{n C_{\text {oxf }}}\right] \\
& +\frac{\frac{d \mu_{n}}{d V_{G F}}}{1+V_{D E} \frac{\mu_{n}}{L_{\text {eff }} V_{\text {sat }}}} \frac{W}{L_{\text {eff }}}\left[V_{T}\left(Q_{D, H D}-Q_{S, H D}\right)-\frac{Q_{D, H D}^{2}-Q_{S, H D}^{2}}{2 n C_{\text {oxf }}}\right]
\end{aligned}
$$

onde

$$
\frac{d \mu_{n}}{d V_{G F}}=\frac{\mu_{0} \alpha_{S} A}{\left(1+\alpha E_{\text {eff }}\right)^{2}}
$$

$\operatorname{com} A=\frac{d_{S, H D}}{d V_{G F}}\left(\frac{2 \varepsilon_{S i}+t_{S i} n C_{o x f}}{4 \varepsilon_{S i} t_{S i} n C_{o x f}}\right)+\frac{d Q_{D, H D}}{d V_{G F}}\left(\frac{1}{4 \varepsilon_{S i}}\right)$

A derivada da densidade de carga de inversão, por sua vez, é dada por

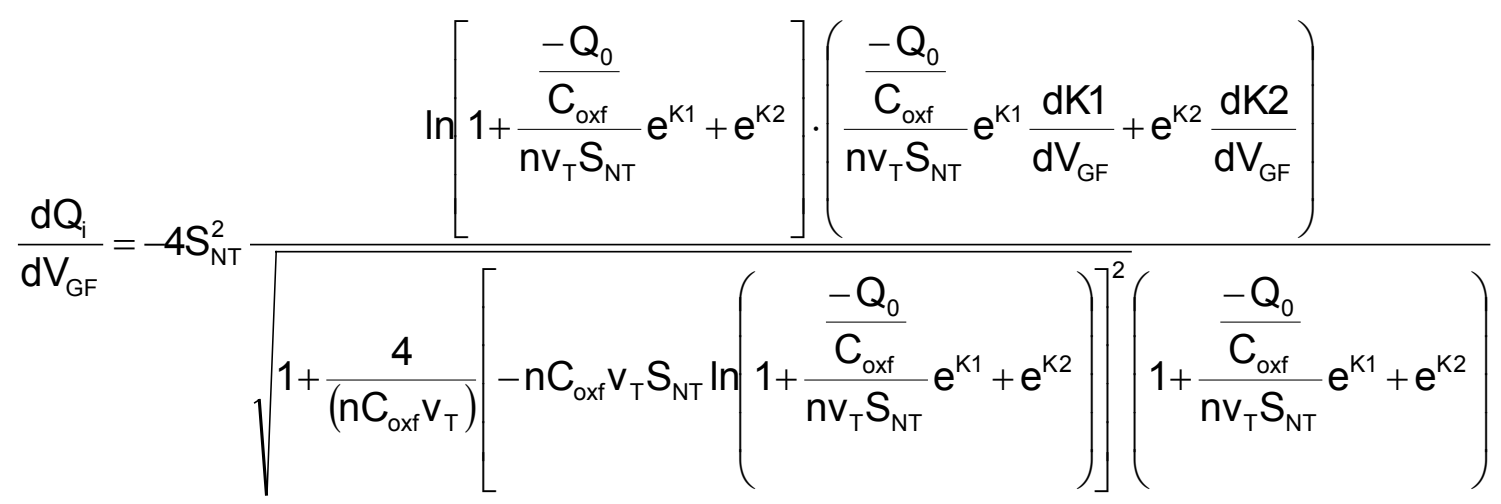

Neste caso, o termo $\frac{\mathrm{dQ}_{\mathrm{S}, \mathrm{HD}}}{\mathrm{dV}_{\mathrm{GF}}}$ é calculado com $\frac{\mathrm{dK} 1}{\mathrm{dV}_{\mathrm{GF}}}=\frac{1}{2 n v_{T}}$ e $\frac{d K 2}{d V_{G F}}=\frac{1}{2 n v_{T} S_{N T}}$. Por outro lado, $\frac{d Q_{D, H D}}{d V_{G F}}$ é obtido com $\frac{d K 1}{d V_{G F}}=\frac{1-n \frac{d V_{D E}}{d V_{G F}}}{2 n v_{T}} e$ $\frac{d K 2}{d V_{G F}}=\frac{1-n \frac{d V_{D E}}{d V_{G F}}}{2 n v_{T} S_{N T}}$ que dependem das derivadas da tensão de dreno efetiva $\left(\mathrm{dV}_{\mathrm{DE}} / \mathrm{dV}_{\mathrm{GF}}\right)$, que é apresentada abaixo e inclui a contribuição da saturação da velocidade dos portadores na tensão de saturação ( $\left.\mathrm{V}_{\mathrm{DSAT}}\right)$. Entretanto, a derivada de $V_{D E}$ depende do termo $A$ que, como mostrado anteriormente (eq. (61)), é função da 
derivada de $\mathrm{Q}_{\mathrm{D}, \mathrm{HD}}$. Visando tornar o modelo explícito, foi utilizada a aproximação $\frac{\mathrm{dV}_{\mathrm{DE}}}{\mathrm{dV}_{\mathrm{GF}}} \cong-\frac{\mathrm{dQ}_{\mathrm{S}, \mathrm{HD}}}{\mathrm{dV}_{\mathrm{GF}}} \cdot \frac{1}{\mathrm{nC}_{\mathrm{oxf}}} 100$ para estimar o termo $\mathrm{A} . \mathrm{Em}$ seguida, o termo $\mathrm{dV}_{\mathrm{DE}} / \mathrm{dV}_{\mathrm{GF}}$ resulta em:

$$
\begin{aligned}
\frac{d V_{D E}}{d V_{G F}}= & \frac{d V_{D S A T}}{d V_{G F}}-\frac{d V_{D S A T}}{d V_{G F}} \frac{1}{\ln \left(1+e^{A T S}\right)} \ln \left[1+e^{A T S\left(1-\frac{V_{D, H D}}{V_{S A T}}\right)}\right] \\
& -V_{\text {SAT }} \times \frac{A_{T S} \cdot e^{A T S\left(1-\frac{V_{D, H D}}{V_{S A T}}\right)} \cdot\left[\frac{d V_{D, H D}}{d V_{G F}} V_{S A T}+\frac{d V_{D S A T}}{d V_{G F}} V_{D, H D}\right]}{V_{D S A T}^{2} \cdot \ln \left(1+e^{A T S}\right) \cdot\left(1+e^{A T S\left(1-\frac{V_{D, H D}}{V_{S A T}}\right)}\right)}
\end{aligned}
$$

onde $V_{S A T}$ é a tensão de saturação em inversão forte ${ }^{106}, \frac{d V_{D S A T}}{d V_{G F}}=\frac{e^{\frac{V_{S A T}-v_{T}}{v_{T}}} \times B}{V_{T} \times+v_{T} e^{\frac{V_{S A T}-v_{T}}{v_{T}}}}$

$$
\begin{aligned}
B= & \frac{v_{\text {sat }} L_{\text {eff }}}{\mu_{0}} \alpha_{S} A+\frac{1}{\sqrt{\left(\frac{v_{\text {sat }} L_{\text {eff }}}{\mu_{n}}\right)^{2}+2 \frac{v_{\text {sat }} L_{\text {eff }} \frac{\left(-Q_{S, H D}\right)}{\mu_{n}}}{C_{\text {oxf }} n}}} \times\left(-\frac{\alpha_{S} A\left(v_{\text {sat }} L_{\text {eff }}\right)^{2}\left(1+\alpha_{S} E_{\text {eff }}\right)^{2}}{\mu_{0}^{2}} \alpha_{S} A\right. \\
& \left.+\frac{\alpha_{S} A v_{\text {sat }} L_{\text {eff }}}{\mu_{0}} \frac{Q_{S, H D}}{C_{\text {oxf }} n}-\frac{v_{\text {sat }} L_{\text {eff }}}{\mu_{n}} \frac{d Q_{S, H D}}{d V_{G F}} \frac{1}{C_{\text {oxf }} n}\right)
\end{aligned} .
$$

Embora a tensão $V_{D, H D}$ possa ser calculada como apresentado em na ref. [99], por simplicidade, sua derivada pode ser aproximada por

$$
\mathrm{V}_{\mathrm{D}, \mathrm{HD}} \cong \frac{\mathrm{V}_{\mathrm{D}}}{1+\frac{\mu_{\mathrm{n}} \mathrm{L}_{\mathrm{LD}}\left(\mathrm{V}_{\mathrm{GT}, \mathrm{HD}}\right)}{\mu_{\mathrm{nLD}} \mathrm{L}_{\text {eff }}\left(\mathrm{V}_{\mathrm{GT}, \mathrm{LD}}\right)}}
$$

onde $\mu_{\mathrm{nLD}}$ é a mobilidade da região fracamente dopada.

Denominando $C=\mu_{n} L_{L D} V_{G T, H D}$ e $D=\mu_{n L D} L_{e f f} V_{G T, L D}$, a diferenciação da eq. (64) com relação a $V_{G F}$ resulta em

$$
\frac{d V_{D, H D}}{d V_{G F}} \cong \frac{-V_{D}\left\{D \cdot\left[L_{L D} V_{G T, H D} \frac{d \mu_{n}}{V_{G F}}+L_{L D} \mu_{n}\right]+C \cdot\left[L_{\text {eff }} V_{G T, L D} \frac{d \mu_{n L D}}{d V_{G F}}+L_{\text {eff }} \mu_{n L D}\right]\right\}}{\left(1+\frac{C}{D}\right)^{2} \times C^{2}}
$$


sendo o termo $\frac{d \mu_{n L D}}{d V_{G F}}$ calculado usando a expressão (61), substituindo $\mu_{0}$ e $\alpha_{s}$ (que são dependentes da concentração de dopantes), pelos valores correspondentes à região fracamente dopada, e considerando $\frac{d Q_{S, L D}}{d V_{G F}} \cong-n C_{o x f}$ e $\frac{d Q_{D, L D}}{d V_{G F}} \cong 0^{100}$.

\subsubsection{Condutância de dreno}

De forma contrária à diferenciação de $I_{D S}$ com relação à $V_{G F}$, variando-se a tensão $V_{D}$, a derivada do termo $\left(\frac{W}{L_{\text {eff }}} \frac{\mu_{n}}{1+V_{D E} \frac{\mu_{n}}{L_{\text {eff }} V_{\text {sat }}}}\right)$ tem grande influência no valor de g. Desta maneira, é importante considerá-lo na diferenciação da eq. (52) com respeito a $V_{D}$.

Assim, a condutância de dreno pode ser expressa pela eq. (66) que, como no caso da equação de $\mathrm{g}_{\mathrm{m}}$, depende das densidades de carga de inversão, sendo $\mathrm{Q}_{\mathrm{D}, \mathrm{HD}}$ calculado com $\mathrm{V}(\mathrm{y})$ igual à tensão de dreno efetiva, $\mathrm{V}_{\mathrm{DE}}$.

$$
\begin{aligned}
g_{D} & =\frac{d l_{D S}}{d V_{D}}=\frac{W}{L_{\text {eff }}} \frac{\mu_{n}}{1+V_{D E} \frac{\mu_{n}}{L_{\text {eff }} V_{\text {sat }}}}\left[V_{T}\left(\frac{d Q_{D, H D}}{d V_{D}}-\frac{d Q_{S, H D}}{d V_{D}}\right)-\frac{Q_{D, H D} \frac{d Q_{D, H D}}{d V_{D}}-Q_{S, H D} \frac{d Q_{S, H D}}{d V_{D}}}{n C_{\text {oxf }}}\right]+\frac{W}{L_{\text {eff }}}\left[\frac{\frac{d \mu_{n}}{d V_{D}}}{1+V_{D E} \frac{\mu_{n}}{L_{\text {eff }} V_{\text {sat }}}}\right. \\
& \left.+\frac{\mu_{n}}{L_{\text {eff }} V_{\text {sat }}}\left(\frac{\frac{d V_{D E}}{V_{D}} \mu_{n}+\frac{d \mu_{n}}{V_{D}} V_{D E}}{\left(1+V_{D E} \frac{\mu_{n}}{L_{\text {eff }} V_{\text {sat }}}\right)^{2}}\right)\right] \cdot\left[v_{T}\left(Q_{D, H D}-Q_{S, H D}\right)-\frac{Q_{D, H D}^{2}-Q_{S, H D}^{2}}{2 n C_{\text {oxf }}}\right]
\end{aligned}
$$

A diferenciação das cargas de inversão com relação à $V_{D}$ é semelhante à diferenciação com relação à $\mathrm{V}_{\mathrm{GF}}$, sendo expressa por 


$$
\frac{d Q_{i}}{d V_{D}}=-4 S_{N T}^{2} \frac{\ln \left[1+\frac{\frac{-Q_{0}}{C_{\text {oxf }}}}{n v_{T} S_{N T}} e^{K 1}+e^{K 2}\right] \cdot\left(\frac{\frac{-Q_{0}}{C_{\text {oxf }}}}{n v_{T} S_{N T}} e^{K 1} \frac{d K 1}{d V_{D}}+e^{K 2} \frac{d K 2}{d V_{D}}\right)}{\sqrt{1+\frac{4}{\left(n C_{o x f} v_{T}\right)}\left[-n C_{o x f} v_{T} S_{N T} \ln \left(1+\frac{\frac{-Q_{0}}{C_{\text {oxf }}}}{n v_{T} S_{N T}} e^{K 1}+e^{K 2}\right]\right]^{2}\left(1+\frac{\frac{-Q_{0}}{C_{\text {oxf }}}}{n v_{T} S_{N T}} e^{K 1}+e^{K 2}\right)}}
$$

Entretanto, neste caso, o efeito de redução da barreira induzida pelo dreno (DIBL) deve ser considerado, através do parâmetro $\sigma$ (seção 2.3.3). Logo, o termo $\frac{d Q_{S, H D}}{d V_{D}}$ é calculado com $\frac{d K 1}{d V_{D}}=\frac{\sigma}{2 n v_{T}}$ e $\frac{d K 2}{d V_{D}}=\frac{\sigma}{2 n v_{T} S_{N T}}$. Para o cálculo de $\frac{d Q_{D, H D}}{d V_{D}}$, $\frac{d K 1}{d V_{D}}=\frac{1}{2 n v_{T}} \cdot\left(\sigma-n \frac{d V_{D E}}{d V_{D}}\right) e \frac{d K 2}{d V_{D}}=\frac{1}{2 n v_{T} S_{N T}} \cdot\left(\sigma-n \frac{d V_{D E}}{d V_{D}}\right)$

Novamente, a derivada da carga de inversão no dreno é dependente da tensão de dreno efetiva, que inclui o efeito de velocidade de saturação, e pode ser obtido pela eq. (62), substituindo as derivadas em relação à $V_{G F}$ pelas correspondentes em relação à $V_{D}$. Novamente, $V_{D, H D}$ foi simplificado através da eq. (64) resultando em

$$
\frac{d V_{D, H D}}{d V_{D}} \cong\left(1+\frac{\mu_{n} L_{L D}\left(V_{G T, H D}\right)}{\mu_{n L D} L_{e f f}\left(V_{G T, L D}\right)}\right)^{-1}
$$

As eqs. (60) e (66) são válidas para qualquer condição de polarização de transistores GC e resultam em expressões bastante precisas para a transcondutância e condutância de dreno de transistores GC SOI, como se demonstrará a seguir.

\subsubsection{Comparação com resultados experimentais}

A fim de verificar a validade das equações desenvolvidas, seus resultados foram comparados a medidas experimentais. Para esta comparação, foram utilizados os mesmos transistores caracterizados anteriormente. Foram utilizados, nas 
comparações, dispositivos com comprimento de canal (L) e 0,5 $\mu \mathrm{m}^{110}$, com diferentes relações $L_{L D} / L$ e largura de canal de $18 \mu \mathrm{m}$. Em todas as comparações realizadas, as curvas experimentais foram obtidas através da diferenciação numérica das curvas de corrente medidas. Utilizando as equações propostas, foram obtidas curvas da transcondutância e condutância de dreno de dispositivos com as mesmas características dos transistores caracterizados. Nas curvas apresentadas, as linhas representam os resultados obtidos através das eqs. (60) e (66) e os símbolos representam os dados experimentais.

A Figura 27 apresenta a comparação entre as curvas de transcondutância em função da sobretensão de condução de dispositivos com três relações $L_{L D} / L: 0,16$; 0,29 e 0,53, obtidas para $V_{D S}=0,1 \vee(A) ; 0,8 \vee(B)$ e $1,5 \vee(C)$. Como pode ser observado nestas curvas, a transcondutância calculada usando a equação proposta apresenta boa concordância com os resultados experimentais, tanto em triodo como em saturação, com erro inferior a $11 \%$, para todos os valores de $\mathrm{V}_{\mathrm{DS}}$ utilizados.
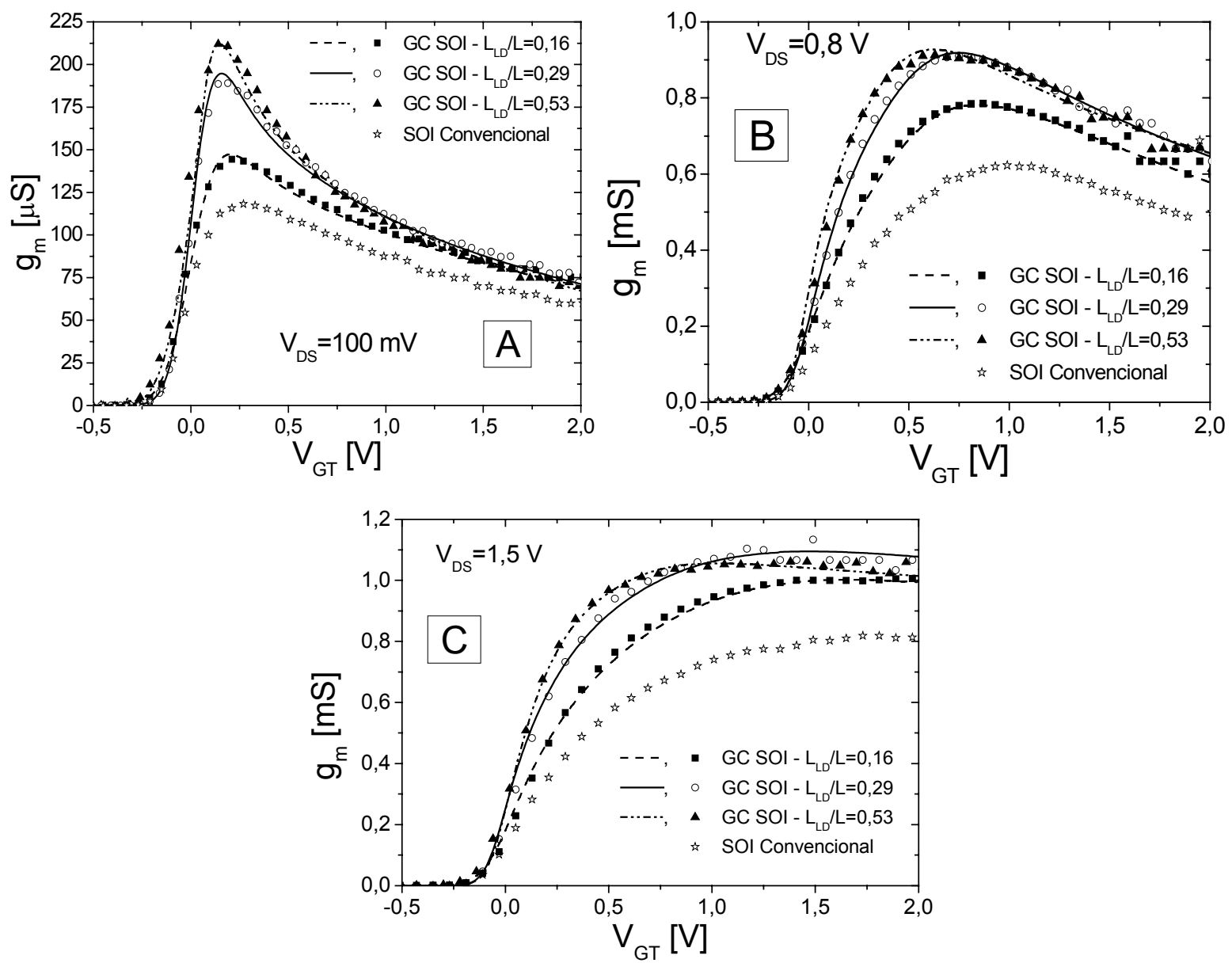

Figura 27 - Transcondutância em função da sobretensão de condução de dispositivos com $\mathrm{L}=0,5 \mu \mathrm{m}$ para diferentes valores de $\mathrm{V}_{\mathrm{DS}}$. 
A partir das curvas apresentadas na Figura 27(A), pode-se notar que, para altas tensões de porta, com o aumento da relação $L_{L D} / L$, há uma maior degradação da transcondutância. Este fenômeno está relacionado à concentração de elétrons na superfície do canal ao longo de seu comprimento para diferentes valores de tensão aplicada à porta. Como mencionado anteriormente, a região fracamente dopada do canal apresenta tensão de limiar negativa e, mesmo com tensão de porta nula, já se encontra em inversão forte. Para valores de tensão de porta inferiores à tensão de limiar, os transistores GC SOI comportam-se de forma semelhante à um transistor SOI convencional com comprimento de canal $L=L_{H D}$. À medida que a tensão $V_{G F}$ é aumentada, a concentração de elétrons nas duas regiões do canal torna-se semelhante e o transistor tende a ter um comportamento similar ao de um transistor SOI convencional de mesmo comprimento total de canal, porém com maior nível de corrente ${ }^{112}$. Além disso, a maior degradação da mobilidade da região fracamente dopada contribui para o aumento da degradação da transcondutância.

Utilizando a eq. (66) e diferenciando numericamente as curvas experimentais da corrente de dreno em função da tensão aplicada ao dreno, foram obtidas as curvas da condutância de dreno, variando a relação $L_{L D} / L$ para um valor fixo de $V_{G T}$ e variando a polarização de porta para um dispositivo com $L_{L D} / L=0,28$, apresentadas na Figura 28. A partir da Figura 28(A) pode-se notar a redução de g proporcionada pela estrutura GC em comparação ao transistor convencional. Pode-se notar, também, que a condutância de dreno é bem descrita pela equação proposta, para diversas condições de polarização, desde a inversão fraca até a inversão forte (Figura 28(B)), exceto na região onde o efeito bipolar parasitário se torna importante e que não é de interesse para a operação de circuitos analógicos. 

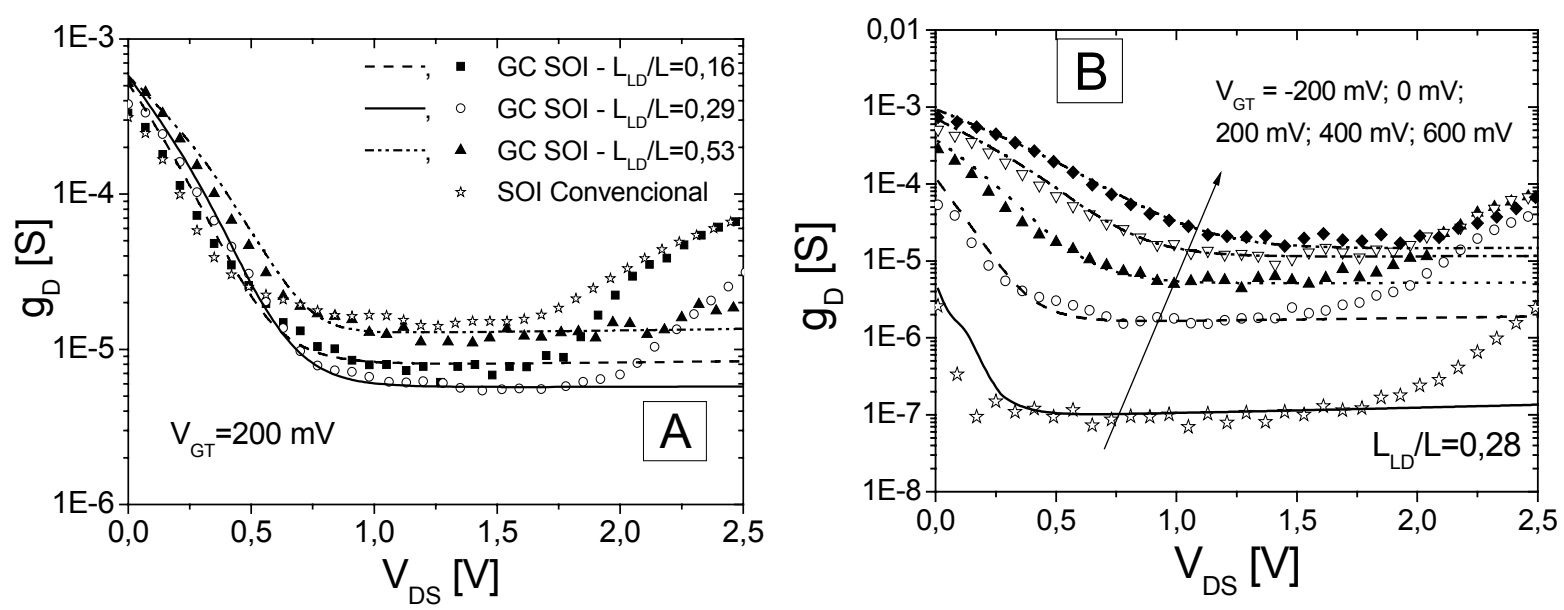

Figura 28 - Comparação entre as curvas experimentais (símbolos) e calculadas (linhas) da condutância de dreno em função da tensão de dreno, para diferentes dispositivos, com $\mathrm{V}_{\mathrm{GT}}=200 \mathrm{mV}(\mathrm{A})$ e para diferentes polarizações, para um transistor GC SOI com $L_{L D} / L=0,28(B)$.

A partir das curvas disponíveis, foram obtidos os valores do ganho de transistores GC SOI, com $V_{\mathrm{DS}}=0,8 \mathrm{~V}$ e 1,5 V, para $\mathrm{V}_{\mathrm{GT}}=200 \mathrm{mV}, 500 \mathrm{mV}$ e $800 \mathrm{mV}$. Utilizando as equações de $g_{m}$ e $g_{D}$, foram obtidas curvas do ganho em função de $\mathrm{V}_{\mathrm{GT}}$, para $\mathrm{V}_{\mathrm{DS}}=0,8 \mathrm{~V}$ e 1,5 V. Os resultados obtidos são apresentados na Figura 29.

Através das curvas apresentadas pode-se notar o aumento do ganho, proporcionado pela presença da região fracamente dopada próxima ao dreno. Considerando o pior caso, há uma melhora de pelo menos $6,5 \mathrm{~dB}$ quando os dispositivos são polarizados com $\mathrm{V}_{\mathrm{GT}}=200 \mathrm{mV}$ e $\mathrm{V}_{\mathrm{DS}}=0,8 \mathrm{~V}$ e de pelo menos 8,5 dB com $\mathrm{V}_{\mathrm{DS}}=1,5 \mathrm{~V}$ e mesmo $\mathrm{V}_{\mathrm{GT}}$.

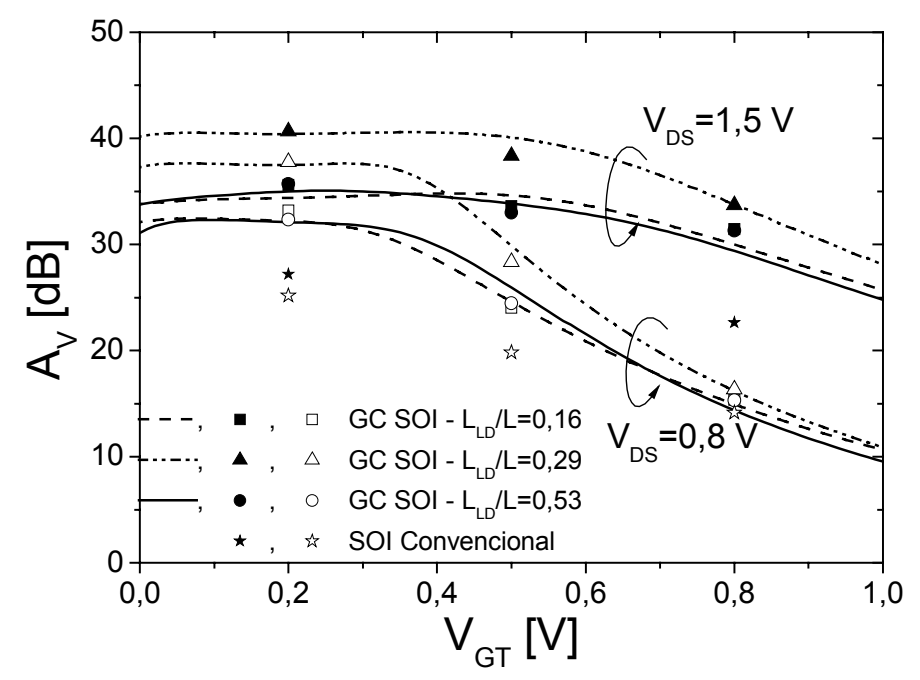

Figura 29 - Ganho de tensão de malha aberta em função de $V_{G T}$ para dispositivos GC SOI e SOI convencional, polarizados com $V_{D S}=0,8$ e 1,5 V. 
A partir dos resultados apresentados, pode-se afirmar que as equações propostas para a obtenção da transcondutância e da condutância de dreno são capazes de descrever, com boa precisão, os resultados obtidos experimentalmente, sob diversas condições de polarização.

SOUZA, M. de; PAVANELLO, M.A.; Charge-Based continuous explicit equations for the Transconductance and Output conductance of Submicron Graded-Channel SOI MOSFET's, Microelectronics Technology and Devices - SBMicro2006, p; 217-226, 2006.

SOUZA, M. de, PAVANELLO, M. A. Charge-Based Continuous Equations for the Transconductance and Output Conductance of Graded-Channel SOI MOSFETs. JICS - Journal of Integrated Circuits and Systems, v.2, p.104 - 110, 2007. 


\section{ESTUDO DE APLICAÇÕES ANALÓGICAS DE TRANSISTORES GC SOI EM BAIXAS TEMPERATURAS}

\subsection{FABRICAÇÃO DE CÉLULAS ANALÓGICAS SIMPLES}

Foram projetados e fabricados dispositivos e blocos analógicos básicos no Laboratório de Microeletrônica da Université catholique de Louvain (UCL), a partir de leiautes desenvolvidos utilizando o programa IC Station ${ }^{113}$. As regras de projeto do processo SOI utilizado no Laboratório de Microeletrônica da UCL foram implementadas em um arquivo de regras compatível com o programa utilizado, permitindo a verificação e correção de eventuais erros nos leiautes projetados.

Transistores SOI totalmente depletados e GC SOI MOSFET foram fabricados na mesma lâmina de modo a promover uma análise comparativa. A posição da implantação iônica (comprimento $\mathrm{L}_{\mathrm{LD}}$ ) em relação ao comprimento de canal do transistor (relação $L_{L D} / L$ ) foi variada, de forma a permitir a obtenção de diferentes dispositivos GC SOI. Os comprimentos de canal dos transistores fabricados foram $2 \mu \mathrm{m}, 1 \mu \mathrm{m}$ e $0,75 \mu \mathrm{m}$, com largura de canal de $20 \mu \mathrm{m}$. Foram também fabricados espelhos de corrente em duas arquiteturas, Cascode e Wilson, além de estruturas com transistores GC SOI e SOI convencional, compostas por 10 transistores idênticos, visando o estudo de descasamento de suas características elétricas.

Na Figura 30 está apresentado o leiaute do chip fabricado. 


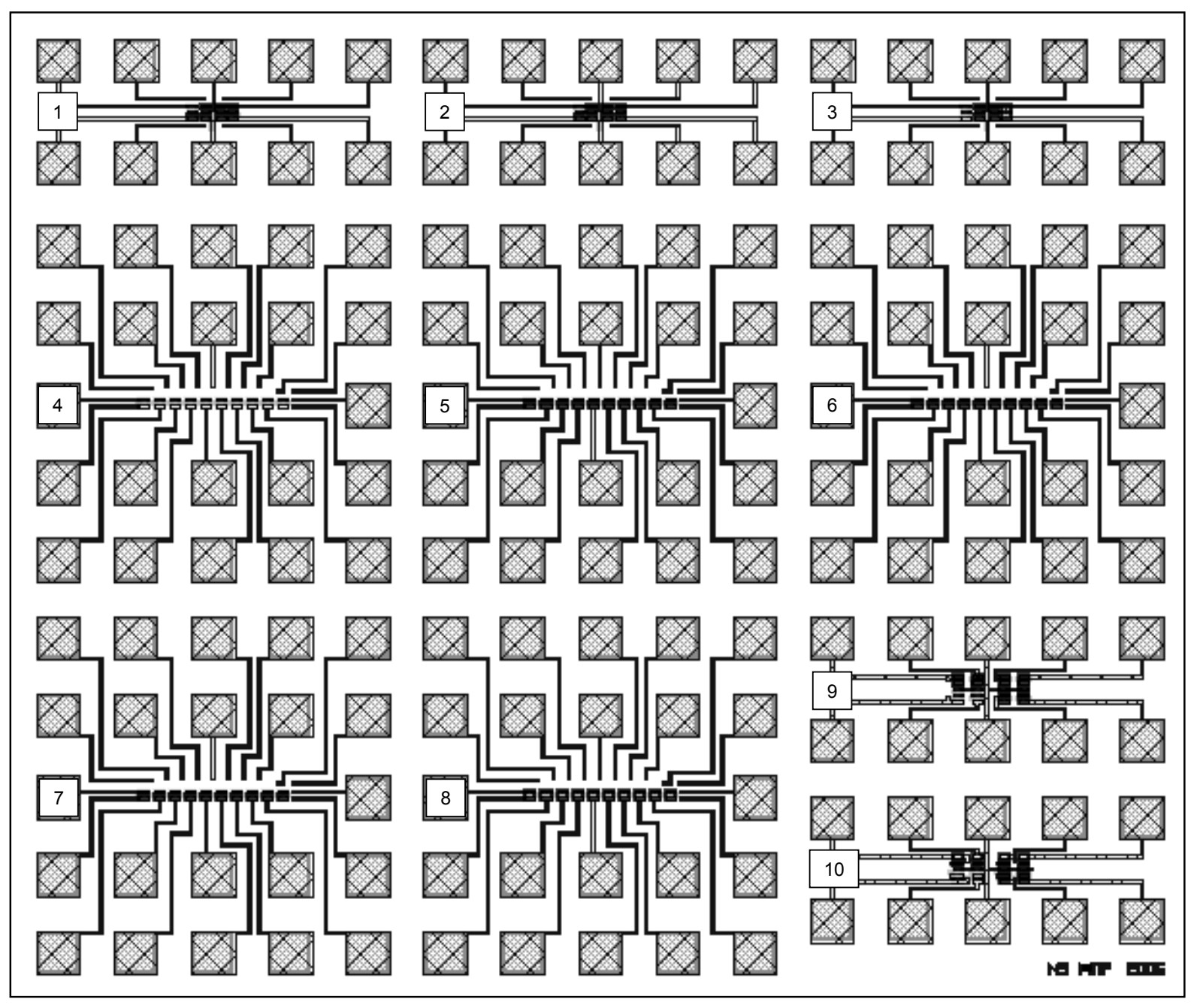

Figura 30 - Leiaute do chip fabricado

As estruturas numeradas na Figura 30 são:

1 - Transistores com L=2 $\mu \mathrm{m}, \mathrm{W}=20 \mu \mathrm{m}$ e $\mathrm{L}_{\mathrm{LD}} / \mathrm{L}=0 ; 0,125 ; 0,25 ; 0,375 ; 0,50 ; 0,625$; 0,75 e 1 ;

2 - Transistores com L=1 $\mu \mathrm{m}, \mathrm{W}=20 \mu \mathrm{m}$ e $\mathrm{L}_{\mathrm{LD}} / \mathrm{L}=0 ; 0,25 ; 0,50 ; 0,75$ e 1 ;

3 - Transistores com L=0,75 $\mu \mathrm{m}, \mathrm{W}=20 \mu \mathrm{m}$ e $\mathrm{LLD} / \mathrm{L}=0 ; 1 / 3 ; 2 / 3$ e 1 ;

4 - Estrutura para o estudo de descasamento com 10 transistores SOI convencionais idênticos e L $=2 \mu \mathrm{m}$ e $\mathrm{W}=20 \mu \mathrm{m}$;

5 - Estrutura para o estudo de descasamento com 10 transistores GC SOI idênticos e $L=2 \mu \mathrm{m}, L_{L D} / L=0,25$ e $W=20 \mu \mathrm{m}$;

6 - Estrutura para o estudo de descasamento com 10 transistores GC SOI idênticos e $L=2 \mu \mathrm{m}, L_{L D} / L=0,375$ e $W=20 \mu \mathrm{m}$; 
7 - Estrutura para o estudo de descasamento com 10 transistores GC SOI idênticos e $L=2 \mu \mathrm{m}, L_{L D} / L=0,50$ e $W=20 \mu m$;

8 - Estrutura para o estudo de descasamento com 10 transistores SOI convencionais idênticos, fracamente dopados, e $L=2 \mu \mathrm{m}$ e $\mathrm{W}=20 \mu \mathrm{m}$;

9 - Espelhos de corrente com configuração Wilson, com GC SOI com L=2 $\mu \mathrm{m}$ e $\mathrm{W}=20 \mu \mathrm{m}, \mathrm{L}_{\mathrm{LD}} / \mathrm{L}=0 ; 0,25 ; 0,375$ e 0,50;

10 - Espelhos de corrente com configuração Cascode, com GC SOI com L=2 $\mu \mathrm{m}$ e $W=20 \mu \mathrm{m}, \mathrm{L}_{\mathrm{LD}} / \mathrm{L}=0 ; 0,25 ; 0,375$ e 0,50;

Nas Figuras 31 e 32 são apresentadas, respectivamente, fotos dos espelhos de corrente e de uma das estruturas para estudo de descasamento fabricados:

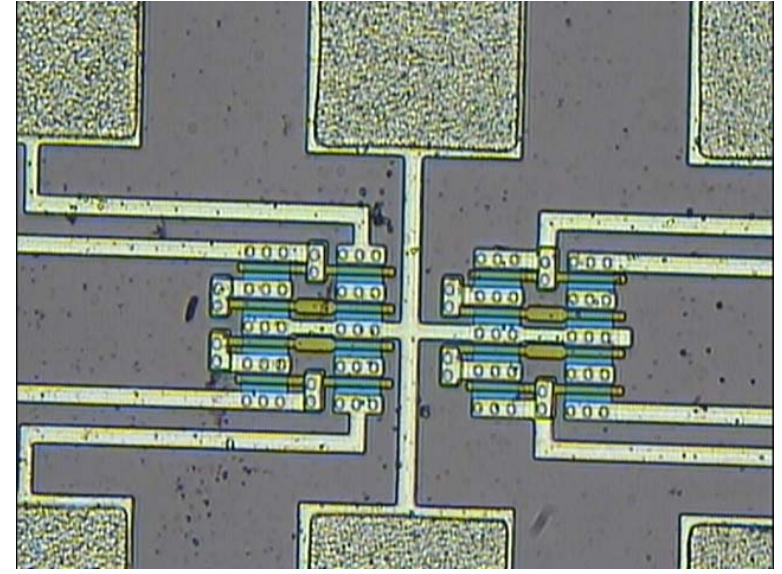

(A)

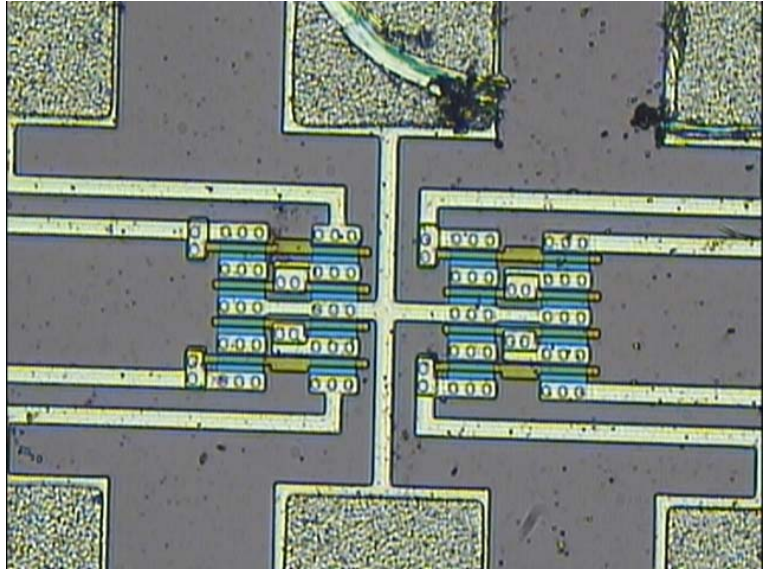

(B)

Figura 31 - Fotos dos espelhos de corrente fabricados, com estruturas Cascode (A) e Wilson (B).
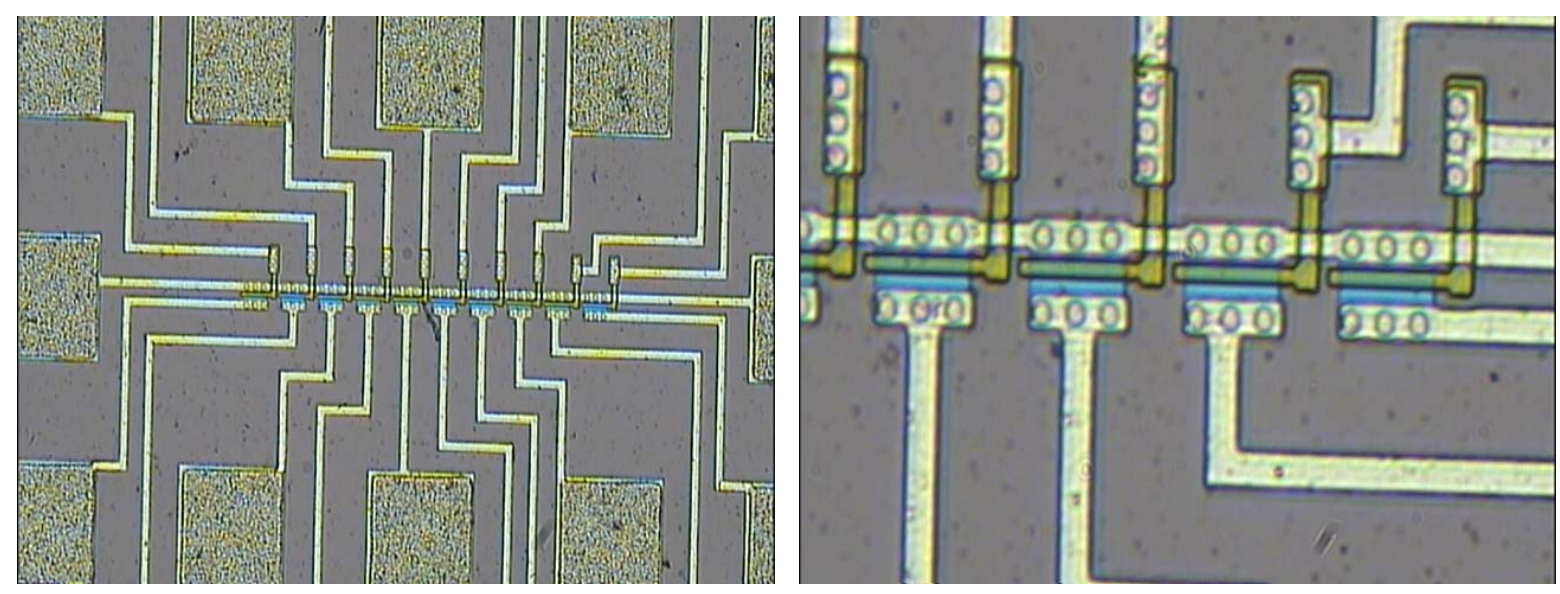

Figura 32 - Fotos de uma das estruturas para estudo de descasamento. 


\subsection{DISTORÇÃO HARMÔNICA}

\subsubsection{Análise da distorção harmônica em temperatura ambiente}

A distorção harmônica é uma variável bastante importante no projeto de células analógicas. Assim, modelos analíticos orientados à simulação de circuitos analógicos utilizando transistores MOS devem descrever adequadamente seu comportamento não-linear, além de assegurar a continuidade das curvas de corrente e de suas derivadas em relação às tensões aplicadas aos seus terminais.

A fim de testar a capacidade do modelo analítico contínuo para transistores GC SOI em descrever suas características de linearidade, utilizamos seus resultados para avaliar a distorção harmônica, através da comparação com resultados obtidos experimentalmente ${ }^{114}$. Foram caracterizados e modelados, em $\mathrm{T}=300 \mathrm{~K}$, transistores GC SOI com $W=18 \mu \mathrm{m}, \mathrm{L}=0,5 \mu \mathrm{m}$ e diferentes relações $\mathrm{L}_{\mathrm{LD}} / \mathrm{L}$. A distorção harmônica total (THD) e as componentes de segunda (HD2) e terceira ordem (HD3) foram adotadas como figuras de mérito nesta comparação. Estes parâmetros foram extraídos utilizando o método da função integral (Integral Function Method - IFM ) ${ }^{82}$, já mencionado na seção 2.4.3.

As curvas $\mathrm{I}_{\mathrm{DS}}$ em função de $\mathrm{V}_{\mathrm{GT}}$, medidas e modeladas, necessárias para a análise de distorção harmônica em saturação, obtidas com $V_{D S}=1,5 V$ são apresentadas na Figura 33. Como esperado, o aumento da relação $L_{L D} / L$ provoca o aumento do nível de corrente, devido à redução do comprimento efetivo de canal. Nesta figura também são apresentadas as curvas da relação $g_{m} / l_{D s}$ que, como mencionado anteriormente, são de grande importância do ponto de vista de circuitos analógicos. Em todas as curvas apresentadas, as linhas representam os dados modelados e os símbolos, os resultados experimentais. 


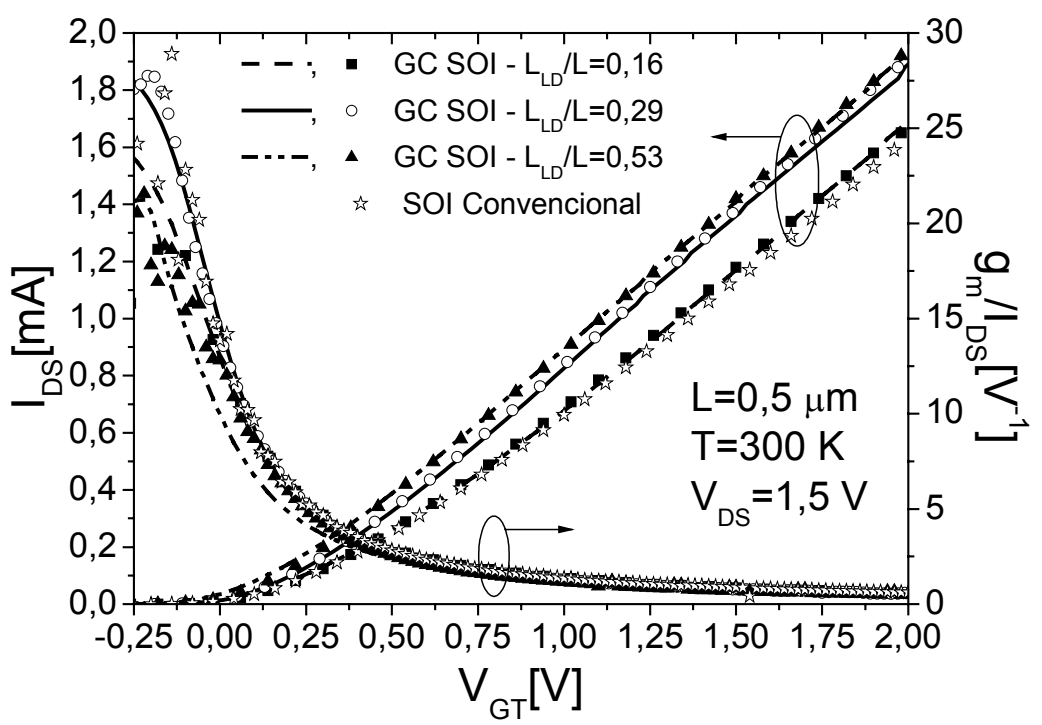

Figura 33 - Curvas $I_{D S}$ em função de $V_{G T}$ obtidas através de medidas (símbolos) e do modelo analítico (linhas), com $V_{D S}=1,5 \mathrm{~V}$.

Conforme discutido anteriormente, o ganho de tensão de malha aberta $\left(A_{V}\right)$ de um transistor MOS é dado pela relação $g_{m} / g_{D}{ }^{115}$. Através das curvas $I_{D S}$ em função de $V_{D S}$, experimentais e modeladas, obtidas com $V_{G T}=200 \mathrm{mV}$, foram extraídos os valores da condutância de dreno dos dispositivos com $V_{D S}=1,5 \mathrm{~V}$, permitindo o cálculo de $A_{V}$. Os resultados são apresentados na Tabela 4, onde podese notar a boa concordância do modelo com os dados experimentais. A partir dos resultados, observa-se que a estrutura GC proporciona aumento do ganho, em comparação com o dispositivo convencional. No pior caso $\left(L_{L D} / L=0,16\right)$, o aumento obtido foi de cerca de $12 \mathrm{~dB}$.

Tabela 4 - Ganho de malha aberta, obtido com $V_{G T}=200 m V$ e $V_{D S}=1,5 \mathrm{~V}$.

\begin{tabular}{ccc}
$\mathrm{L}_{\mathrm{LD}} / \mathrm{L}$ & Experimental $[\mathrm{dB}]$ & Modelo $[\mathrm{dB}]$ \\
\hline Convencional & 24,40 & - \\
\hline 0,16 & 36,22 & 36,03 \\
\hline 0,29 & 39,67 & 39,69 \\
\hline 0,53 & 36,88 & 35,60 \\
\hline
\end{tabular}


Para a análise da distorção, os dispositivos foram considerados como amplificadores de tensão baseados em um único transistor, como ilustrado na Figura 10. A fim de poder comparar valores relativos, devido às diferenças apresentadas na Tabela 4, as figuras de distorção foram normalizadas por $A_{V}$, que foi obtido combinando-se os resultados de $g_{m} / l_{D s}$ com a tensão Early $\left(V_{E A}\right)$ extraída a partir de curvas $I_{D S}$ em função de $V_{D S}$ com diferentes valores de $V_{G T}\left(A_{V}=\frac{g_{m}}{I_{D S}} \cdot V_{E A}\right)$. A Figura 34 apresenta os resultados experimentais do ganho e da tensão Early em função de $\mathrm{g}_{\mathrm{m}} / \mathrm{I}_{\mathrm{DS}}$. A partir destas curvas, pode-se observar que a presença da região fracamente dopada próxima ao dreno, contribui para a elevação de $V_{E A}$, com conseqüente aumento do ganho. Mesmo o transistor $G C$ com $L_{L D} / L=0,53$, que sofre de efeitos de canal curto, apresenta significativa melhora nos valores de $V_{E A}$ e $A_{V}$.

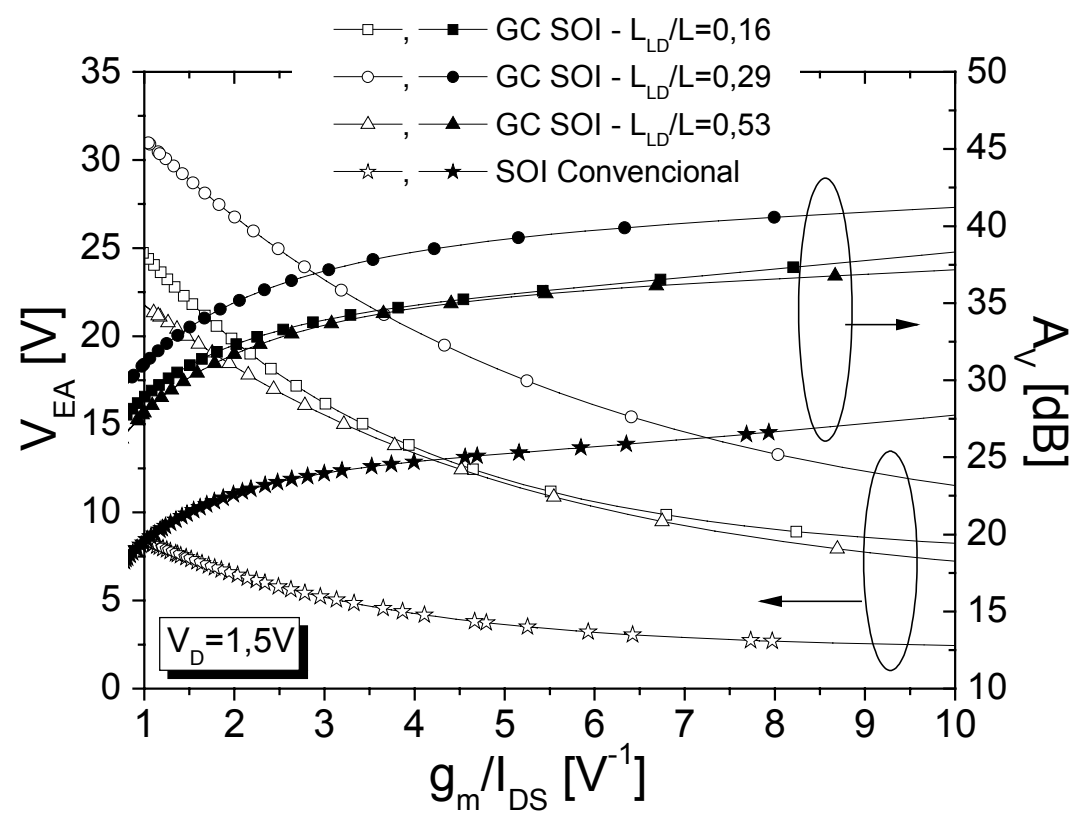

Figura 34 - Tensão Early e ganho de tensão de malha aberta em função da relação $\mathrm{g}_{\mathrm{m}} / \mathrm{I}_{\mathrm{DS}}$ (valores medidos).

Com o intuito de relacionar o consumo de corrente com a linearidade, as curvas de distorção foram traçadas em função de $g_{m} / l_{D S}$. A análise de distorção foi realizada apenas em inversão forte, ou seja, para $g_{m} / I_{D s}$ menor que $10 \mathrm{~V}^{-1}$.

A primeira análise foi realizada fixando a amplitude do sinal de entrada ( $\mathrm{Va}$ ) em $50 \mathrm{mV}$ (100 mV de pico a pico), superposta à tensão de polarização, $V_{\mathrm{GT}}$, que foi variada de 0 a $1,5 \mathrm{~V}$, com $\mathrm{V}_{\mathrm{DS}}=1,5 \mathrm{~V}$. A comparação entre os resultados de THD/AV 
é apresentada na Figura 35A, onde pode-se notar a excelente concordância entre os resultados modelados e medidos, com erro inferior a $5 \mathrm{~dB}$. Para transistores $\mathrm{GC}$ SOI, THD/A $A_{V}$ é praticamente constante com $g_{m} / I_{D s}$; entretanto, para valores de $g_{m} / I_{D S}$ menores que 1 ou $2 \mathrm{~V}^{-1}$, os dispositivos entram na região de triodo, que não é de interesse para a operação de amplificadores.

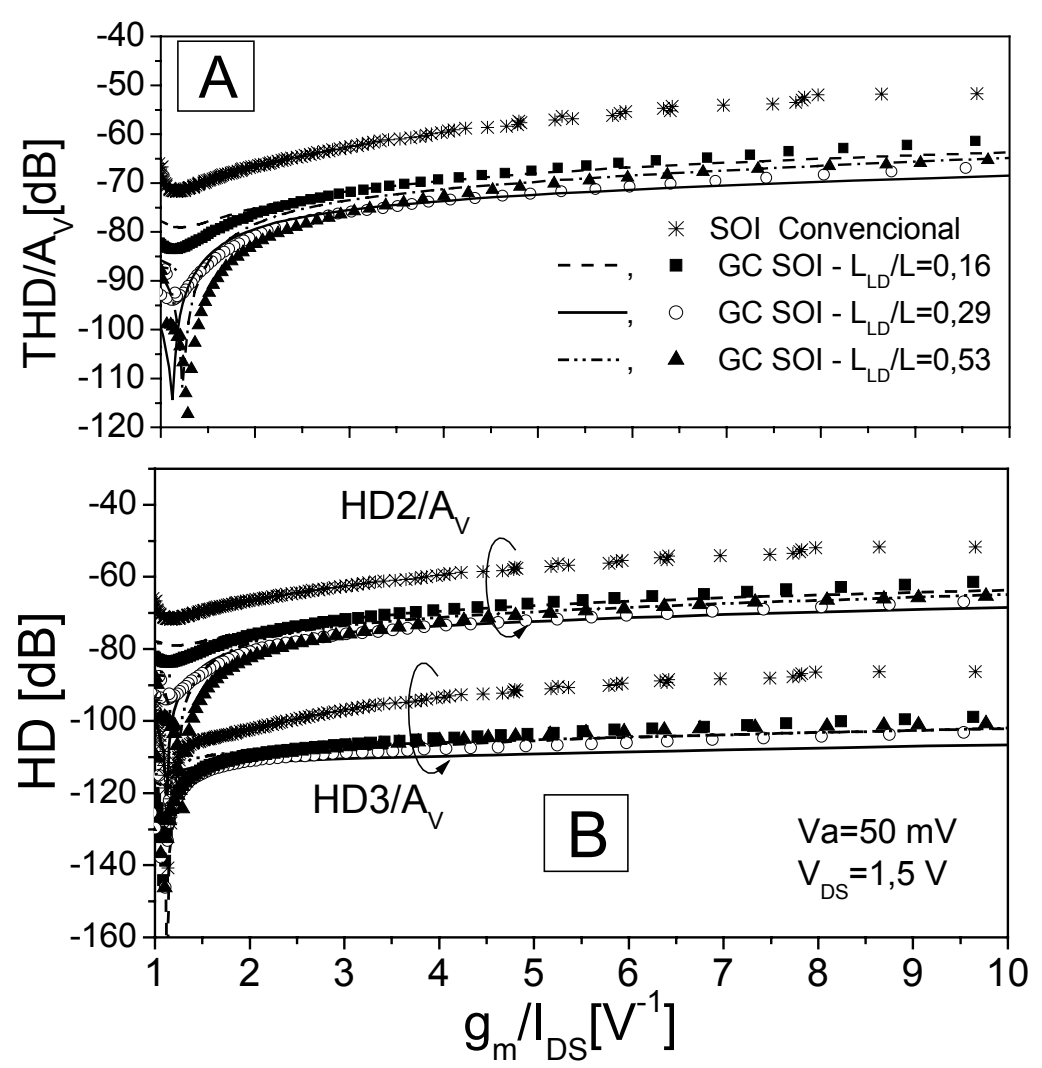

Figura 35 - Comparação entre as curvas THD/Av $(A)$ e HD2/Av e HD3/A $(B)$ obtidas a partir de dados experimentais (símbolos) e modelados (linhas), com Va=50 mV.

A distorção total dos dispositivos operando em saturação é dominada pela distorção de segunda ordem (HD2), independente da arquitetura do canal, como pode ser visto na Figura $35(B)$. Nesta figura, pode-se notar, também, que HD3/Av varia cerca de $10 \mathrm{~dB}$, entre os transistores GC SOI, dependendo da relação $\mathrm{LLD}_{\mathrm{LD}} / \mathrm{L}$. Entretanto, todas as figuras de distorção são sempre melhores nos transistores de canal gradual do que no SOI convencional.

Em todas as figuras de mérito analisadas, os transistores GC SOI apresentam melhora no comportamento, quando comparados ao transistor convencional. THD/A aumenta com o aumento do comprimento efetivo de canal: THD/A $\mathrm{A}_{V}$ do transistor $\mathrm{GC}$ com $L_{L D} / L=0,16$ é cerca de 3 a $5 \mathrm{~dB}$ maior que dos transistores com $L_{L D} / L=0,29$ e 
0,53 para $\mathrm{g}_{\mathrm{m}} / \mathrm{I}_{\mathrm{DS}}=5 \mathrm{~V}^{-1}$, como pode ser visto na Tabela 5 , que apresenta os valores de THD/AV extraídos das curvas apresentadas na Figura 35 , em $g_{m} / l_{D S}=5 V^{-1}$. Comparando os transistores GC ao SOI convencional de mesmo comprimento total de canal, nota-se que todos os dispositivos de canal gradual apresentam redução de THD/Av. Mesmo a presença de um pequena região fracamente dopada $\left(L_{L D} / L=0,16\right)$ proporciona a melhora do ganho e uma redução de $9 \mathrm{~dB}$ em THD/Av, em comparação com o transistor convencional.

Tabela 5 - THD/AV extraído com $g_{m} / I_{D S}=5 V^{-1}$.

\begin{tabular}{ccc}
\hline LLD $/ L$ & Experimental $[d B]$ & Modelo [dB] \\
\hline Convencional & $-57,09$ & - \\
\hline 0,16 & $-66,95$ & $-67,72$ \\
\hline 0,29 & $-71,73$ & $-72,14$ \\
\hline 0,53 & $-70,10$ & $-69,46$ \\
\hline
\end{tabular}

Posteriormente, a distorção dos transistores estudados foi analisada em função da amplitude do sinal de entrada. Neste caso, THD/Av e HD3/Av foram obtidos fixando o valor de $g_{m} / l_{D S}\left(5 V^{-1}\right)$, variando $V a$ de $10 \mathrm{mV}$ a $250 \mathrm{mV}$, como apresentado na Figura 36. 


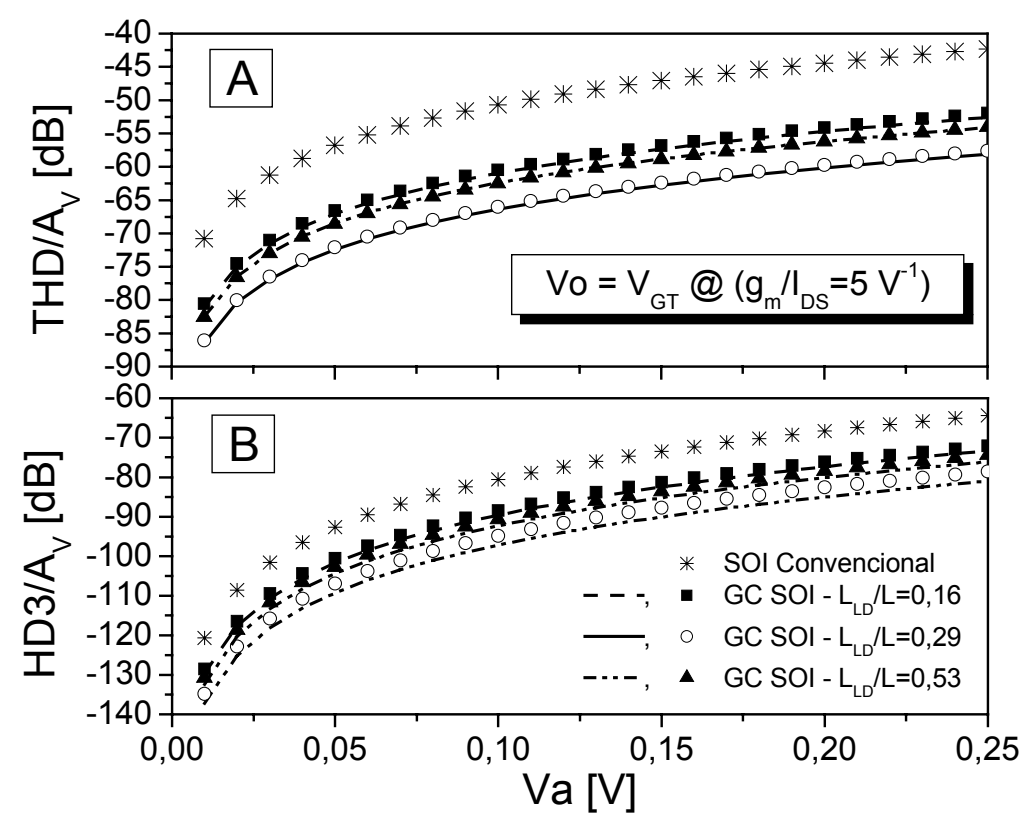

Figura $36-T H D / A_{V}(A)$ e HD3/Av $(B)$ obtidos através de dados experimentais (símbolos) e do modelo analítico (linhas), variando $\mathrm{Va}$ com $\mathrm{gm}_{\mathrm{m}} / \mathrm{l}_{\mathrm{DS}}=5 \mathrm{~V}^{-1}$.

A partir dos resultados apresentados, pode-se notar que, para um determinado nível de distorção fixo, os transistores GC SOI permitem a utilização de amplitudes maiores para o sinal de entrada. Além disso, existe uma boa concordância entre os resultados de distorção obtidos através das curvas modeladas e medidas.

A análise dos resultados obtidos permite afirmar que os transistores GC SOI apresentam maior ganho e menor distorção, quando comparados ao transistor SOI convencional.

\subsubsection{Análise da distorção harmônica em baixas temperaturas}

A distorção harmônica total de transistores operando em baixas temperaturas também foi analisada e os resultados são mostrados na Figura 37, onde são apresentadas as curvas de THD/A $\mathrm{A}_{\mathrm{V}}$, obtidas experimentalmente, para dispositivos GC SOI e SOI convencional operando em $T=300 \mathrm{~K}(\mathrm{~A})$ e $100 \mathrm{~K}$ (B). A partir das curvas apresentadas pode-se notar que a estrutura GC SOI continua apresentando 
vantagens, do ponto de vista da linearidade, quando operando em baixas temperaturas.
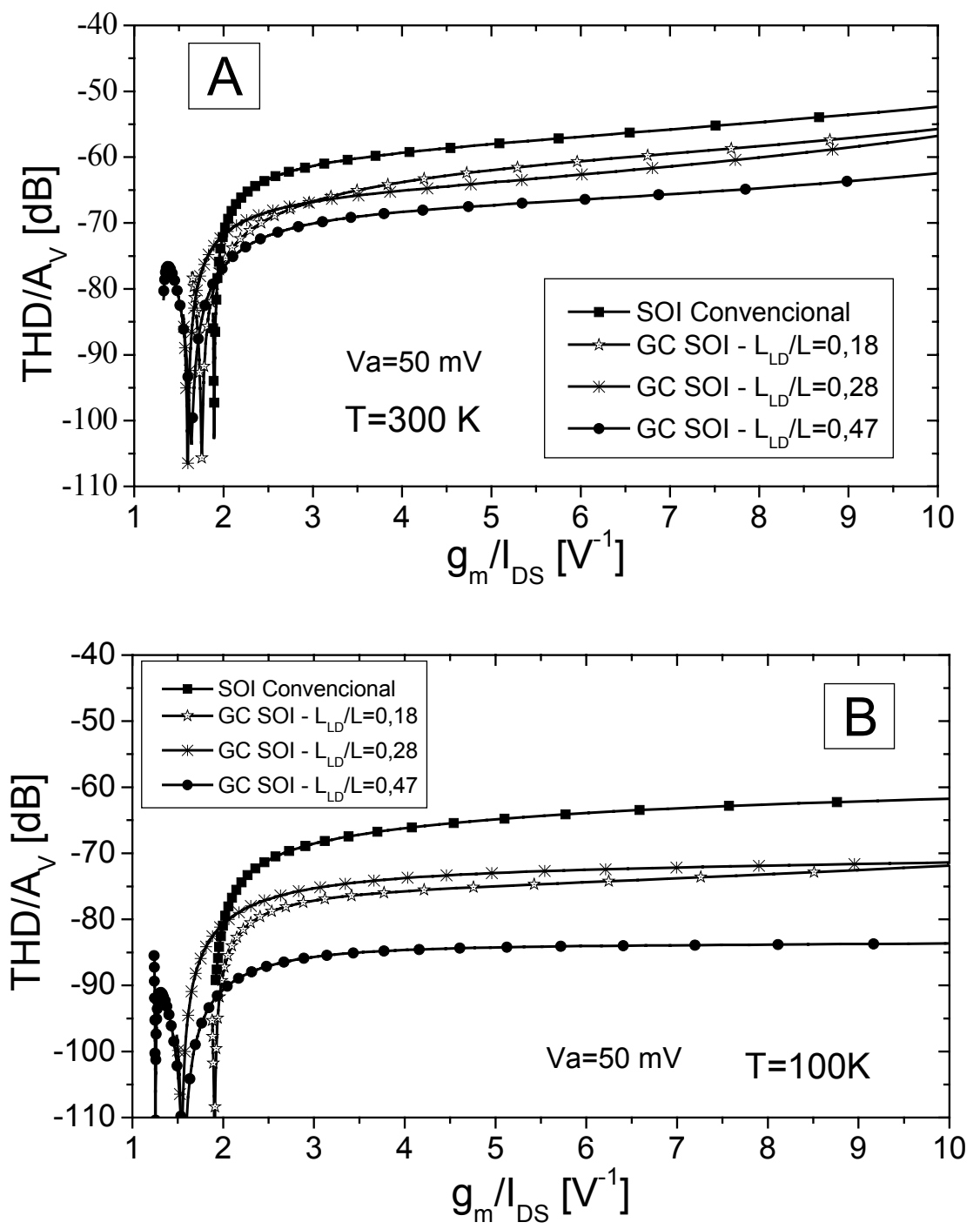

Figura 37 - THD/Av em função de $\mathrm{g}_{\mathrm{m}} / \mathrm{l}_{\mathrm{DS}}$ para dispositivos $\mathrm{GC}$ SOI e SOI convencional operando em $300 \mathrm{~K}(\mathrm{~A})$ e $100 \mathrm{~K}(\mathrm{~B})$, com $\mathrm{V}_{\mathrm{DS}}=1,1 \mathrm{~V}$ e $\mathrm{Va}=50 \mathrm{mV}$.

De acordo com a eq. (51), THD/A $A_{V}$ é função de $g_{D}, g_{m}$ e sua derivada. Utilizando o modelo analítico de transistores GC SOI, as curvas de $\mathrm{I}_{\mathrm{DS}}$ em função de $V_{G F}$ foram ajustadas às experimentais. A partir das curvas modeladas, foram obtidas as curvas de $\mathrm{gm}_{\mathrm{m}}{ }^{2}, \mathrm{dg}_{\mathrm{m}} / \mathrm{dV}_{\mathrm{GF}}$ e $\mathrm{g}_{\mathrm{D}}$, apresentadas nas Figuras 38 e 39 , todas em função de $\mathrm{g}_{\mathrm{m}} / \mathrm{L}_{\mathrm{DS}}$. A análise destas curvas permite afirmar que, para uma dada temperatura, a melhora na linearidade observada nos dispositivos com canal gradual deve-se tanto à redução de $\mathrm{g}_{\mathrm{D}}$, proporcionada pela presença da região fracamente 
dopada próxima ao dreno do transistor, como ao aumento de $\mathrm{g}_{\mathrm{m}}$ (e conseqüentemente, $\mathrm{gm}_{\mathrm{m}}{ }^{2}$ ) causado pela redução do comprimento efetivo de canal. Embora um aumento de $\mathrm{dg}_{\mathrm{m}} / \mathrm{dV}_{\mathrm{GF}}$ tenha sido observado nos dispositivos GC SOI, este não é suficiente para provocar o aumento do nível de distorção em comparação com o dispositivo convencional.

Com a redução da temperatura, apesar da degradação observada em $g_{D}$ em relação à temperatura ambiente, o aumento da transcondutância é responsável pela redução da distorção harmônica total.

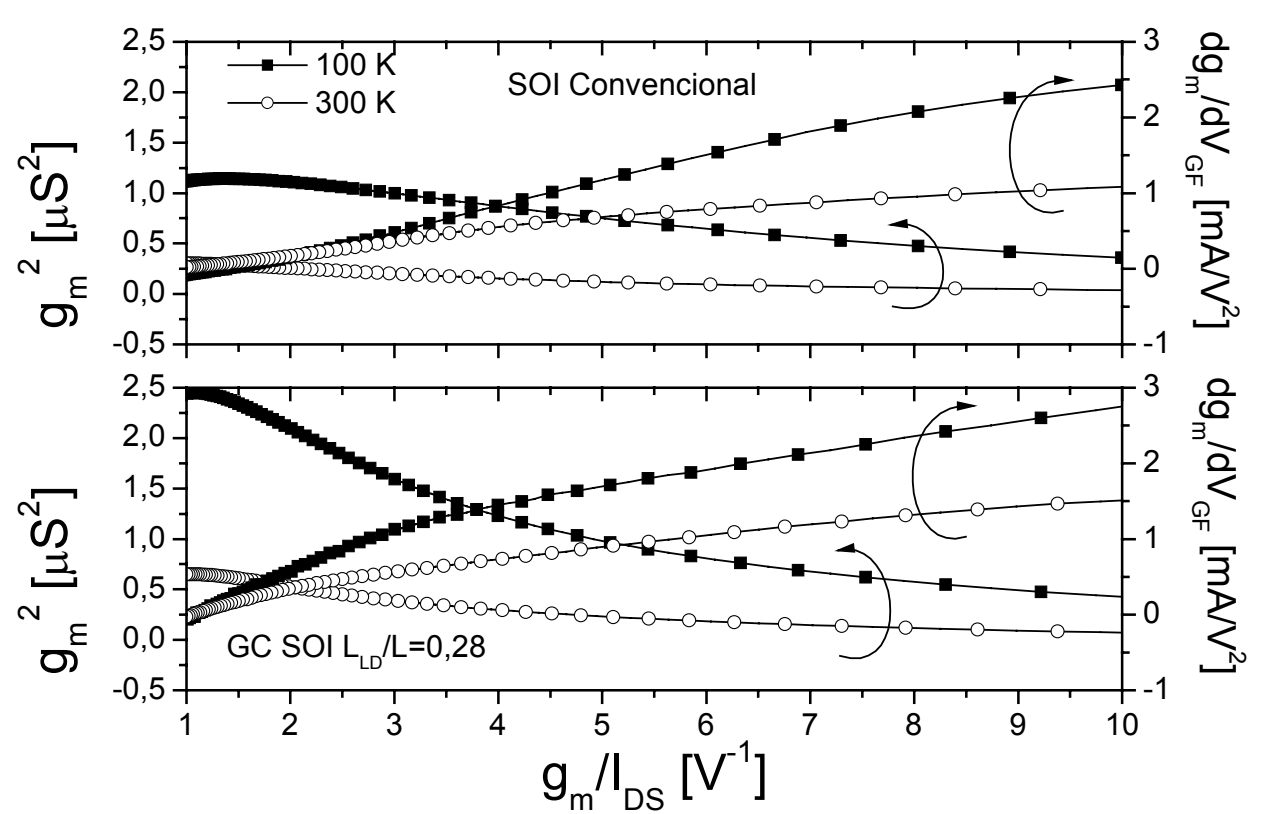

Figura 38 - Curvas $g_{m}{ }^{2}$ e dg $/ d V_{G F}$ em função de $g_{m} / l_{D S}$ obtidas através das curvas modeladas de dispositivos $S O$ I convencional e GC SOI com $L_{L D} / L=0,28 \mathrm{com} V_{D S}=1,1$ $\mathrm{V}$ em $\mathrm{T}=100 \mathrm{~K}$ e $300 \mathrm{~K}$. 


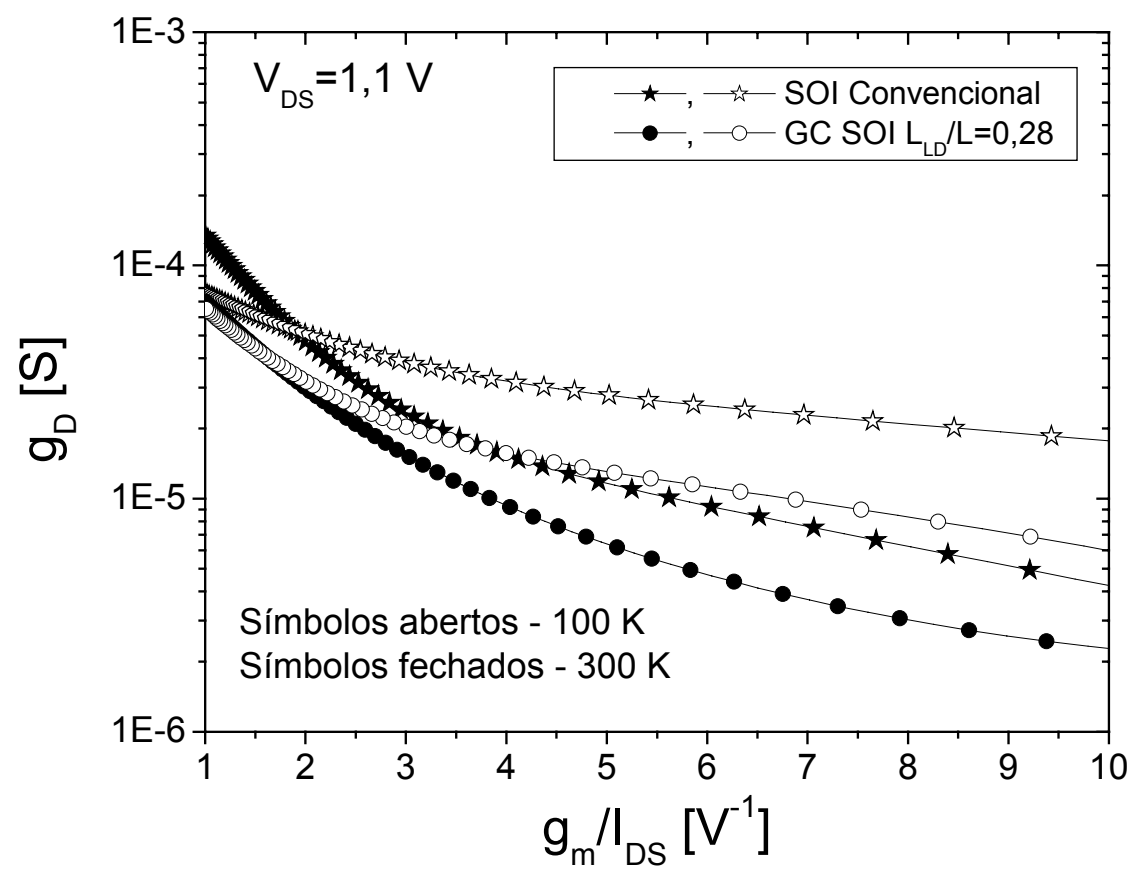

Figura 39 - Curvas da condutância de dreno em função de $\mathrm{g}_{\mathrm{m}} / \mathrm{l}_{\mathrm{DS}}$ obtidas através das curvas modeladas de dispositivos SOI convencional e GC SOI com $L_{L D} / L=0,28$, com $\mathrm{V}_{\mathrm{DS}}=1,1 \mathrm{~V}$ em $\mathrm{T}=100 \mathrm{Ke} 300 \mathrm{~K}$.

SOUZA, M. de; PAVANELLO, M. A., CERDEIRA, A.; FLANDRE, D.; GradedChannel SOI nMOSFET Model Valid for Harmonic Distortion Evaluation. Proc. $25^{\text {th }}$ International Conference on Microelectronis (MIEL 2006), v. 2, p. 509-512, 2006. 


\subsection{ANÁLISE DE DESCASAMENTO EM TRANSISTORES GC SOI}

Descasamento é o processo que causa diferentes desempenhos de dois ou mais dispositivos que possuem projetos idênticos. O casamento de transistores é extremamente importante para circuitos analógicos, uma vez que muitos destes circuitos são baseados no conceito de características semelhantes para dispositivos idênticos ${ }^{116}$.

Dado o potencial do transistor GC SOI para aplicações analógicas demonstrado nas seções anteriores, faz-se necessário o estudo do descasamento de suas características elétricas.

Esta seção apresenta uma análise do descasamento de transistores GC SOI nMOSFETs em comparação com transistores SOI convencionais. Foram estudados o descasamento na corrente de dreno, tanto com transistores operando em triodo como em saturação, e na tensão de limiar. Medidas experimentais são mostradas para suportar a análise, realizada através dos resultados de simulações numéricas bidimensionais e do modelo analítico proposto, procurando identificar as componentes físicas que influenciam o descasamento dos transistores.

\subsubsection{Resultados experimentais ${ }^{117,118}$}

Para esta análise foram utilizadas estruturas de teste com dez transistores com largura de canal de $20 \mu \mathrm{m}$, comprimento de $2 \mu \mathrm{m}$ e diferentes relações $\mathrm{L}_{L \mathrm{D}} / \mathrm{L}$. A distância entre dois transistores consecutivos é igual a $32 \mu \mathrm{m}$ e a distância total entre o primeiro e o último transistor é de $288 \mu \mathrm{m}$. As curvas experimentais foram obtidas utilizando o Sistema de Caracterização de Semicondutores Keithley 4200 e tempo de integração médio.

Em geral, o descasamento relativo na corrente de dreno ( $\left.\Delta \mathrm{I}_{D S} / \mathrm{I}_{\mathrm{DS}}\right)$ é expresso em função do descasamento na tensão de limiar $\left(\Delta \mathrm{V}_{\text {thf }} / \mathrm{V}_{\text {thf }}\right)$ e no fator de corrente $(\Delta \beta / \beta)$, como apresentado na eq. (69), que é válida em inversão forte, com baixa tensão aplicada ao dreno ${ }^{119}$ : 


$$
\frac{\Delta \mathrm{I}_{\mathrm{DS}}}{\mathrm{I}_{\mathrm{DS}}}=\frac{-\Delta \mathrm{V}_{\mathrm{thf}}}{\mathrm{V}_{\mathrm{GF}}-\mathrm{V}_{\mathrm{thf}}}+\frac{\Delta \beta}{\beta}
$$

A variação da tensão de limiar tem origem no descasamento aleatório de parâmetros tais como a concentração de dopantes, espessura da camada de silício e do óxido de porta, entre outros, e é considerada a componente dominante do descasamento de corrente quando dispositivos operam em inversão fraca ${ }^{120}$. Em tecnologias MOS modernas, as espessuras do óxido de porta e da camada de silício apresentam pequena variação, fazendo com que a variação da concentração de dopantes seja a principal fonte do descasamento na tensão de limiar ${ }^{121}$. Esta variação da concentração de dopantes refere-se tanto à concentração original da lâmina como das alterações posteriores, realizadas através da etapa de implantação iônica para ajuste da tensão de limiar.

A tensão de limiar foi extraída a partir do ponto máximo da segunda derivada da curva $I_{D S}$ em função de $V_{G F}$ com baixa polarização de dreno $\left(V_{D S}=25 \mathrm{mV}\right)^{122}$. As curvas da corrente de dreno foram obtidas com passo de $2 \mathrm{mV}$, a fim de aumentar a precisão na extração dos valores de tensão de limiar. $O$ valor médio $\left(V_{\text {thf,mean }}\right)$ e o desvio padrão ( $\left.\sigma \bigvee_{\text {thf }}\right)$ da tensão de limiar obtidos são apresentados na Tabela 6 para transistores SOI convencionais com canal fortemente e fracamente dopados, indicados por $L_{L D} / L=0$ e 1 , respectivamente, e para diferentes transistores GC SOI.

Tabela 6 - Valor médio e desvio padrão da tensão de limiar de transistores SOI convencional e GC SOI.

\begin{tabular}{ccc}
\hline Parâmetro & $\mathrm{V}_{\text {thf,mean }}[\mathrm{mV}]$ & $\sigma_{\mathrm{V}_{\text {thf }}}[\mathrm{mV}]$ \\
\hline $\begin{array}{c}\mathrm{L}_{\mathrm{LD}} / \mathrm{L}=\mathbf{0} \\
\text { (Convencional) }\end{array}$ & 412 & 6 \\
\hline $\mathrm{L}_{\mathrm{LD}} / \mathrm{L}=\mathbf{0 , 1 5}$ & 389 & 6 \\
\hline $\mathrm{L}_{\mathrm{LD}} / \mathrm{L}=\mathbf{0 , 3 5}$ & 380 & 7 \\
\hline $\mathrm{L}_{\mathrm{LD}} / \mathrm{L}=\mathbf{0 , 4 2}$ & 379 & 8 \\
\hline $\mathrm{L}_{\mathrm{LD}} / \mathrm{L}=\mathbf{0 , 7 2}$ & 314 & 9 \\
\hline $\mathrm{L}_{\mathrm{LD}} / \mathrm{L}=\mathbf{1 , 0 0}$ & -313 & 5 \\
\hline
\end{tabular}


Como pode ser visto através dos resultados apresentados, a tensão de limiar média, $V_{\text {thf,mean }}$ diminui em transistores GC SOI com o aumento da relação $L_{L D} / L$, devido à redução do comprimento efetivo de canal. Além disso, os dispositivos com dopagem natural da lâmina $\left(L_{L D} / L=1\right)$ apresentam menor variação de $V_{\text {thf }}$, indicando que a variação da concentração de dopantes aumenta após a implantação iônica para ajuste da tensão de limiar. Além disso, é possível notar que o aumento da relação $L_{L D} / L$, e conseqüente redução do comprimento efetivo de canal, provoca a piora do casamento em $\mathrm{V}_{\mathrm{thf}}$, que é traduzida pelo aumento de $\sigma_{\mathrm{V}_{\mathrm{thf}}}{ }^{123}$.

De acordo com a ref. [124], pode-se entender a redução da tensão de limiar observada em transistores de canal curto como sendo causada pela diminuição da concentração de impurezas no canal. Desta forma, infere-se que a concentração

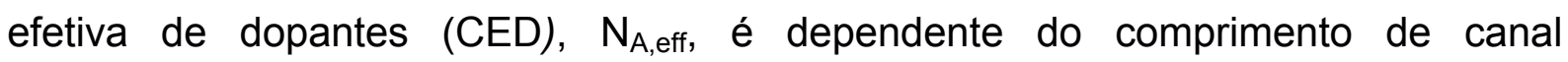
$\left(\mathrm{N}_{\mathrm{A}, \text { eff }}=\mathrm{N}_{\mathrm{A}}-\frac{2 \varepsilon_{\mathrm{Si}}}{\mathrm{q}} \frac{2 \phi_{\mathrm{F}}}{\mathrm{L}^{2}}\right)$ e o descasamento em $\mathrm{V}_{\text {thf }}$ devido à variação do número de dopantes na camada de depleção $\left(\sigma_{V_{\text {thf,CED }}}\right)$ é dado por ${ }^{123}$ :

$$
\sigma_{V_{\text {thf }, C E D}}=\sigma_{V_{\text {thf }}} \frac{1}{\sqrt{1-\frac{4 \phi_{\mathrm{F}} \varepsilon_{\mathrm{Si}}}{\mathrm{qN}_{\mathrm{A}} \mathrm{L}^{2}}}}
$$

A partir desta equação, torna-se clara a influência do comprimento de canal no descasamento provocado na tensão de limiar, conforme observado na Tabela 6.

De acordo com a ref. [121], o desvio padrão da tensão de limiar pode ser expresso por:

$$
\frac{\sigma_{\mathrm{V}_{\text {thf }}}}{\mathrm{V}_{\text {thf,mean }}}=\frac{\sigma_{0 \mathrm{~V}_{\text {thf }}}}{\mathrm{V}_{\text {thf,mean }} \sqrt{\mathrm{W} \times \mathrm{L}}}
$$

onde $\sigma_{0 V_{\text {thf }}}$ é o desvio padrão da tensão de limiar normalizado em relação à área de porta $^{125}$, que está relacionado à concentração de dopantes e às espessuras das camadas de silício e do óxido de porta ${ }^{126}$, como pode ser visto na equação abaixo.

$$
\sigma_{0 V_{\text {thf }}}=\frac{q \cdot t_{o x f} \cdot \sqrt{2 N_{A} t_{S i}}}{\varepsilon_{0} \cdot \varepsilon_{o x}}
$$

A eq. (71) mostra que existe uma proporcionalidade entre o desvio padrão da tensão de limiar e o inverso da raiz quadrada da área de porta do transistor. 
Conforme esperado, quanto maior a área do transistor, menor a variação da tensão de limiar.

A Figura 40 apresenta a relação $\sigma_{V_{\text {thf }}} / V_{\text {thf,mean }}$ em função de $1 / \sqrt{W \times L_{\text {eff }}}$, para o transistor SOI convencional fortemente dopado e diversos transistores GC SOI. Nesta figura os dados experimentais são representados por símbolos, enquanto a linha tracejada apresenta o resultado da regressão linear destes pontos, passando pela origem. Este resultado concorda com dados experimentais apresentados para dispositivos MOS convencionais com diferentes dimensões e espessuras de óxido de porta ${ }^{125,126,127 .}$

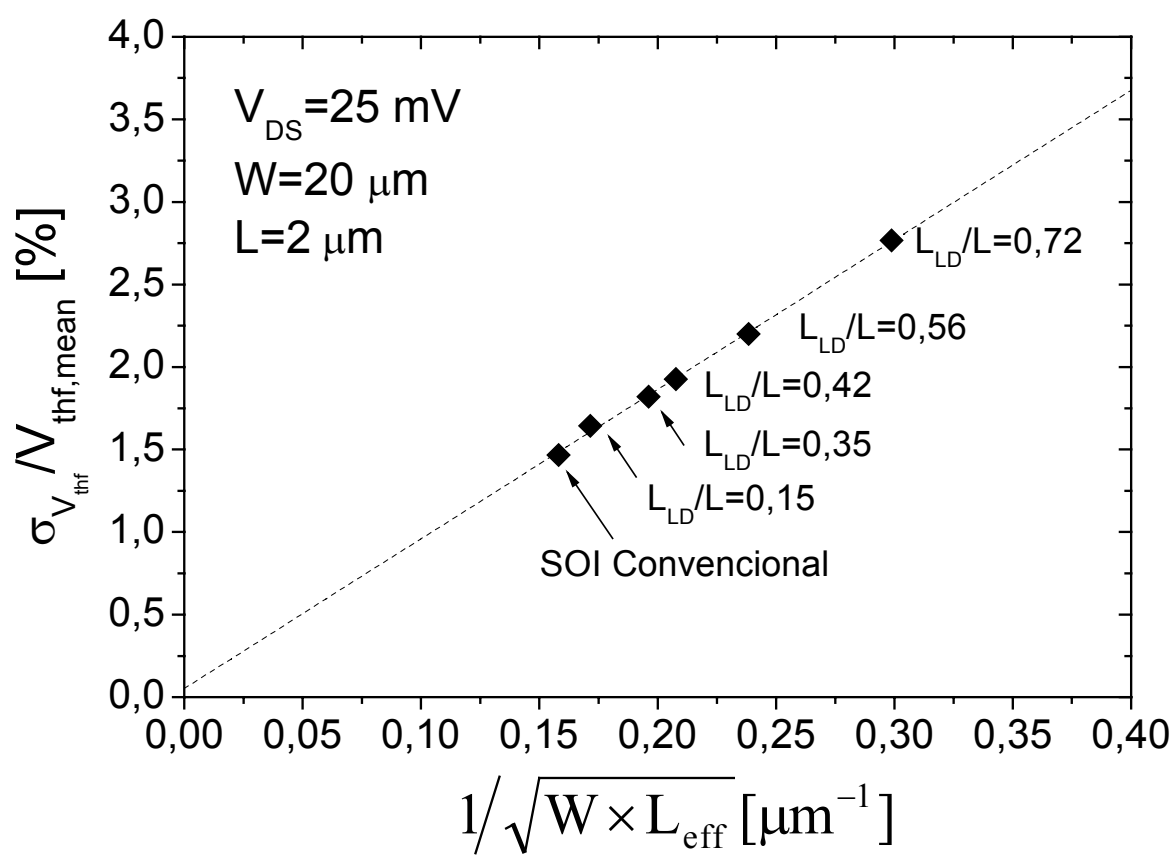

Figura $40-\sigma_{V_{\text {thf }}} / V_{\text {thf,mean }}$ em função da relação $1 / \sqrt{W \times L_{\text {eff }}}$ para transistores de canal gradual com diversas relações $L_{L D} / L$.

A Tabela 7 apresenta os valores de $\sigma_{0 V_{\text {thf }}}$ e $\sigma_{0 V_{\text {thf }}} / V_{\text {thf,mean }}$ calculados a partir dos dados experimentais, utilizando a eq. (71), considerando o comprimento efetivo do canal, $L_{\text {eff. }}$ Como pode ser visto, à medida que a relação $L_{L D} / L$ aumenta (reduzindo $L_{\text {eff }}$ ), o fator $\sigma_{0 v_{\text {tht }}}$ diminui. Esta redução concorda com a consideração proposta na ref. [124], a qual interpreta a ocorrência de efeito de canal curto como 
uma redução da concentração efetiva de dopantes no interior do canal. De acordo com a eq. (72), a diminuição de $N_{A}$ provoca a redução de $\sigma_{0 v_{\text {thf }}}$.

A partir da mesma tabela, pode-se notar que a relação $\sigma_{0 V_{\text {tff }}} N_{\text {thf,mean }}$ permanece praticamente constante à medida que o comprimento da região fracamente dopada do canal é aumentado. Tal resultado indica que, apesar do menor valor médio da tensão de limiar e maior desvio padrão observados nos transistores de canal gradual, qualquer diferença entre o descasamento da corrente de dreno encontrado nos transistores GC com diferentes relações $L_{L D} / L$ e transistores convencionais, não é causada pelo descasamento na tensão de limiar.

Tabela 7 - Valores de $\sigma_{0 V_{\text {thf }}}$ e $\sigma_{0 V_{\text {tht }}} / V_{\text {thf,mean }}$ calculados a partir dos dados experimentais apresentados na Figura 40.

\begin{tabular}{ccc}
\hline Parâmetro & $\sigma_{0 V_{\text {tht }}}[\mathrm{mV} . \mu \mathrm{m}]$ & $\sigma_{0 \mathrm{~V}_{\text {tht }}} / N_{\text {thf,mean }}[\mu \mathrm{m}]$ \\
\hline $\begin{array}{c}\mathrm{L} \mathrm{LD} / \mathrm{L}=\mathbf{0} \\
\text { (Convencional) }\end{array}$ & 39,21 & 0,0927 \\
\hline $\mathrm{L}_{\mathrm{LD}} / \mathrm{L}=\mathbf{0}, \mathbf{1 5}$ & 37,32 & 0,0958 \\
\hline $\mathrm{L}_{\mathrm{LD}} / \mathrm{L}=\mathbf{0 , 4 2}$ & 36,68 & 0,0928 \\
\hline $\mathrm{L}_{\mathrm{LD}} / \mathrm{L}=\mathbf{0 , 5 6}$ & 35,24 & 0,0923 \\
\hline $\mathrm{L}_{\mathrm{LD}} / \mathrm{L}=\mathbf{0}, \mathbf{7 2}$ & 29,12 & 0,0926 \\
\hline
\end{tabular}

A fim de investigar o comportamento da variação do fator de corrente independentemente das diferenças nos valores da tensão de limiar entre os dispositivos com diferentes relações $L_{L D} / L$, as curvas de $\sigma l_{D S} / I_{D S \text {,mean }}$ foram traçadas em função da sobretensão de condução $\left(V_{G T}=V_{G F}-V_{t h f}\right)$, e são apresentadas na Figura 41. 


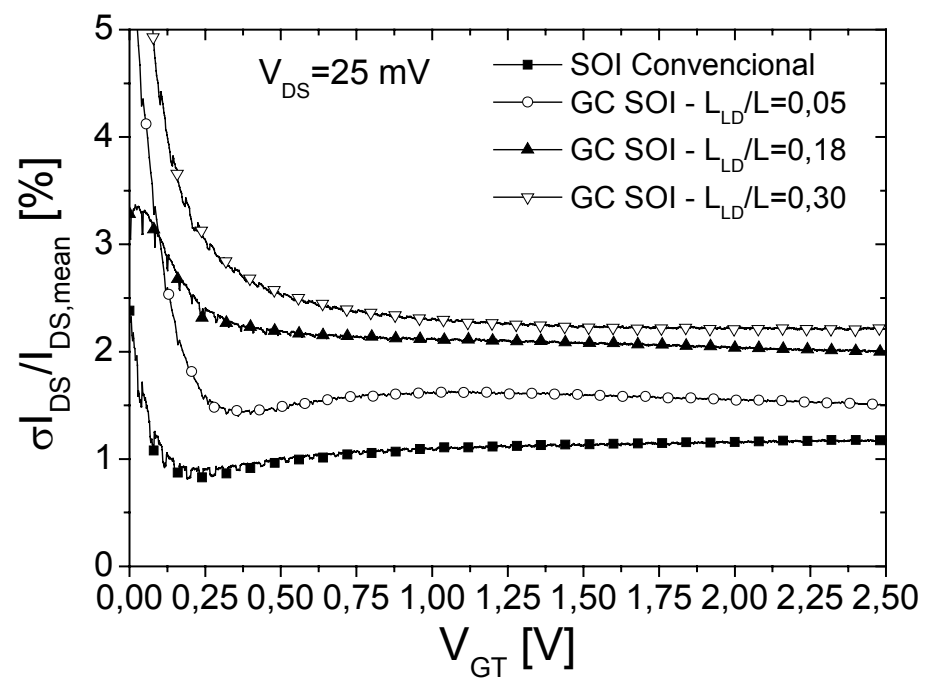

Figura 41 - Desvio padrão da corrente de dreno em função da sobretensão de condução com baixa tensão aplicada ao dreno $\left(\mathrm{V}_{\mathrm{DS}}=25 \mathrm{mV}\right)$ obtido experimentalmente.

A partir das curvas apresentadas, pode-se notar um aumento no desvio padrão à medida que os dispositivos se aproximam da inversão fraca (baixos valores de $\mathrm{V}_{\mathrm{GT}}$ ). Este aumento está relacionado ao descasamento na tensão de limiar, que é dominante neste regime de inversão ${ }^{128}$. À medida que a tensão de porta aumenta, a relação $\sigma \mathrm{I}_{D S} / \mathrm{I}_{\mathrm{DS} \text {, mean }}$ tende a um valor constante, que, no caso do transistor SOI convencional com $L=2 \mu \mathrm{m}$, é de cerca de $1,15 \%$. Além disso, pode-se notar que a variação da corrente de dreno aumenta com $\mathrm{L}_{\mathrm{LD}} / \mathrm{L}$, sugerindo que o fator de corrente em transistores GC SOI é mais susceptível a variações do que em transistores SOI convencionais. As razões para este comportamento, causado pela presença de duas regiões com diferentes concentrações de dopantes, será investigada na próxima seção.

\subsubsection{Análise do descasamento utilizando o modelo analítico contínuo}

Com o objetivo de compreender as componentes que influenciam o descasamento em transistores com canal gradual, resultados oriundos do modelo analítico contínuo de transistores GC SOI ${ }^{99}$ foram utilizados. Para esta análise, a eq. (52) foi reescrita como 


$$
\mathrm{I}_{\mathrm{DS}}=\beta \cdot \mathrm{k}_{\mathrm{Q}}
$$

onde $\beta=\frac{\mu_{n}}{1+V_{D E} \frac{\mu_{n}}{L_{\text {eff }} V_{\text {sat }}}} C_{\text {oxf }} \frac{W}{L_{\text {eff }}}$ e $k_{Q}=\left[v_{T} C_{\text {oxf }}\left(Q_{D, H D}-Q_{S, H D}\right)-\frac{Q_{D, H D}{ }^{2}-Q_{S, H D}{ }^{2}}{2 n}\right]$, onde as densidades de carga de inversão, $Q_{D, H D}$ e $Q_{S, H D}$, são calculadas através da eq. (53).

Desta forma, o desvio padrão relativo na corrente de dreno, desprezando variações na tensão de limiar, pode ser expresso em função do descasamento no fator de corrente $(\Delta \beta / \beta)$ e no termo dependente das cargas de inversão $\left(\Delta \mathrm{k}_{\mathrm{Q}} / \mathrm{k}_{\mathrm{Q}}\right)$ :

$$
\frac{\Delta \mathrm{I}_{\mathrm{DS}}}{\mathrm{I}_{\mathrm{DS}}}=\frac{\Delta \beta}{\beta}+\frac{-\Delta \mathrm{k}_{\mathrm{Q}}}{\mathrm{k}_{\mathrm{Q}}}
$$

Os parâmetros do modelo analítico foram extraídos como apresentado na ref. [99], a fim de ajustar curvas experimentais típicas de transistores convencionais e GC SOI com $W / L=20 \mu \mathrm{m} / 2 \mu \mathrm{m}$ e $L_{L D} / L=0,25,0,40$ e 0,53. Após o ajuste das curvas da corrente de dreno dos transistores com suas dimensões de projeto, seu comprimento efetivo de canal foi variado e o desvio padrão e os termos da eq. (73) foram analisados. Foi imposta uma variação de comprimento de canal de $\pm 30 \mathrm{~nm}$, que é próxima à variação observada experimentalmente $(1,15 \%$, ou $23 \mathrm{~nm})$, caso a variação do fator de corrente $(\beta)$ seja considerada como única responsável pela variação da corrente de dreno. Foi verificado que a variação da mobilidade não é o parâmetro dominante no desvio padrão de $\beta$ e, por este motivo, esta foi mantida constante nesta análise, assim como a tensão de limiar e a inclinação de sublimiar. A Figura 42 apresenta os resultados obtidos para dispositivos operando em região linear, mostrando as curvas $\frac{\sigma \mathrm{I}_{\mathrm{DS}}}{\mathrm{I}_{\mathrm{DS}_{\text {mean }}}}, \frac{\sigma \beta}{\beta_{\text {mean }}}$ e $\frac{\sigma \mathrm{k}_{\mathrm{Q}}}{\mathrm{k}_{\mathrm{Q}_{\text {mean }}}}$ em função de $\mathrm{V}_{\mathrm{GF}}$ com $\mathrm{V}_{\mathrm{DS}}=100$ $\mathrm{mV}$. 


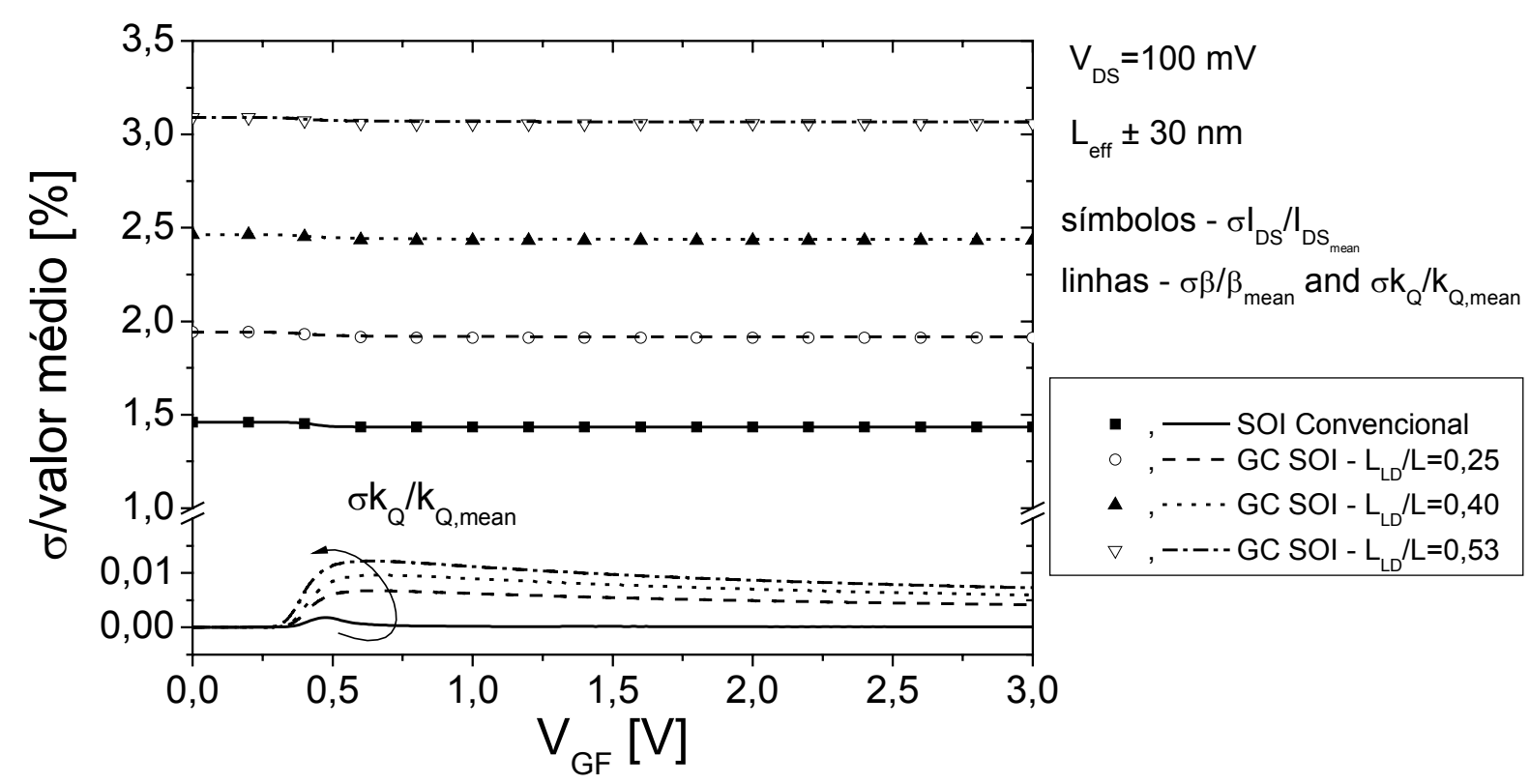

Figura 42 - Desvio padrão da corrente de dreno obtida através do modelo analítico e de seus termos $\beta$ e $\mathrm{k}_{\mathrm{Q}}$ em função da tensão aplicada à porta com $\mathrm{V}_{\mathrm{DS}}=100 \mathrm{mV}$.

Uma breve análise destas curvas indica que o descasamento na corrente de dreno, em região linear, é causada pela variação de $\beta$ e não pela variação de $\mathrm{k}_{\mathrm{Q}}$. Vale ressaltar que a relação $\sigma \mathrm{I}_{\mathrm{DS}} / \mathrm{I}_{\mathrm{DS} \text {,mean }}$ coincide com a variação percentual imposta em $L_{\text {eff. }}$ Uma vez que o comprimento efetivo de canal diminui com o aumento da relação $\mathrm{LLD}_{\mathrm{LD}} \mathrm{L}$, torna-se claro que, para uma variação fixa do comprimento efetivo, o descasamento será maior nos dispositivos GC SOI do que em dispositivos convencionais com o mesmo comprimento de máscara.

A Figura 43 apresenta $\sigma I_{D S} / I_{D S \text {,mean }}$ em função da corrente de dreno normalizada, obtida com $V_{D S}=1,5 \mathrm{~V}$, com as mesmas considerações feitas anteriormente (mobilidade, tensão de limiar e inclinação de sublimiar constantes e $\left.\mathrm{L}_{\text {eff }} \pm 30 \mathrm{~nm}\right)$. 


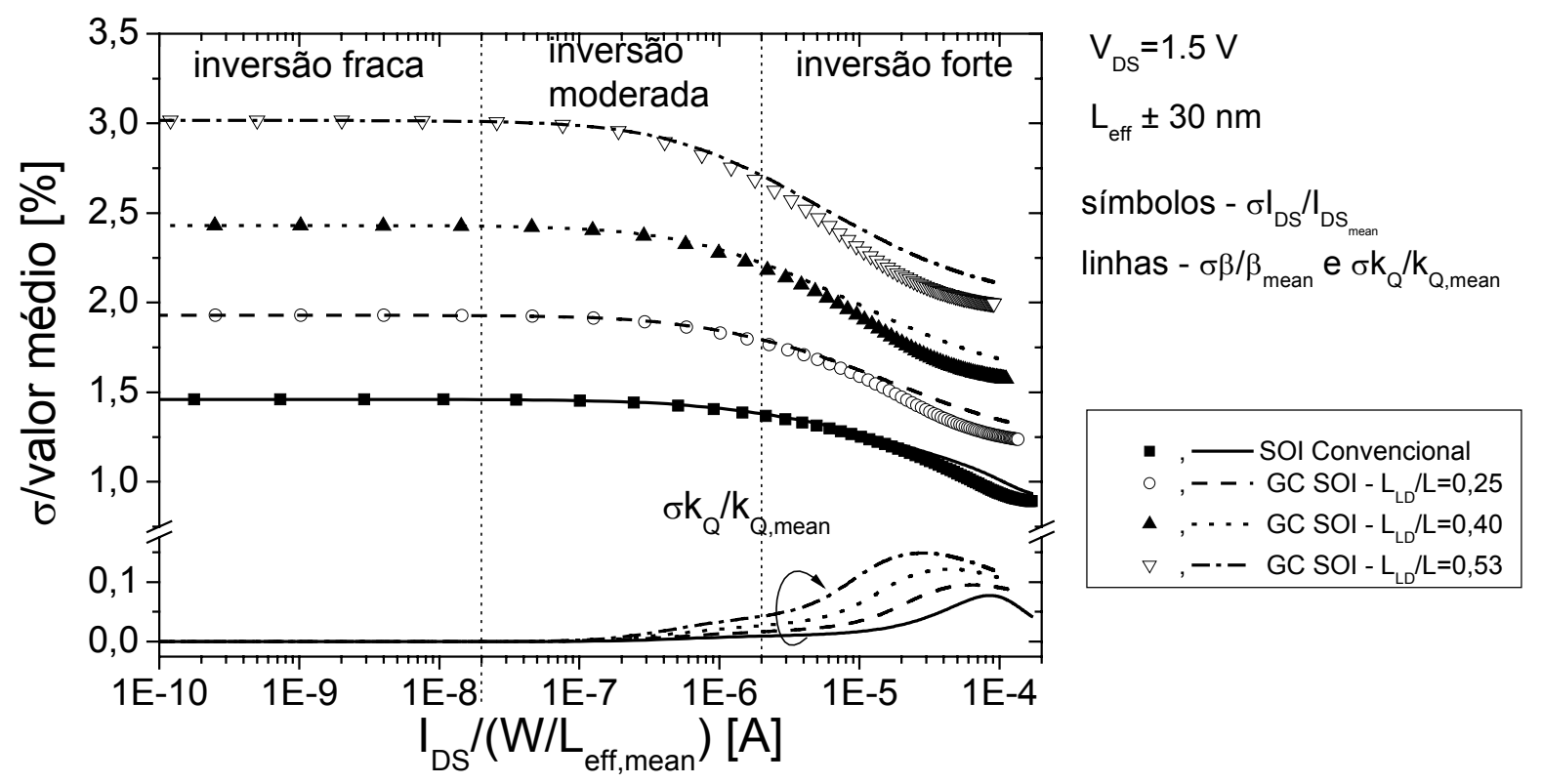

Figura 43 - Desvio padrão da corrente de dreno obtida através do modelo analítico e de seus termos $\beta$ e $k_{Q}$ em função da corrente de dreno normalizada, em saturação $\left(V_{D S}=1,5 \mathrm{~V}\right)$.

A partir desta figura, pode-se notar que o descasamento na corrente de dreno, em saturação, permanece constante (e próxima ao descasamento em região linear) em inversão fraca e moderada. Em inversão forte, o descasamento tende a diminuir, devido à redução de $\sigma \beta / \beta$,mean, causada pelo aumento do desvio da tensão de saturação (e conseqüentemente de $\mathrm{V}_{\mathrm{DE}}$ ), que compensa parte da variação do comprimento efetivo de canal. Além disso, o aumento de $\sigma \bigvee_{D S, S A T} / V_{D S, S A T, m e a n}$, apresentado na Figura 44, influencia o termo $k_{Q}$ na eq. (73) (através de $Q_{D, H D}$ ),

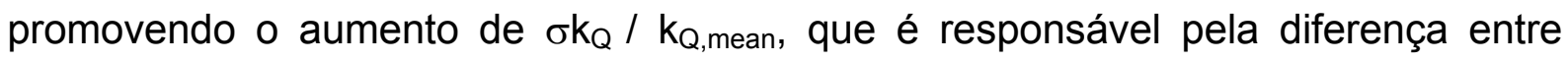
$\sigma I_{D S} / I_{D S \text {, mean }}$ e $\sigma \beta / \beta$, mean observada quando o dispositivo opera em inversão forte. 


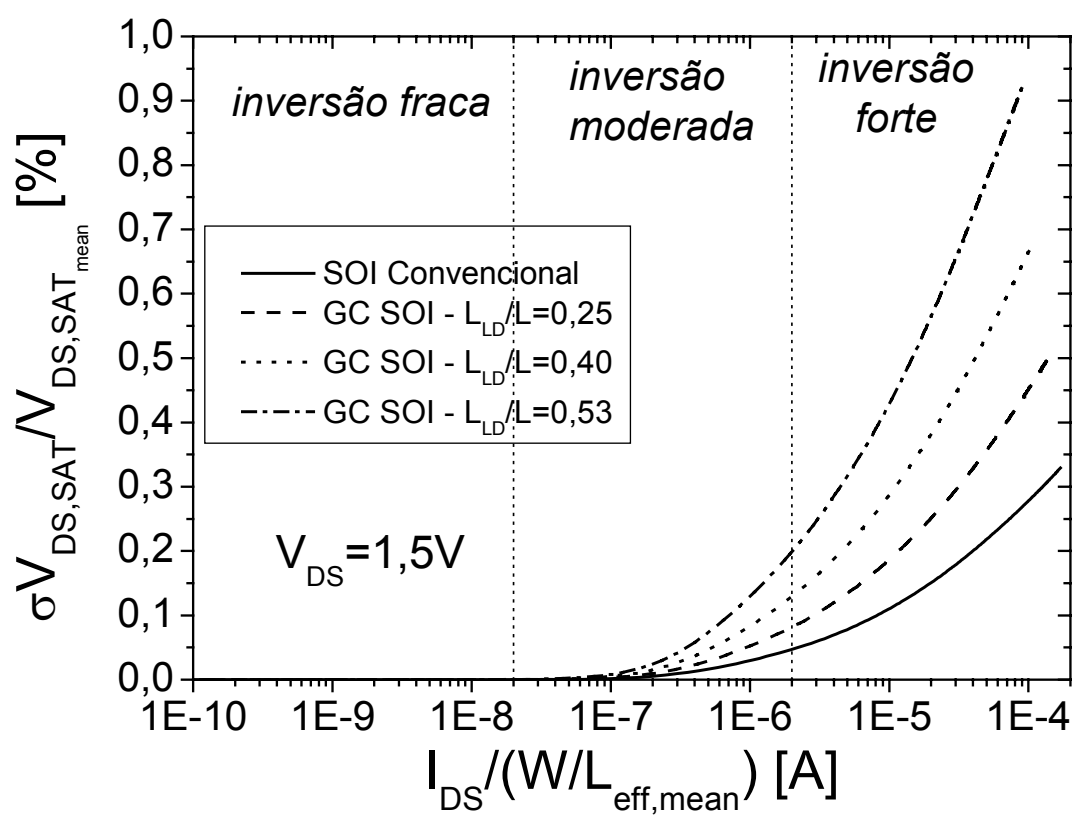

Figura 44 - Desvio padrão da tensão de saturação obtida através do modelo analítico em função da corrente de dreno normalizada, em saturação $\left(V_{D S}=1,5 \mathrm{~V}\right)$.

A variação da tensão de saturação tende a ser maior em transistores com canal gradual do que em transistores convencionais, como mostrado na Figura 44, devido à sua dependência com o comprimento efetivo de canal, em inversão forte ${ }^{100}$.

\subsubsection{Resultados das simulações numéricas bidimensionais}

Simulações numéricas bidimensionais utilizando o simulador Atlas foram ajustadas aos dados experimentais. Os dispositivos foram simulados considerando transição abrupta entre as regiões fortemente e fracamente dopadas e as mesmas características dos dispositivos medidos. Uma vez que a análise realizada a partir do modelo analítico indicou que, em região linear, o descasamento é dominado pela variação do comprimento de canal, pode-se concluir, a partir dos resultados experimentais, que o desvio médio no comprimento de canal, em dispositivos SOI convencionais, é de aproximadamente 1,15\%. Desta forma, o dispositivo SOI convencional foi simulado com suas dimensões de projeto e considerando uma variação de comprimento de canal de $\pm 23 \mathrm{~nm}$. A comparação entre os dados experimentais (símbolos) e simulados (linhas) são apresentados na Figura 45. 


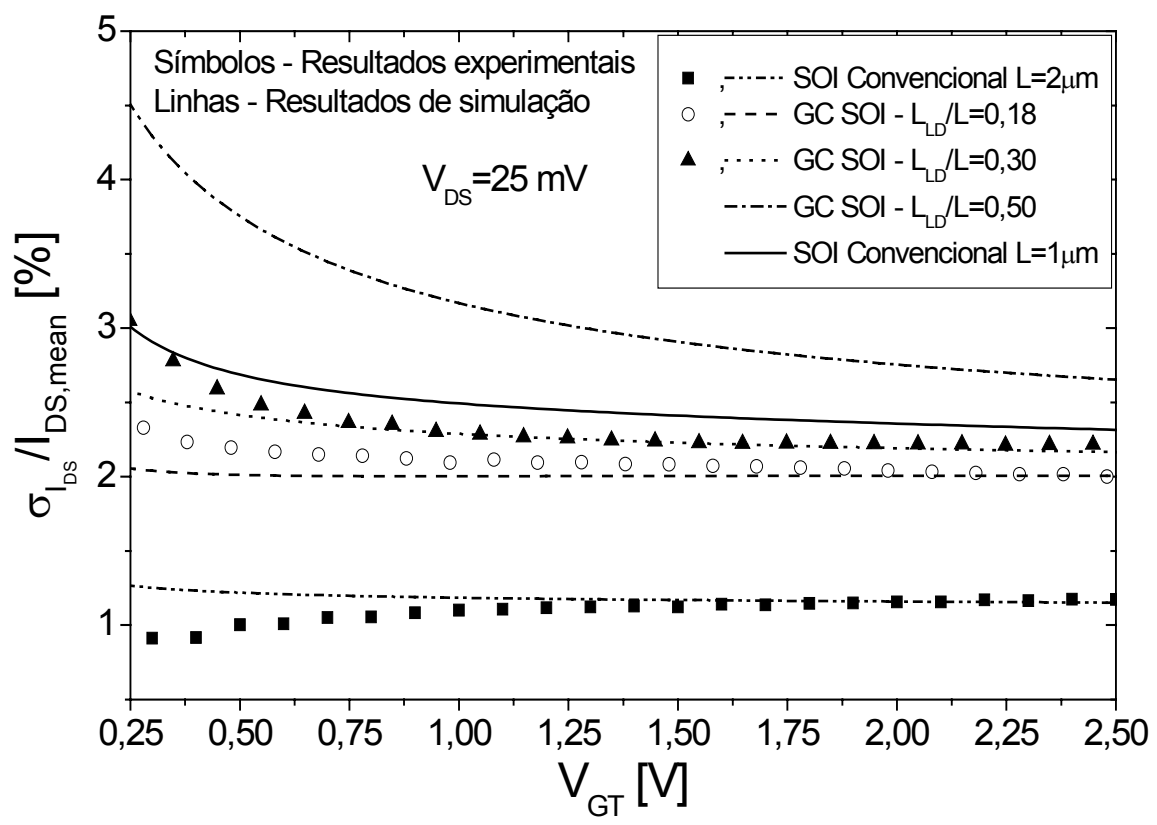

Figura 45 - Comparação entre o desvio padrão da corrente de dreno medida e simulada em função da sobretensão de condução em região linear $\left(\mathrm{V}_{\mathrm{DS}}=25 \mathrm{mV}\right)$.

Em seguida, a mesma variação do comprimento de canal foi imposta ao comprimento de máscara do transistor $\mathrm{GC} S \mathrm{SO}$ com $\mathrm{LLD}_{\mathrm{LD}} / \mathrm{L}=0,30$. Entretanto, $\mathrm{O}$ resultado obtido para $\sigma \mathrm{I}_{D S} / \mathrm{I}_{\mathrm{DS} \text {,mean }}$ foi próximo ao valor do desvio padrão do transistor SOI convencional, indicando que existe uma componente adicional na variação de $\beta$. Esta componente adicional está relacionada ao descasamento do comprimento efetivo de canal, causado pela difusão lateral de dopantes da região fortemente dopada para a região com concentração natural da lâmina e/ou ao desalinhamento na definição de LLD durante o processo. Assim, além da variação do comprimento total do canal, a posição da transição entre as duas regiões com comprimento de canal diferentes foi variada em $\pm 10 \mathrm{~nm}$, resultando na curva apresentada na Figura 45. A mesma variação foi aplicada ao transistor GC SOI com $\mathrm{L}_{\mathrm{LD}} / \mathrm{L}=0,18$, apresentando boa concordância com os resultados experimentais. Um transistor SOI convencional com $L=1 \mu \mathrm{m}$ e um GC SOI com $L=2 \mu \mathrm{m}$ e $L_{L D} / L=0,50$ também foram simulados e os resultados são apresentados no mesmo gráfico.

Vale ressaltar que, apesar da semelhança entre os comprimentos efetivos dos dispositivos SOI convencional de $1 \mu \mathrm{m}$ e o $\mathrm{GC}$ com $L=2 \mu \mathrm{m}$ e $L_{L D} / L=0,50$, este último apresenta maior descasamento, devido à variação adicional de $\beta$, causada pela 
variação da posição da transição entre as regiões fortemente e fracamente dopadas no canal do transistor GC.

Posteriormente, a relação $\sigma \mathrm{I}_{\mathrm{DS}} / \mathrm{I}_{\mathrm{DS} \text {,mean }}$ foi obtida em saturação, com $V_{D S}=1,5 \mathrm{~V}$. As curvas resultante são apresentadas na Figura 46, na qual são mostradas as curvas $\frac{\sigma \mathrm{I}_{\mathrm{DS}}}{\mathrm{I}_{\mathrm{DS}_{\text {mean }}}}$ em função de $\mathrm{I}_{\mathrm{DS}} /\left(\mathrm{W} / \mathrm{L}_{\text {eff }}\right)$. A partir dos resultados apresentados nesta figura, pode-se notar que o casamento melhora à medida que o dispositivo se aproxima da inversão forte, concordando com os resultados obtidos a partir da análise realizada com o modelo analítico. Entretanto, ao contrário do que foi observado com o modelo, em inversão fraca e moderada, $\sigma \mathrm{I}_{\mathrm{DS}} / \mathrm{I}_{\mathrm{DS} \text {,mean }}$ não tende à variação percentual imposta ao comprimento de canal. Esta diferença é causada

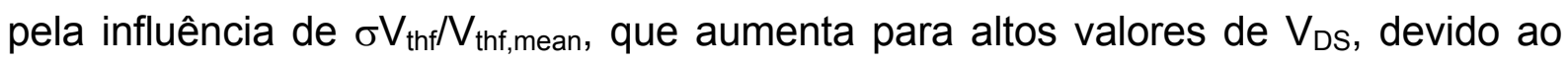
efeito de DIBL, que foi desprezado na análise baseada no modelo analítico.

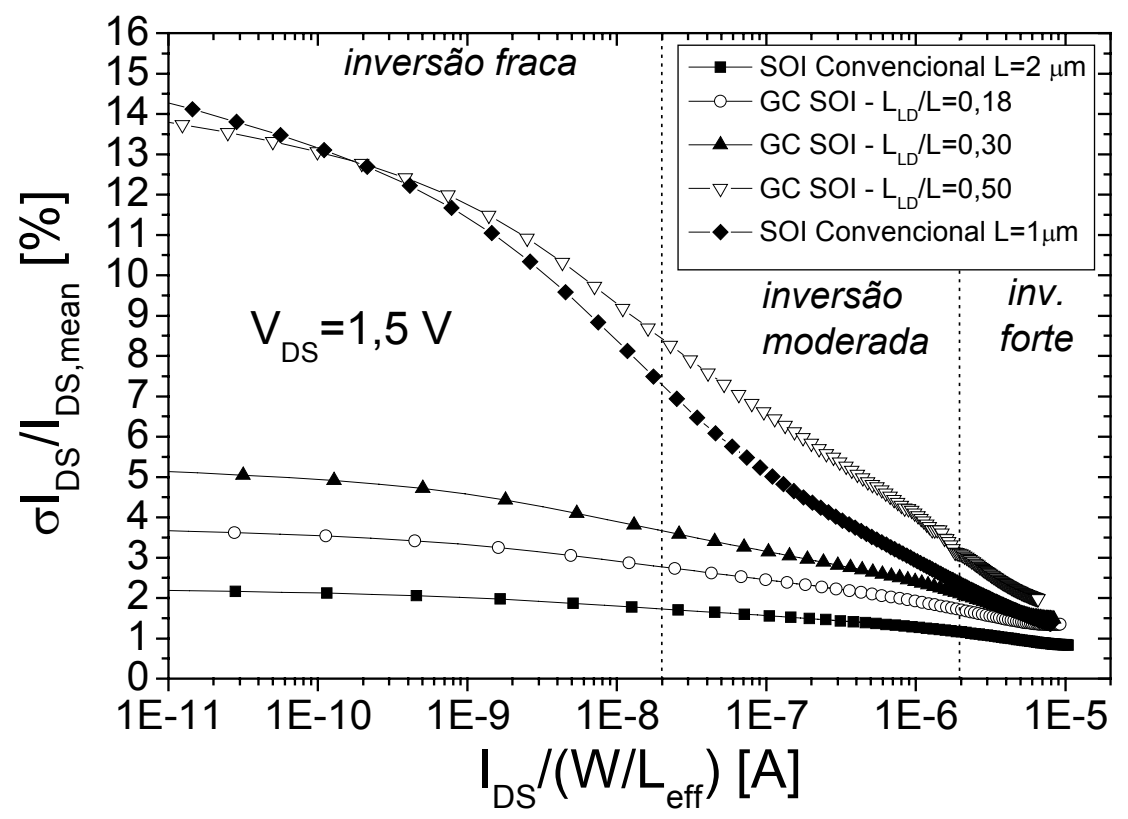

Figura 46 - Desvio padrão da corrente de dreno simulada em saturação $\left(V_{D S}=1,5 \mathrm{~V}\right)$ em função da corrente de dreno normalizada. 


\subsubsection{Análise experimental do descasamento em função da temperatura}

Após a análise do descasamento nas características de transistores de canal gradual operando em temperatura ambiente, a temperatura destas estruturas foi variada, entre $380 \mathrm{~K}$ e $200 \mathrm{~K}$. Para este estudo foram caracterizados transistores SOI convencionais e GC com comprimento total de canal de $2 \mu \mathrm{m}$. Os transistores com canal gradual apresentam relações $L_{L D} / L=0,24 ; 0,38$ e 0,48 , que correspondem a comprimentos efetivos de canal de 1,52 $\mu \mathrm{m}, 1,24 \mu \mathrm{m}$ e 1,04 $\mu \mathrm{m}$, respectivamente.

Na Figura 47 são apresentados os valores médios da tensão de limiar dos transistores caracterizados em função de $L_{\text {eff, }}$ para diferentes temperaturas, extraídos a partir do pico da segunda derivada das curvas $I_{D S}$ em função de $V_{G F}$, medidos com $V_{D S}=25 \mathrm{mV}$. Os resultados apresentados nesta figura permitem observar o aumento da tensão de limiar com a redução da temperatura, bem como o fato de que os valores médios obtidos para o transistor convencional e os diversos transistores de canal gradual permanecem praticamente inalterados, indicando que a influência dos efeitos de canal curto sobre $\bigvee_{\text {thf }}$ nestes transistores é desprezível.

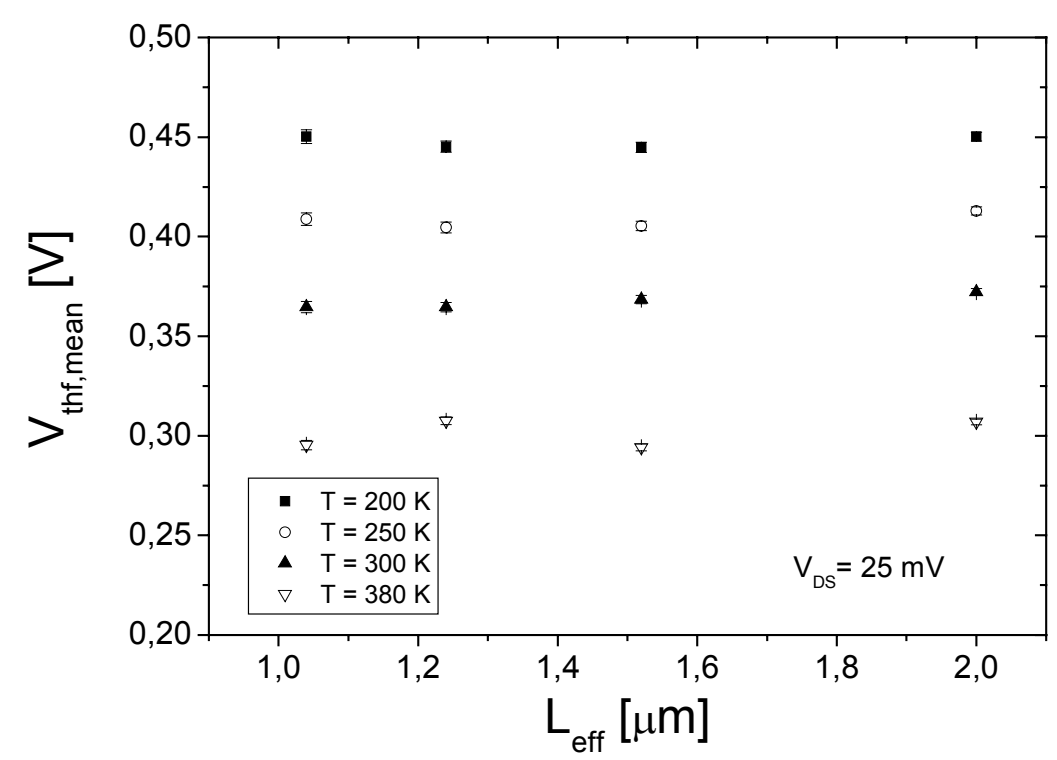

Figura 47 - Valor médio da tensão de limiar em função do comprimento efetivo de canal para diferentes temperaturas. 
A Figura 48 apresenta o desvio padrão da tensão de limiar em função do comprimento efetivo de canal para diferentes temperaturas. A partir dos resultados apresentados, pode-se notar que o aumento de $\sigma_{\mathrm{V}_{\text {thf }}}$ com a redução do comprimento efetivo de canal, observado inicialmente em temperatura ambiente, ocorre também para temperaturas mais baixas. Além disso, a redução da temperatura causa a piora do casamento de $V_{\text {thf }}$ em todas as estruturas analisadas.

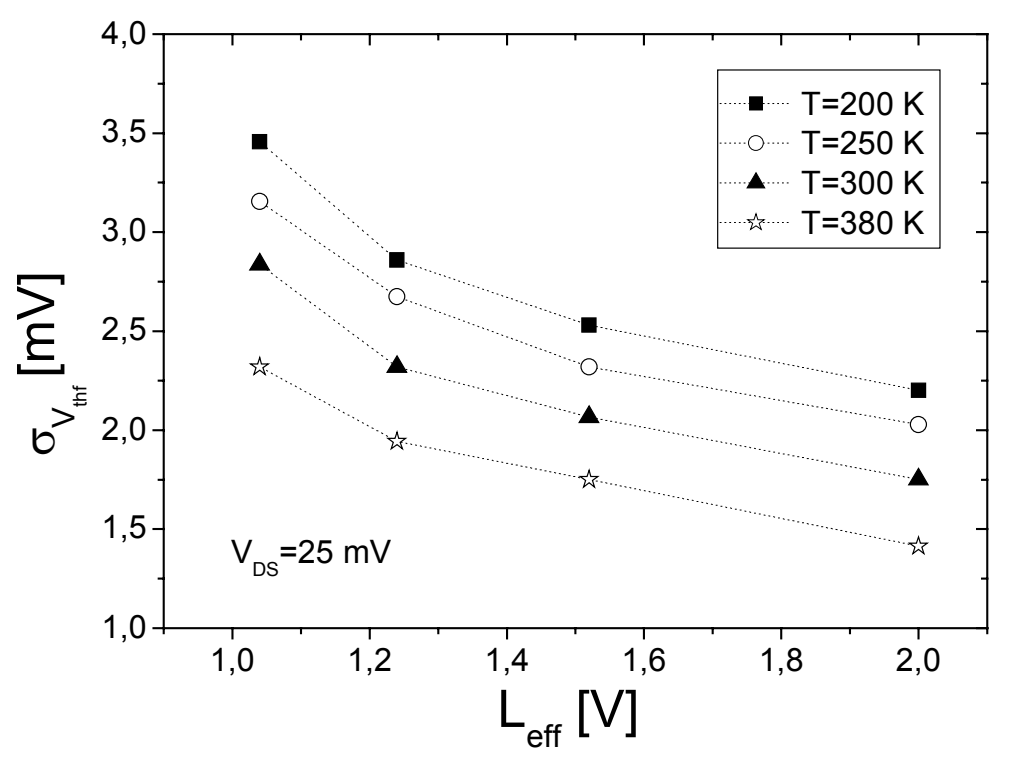

Figura 48 - Desvio padrão da tensão de limiar em função do comprimento efetivo de canal medido em diferentes temperaturas.

De acordo com as eqs. (71) e (72), a variação da tensão de limiar é dependente das espessuras das camadas de óxido de porta e de silício e da concentração de dopantes no canal. Dentre estes, apenas a concentração de dopantes pode ser considerada dependente da temperatura, devido à sua ionização incompleta quando a temperatura é reduzida; neste caso, $\mathrm{N}_{\mathrm{A}}$ deve ser substituído por $\mathrm{N}_{\mathrm{A}}{ }^{-}$na eq. (72).

Como pode ser visto nas eqs. (10) e (11), o número de impurezas ionizadas $\left(N_{A}{ }^{-}\right.$ou $\left.N_{D}{ }^{+}\right)$depende não somente da temperatura, mas também da concentração de impurezas implantadas $\left(N_{A}\right.$ ou $\left.N_{D}\right)$. Com o objetivo de verificar a influência destes dois parâmetros na porcentagem de impurezas ionizadas, as equações apresentadas na seção 2.2 foram utilizadas para calcular a relação $N_{A}{ }^{-} / \mathrm{N}_{A}$ em função da temperatura, considerando diferentes valores de $\mathrm{N}_{\mathrm{A}}$ e impondo uma variação de $\pm 10 \%$. De posse destas curvas, foi calculado o desvio padrão entre elas. 0 
resultado obtido é apresentado na Figura 49. Esta curva mostra que, para uma dada variação da concentração $\mathrm{N}_{\mathrm{A}}$, resultante da fabricação dos dispositivos, a redução da temperatura aumenta a variação da porcentagem de impurezas ionizadas, aumentando a variação de $\mathrm{V}_{\text {thf }}$ (eq. (72)). Além disso, nota-se que esta variação diminui com a redução da concentração média de dopantes.

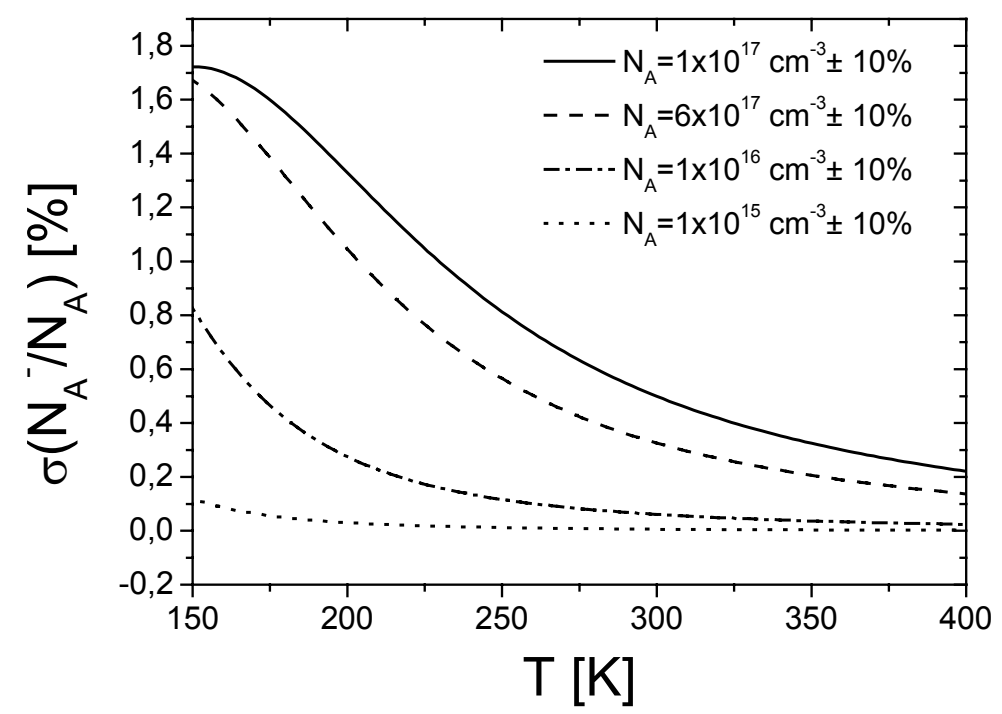

Figura 49 - Desvio padrão da porcentagem de impurezas ionizadas em função da temperatura, calculados a partir das equações apresentadas na seção 2.2, considerando diferentes concentrações de dopantes, $N_{A}$, e impondo variação de $\pm 10 \%$.

$\mathrm{Na}$ Figura 50 são apresentadas as curvas $\sigma_{\mathrm{V}_{\text {thf }}} / \mathrm{V}_{\text {thf,mean }}$ em função do comprimento de canal para as temperaturas estudadas. Pode-se notar que, ao dividir o desvio padrão pelo valor médio da tensão de limiar, as curvas tornam-se bastante próximas. Tal resultado mostra que o aumento de $\mathrm{V}_{\text {thf,mean }}$ causado pela redução da temperatura e conseqüente elevação do potencial de Fermi é suficiente para compensar a piora no casamento de $\mathrm{V}_{\text {thf }}$. 


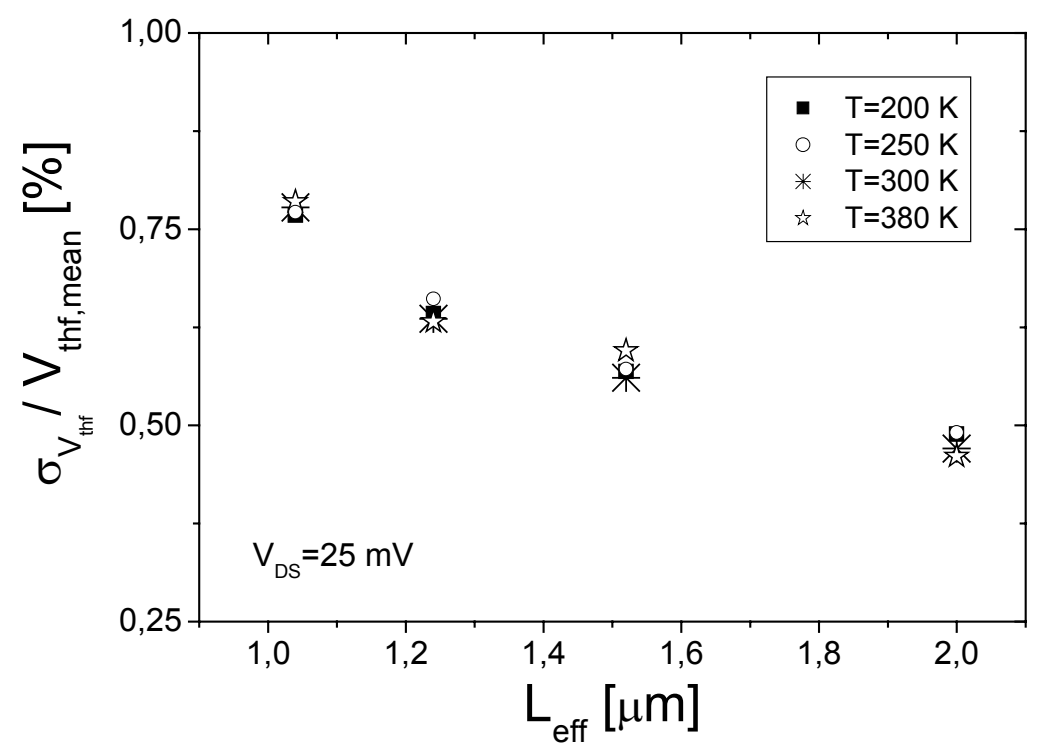

Figura $50-\sigma_{V_{\text {thf }}} / \bigvee_{\text {thf,mean }}$ em função do comprimento efetivo de canal, medido para diferentes temperaturas.

A Figura 51 apresenta a variação da corrente de dreno em função da corrente de dreno média normalizada, para transistores SOI convencionais e GC SOI com comprimento efetivo de canal médio de 1,52 $\mu \mathrm{m}$ ( $\left.\mathrm{L}_{\mathrm{LD}} / \mathrm{L}=0,24\right)$, medidos em saturação, com $V_{D S}=1,5 \mathrm{~V}$, em diferentes temperaturas. As curvas apresentadas permitem observar um aumento do descasamento da corrente de dreno com a redução da temperatura, além de uma degradação adicional provocada pela utilização da estrutura de canal gradual em inversão fraca.

De acordo com a ref. [129], o descasamento da corrente de dreno em inversão fraca é dominado pelo descasamento da tensão de limiar:

$$
\frac{\Delta \mathrm{I}_{\mathrm{DS}}}{\mathrm{I}_{\mathrm{DS}}}=-\frac{\mathrm{g}_{\mathrm{m}}}{\mathrm{I}_{\mathrm{DS}}} \Delta \mathrm{V}_{\mathrm{thf}}
$$

Sendo a relação $\mathrm{g}_{\mathrm{m}} / \mathrm{l}_{\mathrm{DS}}$ uma característica dependente apenas da tecnologia e da temperatura, não foram observadas diferenças entre as curvas dos transistores convencionais e de canal gradual. Assim, o aumento do descasamento da corrente de dreno com a redução da temperatura e utilização da estrutura $\mathrm{GC}$, em inversão fraca, depende exclusivamente do descasamento em $V_{\text {thf }}$, apresentando o mesmo comportamento deste parâmetro. À medida que o nível de inversão dos dispositivos 
aumenta, verifica-se a melhora da relação $\sigma \mathrm{I}_{\mathrm{DS}} / \mathrm{I}_{\mathrm{DS} \text {,mean }}$ em todas as temperaturas, como observado anteriormente em temperatura ambiente.

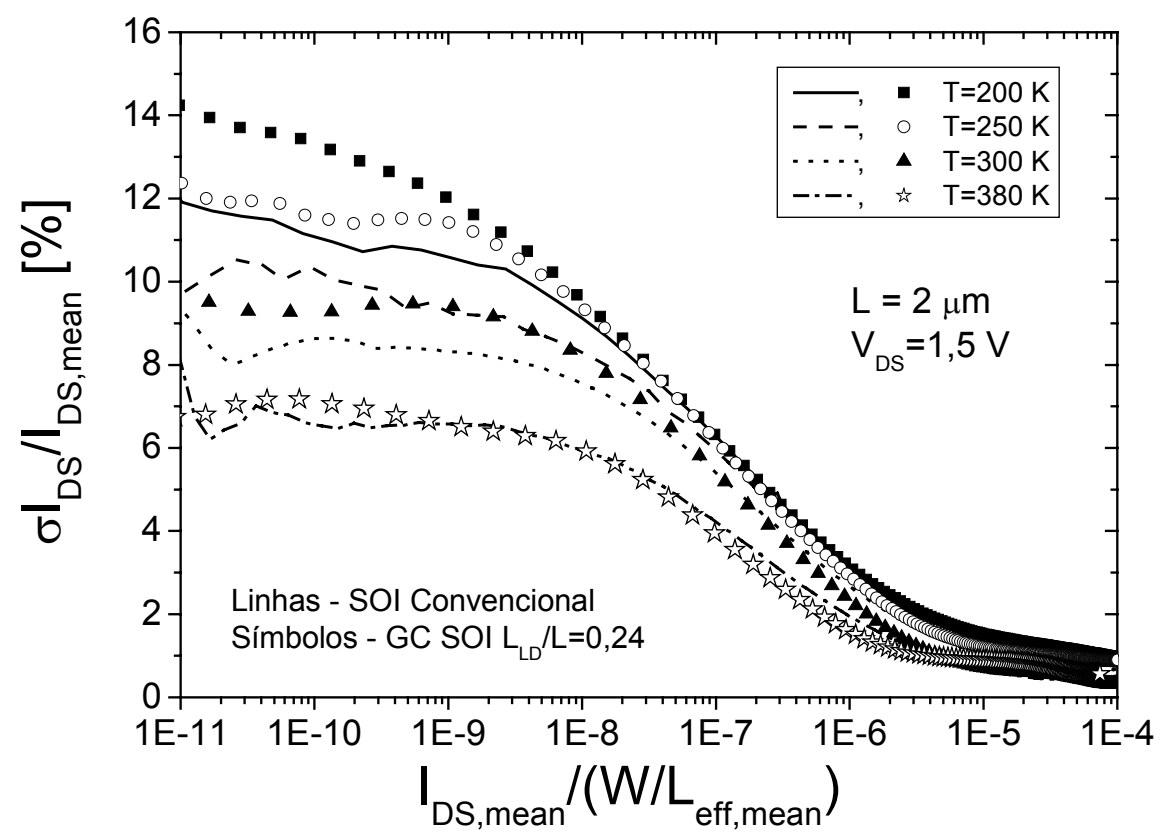

Figura 51 - Variação da corrente de dreno em função da corrente de dreno normalizada, medida para transistores SOI convencional e GC SOI com $L_{L D} / L=0,24$ em saturação $\left(V_{D S}=1,5 \mathrm{~V}\right)$.

SOUZA, M. de ; FLANDRE, D.; PAVANELLO, M. A. Analysis of Matching in GradedChannel SOI MOSFETS. Microelectronics Technology and Devices - SBMicro 2007, v. 9. p. 323-332, 2007.

SOUZA, M. de; FLANDRE, D.; PAVANELLO, M. A. Study of Matching Properties of Graded-Channel SOI MOSFETs. JICS. Journal of Integrated Circuits and Systems, v. 3, p. 69-75, 2008. 


\subsection{UTILIZAÇÃO DE TRANSISTORES GC SOI COMO AMPLIFICADOR DRENO COMUM}

Em várias aplicações analógicas, tais como no caso de células APS (active pixel sensor) em sensores de imagens implementados em tecnologia $\mathrm{CMOS}^{130,131}$, um estágio é necessário para o casamento de impedâncias entre entrada e saída. Idealmente, este conversor de impedâncias deve ter ganho unitário, impedância de entrada infinita e impedância de saída nula ${ }^{121,132}$. A implementação clássica de um estágio de ganho unitário é o amplificador dreno comum, mais comumente denominado seguidor de fonte ${ }^{132}$. Este consiste em um transistor em configuração de dreno comum, com entrada na porta e saída no terminal de fonte, polarizado por uma fonte de corrente constante, como apresentado na Figura 52. Em tecnologia CMOS convencional, o substrato pode ser aterrado ou conectado à fonte, enquanto em FD SOI CMOS, o terminal de substrato deve ser aterrado.

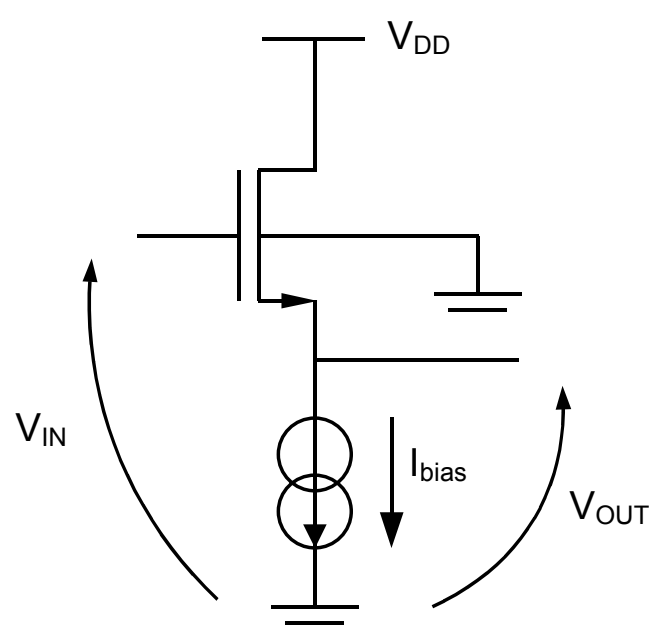

Figura 52 - Transistor configurado como amplificador dreno comum ou seguidor de fonte.

Apesar de sua simplicidade, o seguidor de fonte apresenta bom desempenho, com alta impedância de entrada, uma vez que o sinal de entrada é aplicado à porta do transistor MOS, e baixa impedância de saída ${ }^{121,132}$. Entretanto, sofre de efeitos tais como efeito de corpo, responsável pela alteração da tensão de limiar, e modulação do comprimento de canal, degradando a condutância de dreno. Estes 
efeitos são responsáveis pela distorção harmônica das características de saída do seguidor de fonte e fazem com que seu ganho seja menor do que a unidade.

\subsubsection{Análise experimental em temperatura ambiente ${ }^{133,134}$}

\subsubsection{Ganho de tensão}

Transistores GC SOI e SOI convencional foram medidos na configuração de amplificador dreno comum, como apresentado na Figura 52. As curvas características do seguidor de fonte foram obtidas com tensão de alimentação, $V_{\mathrm{DD}}$, de 2,5 V, e as curvas $V_{\text {Out }}$ em função de $V_{\mathbb{I N}}$ foram obtidas variando a tensão de entrada, $V_{\mathbb{I N}}$, que coincide com a tensão de porta do transistor, e medindo a tensão de saída, $V_{\text {OUt, }}$ no terminal de fonte. Com o intuito de avaliar a influência do nível de inversão sobre o desempenho do seguidor de fonte, foram realizadas medidas com diferentes correntes de polarização ( $\left.\mathrm{I}_{\text {bias }}\right)$ aplicadas à fonte. Além disso, foram realizadas medidas com a mesma corrente de polarização normalizada, $I_{n}=I_{\text {bias }} /\left(W / L_{\text {eff }}\right)$, para todos os transistores.

$\mathrm{Na}$ Figura 53 são apresentadas as curvas $\mathrm{V}_{\text {OUt }}$ em função de $\mathrm{V}_{\mathbb{I N}}$ obtidas em inversão fraca e forte, com corrente de polarização normalizada de 0,1 nA (A) e 4,0 $\mu \mathrm{A}(\mathrm{B})$, respectivamente. $\mathrm{O}$ nível de inversão, ou coeficiente $i_{C}$, foi definido através da relação $\mathrm{i}_{\mathrm{C}}=\mathrm{I}_{\mathrm{DS}} / \mathrm{I}_{\mathrm{S}}$, sendo $\mathrm{I}_{\mathrm{S}}$ a corrente de normalização, dada por $\mathrm{I}_{\mathrm{S}}=\mu_{\mathrm{n}} \mathrm{C}_{\text {oxf }}\left(\mathrm{V}_{\mathrm{T}}{ }^{2} / 2\right)\left(\mathrm{W} / \mathrm{L}_{\text {eff }}\right){ }^{135}$; sendo a mobilidade $\mu_{\mathrm{n}}$ estimada através do modelo analítico apresentado anteriormente. Para valores de $i_{C}$ menores ou iguais a 1 , o dispositivo opera em inversão fraca; para valores iguais ou maiores que 100, o dispositivo opera em inversão forte, enquanto valores intermediários de $i_{C}$ caracterizam a operação em inversão moderada. Para as correntes de polarização escolhidas, os valores de $i_{C}$ são da ordem de $4,5 \times 10^{-3}$ e 180 , assegurando a operação em inversão fraca e forte, respectivamente. 
O ganho de tensão do seguidor de fonte, $A_{V}$, foi obtido através da derivada numérica de $\mathrm{V}_{\text {OUT }}$ com relação a $\mathrm{V}_{\mathrm{IN}}\left(\mathrm{A}_{\mathrm{V}}=\delta \mathrm{V}_{\mathrm{OUT}} / \delta \mathrm{V}_{\mathrm{IN}}\right)$ e também é apresentado na Figura 53.
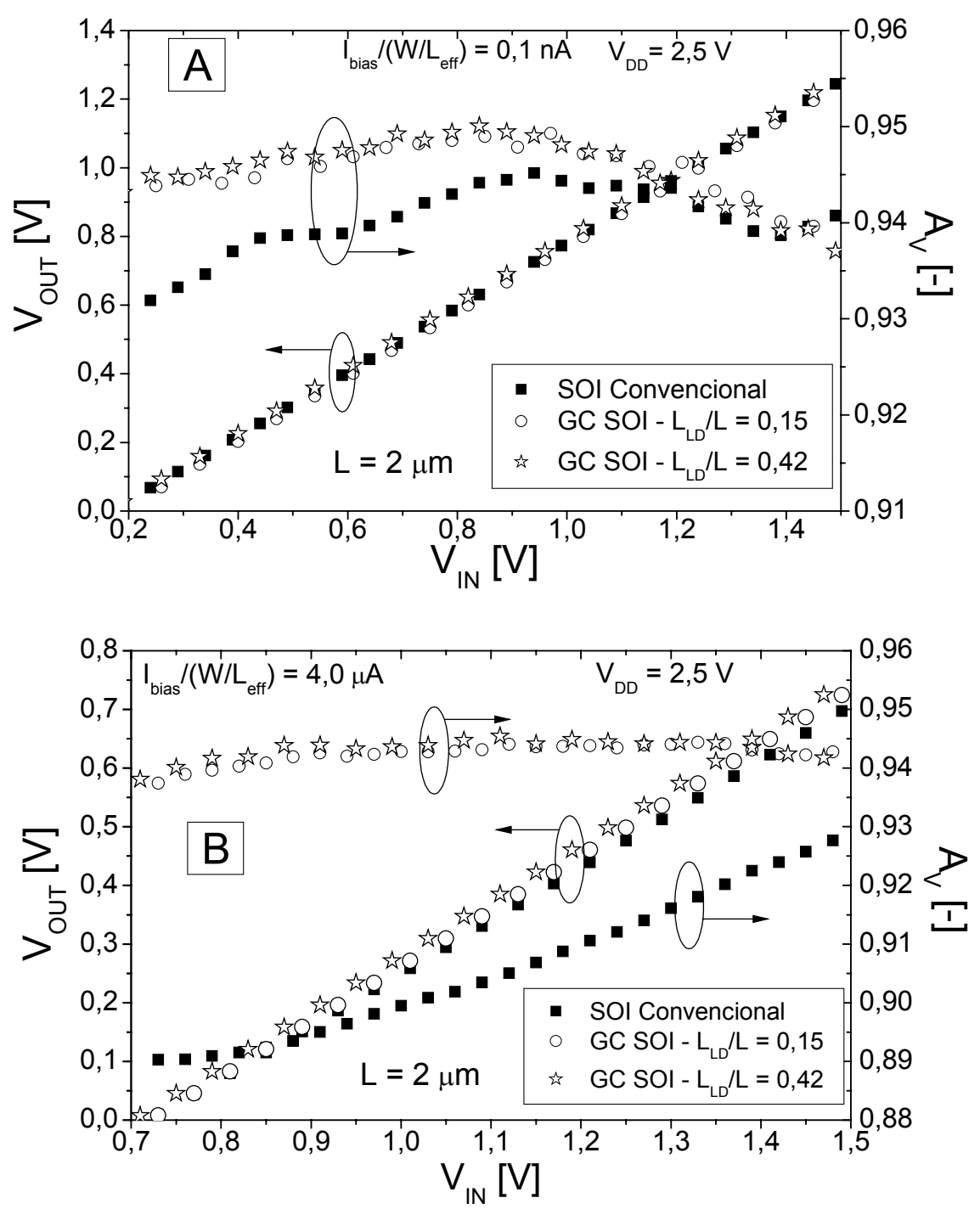

Figura 53 - Curvas $V_{\text {OUT }}$ e $A_{V}$ em função de $V_{\text {IN }}$ de seguidores de fonte implementados com transistores SOI convencional e de canal gradual, medidas em inversão fraca $(A)$ e forte $(B)$.

A partir dos resultados apresentados, pode-se notar que, apesar da proximidade entre as curvas $\mathrm{V}_{\text {OUT }}$ em função de $\mathrm{V}_{\mathrm{IN}}$ dos seguidores de fonte implementados com transistores convencional e de canal gradual, que pode ser explicada pela semelhança entre as tensões de limiar destes transistores e 
densidade de corrente, a utilização de transistores GC permite a obtenção de seguidores de fonte com ganho mais próximo à unidade do que o transistor convencional, independentemente da relação $\mathrm{L}_{\mathrm{LD}} / \mathrm{L}$.

Com o objetivo de relacionar esta melhora do ganho aos parâmetros de pequenos sinais dos transistores, a análise de pequenos sinais foi realizada, e o modelo equivalente é apresentado na Figura 54. Neste modelo, $g_{\mathrm{ms}}$ é a transcondutância de fonte $\left(g_{m s}=-d l_{D s} / d V_{S}\right)$ e $R$ é a resistência interna da fonte de corrente utilizada para a polarização do circuito.

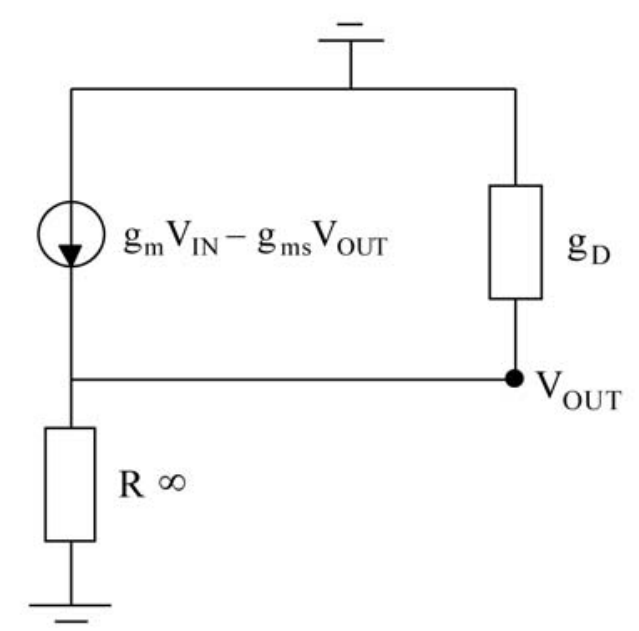

Figura 54 - Modelo equivalente de pequenos sinais para o seguidor de fonte.

A partir do modelo de pequenos sinais apresentado, tem-se:

$$
g_{\mathrm{m}} \mathrm{V}_{\mathrm{IN}}-g_{\mathrm{ms}} \mathrm{V}_{\text {OUT }}=\frac{\mathrm{V}_{\text {OUT }}}{\frac{1}{\left(\frac{1}{R}+g_{\mathrm{D}}\right)}}
$$

Sabendo que $\mathrm{g}_{\mathrm{ms}}=\mathrm{ng}_{\mathrm{m}}$, para dispositivos operando em saturação em todos os regimes de inversão ${ }^{136}$, e que $R$ é muito maior que $g_{D}$, o ganho, $A_{V}$, pode ser expresso por:

$$
A_{v}=\frac{V_{\text {OUT }}}{V_{\text {IN }}}=\frac{g_{m}}{n g_{m}+g_{D}}
$$

Através da eq. (77) pode-se notar que o limite teórico para o ganho do seguidor de fonte é igual a $1 / \mathrm{n}^{132}$, alcançado quando o valor de $g_{D}$ é desprezível 
face ao valor de $\mathrm{g}_{\mathrm{m}}$. O fator de corpo, $\mathrm{n}$, é um parâmetro dependente da tecnologia e pode-se considerar que sua variação seja a mesma em transistores convencionais e de canal gradual. A Tabela 8 apresenta os valores do fator de corpo extraídos através da inclinação de sublimiar das curvas $\mathrm{I}_{\mathrm{DS}}$ em função de $\mathrm{V}_{\mathrm{GF}}{ }^{51}$ dos transistores de canal gradual e convencional, obtidas com $\mathrm{V}_{\mathrm{DS}}=50 \mathrm{mV}$ e $1,5 \mathrm{~V}$. Pode-se notar que o fator de corpo é praticamente o mesmo para todos os dispositivos e valores de $V_{D S}$ estudados. Desta forma, a melhora no ganho, proporcionada pela utilização do transistor com canal gradual não está relacionada à variação do fator $n$ entre os dispositivos, mas sim à transcondutância e condutância de dreno.

Tabela 8 - Fator de corpo (n) extraído através da inclinação de sublimiar de curvas $I_{D S}$ em função de $V_{G F}$ experimentais, com $V_{D S}=50 \mathrm{mV}$ and $1,5 \mathrm{~V}$.

\begin{tabular}{ccc}
\hline Transistor & $\mathbf{n}\left(\mathbf{V}_{\mathbf{D S}}=\mathbf{5 0} \mathbf{~ m V}\right)$ & $\mathbf{n}\left(\mathbf{V}_{\mathbf{D S}}=\mathbf{1 , 5} \mathbf{~}\right)$ \\
\hline SOI Convencional & 1,101 & 1,106 \\
\hline GC SOI - $\mathrm{L}_{\mathrm{LD}} / \mathrm{L}=0,15$ & 1,099 & 1,099 \\
\hline GC SOI - $\mathrm{L}_{\mathrm{LD}} / \mathrm{L}=0,35$ & 1,098 & 1,099 \\
\hline GC SOI - $\mathrm{L}_{\mathrm{LD}} / \mathrm{L}=0,42$ & 1,104 & 1,106 \\
\hline
\end{tabular}

Desta forma, a fim de analisar a influência de $g_{m}$ e $g_{D}$ sobre o ganho do seguidor de fonte, as figuras abaixo apresentam os valores experimentais destes parâmetros, extraídos com $\mathrm{V}_{\mathrm{DD}}=2,5 \mathrm{~V}$ e $\mathrm{V}_{\mathrm{S}}=0 \mathrm{~V}$. Apesar destas condições de polarização serem um caso particular das curvas apresentadas na Figura 53, esta análise permite avaliar o comportamento do seguidor de fonte para diferentes transistores operando sob as mesmas condições de polarização. 


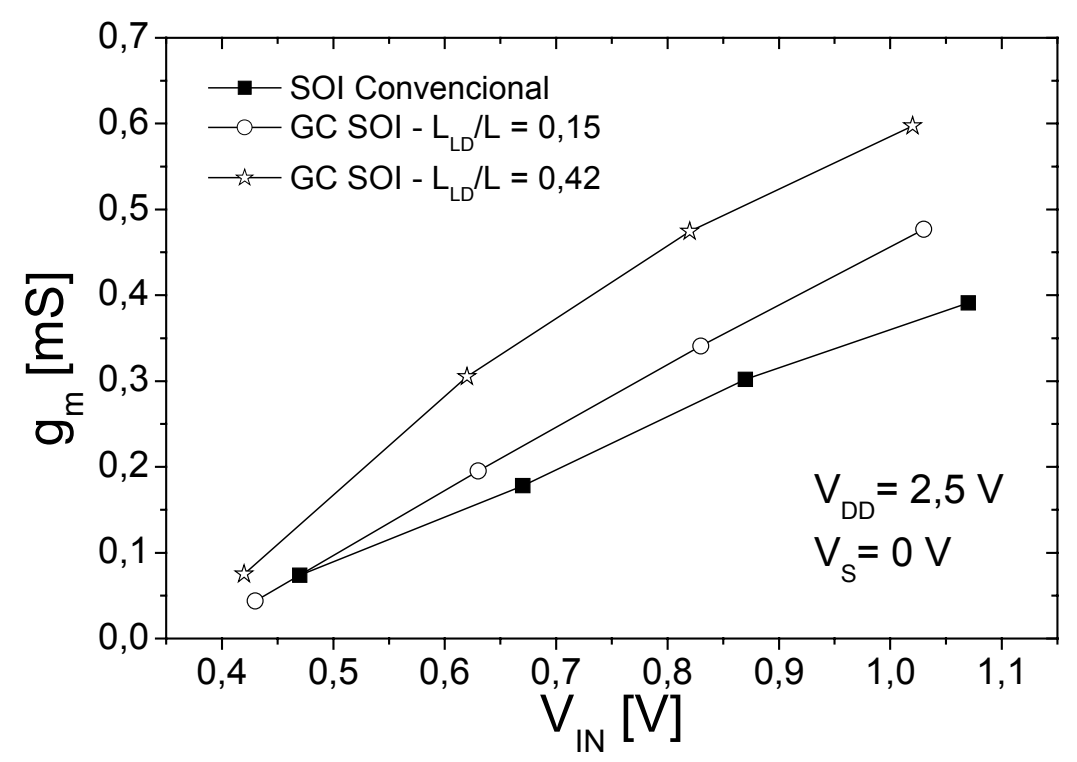

Figura 55 -Transcondutância em função da tensão de entrada, extraída a partir de curvas $I_{D S}$ em função de $V_{G F}$ experimentais, medidas com $V_{D D}=2,5 \mathrm{~V}$ e $V_{S}=0 \mathrm{~V}$.

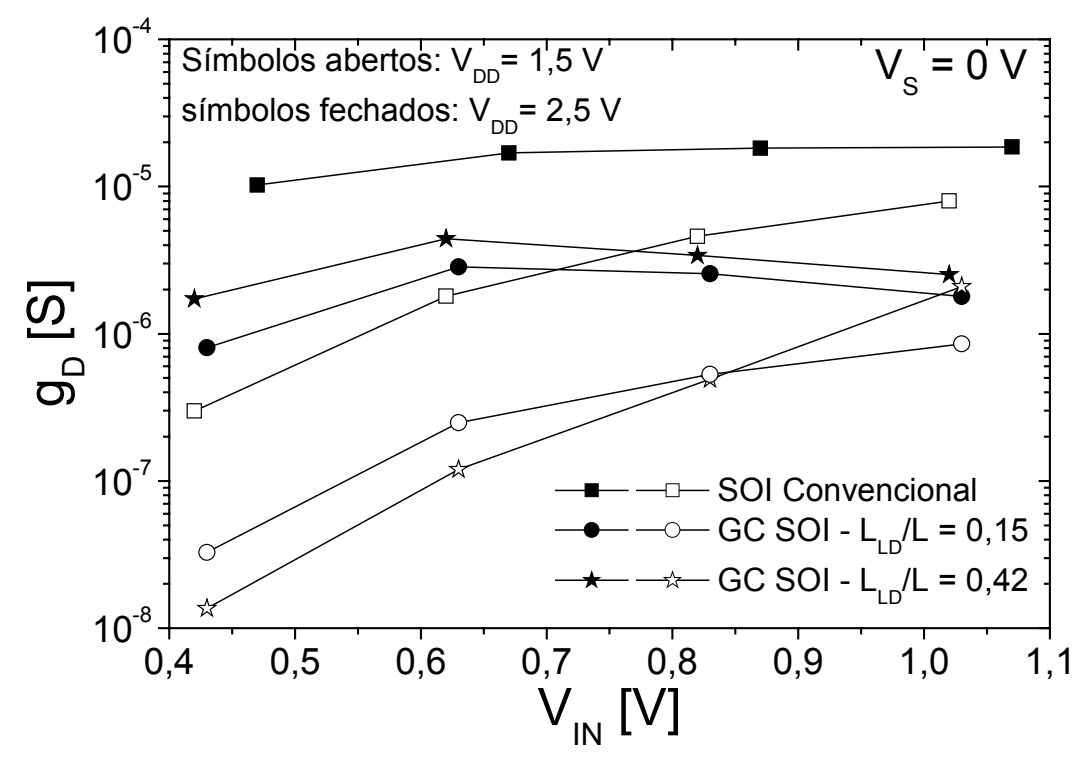

Figura 56 - Condutância de saída experimental extraída para $\mathrm{V}_{\mathrm{DD}}=1,5 \mathrm{~V}$ (símbolos abertos) e $2,5 \mathrm{~V}$ (símbolos fechados), com $\mathrm{V}_{\mathrm{S}}=0 \mathrm{~V}$.

Uma vez que o comprimento efetivo de canal diminui com o aumento da relação $L_{L D} / L$, pode-se notar que a transcondutância é maior nos transistores de canal gradual em comparação ao transistor SOI convencional. À medida que a tensão de entrada é reduzida, os dispositivos se aproximam da inversão fraca, 
causando a redução da transcondutância, cujos valores tornam-se da mesma ordem de grandeza dos valores da condutância de saída, degradando o ganho.

Além disso, a presença de uma região fracamente dopada próxima ao dreno reduz a variação do potencial que alcança a região fortemente dopada do canal $\left(\mathrm{V}_{\mathrm{D}, \mathrm{HD}}\right)^{137}$, promovendo a redução da modulação do comprimento de canal causada pela polarização de dreno. Esta redução é responsável pelos menores valores de $g_{D}$ apresentados por transistores GC, mesmo apresentando menor comprimento efetivo de canal do que o transistor convencional. Pode-se também notar que a condutância de dreno do transistor de canal gradual com $L_{L D} / L=0,42$ sofre uma pequena degradação em comparação ao de $L_{L D} / L=0,15$, embora ainda apresente vantagem em relação ao transistor convencional. Tal fato pode ser atribuído à modulação do comprimento de canal da região fortemente dopada, à medida que a relação $L_{L D} / L$ aumenta.

Assim, os maiores valores de $g_{m}$ e menores valores de $g_{D}$ apresentados pelos transistores de canal gradual são responsáveis pelo aumento do ganho de tensão observado (eq. (77)).

Considerando-se a configuração do seguidor de fonte, apresentada na Figura 52 , pode-se notar que a redução da tensão de entrada $V_{\mathbb{I N}}$, e conseqüente redução de $V_{\text {OUT }}\left(V_{S}\right)$, promove o aumento da queda de tensão entre o dreno e a fonte do transistor $\left(V_{D S}=V_{D D}-V_{O U T}\right)$, uma vez que a tensão $V_{D D}$ é mantida constante. Como apresentado na Figura 56, o aumento de $V_{D S}$ provoca a degradação de $g_{D}$, reduzindo o ganho para menores valores de $\mathrm{V}_{\mathrm{IN}}$. No caso de transistores com canal gradual, que apresentam menor condutância de dreno, menor efeito de ionização por impacto e maior tensão de ruptura ${ }^{13}, g_{D}$ apresenta menores valores para uma excursão maior de $V_{D S}$ que o transistor convencional, justificando, assim, o fato do ganho de seguidores de fonte implementados com transistores GC permanecer praticamente constante durante toda a excursão da tensão de entrada. Por outro lado, o seguidor de fonte implementado com transistor SOI convencional apresenta uma redução significativa do ganho, principalmente em inversão forte. Além disso, os maiores valores de $\mathrm{g}_{\mathrm{m}}$ apresentados pelos transistores de canal gradual, fazem com que uma maior degradação de g g $_{\mathrm{D}}$ seja necessária para causar a degradação de Av.

A partir das curvas da corrente de dreno em função da tensão de porta e da tensão de fonte medidas para os transistores em estudo, foram extraídos os valores 
de $g_{m}$ e $g_{m s}$, respectivamente, em saturação, com o objetivo de extrair o fator $1 / n$, definido como $\mathrm{g}_{\mathrm{m}} / \mathrm{g}_{\mathrm{ms}}$. Os resultados obtidos são apresentados na Figura 57 , em função da tensão $V_{\mathbb{I N}}\left(V_{G F}\right)$. Utilizando os resultados de $1 / n$ e os valores de $g_{m}$ e $g_{D}$ apresentados nas Figuras 55 e 56, o ganho foi calculado através da eq. (77). Os valores obtidos são apresentados na mesma figura.

A análise dos resultados apresentados permite verificar que seguidores de fonte implementados com transistores de canal gradual apresentam ganho bastante próximo do limite teórico em toda a excursão de $V_{\mathbb{I N}}$ estudada. No caso do transistor convencional, $A_{V}$ apresenta uma degradação significativa, principalmente para menores valores de $V_{\mathbb{I N}}$, para os quais chega a $11 \%$. Vale ressaltar que estes resultados concordam com aqueles obtidos experimentalmente, apresentados na Figura 53.

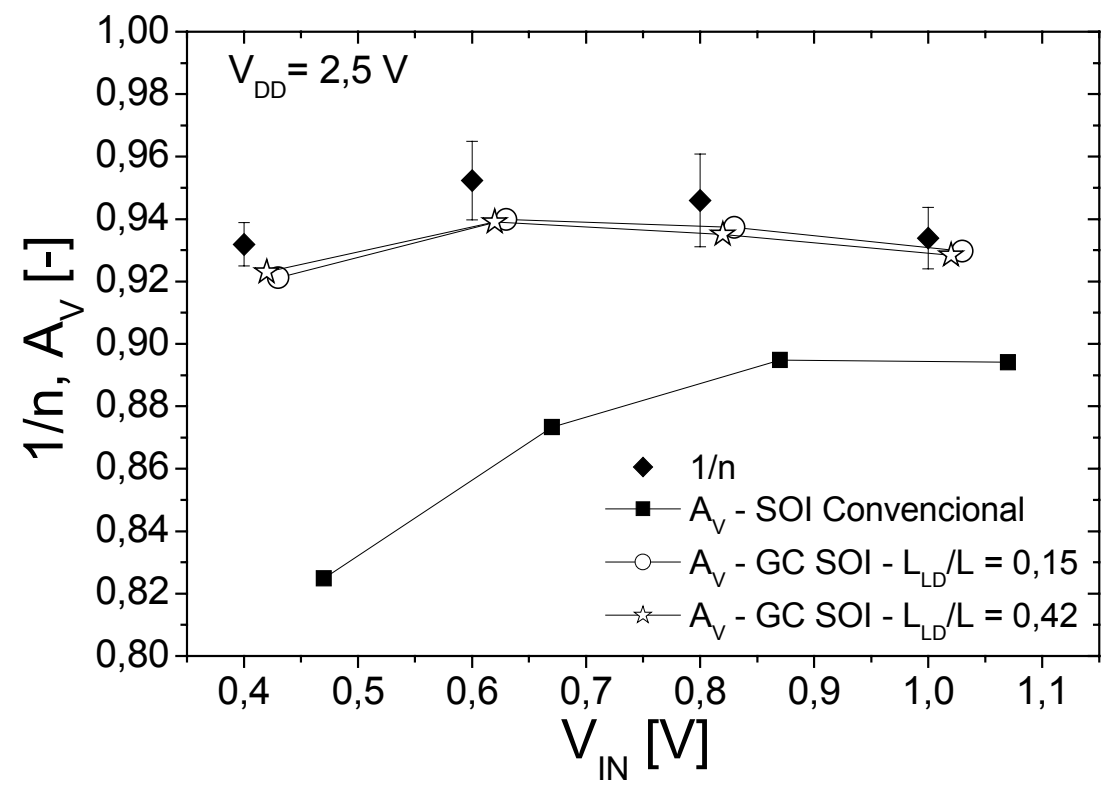

Figura 57 - Fator 1/n obtido experimentalmente e $A_{\vee}$ calculado, em função da tensão de entrada com $V_{D D}=1,5 \mathrm{~V}$.

\subsubsection{Linearidade}

Sabe-se que seguidores de fonte apresentam curvas $V_{\text {OUt }}$ em função de $V_{\text {IN }}$ não-lineares, devido à dependência não linear da tensão de limiar com a tensão $V_{S} e$ 
à dependência de $g_{D}$ com a queda de tensão entre fonte e dreno do transistor ${ }^{132}$. Essas não-linearidades induzem, na tensão de saída, componentes harmônicas de freqüências múltiplas às do sinal aplicado à sua entrada.

A fim de avaliar a influência da utilização da estrutura de canal gradual sobre a linearidade de seguidores de fonte, a distorção harmônica total (THD) foi extraída aplicando o método da função integral (IFM) ${ }^{82}$ às curvas $V_{\text {OUt }}$ em função de $V_{\text {IN }}$. Nesta configuração, o método considera, como sinal de entrada, uma tensão de polarização que coincide com $\mathrm{V}_{\mathrm{IN}}$, associada a um sinal senoidal de amplitude $\mathrm{Va}$. Os resultados extraídos em inversão fraca e forte são apresentados na Figura 58, considerando $\mathrm{Va}=50 \mathrm{mV}$.
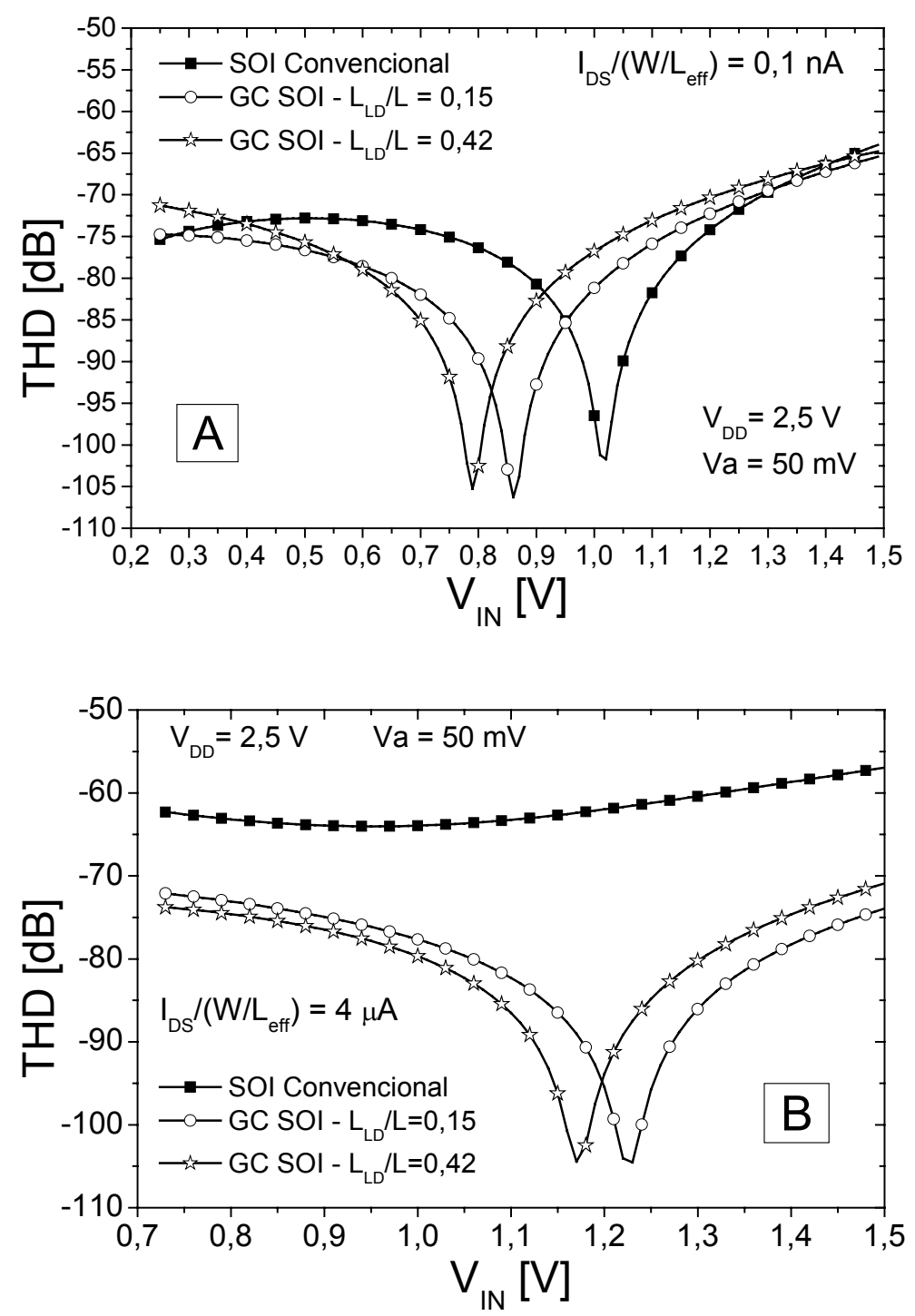

Figura 58 - Distorção harmônica total (THD) em função de $V_{\mathbb{I N}}$, for seguidores de fonte polarizados em inversão fraca $(A)$ e forte $(B)$, com $V_{D D}=2,5 \mathrm{~V}$ e $\mathrm{Va}=50 \mathrm{mV}$. 
Em inversão fraca observa-se que THD tende a apresentar nível de distorção semelhante para todos os dispositivos, a menos do deslocamento do ponto de distorção mínima, que está relacionado ao aumento da tensão de saturação que ocorre à medida que o comprimento LLD é aumentado. Este resultado indica que a utilização de transistores de canal gradual como seguidor de fonte é particularmente interessante para aplicações com baixa tensão de alimentação, uma vez que proporciona maior linearidade para menores níveis de tensão aplicados à entrada do que o transistor convencional.

A melhora na linearidade, promovida pela utilização de transistores de canal gradual, torna-se mais evidente com o aumento do nível de inversão. Os resultados obtidos indicam que, sob estas condições de polarização, em temperatura ambiente, transistores de canal gradual são capazes de melhorar em mais de $10 \mathrm{~dB}$ a linearidade do seguidor de fonte, em comparação com o transistor convencional.

De acordo com a ref. [138], a distorção harmônica de segunda ordem, que é dominante em transistores MOS operando em saturação, como no caso do seguidor de fonte, em inversão forte, é dada por

$$
H D 2 \approx T H D \approx\left(\frac{g_{D}}{2 \cdot g_{m}}\right)^{2} \cdot \frac{V_{i p}}{V_{G S}-V_{T}}
$$

onde $V_{\text {ip }}$ é a tensão de entrada de pico ${ }^{138}$.

Para dispositivos sob as mesmas condições de polarização, e considerando que transistores de canal gradual apresentam tensão de limiar semelhante à de transistores convencionais com mesmo comprimento total de canal, a redução de $g_{D}$ já seria condição suficiente para justificar a redução da distorção harmônica de seguidores de fonte causada pela utilização de transistores com canal gradual (eq. (78)). Entretanto, além da redução de g maior $\mathrm{g}_{\mathrm{m}}$, devido à redução do comprimento efetivo de canal, contribuindo para uma melhora ainda maior na distorção harmônica total do seguidor de fonte.

Além disso, pode-se observar que seguidores de fonte implementados com transistores de canal gradual apresentam maior vantagem, do ponto de vista da linearidade, quando polarizados em inversão forte, uma vez que a melhora na sua condutância de saída, em relação a um transistor uniformemente dopado, aumenta com o nível de inversão ${ }^{99}$. 


\subsubsection{Influência da ionização por impacto no ganho de tensão de seguidores de fonte}

A análise dos resultados experimentais sugere que o fato do ganho de tensão de seguidores de fonte implementados com transistores de canal gradual permanecer praticamente constante com a variação de $V_{\mathbb{I N}}$ pode estar relacionado à melhora da condutância de saída, proporcionada pela redução da ionização por impacto nos transistores de canal gradual. Com o intuito de verificar esta hipótese, simulações numéricas bidimensionais foram realizadas. Um arquivo de exemplo das simulações realizadas é apresentado no Apêndice C.

Seguidores de fonte foram simulados considerando transistores com as mesmas dimensões e parâmetros tecnológicos dos dispositivos caracterizados, largura de canal de $1 \mu \mathrm{m}$ e $\mathrm{L}_{\mathrm{LD}} / \mathrm{L}=0,2 ; 0,4$ e 0,5. A concentração de dopantes utilizada foi de $5,7 \times 10^{16} \mathrm{~cm}^{-3}$ a fim de ajustar a tensão de limiar simulada à obtida experimentalmente. Os parâmetros dos modelos de mobilidade e ionização por impacto foram otimizados para aproximar os resultados simulados dos experimentais. Foram utilizadas as mesmas condições de polarização aplicadas aos dispositivos experimentais.

A fim de verificar a influência da ionização por impacto no ganho de tensão dos seguidores de fonte, foram realizadas simulações de transistores com $L=2 \mu \mathrm{m}$ e diferentes relações $L_{L D} / L$, com e sem o modelo que considera este efeito (SELB). $A$ comparação entre os resultados obtidos é apresentada na Figura 59 em inversão fraca $(A)$ e forte $(B)$, com $I_{\text {bias }} /\left(W / L_{\text {eff }}\right)=0,1 \mathrm{nA}$ e $4,0 \mu A$, respectivamente, com $\mathrm{V}_{\mathrm{DD}}=2,5 \mathrm{~V}$. 

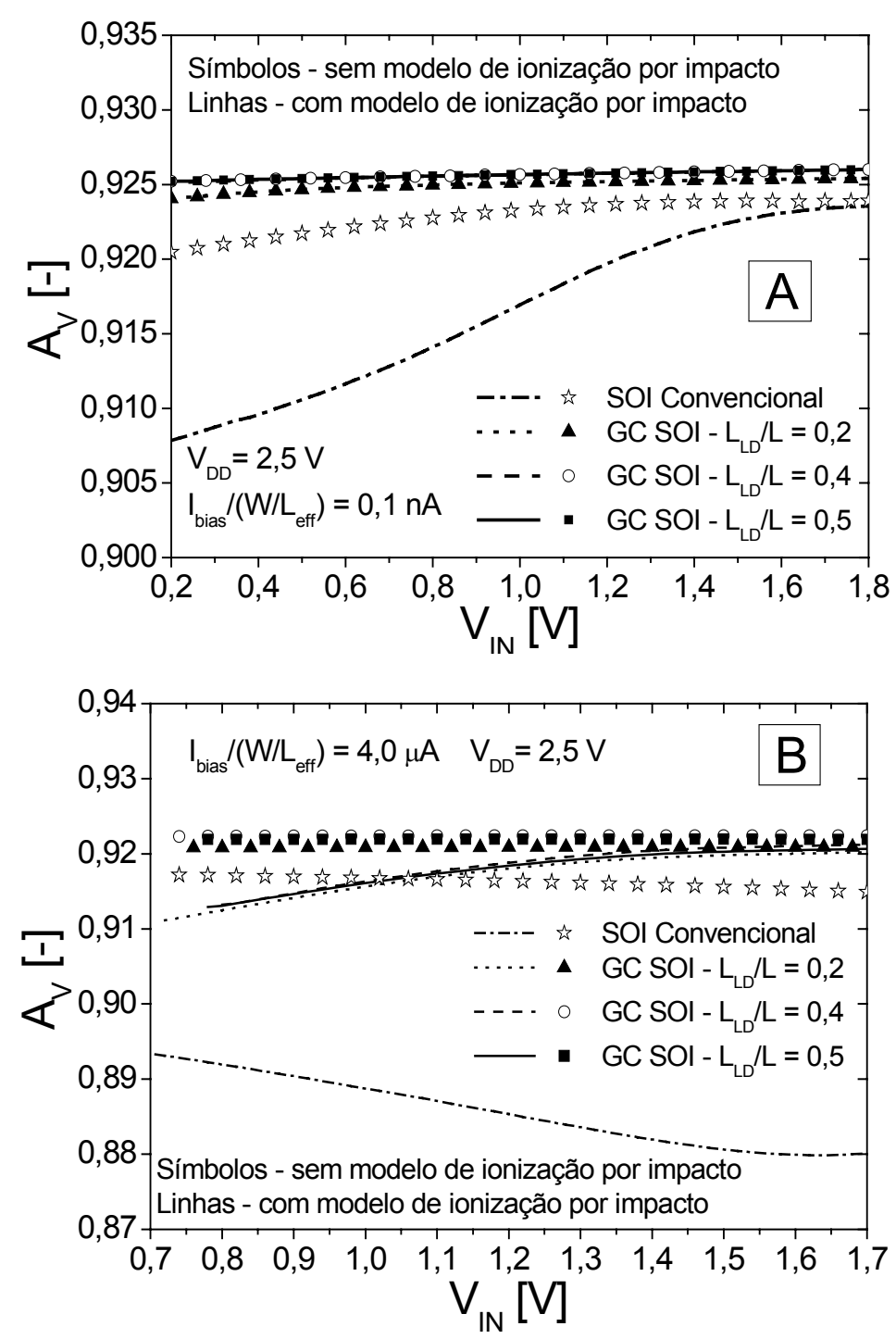

Figura 59 - Ganho de tensão em função da tensão de entrada simulado para seguidores de fonte com transistores convencional e de canal gradual $(\mathrm{L}=2 \mu \mathrm{m})$, com (linhas) e sem (símbolos) o modelo de ionização por impacto, em inversão fraca (A) e forte $(B)$, com $V_{D D}=2,5 \mathrm{~V}$.

Como pode ser visto nas curvas apresentadas, a inclusão do modelo de ionização por impacto nas simulações causa a redução do ganho à medida que a queda de tensão $V_{D S}$ aumenta (redução de $V_{I N}$ ). Quando o efeito de ionização por impacto é desprezado, o ganho dos seguidores de fonte permanece praticamente constante para todos os dispositivos simulados, confirmando a hipótese levantada a partir dos dados experimentais. Ainda em concordância com os resultados experimentais, a redução do ganho causado pela ocorrência de ionização por impacto torna-se maior com o aumento do regime de inversão, devido à maior degradação da condutância de saída. 
Os resultados obtidos permitem ainda observar que, mesmo na ausência de ionização por impacto, seguidores de fonte implementados com transistores de canal gradual apresentam $A_{V}$ ligeiramente maior do que aquele implementado com transistor convencional, devido à maior transcondutância e menor condutância de dreno dos transistores GC SOI.

\subsubsection{Influência da redução do comprimento de canal sobre o desempenho de seguidores de fonte ${ }^{139}$}

Como demonstrado nas seções anteriores, a melhora do ganho de tensão e linearidade de seguidores de fonte utilizando transistores GC em relação ao transistor convencional está relacionada à melhora na transcondutância e na condutância de dreno promovida por esta estrutura. No caso da condutância de dreno, a melhora promovida pela estrutura de canal gradual está associada à redução dos efeitos de ionização por impacto e de modulação do comprimento de canal em comparação com um transistor convencional de mesmo comprimento total de máscara.

Estes efeitos tendem a tornar-se mais significativos à medida que o comprimento de canal do transistor é reduzido. Desta forma, pode-se esperar que, para seguidores de fonte implementados com transistores de canal curto, o uso de transistores GC seja mais efetivo para a melhora de seu desempenho e que o aumento do comprimento de canal faça com que as vantagens apresentadas sejam menos significativas.

\subsubsection{Resultados experimentais}

Com o objetivo de avaliar a influência da redução do comprimento de canal no desempenho de seguidores de fonte implementados com transistores SOI convencional e GC, inicialmente foram caracterizados dispositivos com largura de canal de $20 \mu \mathrm{m}$, comprimento total de canal de $2 \mu \mathrm{m}, 1 \mu \mathrm{m}$ e 0,75 $\mu \mathrm{m}$, com diferentes relações $L_{L D} / L$. Os transistores foram submetidos a testes experimentais na mesma configuração e condições de polarização apresentadas anteriormente, 
utilizando o Sistema de Caracterização de Semicondutores Keithley 4200 para a obtenção das curvas $\mathrm{V}_{\mathrm{OUT}}$ em função de $\mathrm{V}_{\mathrm{IN}}$.

A Figura 60 apresenta as curvas $A_{V}$ em função de $V_{\mathbb{I N}}$ obtidas em inversão fraca e forte, com $I_{\text {bias }} /\left(W / L_{\text {eff }}\right)=0,1 \mathrm{nA}$ e 4,0 $\mu \mathrm{A}$, para seguidores de fonte implementados com transistores convencionais e de canal gradual com diferentes comprimentos de canal e relações $L_{L D} / L$. Na Figura também é indicado o comprimento efetivo de canal de cada transistor. Conforme previsto, o ganho de tensão sofre uma maior degradação com a redução de $V_{\mathbb{N}}$ à medida que 0 comprimento de canal é reduzido, devido à degradação de $g_{D}$. Além disso, a redução de $L$ faz com que o máximo ganho atingido (obtidos para maiores valores de $V_{I N}$ ) seja menor, tanto para transistores SOI convencionais como de canal gradual.

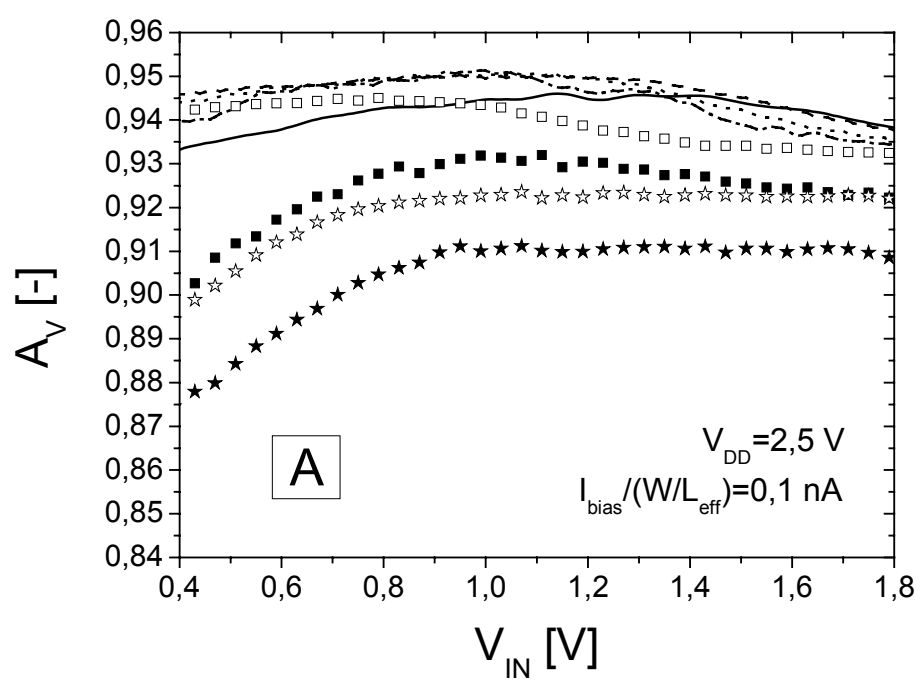

Linhas $-\mathrm{L}=2,00 \mu \mathrm{m}$

Quadrados - $L=1,00 \mu \mathrm{m}$

Estrelas $-\mathrm{L}=0,75 \mu \mathrm{m}$

Convencional, $\mathrm{L}_{\text {eff }}=2,00 \mu \mathrm{m}$

- - - $\mathrm{L}_{\mathrm{LD}} / \mathrm{L}=0,50, \mathrm{~L}_{\text {eff }}=1,00 \mu \mathrm{m}$

$\cdots \mathrm{L}_{\mathrm{LD}} / \mathrm{L}=0,62, \mathrm{~L}_{\text {eff }}=0,76 \mu \mathrm{m}$

$\cdots-\cdots L_{L D} / L=0,72, L_{\text {eff }}=0,56 \mu \mathrm{m}$

- Convencional, $\mathrm{L}_{\text {eff }}=1,00 \mu \mathrm{m}$

- $\mathrm{L}_{\mathrm{LD}} / \mathrm{L}=0,47, \mathrm{~L}_{\text {eff }}=0,53 \mu \mathrm{m}$

* Convencional, $\mathrm{L}_{\text {eff }}=0,75 \mu \mathrm{m}$

\& $\mathrm{L}_{\mathrm{LD}} / \mathrm{L}=0,25, \mathrm{~L}_{\text {eff }}=0,56 \mu \mathrm{m}$

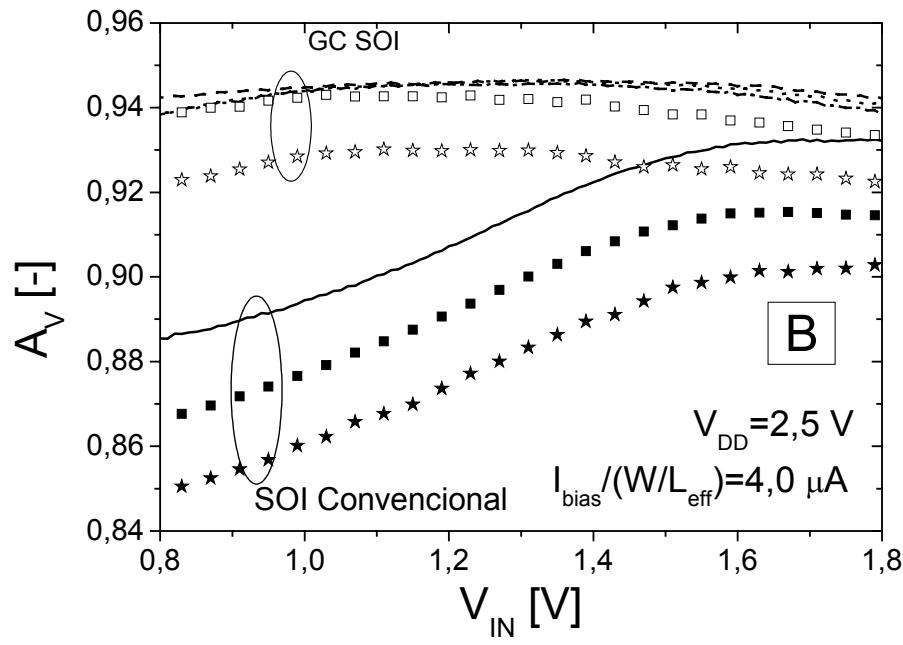

Linhas $-\mathrm{L}=2,00 \mu \mathrm{m}$

Quadrados - $\mathrm{L}=1,00 \mu \mathrm{m}$

Estrelas $-\mathrm{L}=0,75 \mu \mathrm{m}$

- Convencional, $\mathrm{L}_{\text {eff }}=2,00 \mu \mathrm{m}$

- - - $L_{L D} / L=0,50, L_{\text {eff }}=1,00 \mu m$

… $L_{L D} / L=0,62, L_{\text {eff }}=0,76 \mu \mathrm{m}$

-.-- $L_{L D} / L=0,72, L_{\text {eff }}=0,56 \mu \mathrm{m}$

- Convencional, $\mathrm{L}_{\text {eff }}=1,00 \mu \mathrm{m}$

- $\mathrm{L}_{\mathrm{LD}} / \mathrm{L}=0,47, \mathrm{~L}_{\text {eff }}=0,53 \mu \mathrm{m}$

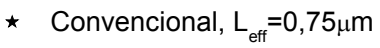

\& $\mathrm{L}_{\mathrm{LD}} / \mathrm{L}=0,25, \mathrm{~L}_{\text {eff }}=0,56 \mu \mathrm{m}$

Figura 60 - Ganho experimental de seguidores de fonte implementados com transistores SOI convencionais e de canal gradual com diferentes comprimentos de canal, polarizados em inversão fraca $(A)$ e forte $(B)$, com $I_{\text {bias }} /\left(W / L_{\text {eff }}\right)=0,1 \mathrm{nA}$ e $4,0 \mu \mathrm{A}$, respectivamente, e $\mathrm{V}_{D D}=2,5 \mathrm{~V}$. 
Como discutido anteriormente, o limite teórico para o ganho é dado por $1 / \mathrm{n}$. No caso de transistores de canal curto, o fator de corpo sofre um aumento, conforme visto na Tabela 9, a qual apresenta os valores do fator de corpo extraídos a partir da inclinação de sublimiar das curvas $\mathrm{I}_{\mathrm{DS}}$ em função de $\mathrm{V}_{\mathrm{GF}}{ }^{51}$ medidas com $\mathrm{V}_{\mathrm{DS}}=50 \mathrm{mV}$ e 1,5 V. Observa-se que, além do aumento de $\mathrm{n}$ com a redução do comprimento total de canal, responsável pela diminuição do limite teórico do ganho, para um dado $\mathrm{L}$, o fator $\mathrm{n}$ é praticamente o mesmo para transistores de canal gradual e convencional, indicando que as diferenças verificadas nas curvas do ganho não dependem deste parâmetro.

Tabela 9 - Fator de corpo (n) extraído através da inclinação de sublimiar de curvas $\mathrm{I}_{\mathrm{DS}}$ em função de $\mathrm{V}_{\mathrm{GF}}$ experimentais, com $\mathrm{V}_{\mathrm{DS}}=50 \mathrm{mV}$ and $1,5 \mathrm{~V}$, para transistores SOI convencionais e de canal gradual para diferentes comprimentos de canal.

\begin{tabular}{cccc}
\hline $\begin{array}{c}\text { Comprimento de } \\
\text { Canal }[\mu \mathrm{m}]\end{array}$ & Transistor & $\mathbf{n}\left(\mathbf{V}_{\mathbf{D S}}=\mathbf{5 0} \mathbf{~ m V}\right)$ & $\mathbf{n}\left(\mathbf{V}_{\mathbf{D S}}=\mathbf{1 , 5} \mathbf{V}\right)$ \\
\hline \multirow{2}{*}{2,00} & SOI Convencional & 1,083 & 1,087 \\
\cline { 2 - 4 } & GC SOI - LLD/L=0,35 & 1,098 & 1,099 \\
\cline { 2 - 4 } & GC SOI - LLD/L=0,50 & 1,100 & 1,087 \\
\cline { 2 - 4 } & GC SOI - LLD/L=0,62 & 1,098 & 1,099 \\
\hline \multirow{2}{*}{1,00} & SOI Convencional & 1,126 & 1,124 \\
\cline { 2 - 4 } & GC SOI - LLD/L=0,53 & 1,131 & 1,134 \\
\hline \multirow{2}{*}{0,75} & SOI Convencional & 1,350 & 1,338 \\
\cline { 2 - 4 } & GC SOI - L LD $/ L=0,25$ & 1,338 & 1,345 \\
\hline
\end{tabular}

Comparando os resultados de transistores GC SOI com comprimento total de canal de $2 \mu \mathrm{m}$ e comprimento efetivo semelhante ao comprimento de transistores convencionais ( $L_{\text {eff }}=1 \mu \mathrm{m}$ e $0,75 \mu \mathrm{m}$ ), observa-se que a estrutura de canal gradual permite a obtenção de seguidores de fonte com maior ganho, devido à menor condutância de dreno destes, sem degradação significativa, ao contrário do comportamento apresentado pelos transistores convencionais.

Quando transistores GC com comprimento efetivo de canal semelhante $\left(L_{\text {eff }} \cong\right.$ $0,56 \mu \mathrm{m})$ e diferentes comprimentos totais são comparados, observa-se a redução de $A_{V}$ com o comprimento de máscara em toda a excursão de $V_{\mathbb{I N}}$, devido ao 
aumento do fator de corpo, discutido anteriormente. Apesar desta redução, o ganho ainda permanece praticamente constante com a variação de $\mathrm{V}_{\mathrm{IN}}$.

Analisando a distorção harmônica total para os diferentes comprimentos de canal em inversão fraca, o mesmo comportamento observado na Figura 58(A) foi observado, ou seja, os níveis de distorção dos transistores GC e convencionais com mesmo comprimento total de canal, permanecem próximos.

A Figura 61 apresenta a distorção harmônica total em função de $V_{\mathbb{I N}}$, extraída a partir das curvas apresentadas na Figura 60(B) (inversão forte), considerando Va= $50 \mathrm{mV}$. A partir das curvas apresentadas, verifica-se que, sob o ponto de vista da linearidade, a utilização de transistores GC SOI apresenta vantagens em relação ao transistor convencional, tanto para o mesmo comprimento total de canal como para o mesmo comprimento efetivo. A melhora da linearidade para seguidores de fonte com o mesmo comprimento total de canal foi discutida na seção 4.4.1.2.

No caso de seguidores de fonte com o mesmo comprimento efetivo de canal (na Figura, $L_{\text {eff }}=1 \mu \mathrm{m}$ e 0,75 $\mu \mathrm{m}$ ), embora $\mathrm{g}_{\mathrm{m}}$ do transistor $\mathrm{GC}$ e convencional apresente valores semelhantes, $g_{D}$ ainda é menor nos transistores de canal gradual, justificando a menor distorção. Considerando o pior nível de THD extraído, foi observada uma melhora de pelo menos $10 \mathrm{~dB}$ na linearidade dos seguidores de fonte quando implementados com transistores de canal gradual.

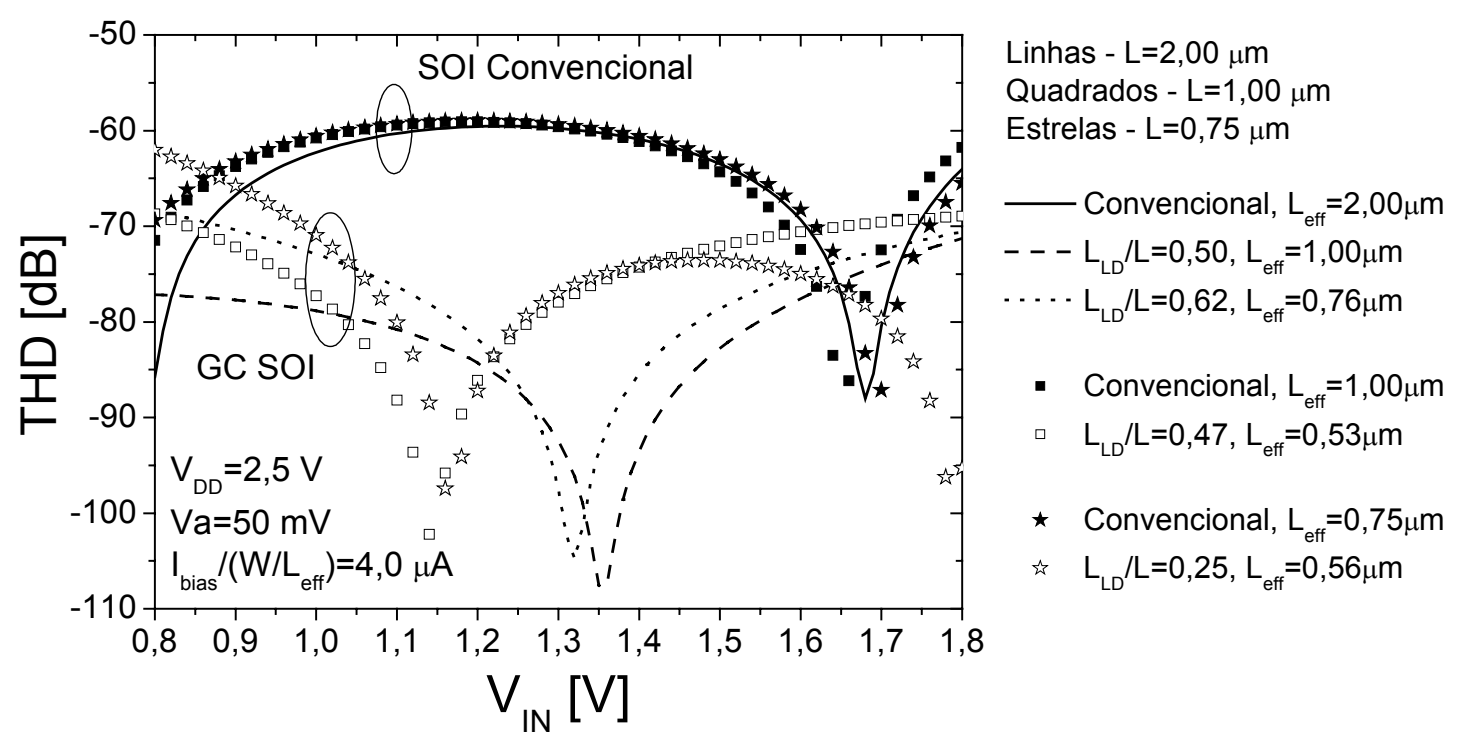

Figura 61 - Distorção harmônica total em função de $V_{\text {IN }}$ para seguidores de fonte operando em inversão forte, polarizados com $\mathrm{I}_{\text {bias }} /\left(\mathrm{W} / \mathrm{L}_{\text {eff }}\right)$ e $\mathrm{V}_{\mathrm{DD}}=2,5 \mathrm{~V}$, considerando $\mathrm{Va}=50 \mathrm{mV}$. 


\subsubsection{Simulações numéricas bidimensionais}

Foram também realizadas simulações numéricas de seguidores de fonte implementados com transistores GC e convencional com os mesmos parâmetros e polarizações utilizados anteriormente (seção 4.4.2) com diferentes comprimentos de canal ( $L=1 ; 2 ; 4$ e $10 \mu \mathrm{m})$.

A Figura 62 apresenta o ganho de tensão em função de $V_{\mathbb{I N}}$ obtido a partir da derivada numérica das curvas $V_{\text {OUt }}$ em função de $V_{\mathbb{I N}}$ para seguidores de fonte implementados com transistores $G C$ e convencionais com $L_{L D} / L=0,2$ para todos os comprimentos de canal simulados, em inversão fraca e forte. A partir destas curvas pode-se notar que, para transistores com comprimento de canal de $10 \mu \mathrm{m}$ até $2 \mu \mathrm{m}$, o ganho permanece inalterado se a estrutura de canal gradual for utilizada. No caso de dispositivos com comprimento de canal menor $(1 \mu \mathrm{m})$, uma redução do ganho é observada, devido ao aumento do fator de corpo. Resultados semelhantes foram obtidos para transistores $\mathrm{GC}$ com relações $\mathrm{LLD}_{\mathrm{LD}} / \mathrm{L}$ maiores. Avaliando $\mathrm{O}$ comportamento do ganho com a redução de $V_{\mathbb{I N}}$, observa-se que, como esperado, seguidores de fonte implementados com transistores uniformemente dopados sofrem maior degradação e esta aumenta com a redução do comprimento de canal e nível de inversão. Por outro lado, transistores de canal gradual apresentam pouca ou nenhuma degradação.

Os resultados do ganho apresentados na Figura 62 permitem observar que, a fim de implementar um seguidor de fonte com transistor uniformemente dopado, que apresente ganho semelhante àquele obtido com um transistor GC SOI com L=2 $\mu \mathrm{m}$, seria necessário aumentar o comprimento de canal em 5 vezes.

A Figura 63 apresenta a distorção harmônica total em função de $V_{\mathbb{I N}}$ extraída em inversão forte, com $\mathrm{Va}=50 \mathrm{mV}$, para os mesmos transistores apresentados na Figura 62. A partir das curvas obtidas, verifica-se que o uso de transistores de canal gradual como seguidor de fonte melhora sua linearidade em comparação com transistores de canal uniformemente dopado para todos os comprimentos de canal estudados. A melhora observada na linearidade é tanto maior quanto mais longo for o canal do transistor, e atinge mais de $20 \mathrm{~dB}$ no caso de transistores com $\mathrm{L}=10 \mu \mathrm{m}$. À medida que o comprimento de canal é reduzido, a melhora da linearidade diminui, 
sugerindo que a degradação de $g_{\mathrm{D}}$ é maior do que o aumento de $\mathrm{g}_{\mathrm{m}}$ causado pela redução do comprimento de canal.
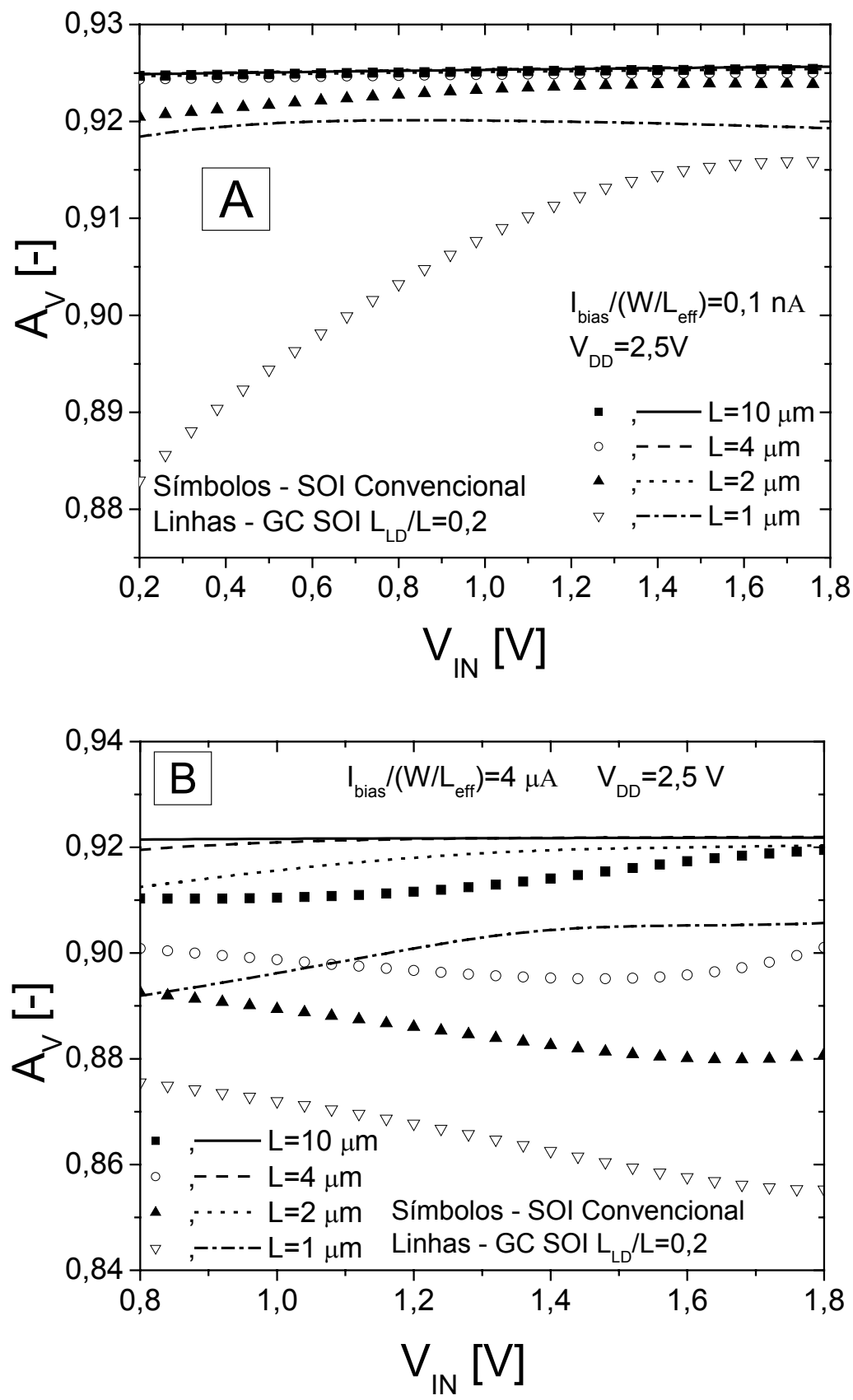

Figura 62 - Curvas $A_{V}$ em função de $V_{I N}$ simuladas para seguidores de fonte implementados com transistores convencionais e GC SOI com $L_{L D} / L=0,2$ e diferentes comprimentos de canal, em inversão fraca $(A)$ e forte $(B), c o m I_{\text {bias }} /\left(W / L_{\text {eff }}\right)=0,1 \mathrm{nA}$ e $4,0 \mu \mathrm{A}$, com $\mathrm{V}_{\mathrm{DD}}=2,5 \mathrm{~V}$.

Considerando o pior nível de distorção obtido para cada transistor, pode-se notar que a linearidade de seguidores de fonte implementados com transistores de 
canal gradual com $\mathrm{L}=2 \mu \mathrm{m}$ apresenta comportamento semelhante ao do transistor convencional com $L=10 \mu \mathrm{m}$. Assim, a simples substituição do transistor convencional por um de canal gradual, permite a implementação de seguidores de fonte com dimensões reduzidas, sem perda de ganho ou linearidade.

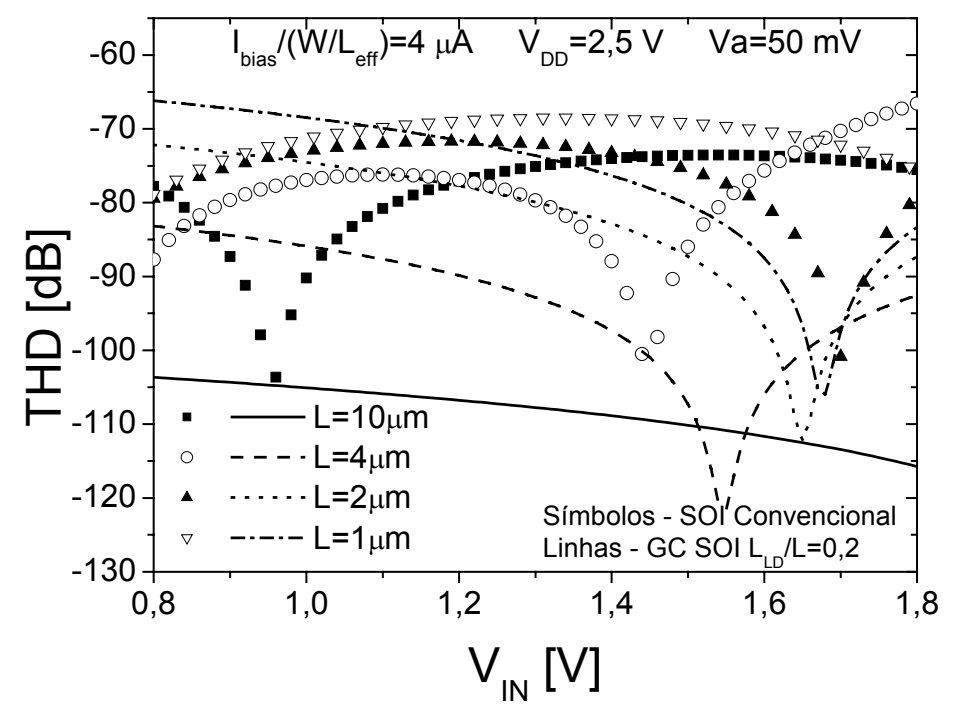

Figura 63 - THD em função de $\mathrm{V}_{\text {IN }}$ extraídas das curvas $\mathrm{V}_{\mathrm{OUT}}$ em função de $\mathrm{V}_{\text {IN }}$ simuladas em inversão forte, para transistores convencionais e de canal gradual com diferentes comprimentos de canal, considerando $\mathrm{Va}=50 \mathrm{mV}$.

\subsubsection{Operação em baixas temperaturas}

\subsubsection{Resultados experimentais}

Com o intuito de avaliar o comportamento de seguidores de fonte em baixas temperaturas, transistores de canal gradual e uniformemente dopado tiveram sua temperatura variada de $300 \mathrm{~K}$ a $90 \mathrm{~K}^{140}$, usando o Sistema K20 da MMR Technologies e as curvas experimentais foram obtidas utilizando ao Sistema de Caracterização de Semicondutores Keithley 4200. Foram utilizadas as mesmas correntes de dreno normalizadas aplicadas nos casos anteriormente estudados. Entretanto, neste caso, a tensão de polarização foi reduzida para $1,5 \mathrm{~V}$, a fim de 
minimizar a ocorrência do efeito de ionização por impacto, já agravada em baixas temperaturas.

A Figura 64 apresenta as curvas $V_{\text {OUT }}$ em função de $V_{\text {IN }}$ obtidas para transistores de $2 \mu \mathrm{m}$ de comprimento de canal, polarizados como seguidores de fonte, em inversão fraca e forte, com corrente de dreno normalizada de $0,1 \mathrm{nA}$ e $4 \mu \mathrm{A}$, medidas em $90 \mathrm{~K}$ e $300 \mathrm{~K}$. A partir das curvas apresentadas para seguidores de fonte operando em inversão fraca, pode-se notar que, para cada temperatura, um dado $V_{\mathbb{I N}}$ causa valores semelhantes de $\mathrm{V}_{\text {Out }}$ para todos os dispositivos, devido à semelhança entre as tensões de limiar e densidade de corrente para todos os transistores. Além disso, a redução da temperatura leva à diminuição da excursão de saída de todos os seguidores de fonte, causada pelo aumento da tensão de limiar.
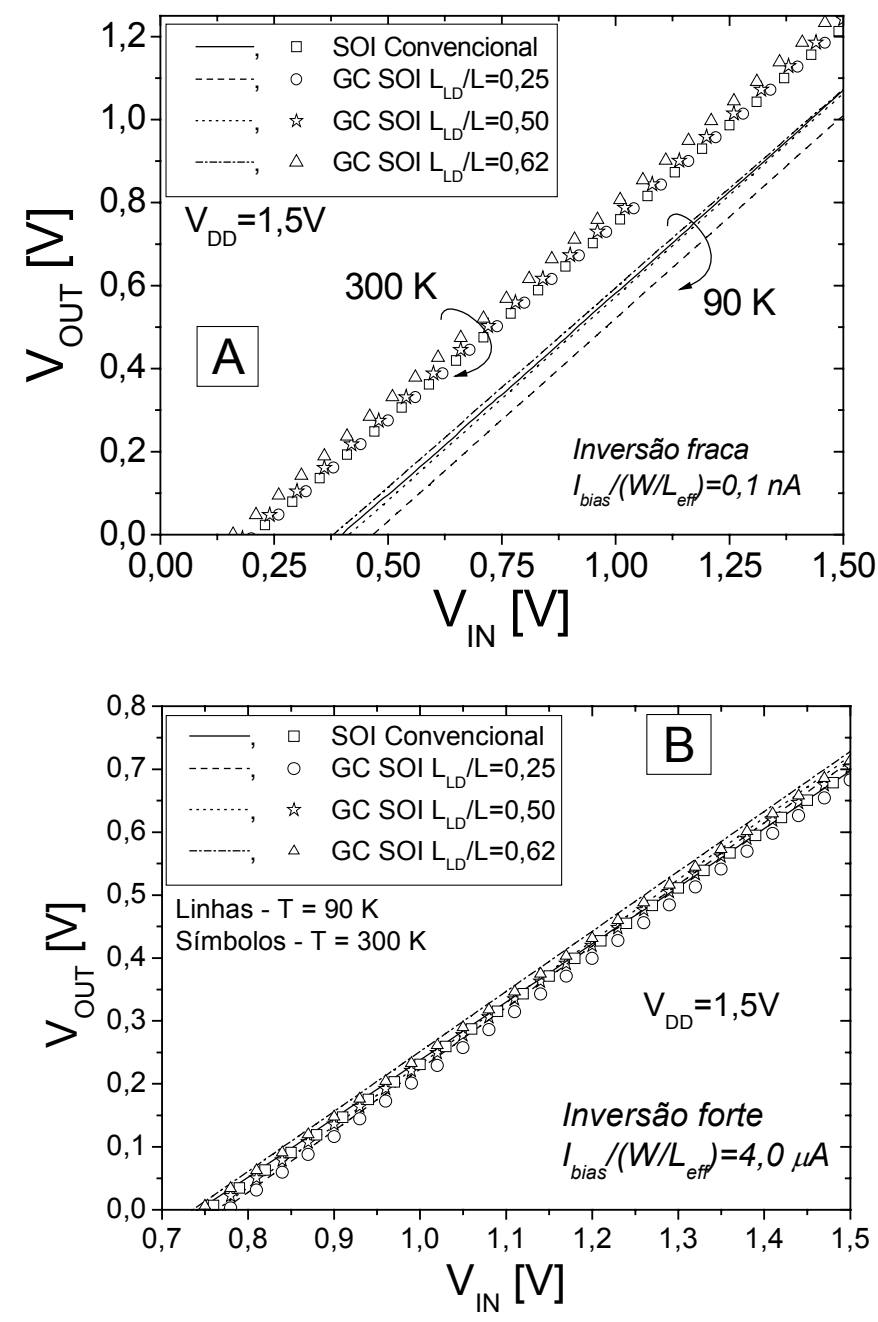

Figura 64 - Curvas $V_{\text {OUT }}$ em função de $V_{\text {IN }}$ de seguidores de fonte implementados com transistores convencional e GC SOI com $L=2 \mu \mathrm{m}$, medidas em inversão fraca (A) e forte (B), em $90 \mathrm{~K}$ e $300 \mathrm{~K}$. 
Em inversão forte, as curvas $V_{\text {OUT }}$ em função de $V_{\text {IN }}$ apresentam valores semelhante de $\mathrm{V}_{\text {OUT }}$ para um determinado $\mathrm{V}_{\mathrm{IN}}$ para diferentes temperaturas, indicando que os transistores estão polarizados próximo ao ponto de polarização invariante com a temperatura (Zero Temperature Coefficient - ZTC ${ }^{141}$ ).

A Figura 65 apresenta as curvas do ganho em função da tensão de entrada, obtidas a partir dos resultados apresentados na Figura 64. A partir das curvas apresentadas pode-se notar que, no intervalo de temperaturas estudado, em todos os níveis de inversão, transistores de canal gradual são capazes de promover maior ganho que o transistor convencional, quando operando como seguidor de fonte. No caso de seguidores de fonte implementados com transistores de canal gradual, o ganho aumenta de 0,94 para 0,98 quando a temperatura é reduzida de $300 \mathrm{~K}$ para $90 \mathrm{~K}$, tanto em inversão fraca como forte, e é praticamente insensível à variação de $\mathrm{V}_{\mathrm{IN}}$. Entretanto, para o seguidor de fonte implementado com transistor SOI convencional, o ganho apresenta uma variação significativa a $90 \mathrm{~K}$, causada pela redução da tensão de entrada, principalmente em inversão forte.
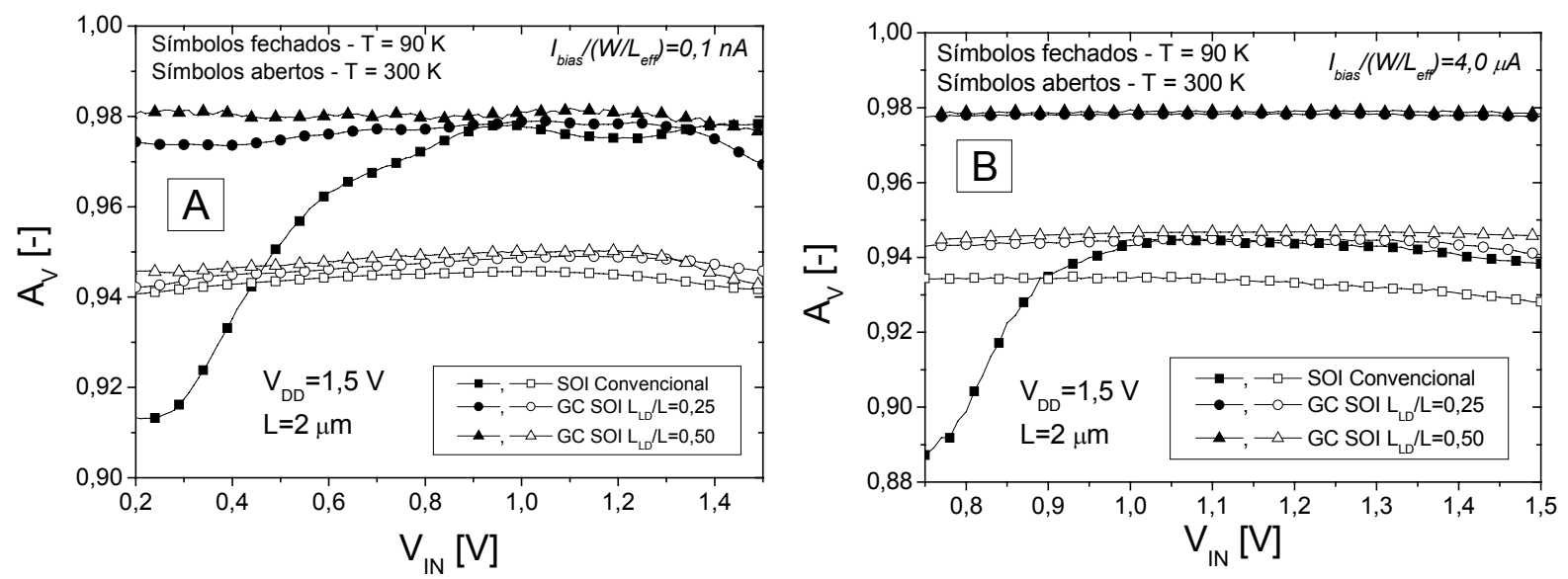

Figura 65 - Ganho em função da tensão de entrada, medidos para seguidores de fonte implementados com transistores convencional e GC SOI com $L=2 \mu \mathrm{m}$, medidas em inversão fraca (A) e forte (B), em $90 \mathrm{~K}$ e $300 \mathrm{~K}$.

\subsubsection{Simulações numéricas bidimensionais}

A fim de explicar a melhora do ganho promovido pela utilização de transistores GC SOI como seguidores de fonte operando em baixas temperaturas, 
foram realizadas simulações numéricas bidimensionais ${ }^{101}$, variando a temperatura entre $300 \mathrm{~K} \mathrm{e} 90 \mathrm{~K}$, e utilizando as mesmas condições de polarização utilizadas nas medidas experimentais (seção 4.4.4.1). Nestas simulações foram utilizados os parâmetros de modelo ajustados aos dados experimentais em temperatura ambiente, apresentados na seção 4.4 .2 e comprimento de canal de $2 \mu \mathrm{m}$.

$\mathrm{Na}$ Figura 66 são apresentadas as curvas do ganho de tensão em função de $\mathrm{V}_{\mathrm{IN}}$ para transistores $\mathrm{SOI}$ convencional e de canal gradual com $\mathrm{L}_{\mathrm{LD}} / \mathrm{L}=0,2$, configurados como seguidor de fonte operando em inversão forte, para diferentes temperaturas. Nestas curvas, pode-se verificar um aumento do ganho com a redução da temperatura, como nos resultados experimentais, embora seja menor que este, devido à não-otimização dos parâmetros do modelo de ionização por impacto dependentes da temperatura. Como visto nos resultados experimentais, seguidores de fonte implementados com transistores de canal gradual são capazes de proporcionas ganho praticamente constante durante toda a excursão de $V_{\mathbb{N}}$, em todas as temperaturas estudadas. Por outro lado, caso seja utilizado um transistor convencional, o ganho torna-se fortemente dependente da tensão de entrada, e a degradação observada é aumentada pela redução da temperatura, devido à maior ocorrência do efeito de ionização por impacto que, como apresentado anteriormente, é responsável pela redução do ganho.

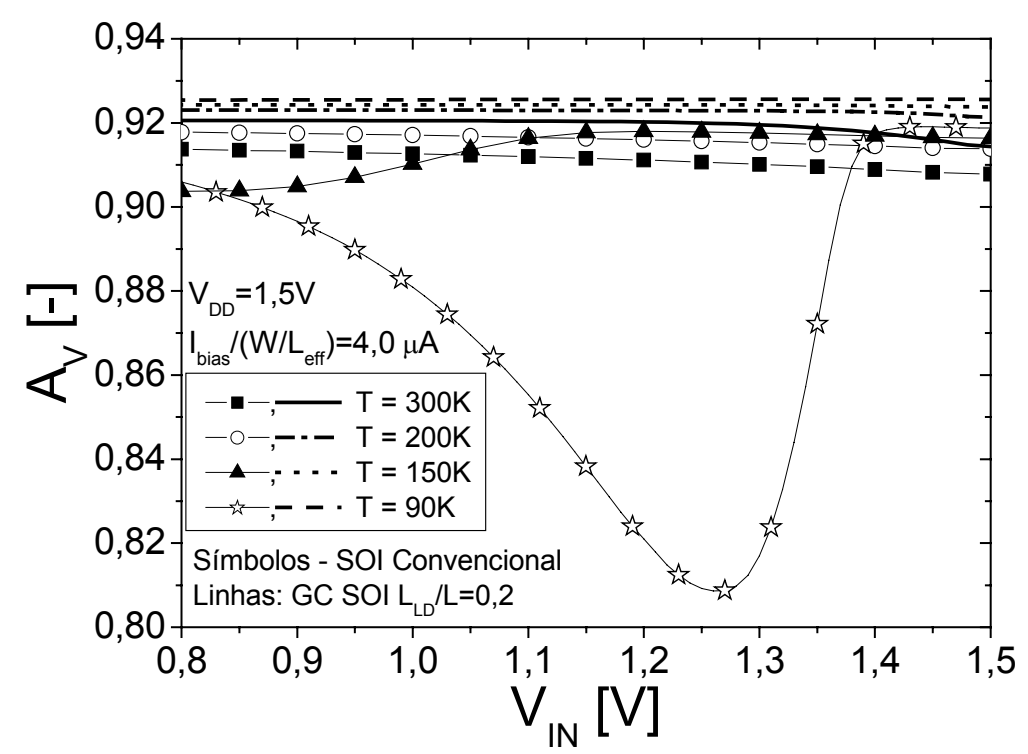

Figura 66 - Ganho de tensão para transistores convencional e GC SOI com LLD/L= 0,2 , configurados como amplificador dreno comum operando em inversão forte, para diferentes temperaturas. 
Com o objetivo de investigar o aumento do ganho com a redução da temperatura, da mesma forma realizada anteriormente, $g_{m}$ e $g_{D}$ foram extraídos a partir de curvas $I_{D S}$ em função de $V_{I N}\left(V_{G F}\right)$ e $I_{D S}$ em função de $V_{D S}$, com $V_{S}=0$, e $\mathrm{V}_{\mathrm{DS}}=\mathrm{V}_{\mathrm{IN}}=1 \mathrm{~V}$. Os resultados são apresentados na Tabela 10. São também apresentados os valores de $n . g_{m}+g_{D}$, denominado fator k. Para este cálculo, o fator de corpo $(n=1,09)$, extraído a partir da inclinação de sublimiar de curvas $I_{D S}$ em função de $V_{G F}$ com $V_{D S}=50 \mathrm{mV}$, não apresentou variação com a temperatura, uma vez que a presença de armadilhas de interface foram desconsideradas nos arquivos de simulação.

A variação dos parâmetros $g_{m}, g_{D} e k$ em relação à temperatura ambiente também é apresentada na Tabela 10. A partir destes resultados, pode-se notar que o aumento da transcondutância causado pela redução da temperatura é maior do que o aumento de k, tanto para o transistor convencional como para o de canal gradual. Os valores calculados para o ganho de tensão apresentados na tabela mostram um aumento do ganho de tensão com a redução da temperatura. Observando a equação do ganho (eq. (77)) pode-se notar que, uma vez que este é dado pela relação entre estes dois parâmetros $\left(g_{m} / k\right)$, para que $A_{V}$ aumente com a redução da temperatura, é necessário que o aumento de $g_{m}$ seja maior que o aumento de $k$. Como pode ser visto na tabela, a variação do termo k com a temperatura é menor no transistor de canal gradual do que no transistor convencional, justificando o maior aumento de $A_{V}$ observado experimentalmente nestes transistores.

A menor variação do termo $\mathrm{k}$ pode ser atribuída ao comportamento da condutância de dreno com a variação da temperatura. Os valores apresentados mostram que, para um transistor GC SOI com $V_{\mathbb{I N}}$ constante, gD diminui ligeiramente com a redução da temperatura. Tal redução deve-se ao aumento da tensão de limiar que, associado ao valor constante de $\mathrm{V}_{\mathrm{IN}}$, reduz a sobretensão de condução $\left(\mathrm{V}_{\mathrm{GT}}=\mathrm{V}_{\mathrm{IN}}-\mathrm{V}_{\mathrm{thf}}\right)$, promovendo a redução de $\mathrm{g}_{\mathrm{D}}{ }^{102}$. No caso do transistor convencional, ao contrário do comportamento apresentado por transistores de canal gradual, apresenta um degradação de $g_{D}$ em baixas temperaturas. Esta degradação indica que a ocorrência de ionização por impacto nesta estrutura é suficiente para compensar a redução da condutância de dreno promovida pela diminuição de $\mathrm{V}_{\mathrm{GT}}$. 
Tabela 10 - Valores simulados de $g_{m}, g_{D}$ e $k$ e sua variação com a temperatura, e ganho calculado, extraídos com $\mathrm{V}_{\mathbb{I N}_{\mathrm{N}}}=\mathrm{V}_{\mathrm{DS}}=1 \mathrm{~V}$ para seguidores de fonte implementados com transistores convencional e GC SOI com $L_{L D} / L=0,20$.

\begin{tabular}{|c|c|c|c|c|c|c|}
\hline & \multicolumn{3}{|c|}{ Convencional } & \multicolumn{3}{|c|}{ GC SOI $L_{L D} / L=0,20$} \\
\hline & $90 \mathrm{~K}$ & $200 \mathrm{~K}$ & $300 \mathrm{~K}$ & $90 \mathrm{~K}$ & $200 \mathrm{~K}$ & $300 \mathrm{~K}$ \\
\hline$g_{m}[\mu \mathrm{S}]$ & 42,20 & 27,10 & 17,20 & 47,30 & 32,00 & 20,40 \\
\hline$g_{D}[\mu S]$ & 0,69 & 0,61 & 0,51 & 0,10 & 0,22 & 0,35 \\
\hline $\mathrm{k}=\left(\mathrm{n} \cdot \mathrm{g}_{\mathrm{m}}+\mathrm{g}_{\mathrm{D}}\right)[\mu \mathrm{S}]$ & 46,60 & 30,00 & 19,30 & 51,60 & 35,10 & 22,70 \\
\hline$\left(g_{m, T}-g_{m, 300 K}\right) / g_{m, 300 K}$ & 1,45 & 0,58 & - & 1,32 & 0,57 & - \\
\hline$\left(g_{D, T}-g_{D, 300 K}\right) / g_{D, 300 K}$ & 0,34 & 0,20 & - & $-0,07$ & $-0,04$ & - \\
\hline$\left(\mathrm{k}_{\mathrm{T}}-\mathrm{k}_{300 \mathrm{~K}}\right) / \mathrm{k}_{300 \mathrm{~K}}$ & 1,41 & 0,55 & - & 1,27 & 0,55 & - \\
\hline$A_{V}=g_{m} / k$ & 0,91 & 0,90 & 0,89 & 0,92 & 0,91 & 0,90 \\
\hline
\end{tabular}

SOUZA, M. de; FLANDRE, D.; PAVANELLO, M. A.; Advantages of Graded-Channel SOI nMOSFETs for Application as Source-Follower Analog Buffer. Artigo aceito para publicação no periódico Solid-State Electronics, 2008.

SOUZA, M. de; FLANDRE, D.; PAVANELLO, M. A.; Improved Source-Follower Buffer Implementation by Using Graded-Channel SOI nMOSFETs. EuroSOI 2008 Conference Proceedings, v. 1, p. 35-36, 2008.

SOUZA, M. de; FLANDRE, D.; PAVANELLO, M. A. Channel Length Influence on the Performance of Source-Follower Buffers Implemented with Graded-Channel SOI nMOSFETs. Microelectronics Technology and Devices - SBMicro2008. v.14, p.263272, 2008.

SOUZA, M. de, FLANDRE, D., PAVANELLO, M. A. Low Temperature and Channel Engineering Influence on the Behavior of Analog Source-Follower Buffers Proceedings of the Eighth International Workshop on Low Temperature Electronics. v.1, p.32-33, 2008. 


\subsection{ESPELHOS DE CORRENTE IMPLEMENTADOS COM TRANSISTORES GC SOI OPERANDO EM BAIXAS TEMPERATURAS}

Espelhos de corrente são blocos bastante utilizados em circuitos analógicos, seja para a polarização de diversos ramos de um circuito, como para operar como carga ativa. A operação básica de um espelho de corrente consiste em fornecer uma corrente de saída, $\mathrm{l}_{\mathrm{OUT}}$, proporcional à corrente de entrada, $\mathrm{I}_{\mathbb{N}}$, independente da tensão aplicada ao dreno do transistor de saída, $V_{D, O U T}{ }^{121}$. Desta forma, para circuitos implementados com transistores idênticos, a precisão de espelhamento, dada pela relação $\mathrm{l}_{\mathrm{OUt}} / \mathrm{I}_{\mathrm{IN}}$ deve ser o mais próxima possível da unidade, em todos os regimes de inversão.

A utilização de transistores GC SOI em espelhos de corrente proporciona vantagens em relação aos transistores SOI convencionais, dentre as quais pode-se citar a maior precisão de espelhamento e aumento da excursão de saída e da resistência de saída. Essas vantagens já foram apresentadas, em temperatura ambiente, para espelhos de corrente nas arquiteturas fonte comum $^{142}$, Wilson e Cascode ${ }^{94}$, projetados e apresentados na seção 4.1.

Nesta seção apresentamos os primeiros resultados experimentais da operação em baixa temperatura de espelhos de corrente nas arquiteturas Wilson e Cascode (Figura 67), implementados com transistores de canal gradual..

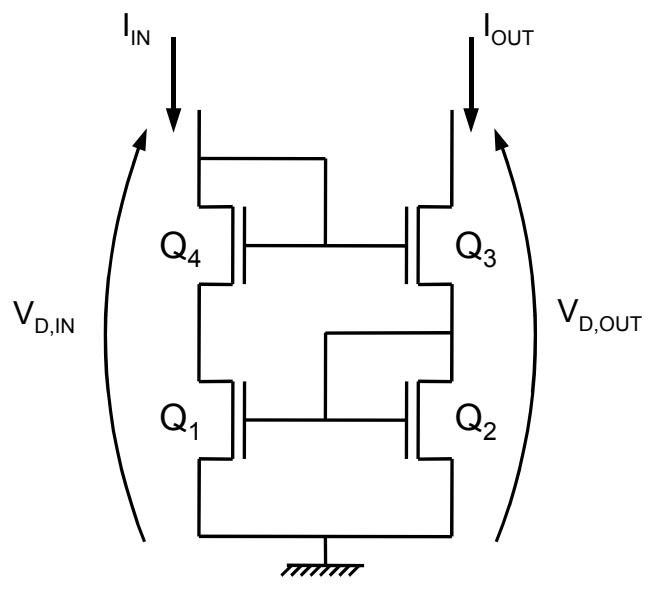

(A)

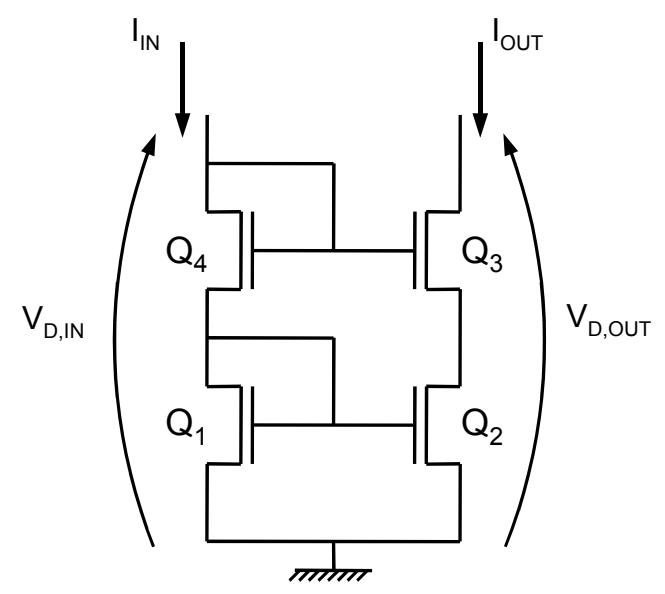

(B)

Figura 67 - Representação esquemática de espelhos de corrente nas arquiteturas Wilson (A) e Cascode (B). 
A precisão de espelhamento é um parâmetro dependente das características de casamento dos transistores e da polarização aplicada ${ }^{143}$. O descasamento intrínseco ocorre devido a diferenças na tensão de limiar e nas dimensões dos transistores que compõem o espelho de corrente ${ }^{121}$. Com o objetivo de avaliar este parâmetro, os espelhos de corrente foram submetidos a testes experimentais aplicando a mesma tensão à sua entrada e saída ( $\mathrm{V}_{\mathbb{I N}}$ e $\mathrm{V}_{\text {OUT, }}$, respectivamente). As curvas $\mathrm{l}_{\mathrm{OUT}} / \mathrm{I}_{\mathbb{N}}$ em função da corrente de entrada normalizada, obtidas, em diversas temperaturas, são apresentadas na Figura 68, para espelhos de corrente implementados com transistores convencional e GC SOI, sendo $L=2 \mu \mathrm{m}$ para as duas arquiteturas.
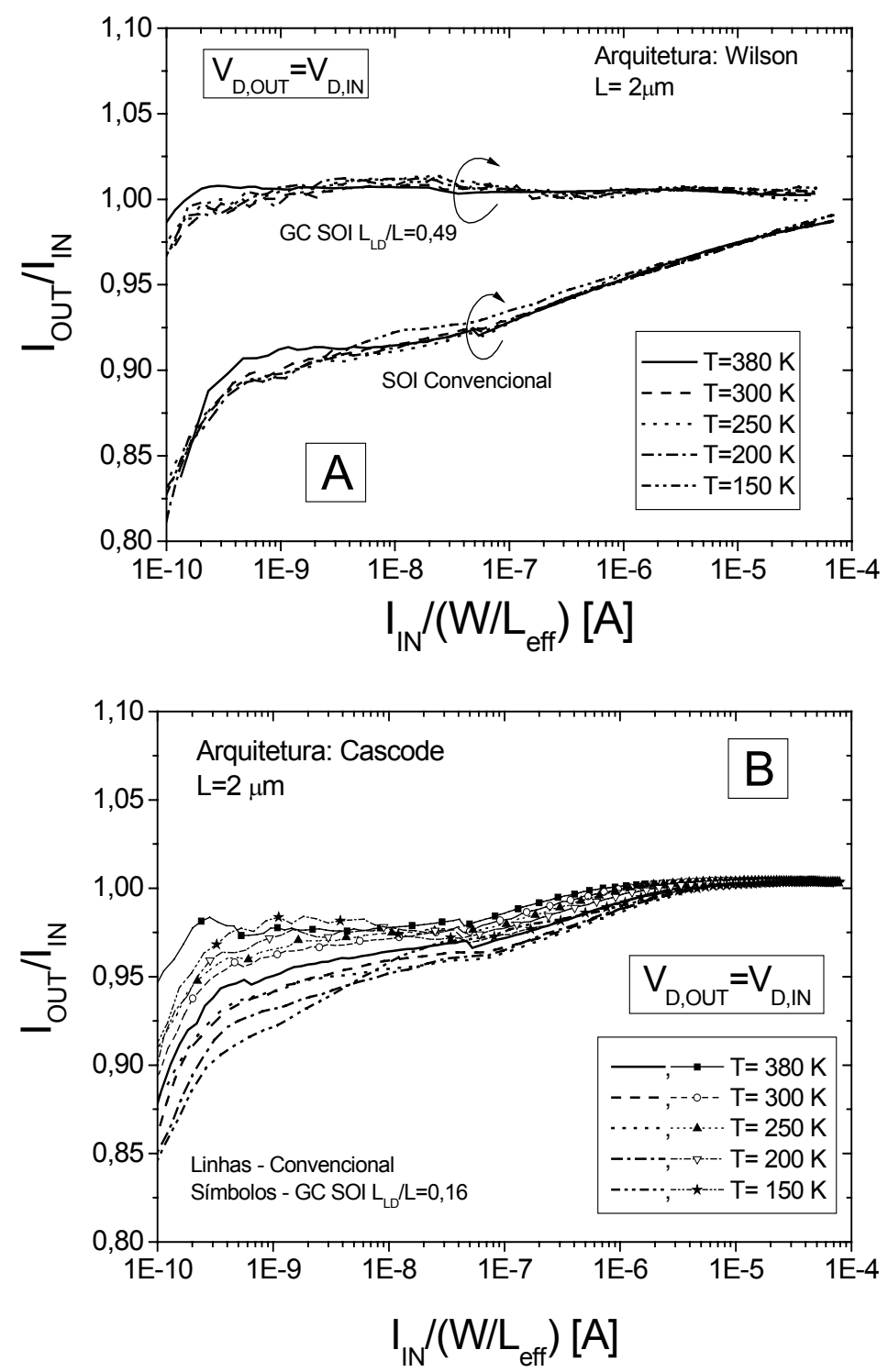

Figura 68 - Precisão de espelhamento em função da corrente de entrada normalizada, obtidas experimentalmente, para espelhos de corrente nas arquiteturas Wilson (A) e Cascode (B), com a temperatura variando de $380 \mathrm{~K}$ a $150 \mathrm{~K}$. 
A partir das curvas apresentadas pode-se notar que, os espelhos de corrente que utilizam transistores GC apresentam melhor precisão de espelhamento do que aqueles implementados com transistores convencionais, para todas as temperaturas. Além disso, ao variar a temperatura, a relação $\mathrm{l}_{\mathrm{OUT}} / \mathrm{I}_{\mathrm{IN}}$ permanece praticamente inalterada quando os espelhos de corrente operam em inversão forte. À medida que se aproximam da inversão fraca, os espelhos de corrente passam a apresentar uma variação em sua precisão causada pela variação da temperatura. Este comportamento está associado ao descasamento da tensão de limiar ${ }^{121}$, que é dominante neste regime de inversão e é piorado em baixas temperaturas, conforme apresentado na seção 4.3.4.

A Figura 69 apresenta curvas de $\mathrm{l}_{\mathrm{OUT}}$ em função de $\mathrm{V}_{\mathrm{D}, \mathrm{OUT}}$, medidas em $\mathrm{T}=150 \mathrm{~K}$ e $300 \mathrm{~K}$, com $\mathrm{I}_{\mathbb{N}}=1 \mu \mathrm{A}$, para espelhos de corrente nas duas arquiteturas estudadas, implementados com transistores SOI convencional e de canal gradual. A partir destas curvas pode-se notar que, para uma dada temperatura e com mesmo $I_{\mathbb{N}}$, a utilização de transistores GC SOI promove a redução da tensão de saturação dos espelhos de corrente $\left(\mathrm{V}_{\mathrm{SAT}, \mathrm{CM}}\right)$ e aumento de sua tensão de ruptura $\left(\mathrm{BV}_{\mathrm{CM}}\right)$. Por outro lado, a redução da temperatura, causa o efeito oposto, ou seja, o aumento da tensão de saturação e redução da tensão de ruptura, tanto para espelhos de corrente implementados com transistores uniformemente dopados como para os de canal gradual. Este comportamento pode ser claramente visto na Figura 70, que apresenta a tensão de saturação e de ruptura dos espelhos de corrente caracterizados em função da temperatura. Estes valores foram extraídos utilizando a técnica proposta na ref. [ 144 ]. Nesta técnica, são utilizadas curvas $\left[\mathrm{d}\left(1 / \mathrm{g}_{\mathrm{D}}\right) / \mathrm{dV}_{\mathrm{D}, \mathrm{OUT}} \times \mathrm{g}_{\mathrm{D}}\right]$ em função de $\mathrm{V}_{\mathrm{D}, \mathrm{OUT}}$, obtidas com $\mathrm{I}_{\mathrm{IN}}$ constante (neste caso, $\left.I_{\mathbb{N}}=1 \mu \mathrm{A}\right)$. Esta curva apresenta um pico positivo, que representa a tensão de saturação, e um pico negativo, através do qual é definida a tensão de ruptura. 

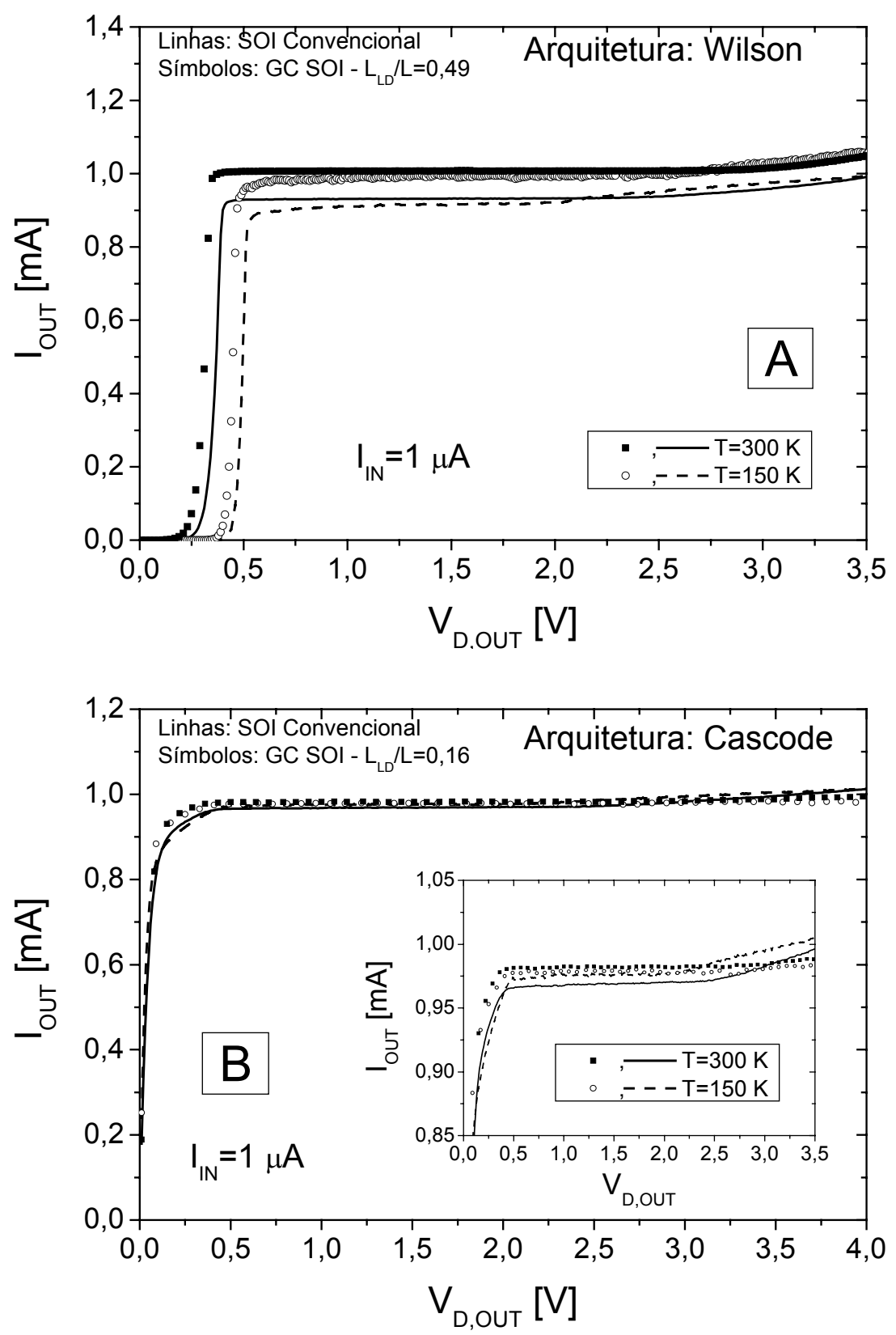

Figura 69 - Curvas lout em função de $V_{D, O U T}$ medidas para espelhos de corrente Wilson (A) e Cascode (B), com transistores SOI convencionais e de canal gradual, em $\mathrm{T}=150 \mathrm{~K}$ e $300 \mathrm{~K}$. 

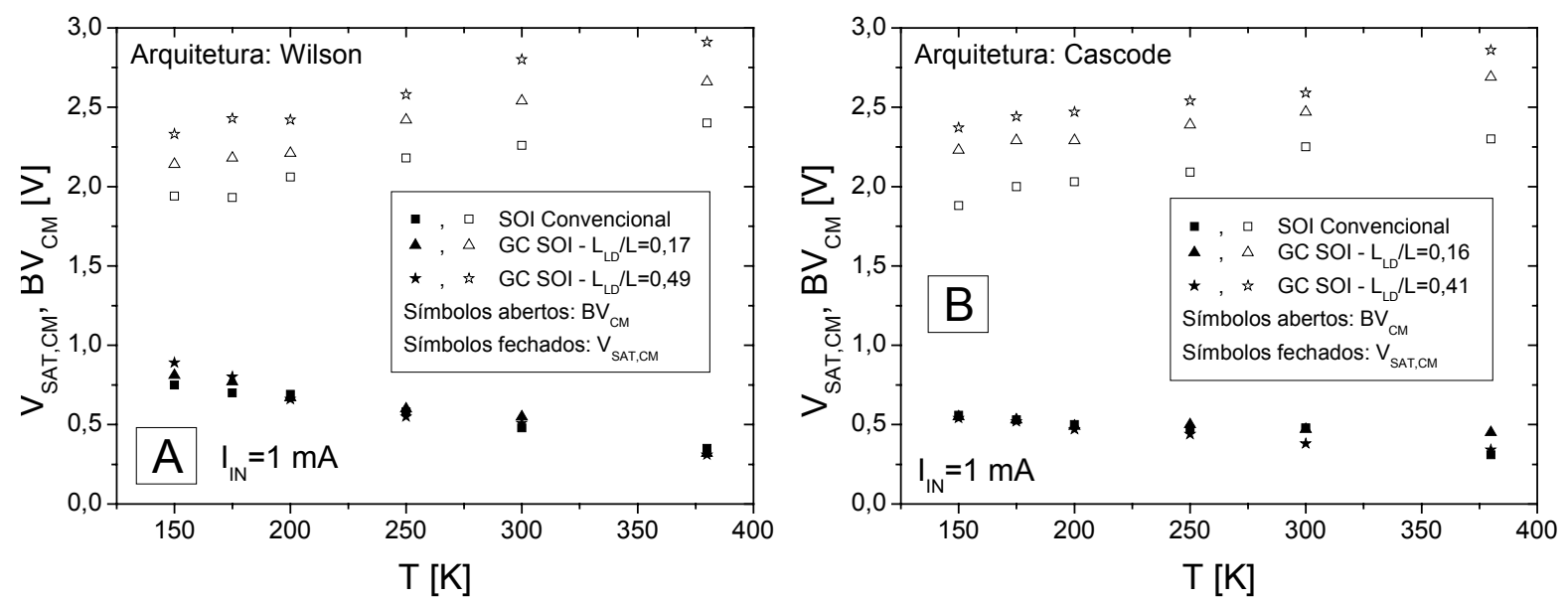

Figura 70 - Tensão de saturação e de ruptura em função da temperatura, extraídas a partir das curvas $I_{\text {OUt }}$ em função de $V_{D, O U T}$, com $I_{I_{N}}=1 \mu A$.

A excursão de saída, $V_{O S}$, dos espelhos de corrente caracterizados foi obtida a partir da diferença entre a tensão de saturação e de ruptura, e é apresentada, em função da temperatura, na Figura 71. Estas curvas mostram que, para as duas arquiteturas, a excursão de saída é maior nos espelhos de corrente que utilizam transistores de canal gradual do que aqueles com transistores uniformemente dopados, em todo o intervalo de temperaturas estudado. Como mencionado anteriormente, este aumento de $V_{O S}$ deve-se ao aumento da tensão de ruptura proporcionado pela presença da região fracamente dopada próxima ao dreno dos transistores GC SOI e redução da tensão de saturação. Como esperado, ao se reduzir a temperatura, verifica-se a diminuição de $V_{O S}$ para todos os espelhos de corrente, devido ao aumento da tensão de saturação e a redução da tensão de ruptura, mostrados na Figura 70. 

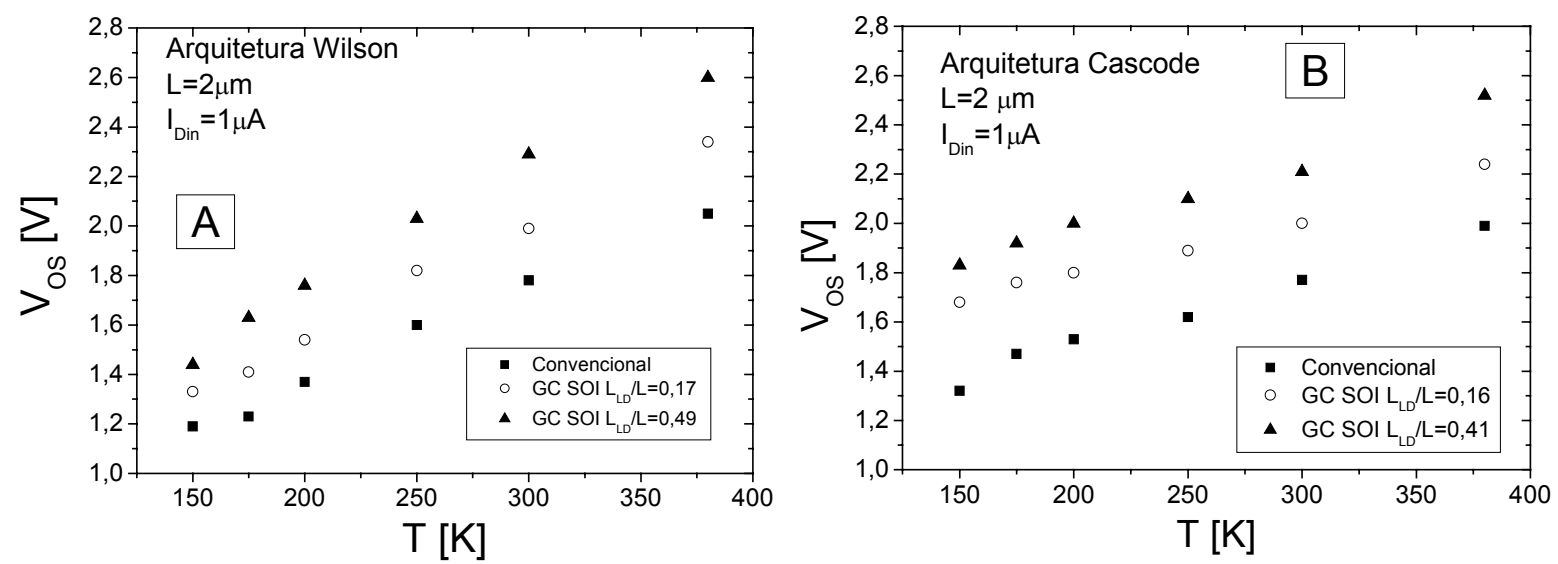

Figura 71 - Excursão de saída em função da temperatura extraída de espelhos de corrente implementados com transistores GC SOI e convencionais, nas arquiteturas Wilson (A) e Cascode (B).

Uma das vantagens da utilização de transistores de canal gradual na implementação de espelhos de corrente está associada ao aumento da resistência de saída $\left(R_{\mathrm{OUT}}\right)^{94}$.

De acordo com a ref. [78], a resistência de saída atingida por espelhos de corrente nas arquiteturas Wilson e Cascode é dada por

$$
\mathrm{R}_{\mathrm{OUT}} \approx \mathrm{g}_{\mathrm{m} 3} \cdot \mathrm{r}_{\mathrm{d} 3} \cdot \mathrm{r}_{\mathrm{d} 2}
$$

onde $g_{\mathrm{m} 3}$ é a transcondutância do transistor $\mathrm{Q} 3$, indicado na Figura 67 , e $r_{\mathrm{d} 2}$ e $r_{\mathrm{d} 3}$ são as resistências de saída dos transistores Q2 e Q3, respectivamente, dadas pelo inverso da condutância de dreno.

A partir da regressão linear das curvas lout em função de $V_{D, O U T}$ com $\mathrm{I}_{\mathrm{IN}}=1 \mu \mathrm{A}$, no intervalo $\mathrm{V}_{\mathrm{SAT}, \mathrm{CM}} \leq \mathrm{V}_{\mathrm{D}, \mathrm{OUT}} \leq \mathrm{BV}_{\mathrm{CM}}$, foram extraídos os valores de $\mathrm{R}_{\mathrm{OUT}}$ para os espelhos de corrente Wilson. A Figura 72 apresenta os resultados obtidos em função da temperatura. Além do aumento, superior a 4 vezes, em temperatura ambiente ${ }^{94}$, proporcionado pela utilização da estrutura de canal gradual, verifica-se a redução de Rout com a diminuição da temperatura em todos os espelhos de corrente, devido ao aumento da condutância de saída observada em baixas temperaturas, reduzindo $r_{\mathrm{d} 2}$ e $r_{\mathrm{d} 3}$. Entretanto, para todo o intervalo de temperaturas estudado, $R_{\text {OUt }}$ permanece maior nos espelhos de corrente com transistores GC SOI. 

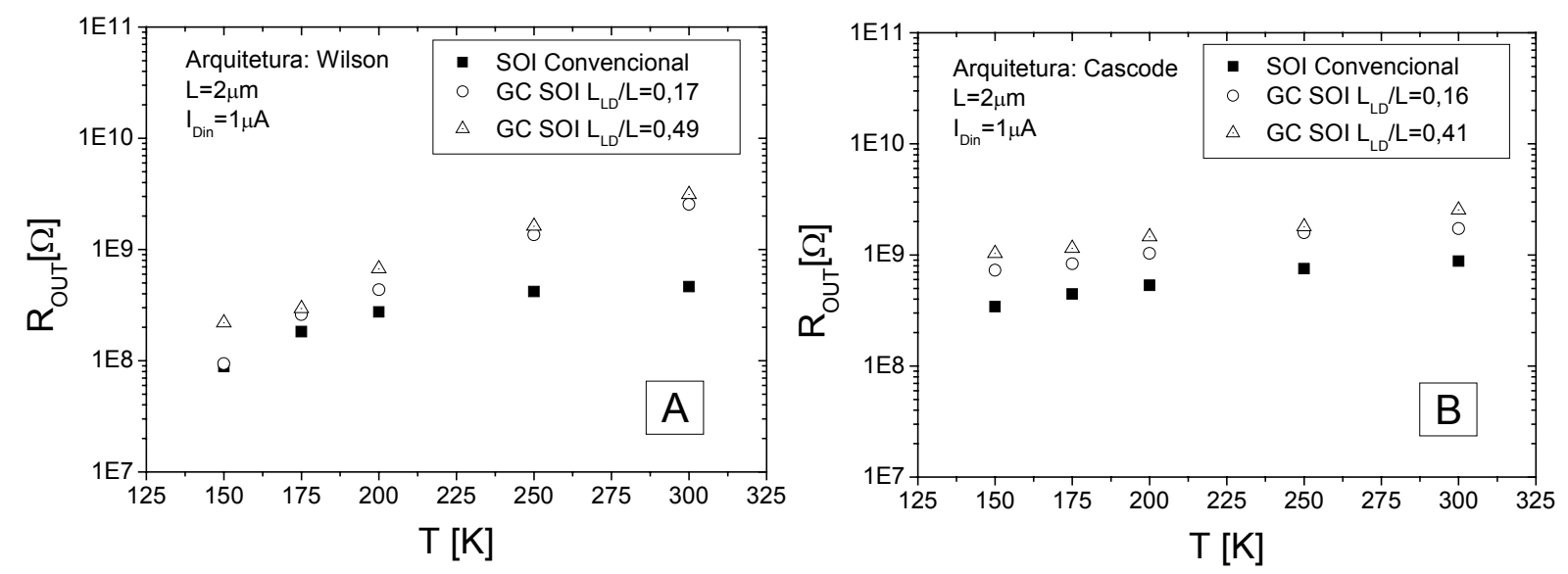

Figura 72 - Resistência de saída em função da temperatura para espelhos de corrente Wilson (A) e Cascode (B) implementados com transistores convencionais e de canal gradual. 


\section{TRANSISTORES SOI COM CANAL TENSIONADO}

Como mencionado na seção 2.1.3, o uso de tensão mecânica em tecnologias ultra-submicrométricas é de grande interesse para o contínuo escalamento das dimensões de dispositivos MOS, permitindo melhorar o desempenho dos dispositivos, através do aumento da mobilidade dos portadores.

Tendo em vista as melhorias de desempenho proporcionadas pela redução da temperatura, nesta seção será apresentado um estudo da operação de transistores SOI com tensão uniaxial agindo sobre o canal, operando entre $100 \mathrm{~K} \mathrm{e}$ $380 \mathrm{~K}$. Inicialmente será abordado o funcionamento de transistores com tensão uniaxial em função da temperatura para diversos comprimentos de canal. Em seguida, serão estabelecidas algumas comparações entre transistores com tensão uniaxial e biaxial com comprimento de canal de $160 \mathrm{~nm}^{145,146,147}$.

\subsection{CARACTERÍSTICAS DOS DISPOSITIVOS CARACTERIZADOS}

Transistores SOI totalmente depletados foram fabricados no Interuniversity Microelectronics Center - IMEC, Leuven, Bélgica, usando a tecnologia planar de porta única de $65 \mathrm{~nm}^{44}$. A porta dos dispositivos é formada por uma camada de $100 \mathrm{~nm}$ de silício policristalino e o isolante de porta utilizado é o oxinitreto de silício com $1,5 \mathrm{~nm}$ de espessura. A espessura final das camadas de silício e de óxido enterrado é de $15 \mathrm{~nm}$ e $150 \mathrm{~nm}$, respectivamente. Fonte e dreno elevados em $25 \mathrm{~nm}$, além de silicetação de fonte, dreno e porta com Níquel são utilizadas para reduzir a resistência série. Para este estudo, foram utilizados dois tipos de transistores SOI nMOSFETs com canal tensionado. No primeiro deles, a tensão uniaxial ao longo do canal (stress intrínseco de $800 \mathrm{MPa}$ ) é induzida através da deposição de uma camada de $100 \mathrm{~nm}$ de nitreto de silício (sCESL) em uma etapa adicional de processo. O segundo tipo, com tensão biaxial, é obtido através da utilização de lâminas SOI tensionadas (sSOI).

Transistores com comprimento efetivo de canal de $160 \mathrm{~nm}, 410 \mathrm{~nm}, 910 \mathrm{~nm}$ e 
$10 \mu \mathrm{m}$ e largura de canal de $1 \mu \mathrm{m}$ tiveram sua temperatura controlada através do equipamento Variable Temperature Micro Probe System, modelo K20 da MMR Technologies e as curvas de corrente foram extraídas utilizando o analisador de parâmetros de semicondutores Keithley 4200 e tempo de integração médio.

Para tecnologias modernas, devido ao uso de isolantes de porta ultra-finos, a corrente de porta $\left(I_{G}\right)$ não pode ser desprezada. Desta forma, a existência da corrente de porta foi compensada usando o método proposto na ref. [148].

De acordo com este método,

$$
I_{D}=I_{D 0}-I_{G D}
$$

onde $I_{D}$, e $I_{D 0}$ são a corrente de dreno e a corrente de dreno intrínseca, na ausência de corrente de porta. $O$ termo $I_{G D}$ refere-se à parcela da corrente de porta $\left(I_{G}\right)$ proveniente do dreno. Para baixas polarizações de dreno ${ }^{148}$,

$$
\frac{\mathrm{I}_{\mathrm{GD}}}{\mathrm{I}_{\mathrm{G}}} \cong 0,5
$$

Desta forma, utilizando as equações (80) e (81), a corrente de dreno intrínseca pode ser obtida por:

$$
I_{D 0}=I_{D}+0,5 \cdot I_{G}
$$

Para valores de tensão de dreno maiores, a relação expressa pela eq. (81) sofre alteração, como apresentado na ref. [148].

A Figura 73 apresenta as curvas da corrente de dreno, de porta e a corrente de dreno intrínseca, ambas em função de $V_{G F}$, calculada a partir da eq. (82), para um transistor SOI nMOSFET com L=10 $\mu \mathrm{m}$ e $\mathrm{W}=1 \mu \mathrm{m}$, polarizado com $\mathrm{V}_{\mathrm{DS}}=50 \mathrm{mV}$. 


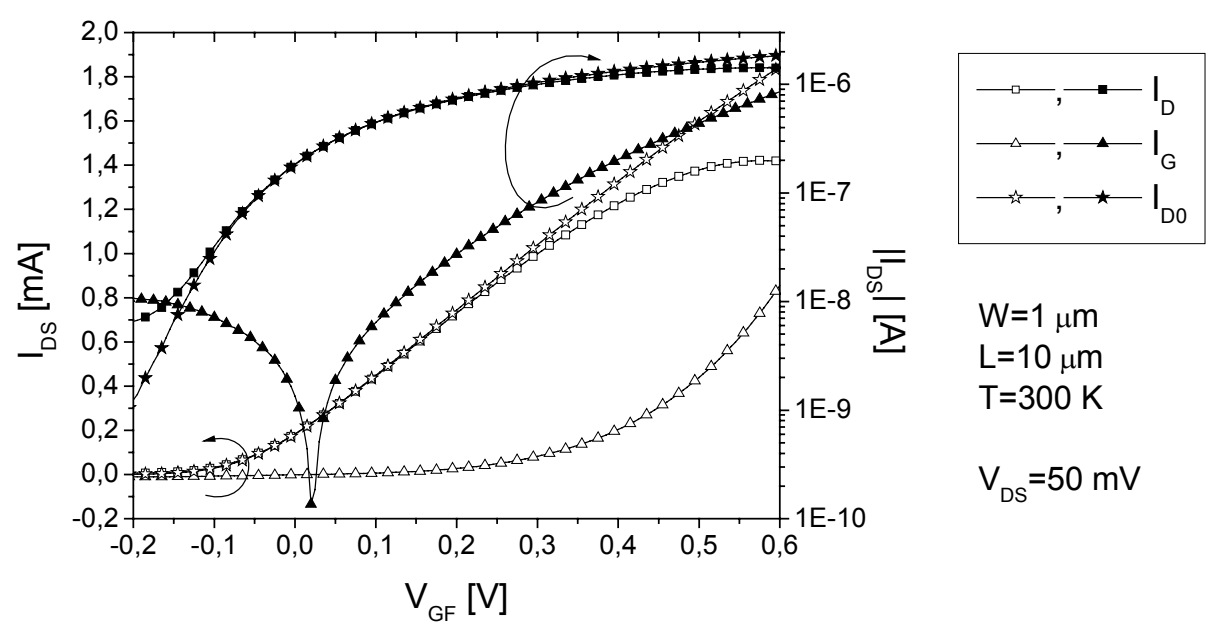

Figura 73 - Corrente de dreno, porta e corrente de dreno intrínseca na ausência da corrente de porta, todas em função da tensão de porta, para um transistor SOI nMOSFET convencional, com $\mathrm{L}=10 \mu \mathrm{m}$ e $\mathrm{W}=1 \mu \mathrm{m}$, polarizado com $\mathrm{V}_{\mathrm{DS}}=50 \mathrm{mV}$.

Ao longo desta seção, trataremos a corrente de dreno intrínseca apenas como corrente de dreno, IDS.

\subsection{OPERAÇÃO DE TRANSISTORES COM TENSÃO UNIAXIAL EM BAIXAS TEMPERATURAS}

\subsubsection{Ganho de transcondutância e inclinação de sublimiar}

A Figura 74(A) apresenta as curvas da corrente de dreno em função da sobretensão de condução, obtidas com $V_{D S}=50 \mathrm{mV}$, em $T=150 \mathrm{~K}$ e $300 \mathrm{~K}$, para transistores com e sem SCESL, e comprimento de canal de $160 \mathrm{~nm}$. A partir da derivada numérica das curvas $I_{D S}$ em função de $V_{G T}$, foram obtidas as curvas da transcondutância, apresentadas na mesma figura. A partir destes resultados, podese notar o aumento do nível de corrente e da transcondutância propiciados tanto pela presença do SCESL como pela redução da temperatura. 

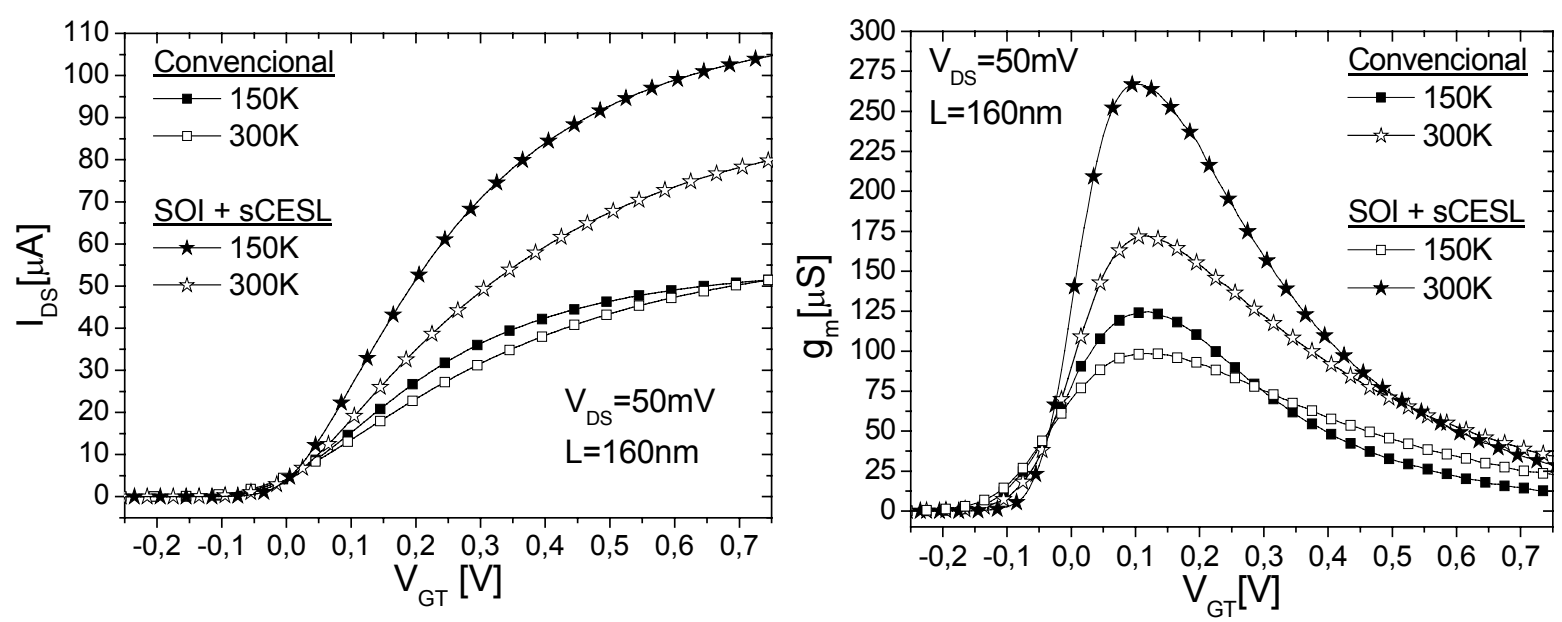

Figura 74 - Corrente de dreno $(A)$ e transcondutância $(B)$ em função da sobretensão de condução obtida com $V_{D S}=50 \mathrm{mV}$ para dispositivos com $\mathrm{L}=160 \mathrm{~nm}$ com e sem sCESL, em $150 \mathrm{~K}$ e $300 \mathrm{~K}$.

A fim de avaliar o ganho de mobilidade proporcionado pelo uso da tensão uniaxial causada pela presença do sCESL, a transcondutância máxima $\left(g_{m, m a x}\right)$ em região linear, com baixo campo elétrico, foi utilizada. A partir das curvas da corrente de dreno em função da tensão de porta, a transcondutância máxima foi extraída para todos os comprimentos de canal estudados. Os resultados de $\mathrm{g}_{\mathrm{m} \text {,max }}$ para todos os comprimentos de canal estudados, em função da temperatura, são apresentados na Figura 75. Nota-se que a transcondutância máxima (e a mobilidade) aumenta com a redução da temperatura para todos os dispositivos, como previsto na seção 2.3.4. Como esperado, o uso da tensão uniaxial proporcionada pelo sCESL é mais efetiva para aumentar a transcondutância para dispositivos de canal curto, em todas as temperaturas, como pode ser visto na Figura 76, que apresenta $g_{m, \max }$ em função do comprimento de canal para $\mathrm{T}=100 \mathrm{~K}, 200 \mathrm{~K}$ e $300 \mathrm{~K}$. 


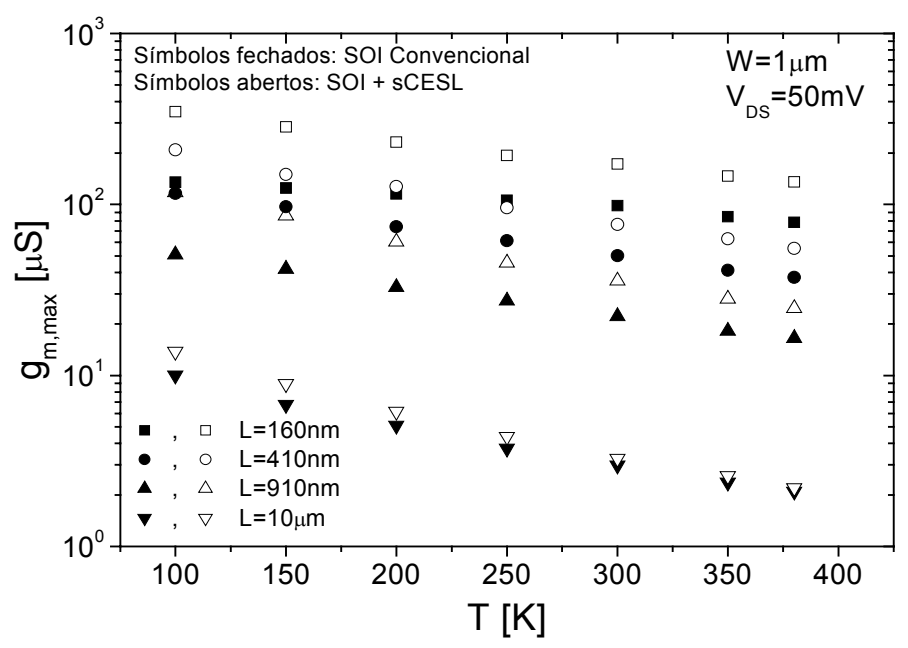

Figura 75 - Transcondutância máxima em função da temperatura para transistores com e sem sCESL com diferentes comprimentos de canal

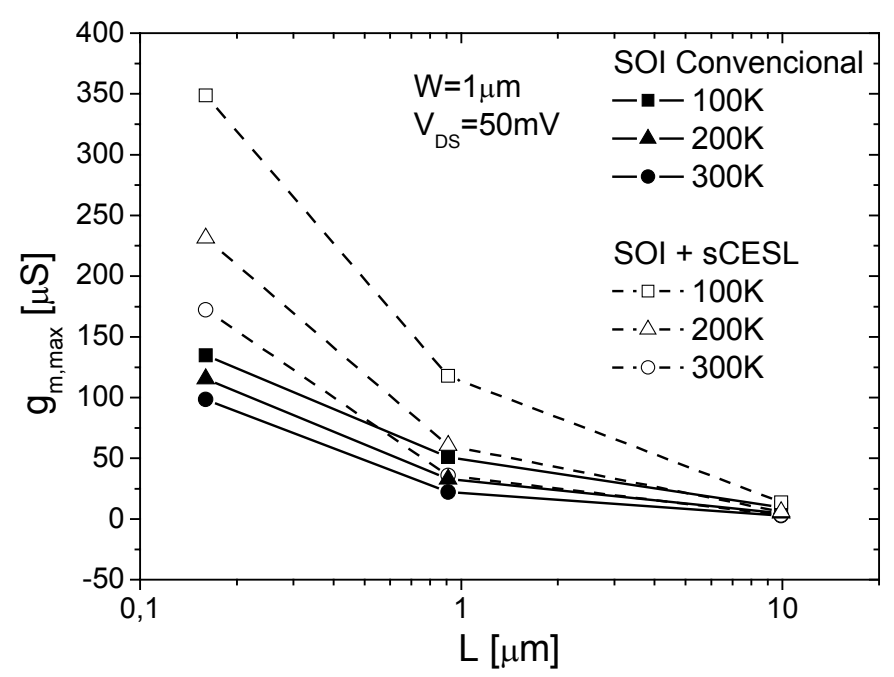

Figura 76 - Transcondutância máxima, obtida em região linear, em função do comprimento de canal com $\mathrm{T}=100 \mathrm{~K}, 200 \mathrm{~K}$ e $300 \mathrm{~K}$.

As curvas apresentadas na Figura 76 sugerem que o ganho de mobilidade (representada pelo aumento de $\mathrm{g}_{\mathrm{m}, \max }$ ), tende a aumentar com a diminuição da temperatura. Com o objetivo de confirmar esta melhora obtida pela tensão uniaxial, a Figura 77 apresenta o aumento relativo de $\mathrm{g}_{\mathrm{m}}$ proporcionado pela presença do SCESL em relação ao SOI convencional, em função da temperatura, para dispositivos com L=160 nm e $910 \mathrm{~nm}$. O ganho de mobilidade observado para o dispositivo com $L=160 \mathrm{~nm}$ é cerca de $83 \%$ maior em $100 \mathrm{~K}$ do que em $300 \mathrm{~K}$ e cerca de $70 \%$ maior para o dispositivo com $\mathrm{L}=910 \mathrm{~nm}$ nas mesmas temperaturas. 


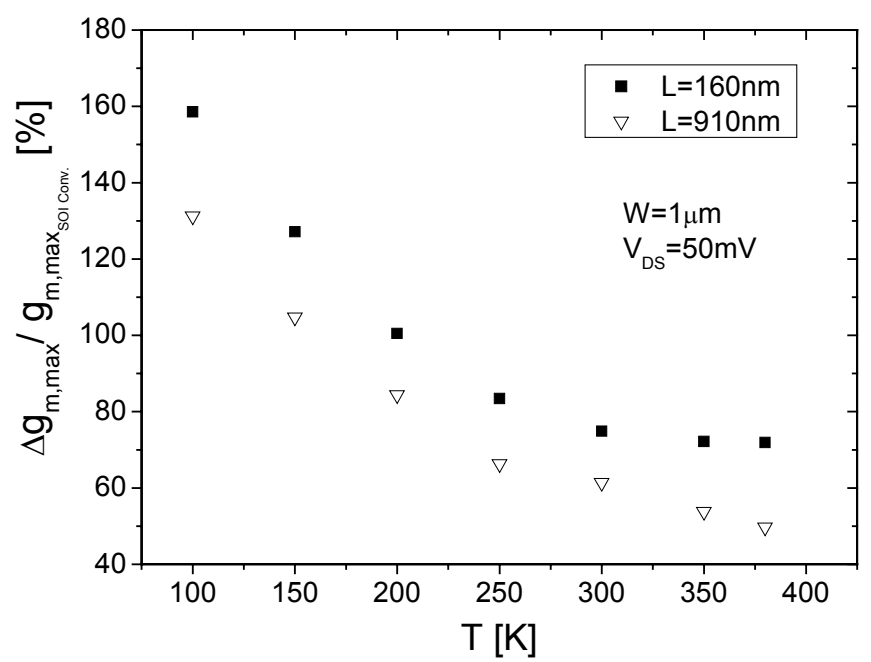

Figura 77 - Ganho de transcondutância proporcionado pelo sCESL, em relação ao SOI convencional, em função da temperatura, para dispositivos com $L=160 \mathrm{~nm}$ e $910 \mathrm{~nm}$.

O maior ganho de mobilidade obtido com a redução da temperatura está relacionado aos diferentes coeficientes de dilatação térmica do silício e do nitreto de silício (sCESL). O nitreto de silício apresenta coeficiente de dilatação térmica (aproximadamente $\left.3,3 \times 10^{-6} \mathrm{~K}^{-1}\right)$ maior que o do silício $\left(3 \times 10^{-6} \mathrm{~K}^{-1}\right)^{149}$. Com a redução da temperatura, o nitreto sofre maior contração que o silício, induzindo maior stress tensor sobre o canal, aumentando a eficácia da aplicação de tensão mecânica.

Um parâmetro que é bastante influenciado pela redução da temperatura é a inclinação de sublimiar (S). Este parâmetro foi extraído a partir do inverso da inclinação de sublimiar das curvas $I_{D S}$ em função de $V_{G F}$ com baixo valor de $V_{D S}$ (50 $\mathrm{mV}$ ), conforme indicado pela eq. (23). Conforme esperado, observa-se uma redução de $S$ com a temperatura, como pode ser visto na Figura 78, que apresenta este parâmetro extraído para transistores com comprimento de canal de $160 \mathrm{~nm}$ em função da temperatura. Na mesma figura é indicado o limite teórico, calculado a partir da eq. (24), utilizando os parâmetros tecnológicos indicados anteriormente e desprezando a presença de armadilhas de interface. Embora os valores de inclinação de sublimiar obtidos sejam cerca de $10 \mathrm{mV}$ /década maiores que o limite teórico, não foram observadas diferenças significativas entre os resultados extraídos para os dispositivos convencional e com tensão mecânica uniaxial, indicando que o sCESL não exerce influência sobre a geração de armadilhas de interface, que causaria diferenças nos valores extraídos para temperaturas mais baixas. 


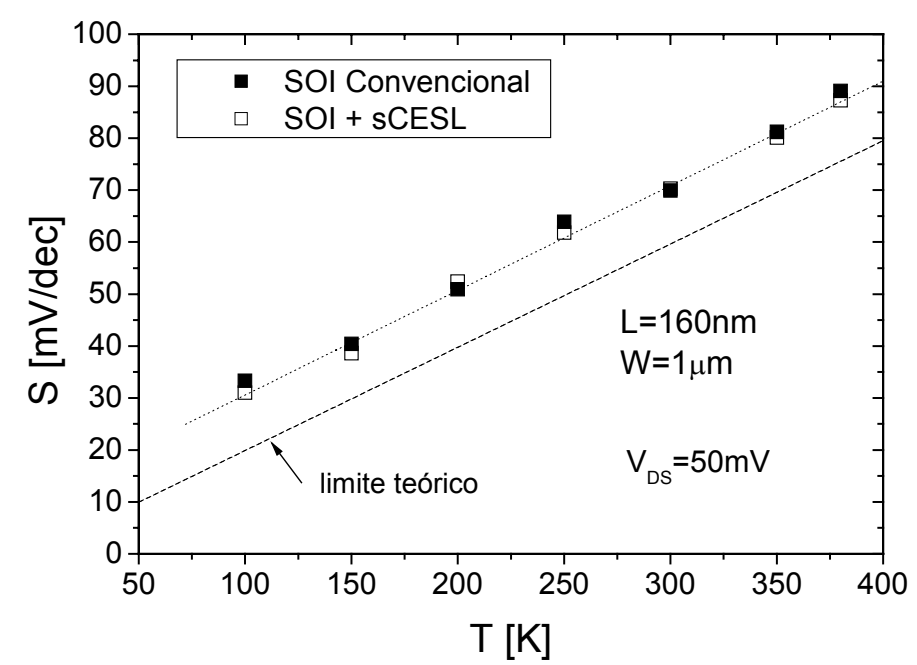

Figura 78 - Inclinação de sublimiar em função da temperatura para dispositivos com $\mathrm{L}=160 \mathrm{~nm}$.

\subsubsection{Características Analógicas de Transistores com Tensão Uniaxial}

A Figura 79 apresenta a transcondutância máxima $\left(g_{m, \max }\right)$ em saturação $\left(V_{D S}=0,55 \mathrm{~V}\right)$, em função da temperatura, para diferentes comprimentos de canal. $A$ partir desta figura, pode-se notar que o ganho de transcondutância proporcionado pela presença do SCESL, anteriormente observado em triodo, mantém-se em saturação, em todas as temperaturas estudadas.

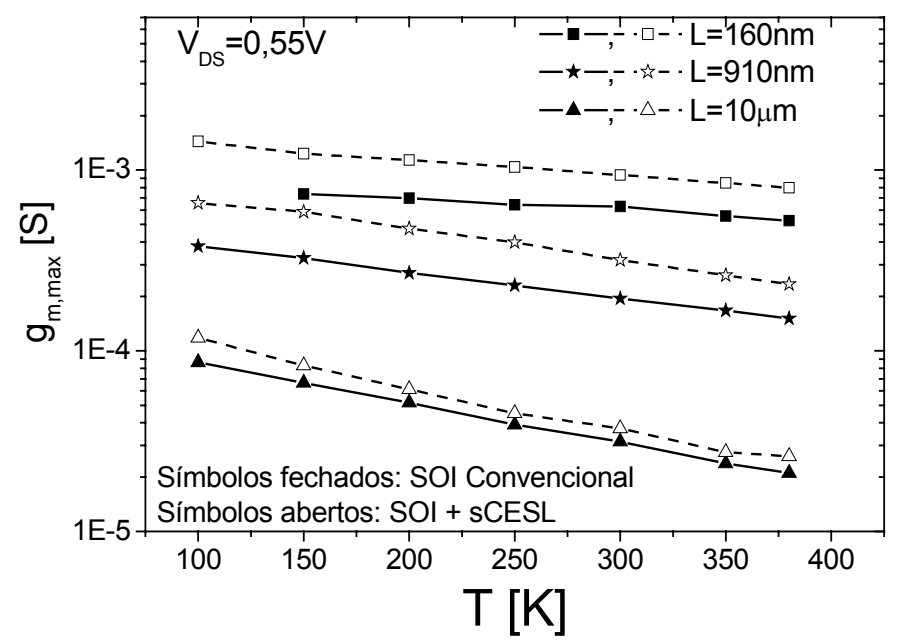

Figura 79 - Transcondutância máxima em função da temperatura, extraída para transistores com e sem $\mathrm{SCESL}$, com $\mathrm{V}_{\mathrm{DS}}=0,55 \mathrm{~V}$ para diferentes comprimentos de canal. 
Curvas da corrente de dreno em função da tensão de dreno ( $I_{D S}$ em função de $V_{\text {DS }}$ ) foram também medidas com sobretensão de condução variando entre 200 e 800 mV. A partir da diferenciação numérica destas curvas, a condutância de dreno foi obtida e os resultados para o transistor com $L=160 \mathrm{~nm}$ são apresentados na Figura 80 , todos em função da temperatura. A redução da temperatura causa a degradação de $g_{D}$ nos dois dispositivos, convencional e com SCESL. O dispositivo com tensão mecânica sofre maior degradação que o convencional, devido ao maior DIBL apresentado por estes transistores ${ }^{44}$, conforme será apresentado na próxima seção.

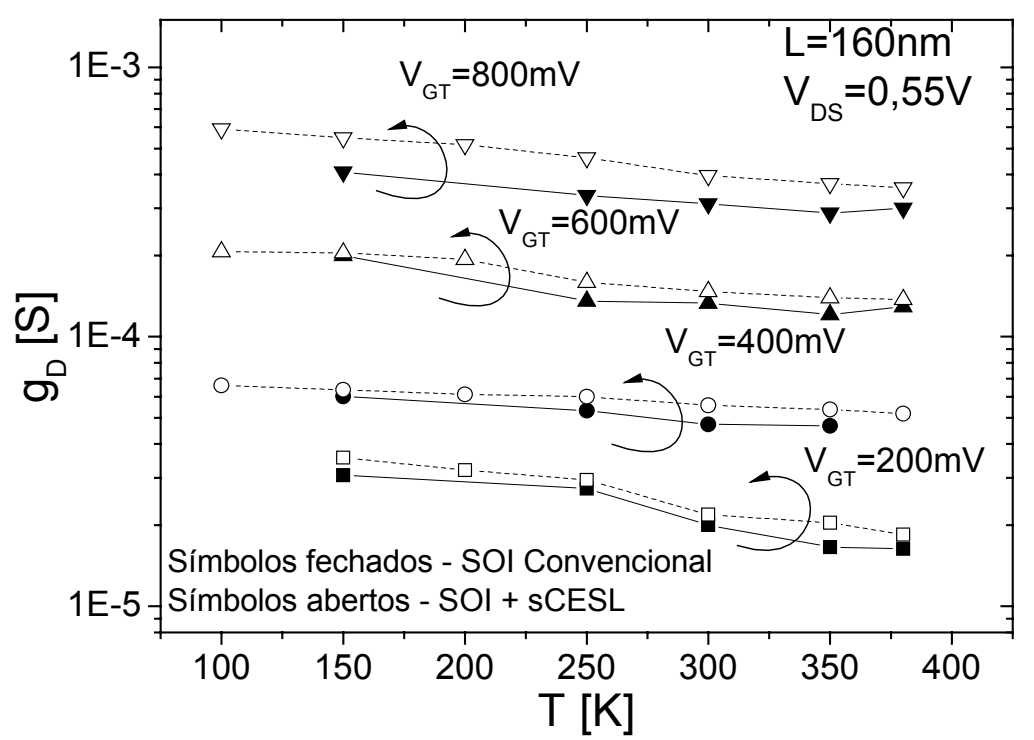

Figura 80 - Condutância de dreno em função da temperatura, extraída para dispositivos com $L=160 \mathrm{~nm}$, com $V_{D S}=0,55 V$ e diferentes valores de $V_{G T}$.

A partir da transcondutância e condutância de dreno, o ganho de tensão intrínseco foi extraído $\left(A_{v}=g_{m} / g_{D}\right)$ com $V_{D S}=0,55 \vee$ e $g_{m} / I_{D S}=3 V^{-1}$. Os resultados obtidos para $L=160 \mathrm{~nm}$, são apresentados na Figura 81, em função da temperatura. Os resultados apresentados permitem notar o aumento de $A_{V}$ com a redução da temperatura nos dois dispositivos. O maior ganho apresentado pelo dispositivo com sCESL deve-se, em parte, ao aumento da mobilidade. Além disto, a diminuição da temperatura faz com que menores valores de $\mathrm{V}_{\mathrm{GT}}$ sejam necessários para manter $\mathrm{g}_{\mathrm{m}} / \mathrm{I}_{\mathrm{DS}}$ constante (como pode ser visto no eixo à direita da Figura 81. Menores valores de $\mathrm{V}_{\mathrm{GT}}$ levam à redução de $\mathrm{g}_{\mathrm{D}}$ (como visto na Figura 80 ), contribuindo para o 
aumento de $A_{V}$. $O$ aumento de $A_{V}$ observado com a redução da temperatura é ligeiramente maior para o dispositivo convencional, devido à maior redução de $\mathrm{g}_{\mathrm{D}}$, relacionada à maior redução de $V_{G T}$ para uma dada temperatura (Figura 81).

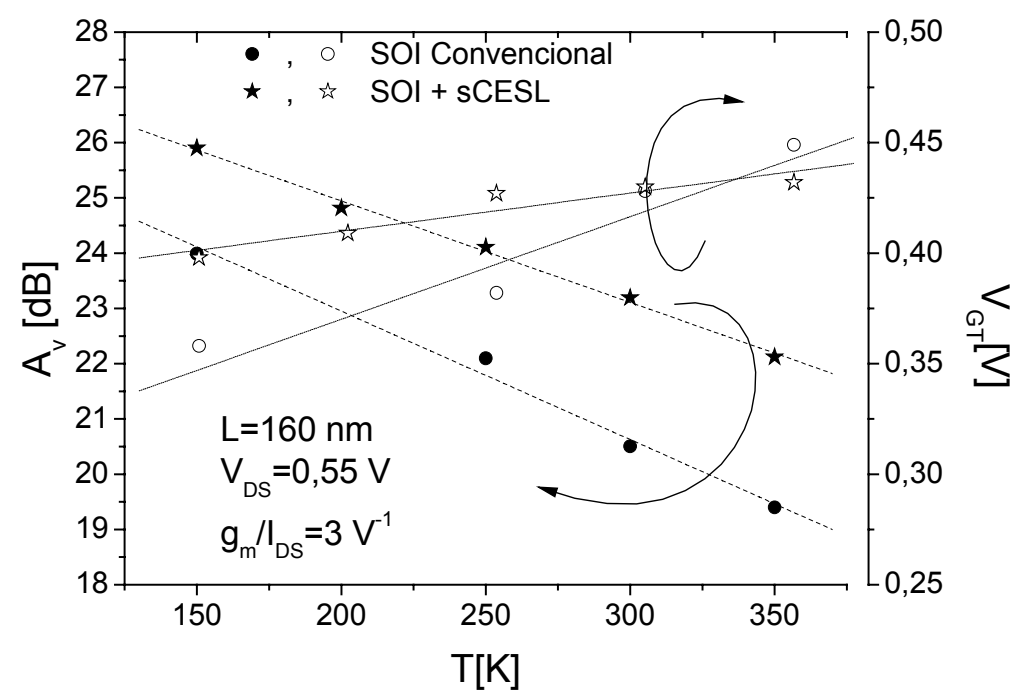

Figura 81 - Ganho de tensão intrínseco de dispositivos com $L=160 \mathrm{~nm}$, obtido com $V_{D S}=0,55 \vee$ e $g_{m} / l_{D S}=3 V^{-1}$, e $V_{G T}$ necessário, em função da temperatura.

A distorção harmônica também foi avaliada em função de redução da temperatura, tendo sido obtida através do método $\mathrm{IFM}^{82}$. Os resultados obtidos para dispositivos com $L=160 \mathrm{~nm}$ com $V_{D S}=0,55 \mathrm{~V}$ e $V a=50 \mathrm{mV}$ em função de $\mathrm{g}_{\mathrm{m}} / \mathrm{L}_{\mathrm{DS}}$ são apresentados na Figura 82. Como pode ser visto, o uso da tensão uniaxial faz com que o valor de $g_{m} / l_{D s}$ para $g_{m, \max }$ (relacionado ao ponto de distorção mínima) seja maior em baixas temperaturas. Para $\mathrm{g}_{\mathrm{m}} / \mathrm{l}_{\mathrm{DS}}$ acima do ponto de mínimo, o uso do SCESL tende a melhorar a linearidade com relação ao SOI convencional. Esta melhora, que é de cerca de $4 \mathrm{~dB}$ para $\mathrm{g}_{\mathrm{m}} / \mathrm{l}_{\mathrm{DS}}=5 \mathrm{~V}^{-1}$ em temperatura ambiente, aumenta com a redução da temperatura e chega a $8 \mathrm{~dB}$ em T=150 K e mesmo $\mathrm{g}_{\mathrm{m}} / \mathrm{l}_{\mathrm{DS}}$. Por outro lado, para valores de $\mathrm{g}_{\mathrm{m}} / \mathrm{l}_{\mathrm{DS}}$ abaixo do ponto de mínimo, observa-se linearidade inferior nos dispositivos com SCESL, devido à maior degradação da mobilidade nestes dispositivos ${ }^{150}$. Em relação ao HD3, observa-se valores menores que $-80 \mathrm{~dB}$ com e sem sCESL, para $\mathrm{g}_{\mathrm{m}} / \mathrm{L}_{\mathrm{DS}}>2 \mathrm{~V}^{-1}$, onde os dispositivos operam em saturação, que é a região de interesse para amplificadores. 

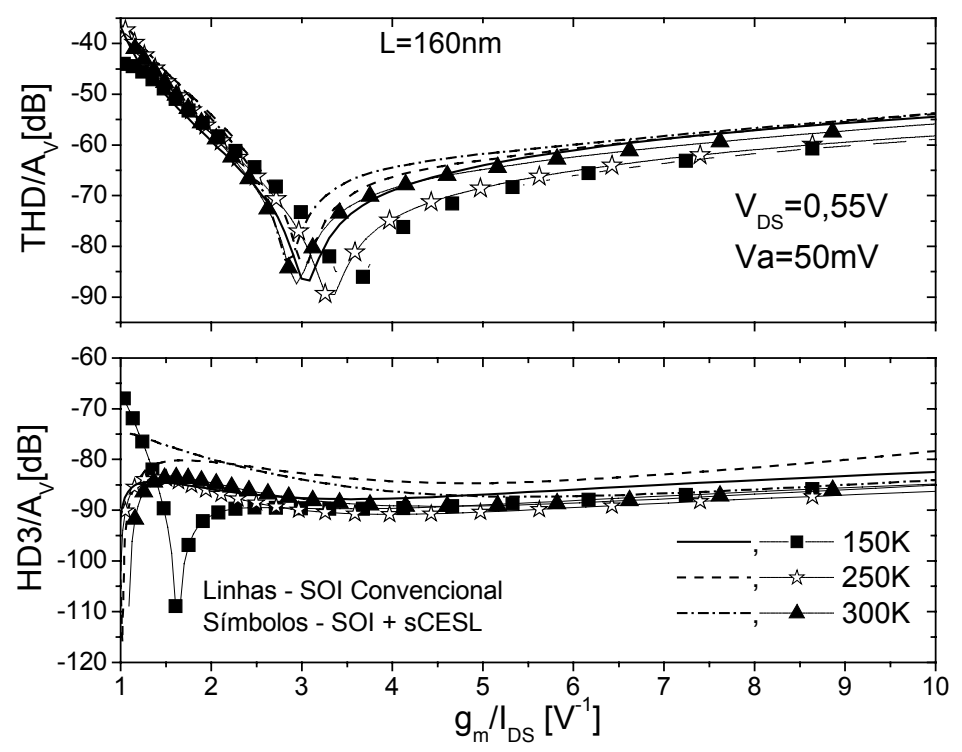

Figura $82-T H D / A_{v}$ e HD3/Av em função de $g_{m} / I_{D s}$ para dispositivos com $L=160 \mathrm{~nm}$ com $V_{D S}=0,55 \mathrm{~V}$ e $\mathrm{Va}=50 \mathrm{mV}$.

\subsection{COMPARAÇÃO ENTRE TRANSISTORES COM TENSÃO MECÂNICA UNIAXIAL E BIAXIAL EM BAIXAS TEMPERATURAS}

Nesta seção apresentamos uma comparação entre transistores com tensão uniaxial e biaxial. São apresentados parâmetros tais como $g_{m}, g_{D}, g_{m} / l_{D S}$, DIBL e ganho de tensão.

A Figura 83 apresenta os valores máximos da transcondutância $\left(g_{m, \max }\right)$ em função da temperatura, para transistores com $L=160 \mu \mathrm{m}$, convencional e com tensão mecânica, uniaxial e biaxial, extraídos com $V_{D S}=100 \mathrm{mV}$ e $550 \mathrm{mV}$. 


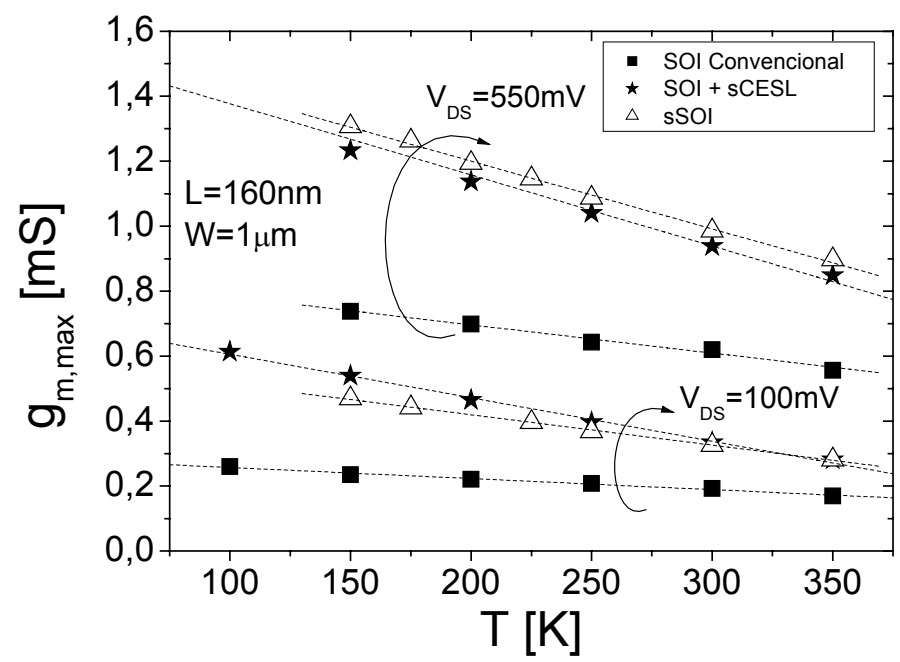

Figura 83 - Transcondutância máxima em função da temperatura, extraída com $V_{D S}=100 \mathrm{mV}$ e $550 \mathrm{mV}$ para transistores com e sem tensão mecânica $(\mathrm{L}=160 \mu \mathrm{m})$.

A partir das curvas apresentadas, verifica-se que o uso dos dois tipos de tensão mecânica promove $\circ$ aumento da transcondutância em todas as temperaturas. Além disso, a maior variação de $\mathrm{g}_{\mathrm{m} \text {,max }}$ com a temperatura indica que o ganho de transcondutância aumenta com a redução da temperatura para os dois tipos de tensão mecânica. Como mencionado anteriormente, no caso de transistores com SCESL, o maior ganho de transcondutância obtido com a redução da temperatura, está relacionado aos diferentes coeficientes de dilatação térmica do silício e do nitreto de silício. Quando a tensão biaxial é aplicada, o aumento do ganho de $g_{m, \max }$ com a redução da temperatura pode estar relacionado à redução da rugosidade superficial do silício tensionado em comparação com o convencional ${ }^{151}$, logo diminuindo a degradação de mobilidade.

Enquanto em regime linear a transcondutância tende a ser maior no dispositivo com SCESL, em saturação, com maiores valores de $V_{D S}$, a transcondutância máxima é maior no dispositivo sSOI. Esta inversão de comportamento pode ser atribuída à presença de impurezas neutras no material, aumentando o espalhamento de elétrons no transistor com sCESL ${ }^{150}$.

A relação entre a transcondutância e a corrente de dreno em função da corrente de dreno normalizada em inversão forte é apresentada na Figura 84. Como esperado, a redução da temperatura e conseqüente aumento da mobilidade, promove o aumento da relação $\mathrm{g}_{\mathrm{m}} / \mathrm{l}_{\mathrm{DS}}$ para todos os dispositivos (eq. (46)) ${ }^{152}$. Entretanto, devido ao maior ganho de mobilidade apresentado pelos transistores 
com tensão mecânica em baixa temperatura, o aumento de $g_{m} / l_{D S}$ é mais pronunciado nestes dispositivos. Entre os transistores com canal tensionado, o sSOI apresenta a relação $g_{m} / l_{D S}$ ligeiramente maior, também causado pela maior transcondutância em saturação (Figura 83). Além disso, pode-se notar que para um determinado valor de $\mathrm{g}_{\mathrm{m}} / \mathrm{I}_{\mathrm{DS}}$, transistores com $\mathrm{sCESL}$ ou sSOI fornecem maior corrente de dreno do que o convencional.

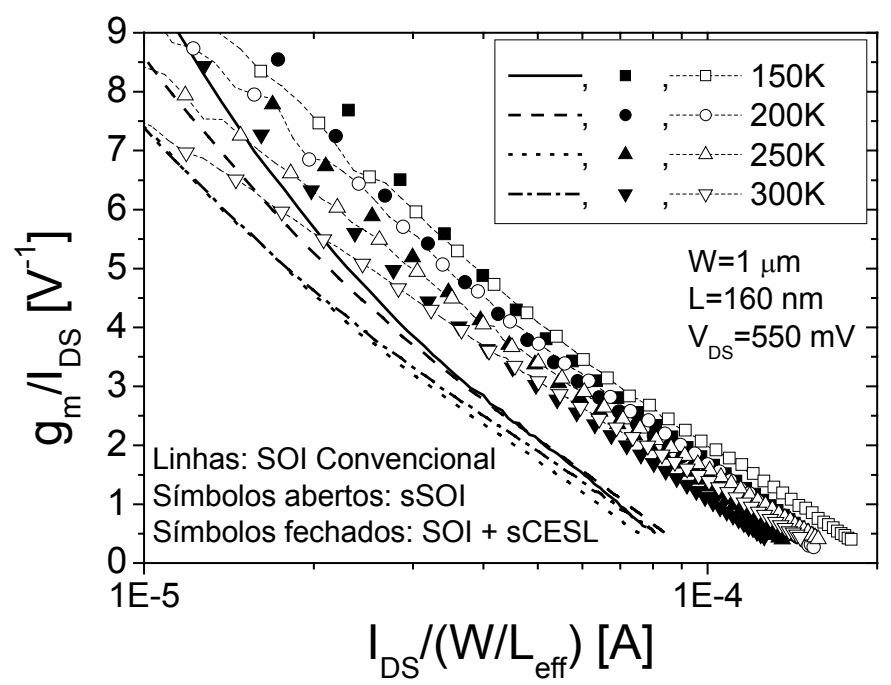

Figura 84 - Relação $\mathrm{g}_{\mathrm{m}} / \mathrm{I}_{\mathrm{DS}}$ em função da corrente de dreno normalizada, medida para transistores convencional e com tensão mecânica, com $L=160 \mathrm{~nm}$, em diversas temperaturas.

A partir das curvas $I_{D S}$ em função de $V_{D S}$ medidas com sobretensão de condução de $400 \mathrm{mV}$, foram extraídos os valores da condutância de dreno para os três transistores estudados. Os resultados em função da temperatura são apresentados na Figura 85.

A redução da temperatura causa a degradação da condutância de dreno em todos os transistores, com e sem tensão mecânica agindo sobre o canal. Além disso, pode-se verificar que a utilização da camada sCESL provoca uma pequena degradação de $g_{D}$ em relação ao transistor convencional. Por outro lado, a utilização de tensão biaxial (sSOI) tende a aumentar esta degradação. Este efeito está relacionado à piora do efeito $\mathrm{DIBL}$ nos transistores tensionados ${ }^{44}$. A fim de confirmar a influência da tensão mecânica sobre o DIBL, a Tabela 11 apresenta os valores do DIBL, extraídos para os três dispositivos em estudo. Para a definição do DIBL, a 
tensão de limiar foi extraída considerando nível de corrente constante e igual a $\mathrm{I}_{\mathrm{DS}}=10^{-7} \times(\mathrm{W} / \mathrm{L})[\mathrm{A}] \mathrm{com} \mathrm{V}_{\mathrm{DS}}=0,1 \mathrm{~V}$ e $\mathrm{V}_{\mathrm{DS}}=1 \mathrm{~V}$

Como pode ser visto a partir dos resultados apresentados, o DIBL diminui com a redução da temperatura para os todos os dispositivos, com e sem tensão mecânica. Entretanto, os dois tipos de transistores com canal tensionado são mais susceptíveis à ocorrência do DIBL, devido à maior degradação da tensão de limiar em saturação observada nestes dispositivos ${ }^{44}$. Além disso, entre os dois transistores com tensão mecânica, o transistor sSOI apresenta maior DIBL, em concordância com os resultados apresentados na ref. [44], e justificando a maior degradação de $g_{D}$ nestes transistores com a redução da temperatura.

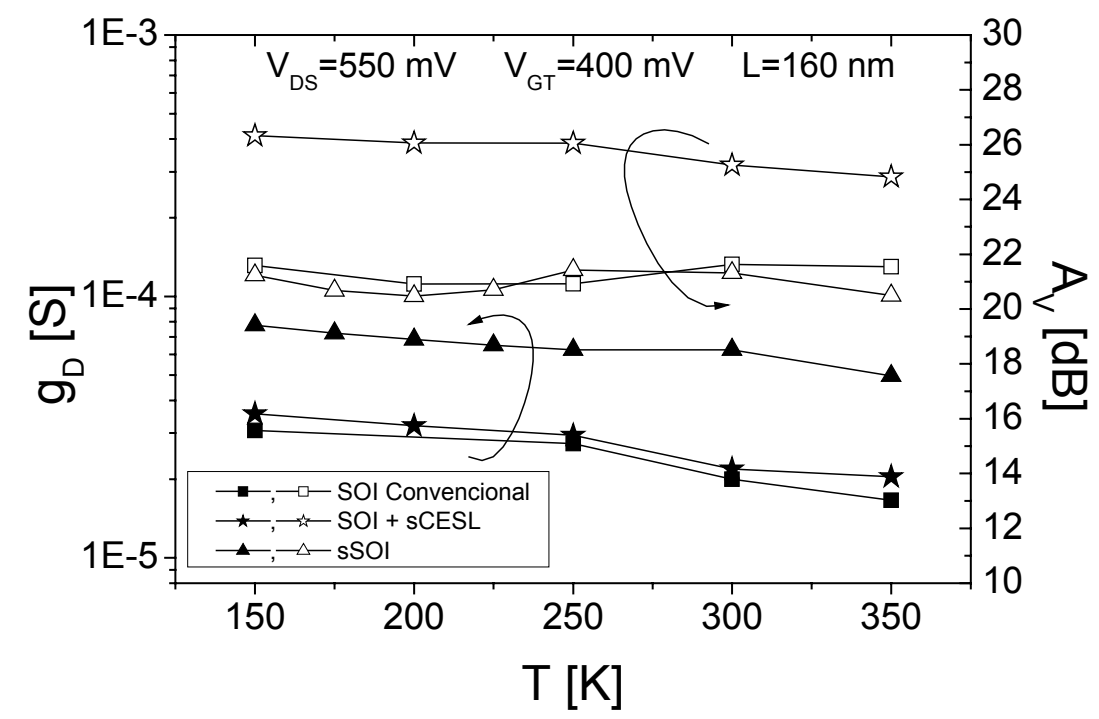

Figura 85 - Condutância de saída e ganho de tensão em função da temperatura, extraídos com $\mathrm{V}_{\mathrm{DS}}=550 \mathrm{mV}$ e $\mathrm{V}_{\mathrm{GT}}=400 \mathrm{mV}$.

Tabela 11 - DIBL extraído com $V_{D S}=100 \mathrm{mV}$ e $1 \mathrm{~V}$ para transistores com $\mathrm{L}=160 \mathrm{~nm}$ em diferentes temperaturas.

\begin{tabular}{cccc}
\hline \multirow{2}{*}{ Transistor } & \multicolumn{3}{c}{ DIBL [mV/V] } \\
\cline { 2 - 4 } & $\mathbf{1 5 0} \mathbf{~ K}$ & $\mathbf{2 0 0} \mathbf{~ K}$ & $\mathbf{3 0 0} \mathbf{~ K}$ \\
\hline Convencional & 20,55 & 31,11 & 39,28 \\
\hline SOI + sCESL & 40,44 & 46,00 & 56,97 \\
\hline sSOI & 109,33 & 123,33 & 145,57 \\
\hline
\end{tabular}


Na Figura 85 são também apresentados os valores do ganho intrínseco de tensão $\left(A_{V}=g_{m} / g_{D}\right)$ em função da temperatura, extraídos com $V_{D S}=0,55 \vee$ e $V_{G T}=400$ $\mathrm{mV}$. Estas curvas mostram que a redução da temperatura tende a aumentar ligeiramente o ganho de todos os transistores. Nos caso do transistor com tensão uniaxial, a pequena degradação de gD não é suficiente para degradar o ganho de tensão. Por outro lado, no caso do transistor sSOI, o aumento da transcondutância é compensado pela degradação da condutância de dreno, resultando em valores de $A_{V}$ semelhantes àqueles obtidos para o transistor convencional em todo o intervalo de temperaturas estudado.

SOUZA, M. de; PAVANELLO, M. A., MARTINO, J. A., SIMOEN, E., CLAEYS, C; Low temperature influence on the uniaxially strained FD SOI nMOSFETs behavior. Microelectronic Engineering, v. 84, p. 2121-2124, 2007.

SOUZA, M. de; PAVANELlO, M. A., MARTINO, J. A., SIMOEN, E., CLAEYS, C.; Analog Operation of Uniaxially Strained FD SOI nMOSFETs in Cryogenic Temperatures, 2007 IEEE SOI Conference Proceedings. v.1, p.45-46, 2007.

SOUZA, M. de, MARTINO, J. A., SIMOEN, E., CLAEYS, C., PAVANELLO, M. A. Analog Operation of Uniaxially and Biaxilally Strained FD SOI nMOSFETs at Cryogenics Temperatures. EuroSOI 2008 - Conference Proceedings, v. 1, p. 77-78, 2008. 


\section{CONCLUSÕES}

Apresentamos neste trabalho um estudo das características e aplicações analógicas de transistores SOI convencionais, de canal gradual (GC SOI), e com tensão mecânica, operando em baixas temperaturas.

Inicialmente, foram propostas alterações no modelo analítico contínuo existente para transistores GC SOI, para permitir sua utilização em baixas temperaturas. Este modelo foi incluído em um simulador de circuitos e seus resultados confrontados com dados obtidos através de simulações bidimensionais de dispositivos, realizadas para temperaturas entre $100 \mathrm{~K}$ e $300 \mathrm{~K}$, validando sua implementação. Tomando como base este modelo, foram propostas expressões analíticas contínuas para a transcondutância e a condutância de dreno destes transistores, visando sua utilização como ferramenta de projeto de circuitos analógicos. $O$ erro máximo obtido na comparação do modelo com os experimentos foi de $11 \%$.

Resultados experimentais, simulações numéricas bidimensionais e o modelo analítico proposto foram utilizados para a análise de características importantes para o funcionamento de circuitos analógicos. A distorção harmônica foi analisada para dispositivos operando em saturação e foi demonstrado que a maior linearidade apresentada por transistores de canal gradual deve-se a maior transcondutância e reduzida condutância de dreno proporcionada por esta estrutura. Além disso, foi observado que a melhora que a estrutura GC SOI apresenta, do ponto de vista da linearidade, sobre o transistor SOI convencional, se mantém também à medida que a temperatura é reduzida.

Utilizando estruturas com conjuntos de transistores idênticos, foi possível realizar um estudo das propriedades de descasamento de transistores GC SOI em função da temperatura. Foram estudados o descasamento da tensão de limiar e da corrente de dreno, com o transistor polarizado em triodo e saturação. Resultados do modelo analítico e de simulações numéricas bidimensionais foram também utilizados, a fim de explicar as razões físicas responsáveis pelo aumento do descasamento causado pela presença da região menos dopada próxima ao dreno. Foi observado que o maior descasamento nestes transistores está relacionado a 
variações de processo na definição da região fracamente dopada do canal. Para dispositivos operando em saturação, em baixas temperaturas, foi observada um aumento de cerca de $2 \%$ no descasamento da corrente em inversão fraca.

Foi também realizado um estudo do desempenho de transistores de canal gradual configurados como amplificador dreno comum (seguidor de fonte). Os resultados experimentais, sustentados também através de simulações numéricas, mostram que a utilização de transistores GC SOI nesta configuração, permite obter seguidores de fonte com ganho de tensão praticamente independente da tensão de entrada e bastante próximo do limite teórico (ganho unitário), enquanto com transistores com canal uniformemente dopado, o ganho apresenta significativa degradação para baixos valores de tensão de entrada. A maior tensão de ruptura, menor condutância de dreno e maior transcondutância são também responsáveis pela redução da distorção harmônica de seguidores de fonte implementados com transistores GC SOI em todas as temperaturas estudadas, comparativamente ao implementado com dispositivos convencionais.

Resultados de simulações numéricas indicam que a utilização de transistores GC SOI como seguidor de fonte, além de promover a melhora do ganho e linearidade em comparação com o transistor convencional com mesmo comprimento total de canal, permite uma redução das dimensões, da ordem de 5 vezes, sem degradação do ganho ou aumento da distorção harmônica.

Os primeiros resultados experimentais da operação de espelhos de corrente, nas arquiteturas Wilson e Cascode, com transistores de canal gradual em baixas temperaturas, também indicam vantagens da sua utilização em relação ao transistor SOI convencional. Considerando uma determinada temperatura, a utilização de transistores de canal gradual para a implementação de espelhos de corrente apresenta vantagens em relação à utilização de transistores convencionais, dentre as quais pode-se citar a melhora da precisão de espelhamento, tornando-a mais próxima da unidade, o aumento da excursão de saída (devido à redução da tensão de saturação e aumento da tensão de ruptura) e resistência de saída cerca de 4 vezes maior.

A redução da temperatura tende a degradar as características de saída dos espelhos de corrente implementados com transistores SOI convencionais e de canal gradual. Foi observada uma redução da excursão de saída, causada pelo aumento da tensão de saturação e redução da tensão de ruptura, para todos os espelhos de 
corrente, nas duas arquiteturas. A resistência de saída também sofre degradação à medida que a temperatura é reduzida, devido ao aumento da condutância de saída dos transistores. Apesar da degradação das características, causada pela redução da temperatura, espelhos de corrente com transistores GC SOI ainda apresentam melhoras em relação àqueles implementados com transistores uniformemente dopados.

Foram também estudados transistores SOI com tensão mecânica agindo sobre o canal em função da temperatura. Os resultados obtidos indicam que o ganho de mobilidade proporcionado por esta estrutura tensionada tende a aumentar com a redução da temperatura. No caso do transistor com tensão uniaxial, esta melhora está relacionada aos diferentes coeficientes de dilatação térmica do silício e da camada de nitreto de silício, que amplifica o efeito tensor sobre o canal e, no caso do transistor com tensão biaxial, esta melhora pode estar relacionada à redução da rugosidade superficial do silício tensionado em comparação com o convencional.

Observando as características analógicas destes transistores, foi possível observar que a tensão mecânica sobre o canal, seja uniaxial ou biaxial, promove o aumento da relação $\mathrm{g}_{\mathrm{m}} / \mathrm{l}_{\mathrm{DS}}$ em relação aos dispositivos sem tensão mecânica. Entretanto, a condutância de dreno apresenta degradação nestes transistores em relação ao convencional com mesmas dimensões. Esta degradação está relacionada ao aumento do DIBL, que é mais pronunciado no transistor com tensão biaxial. Combinando-se estes resultados, observou-se um aumento do ganho de tensão no transistor com tensão uniaxial e, no caso do transistor com tensão biaxial, praticamente não houve alteração em relação ao ganho do transistor convencional, indicando que a degradação da condutância de dreno é suficiente para compensar o aumento de transcondutância proporcionado pela tensão mecânica.

Como uma proposta para seqüência imediata do trabalho podemos citar o aprofundamento do estudo dos espelhos de corrente em função da temperatura, avaliando, através de medidas experimentais e simulações numéricas e analíticas, parâmetros tais como o tempo de estabilização. Além disso, de posse do modelo implementado em um simulador de circuitos, e das equações propostas para a transcondutância e a condutância de dreno, pode-se otimizar o projeto destes espelhos de corrente, a fim de verificar uma potencial redução de área que pode ser obtida com a utilização de transistores de canal gradual. 
Além disso, pode-se interligar os blocos analógicos estudados até 0 momento, em uma única célula, a fim de verificar a melhora no desempenho obtida pela utilização de transistores GC SOI, visando circuitos mais complexos. Novas células analógicas, tais como amplificadores operacionais, podem também ser projetadas e simuladas, para posterior fabricação, com o intuito de ampliar o estudo da aplicação de transistores de canal gradual em circuitos analógicos.

Durante este trabalho foram dimensionados e fabricados amplificadores operacionais de transcondutância, os quais podem ser explorados em função da temperatura. Além disso, novas estruturas, incluindo 20 transistores idênticos, com canal gradual, convencional e não-dopado, foram projetadas, e permitirão o aprofundamento do estudo do descasamento de parâmetros elétricos destes transistores. 


\section{REFERÊNCIAS}

1 COLINGE, J.P. Silicon-On-Insulator Technology: Materials to VLSI. 3rd Ed. Massachusetts: Kluwer Academic Publishers, 2004.

${ }^{2}$ LERAY, J.L. et al. From substrate to VLSI: investigation of hardened SIMOX without epitaxy, for dose, dose rate and SEU phenomena. IEEE Transactions on Nuclear Science, vol.35, n.6, p.1355-1360, 1988.

${ }^{3}$ KRULL, W. A.; LEE, J. C. Demonstration of the benefits of SOI for high temperature operation. In: Proceedings of SOS/SOI Technology Workshop, p. 69, 1988.

${ }^{4}$ HEMMENT, P. L. F. The SOI Odyssey. Proceedings of the Silicon-on-insulator technology and devices, v. 5, p. 1-12, 2003.

${ }^{5}$ YOSHIMI, M. et al; Observation of mobility enhancement in ultrathin SOI MOSFETs. Electronics Letters, v.24, n.17, p.1078-1079, 1988.

6 YOUNG, K.K. Short-channel effect in fully depleted SOI MOSFETs. IEEE Transactions on Electron Devices, v.36, n.2, p.399-402, 1989.

${ }^{7}$ FOSSUM, J.G. et al. SOI design for competitive CMOS VLSI. IEEE Transactions on Electron Devices, v.37, n.3, p.724-729, 1990.

${ }^{8}$ COLINGE, J.P. Hot-electron effects in silicon-on-insulator n-channel MOSFETs. IEEE Transactions on Electron Devices, v.34, n.10, p.2173-2177, 1987.

${ }^{9} \mathrm{SU}$, L.T. et al; Hot-carrier effects in fully depleted SOI nMOSFETs. IEEE IEDM Technical Digest, p.349-352, 1992.

${ }^{10}$ REIMBOLD, G.; AUBERTON-HERVE, A.-J. Aging analysis of nMOS of a $1.3-\mu \mathrm{m}$ partially depleted SIMOX SOI technology comparison with a 1.3- $\mu \mathrm{m}$ bulk technology. IEEE Transactions on Electron Devices, v.40, n.2, p.364-370, 1993.

${ }^{11} \mathrm{CHOI}$, J. Y. ; FOSSUM, J. G. Analysis and control of floating-body bipolar effects in fully depleted submicrometer SOI MOSFETs. IEEE Transactions on Electron Devices, v. 38, n. 6, p.1384-1391, 1991.

12 PAVANELLO, M.A. et al; The graded-channel SOI MOSFET to alleviate the parasitic bipolar effects and improve the output characteristics. In: Silicon-onInsulator Technology and Devices 1999, Pennington: The Electrochemical Society, p.293-298, 1999.

${ }^{13}$ PAVANELLO, M.A. et al; Analog performance and application of graded-channel fully depleted SOI MOSFETs. Solid-State Electronics, v.44, n.7, p.1219-1222, 2000. 
14 GIMENEZ, S.P. et al; Design of operational transconductance amplifiers with improved gain by using graded-channel SOI nMOSFETs, Proceedings of 16th Symposium on Integrated Circuits and Systems Design, p.26-31, 2003.

${ }^{15}$ DEHAN, M.; RASKIN, J-P ; An Asymmetric Channel SOI nMOSFET for Improving DC and Microwave Characteristics, Solid-State Electronics, v. 46, n. 7, p. 1005-1011, 2002.

${ }^{16}$ KILCHYTSKA, V. et al; Influence of Device Engineering on the Analog and RF Performances of SOI MOSFETs. IEEE Transactions on Electron Devices, v.50, n.3, p.577-588, 2003.

17 KIRSCHMAN, R. K.; Low-Temperature Electronics, IEEE Circuits and Devices Magazine, vol. 6, n. 2, p. 12-24, 1990.

18 HAMMOUD, A. et al; Electronic Components and Circuits for Extreme Temperature Environments, Proceedings of the 2003 10th IEEE International Conference on Electronics, Circuits and Systems, vol. 1, p. 44-47, 2003.

19 RIM, K. et al; Strained Si CMOS (SS CMOS) technology: opportunities and challenges, Solid-State Electronics, v. 47, n. 7, p. 1133-1139, 2003.

${ }^{20}$ HOYT, J. L. et al; Strained silicon MOSFET technology, IEDM Digest of Technical Papers, p. 23-26, 2002.

${ }^{21}$ BUTI, T. N. et al. A new asymmetrical halo source GOLD drain (HS-GOLD) deep sub-half-micrometer n-MOSFET design for reliability and performance. IEEE Transactions on Electron Devices, v. 38, n. 8, p. 1757-1764, 1991.

${ }^{22}$ CHANG, C. P.Shallow Trench Isolation. Encyclopedia of Materials: Science and Technology, p. 8437-8444, 2008.

${ }^{23}$ EL-KAREH, B. et al; Silicon on insulator-an emerging high-leverage technology. IEEE Transactions on Components, Packaging, and Manufacturing Technology, v. 18, n. 1, p. 187-194, 1995.

${ }^{24}$ SZE, S.M.; Physics of semiconductor devices, $2^{\text {nd }}$ Ed. New York (EUA): John Wiley and Sons, 1981.

${ }^{25}$ KRISHNAN, S.; FOSSUM, J.G. Grasping SOI floating-body effects. IEEE Circuits and Devices Magazine, v.14, n.4, p. 32-37, 1998.

${ }^{26}$ HIROAKI, M. SOI Becomes Practicable. Seiko Epson: Watch Type IC with Partially Depleted SOI. Semiconductor World, v. 19, n. 2, p. 74-77, 2000. 
27 CURRAN, B. et al. Power-constrained high-frequency circuits for the IBM POWER6 microprocessor. IBM Journal of Research and Development, v. 51, n. 6, p. 715-731, 2007.

${ }^{28}$ http://www.eetimes.com/showArticle.jhtml?articlelD=196701745.

${ }^{29}$ SHAHIDI, G. G. SOI Technology for the GHz era. IBM Journal of Research and Development, v. 46, n. 2/3, p. 121-131, 2002.

${ }^{30}$ KISTLER, N.; WOO, J. Detailed characterization and analysis of the breakdown voltage in fully depleted SOI n-MOSFET's. IEEE Transactions on Electron Devices, v.41, n.7, p.1217-1221, 1994.

31 YOSHIMI, M. et al; Two-dimensional simulation and measurement of highperformance MOSFETs made on a very thin SOI film. IEEE Transactions on Electron Devices, v.36, n.3, p.493-503, 1989.

32 GROESENEKEN, G. et al; Temperature dependence of threshold voltage in thinfilm SOI MOSFETs. IEEE Electron Device Letters, v.11, n.8, p.329-331, 1990.

${ }^{33}$ CHUANG, C.-T. et al; SOI for digital CMOS VLSI: design considerations and advances. Proceedings of the IEEE, v. 86, n.4, p. 689-720, 1998.

${ }^{34}$ LIM, H.K.; FOSSUM, J.G. Threshold voltage of thin-film silicon-on-insulator (SOI) MOSFET's. IEEE Transactions on Electron Devices, v.30, p.1244-1251, 1983.

${ }^{35}$ FLANDRE, D. et al; Comparison of SOI versus bulk performances of CMOS micropower single-stage OTAs. Electronics Letters, v.30, n.23, p.1933-1934, 1994.

${ }^{36}$ LIM, H.K.; FOSSUM, J.G. Current-voltage characteristics of thin-film SOI MOSFET's in strong inversion. IEEE Transactions on Electron Devices, v.31, p.401408, 1984.

${ }^{37}$ ICHIKAWA, F. et al. Fully depleted SOI process and device technology for digital and RF applications. Solid-State Electronics, v. 48, n. 6, p. 999-1006, 2004.

${ }^{38}$ http://www.oki.com/en/press/2002/z01114e.html

${ }^{39} \mathrm{http}: / /$ www.emmicroelectronic.com/DetailNews.asp? IdNews=37

${ }^{40}$ ROCHETTE, F. et al. Experimental evidence and extraction of the electron mass variation in $\left[\begin{array}{lll}1 & 1 & 0\end{array}\right]$ uniaxially strained MOSFETs. Solid-State Electronics, v. 51, n. 1112, p. 1458-1465, 2007.

${ }^{41}$ NAYFEH, H. M. et al; Effect of tensile uniaxial stress on the electron transport properties of deep scaled FD-SOI n-type MOSFETs, IEEE Electron Device Letters, v. 27, n. 4, p. 288-290, 2006. 
${ }^{42}$ GALLON, C. et al; Mechanical and electrical analysis of strained liner effect in 35 $\mathrm{nm}$ fully depleted silicon-on-insulator devices with ultra thin silicon channels, Japanese Journal of Applied Physics, v. 45, n. 4B, p. 3058-3063, 2006.

${ }^{43}$ LAUER, I.; ANTONIADIS, D.; Enhancement of electron mobility in ultrathin-body silicon-on-insulator MOSFETs with uniaxial strain, IEEE Electron Device Letters, v. 26, n. 5, p. 314-316, 2005.

${ }^{44}$ AUGENDRE, E. et al; On the scalability of Source/Drain Current Enhancement in Thin Film sSOI; Proceedings of ESSDERC, p. 301-304, 2005.

${ }^{45} \mathrm{RADU}$, I. et al. sSOI fabrication by wafer bonding and layer splitting of thin SiGe virtual substrates. Materials Science and Engineering: B, v. 135, n. 3, p. 231-234, 2006.

${ }^{46} \mathrm{MIZUNO}$, T. et al; High-performance strained-SOI CMOS devices using thin film SiGe-on-insulator technology, IEEE Transactions on Electron Devices, v. 50, n. 4, p. 988-994, 2003.

${ }^{47}$ GUTIERREZ, E. A., DEEN, J.; CLAEYS, C. L.; Low Temperature Electronics: Physics, Devices, Circuits and Applications, Academic Press, 1991.

${ }^{48}$ SELBERHERR, S. MOS Device Modeling at 77K, IEEE Transactions on Electron Devices, v. 36, n. 8, pp. 1464-1474, 1989.

49 McKELVEY, J. P.; Solid State and Semiconductor Physics, Harper \& Row Publishers, pp. 300-302, 1966.

${ }^{50}$ COLINGE, J.P.; COLINGE, C.A. Physics of Semiconductor Devices. $1^{\text {st }}$ Ed. Massachusetts: Kluwer Academic Publishers, 2002.

${ }^{51}$ BREWS, J.R.; Subthreshold behavior of uniformly and nonuniformly doped longchannel MOSFET. IEEE Transactions on Electron Devices, v. 26, p. 1282-1291, 1979.

${ }^{52}$ COLINGE, J.P.; Advanced CMOS devices made in thin SOI films. Extended Abstracts of $5^{\text {th }}$ International Workshop on Future Electron Devices, Miyagi-Zao, Japão, pp. 105-112, 1988.

53 VEERARAGHAVAN, S.; FOSSUM, J.G. A. Short-channel effects in SOI MOSFETs. IEEE Transactions on Electron Devices, v.36, n. 3, p. 522-528, 1989.

${ }^{54}$ MULLER, R.S.; KAMINS, T.I. Device Electronics for Integrated Circuits, .WileyInterscience Publication, 1986. 
${ }^{55}$ VEERARAGHAVAN, S.; FOSSUM, J.G. A physical short-channel model for the thin-film SOI MOSFET applicable to device and circuit CAD. IEEE Transactions on Electron Devices, v.35, p. 1866-1875, 1988.

${ }^{56}$ ADAM, A. O. et al; Analytical threshold voltage model for ultrathin SOI MOSFETs including short-channel and and floating-body effects. IEEE Transaction on Electron Devices, v. 46, n. 4, p. 729-737, 1999.

${ }^{57}$ WOO, J. C. S.; PLUMMER, J. D.; Short-channel effects in MOSFET's at LiquidNitrogen Temperature. IEEE Transaction on Electron Devices, v. 33, n. 7, p. 10121019, 1986.

58 PAVANELLO, M. A. et al, Analysis of Temperature-Induced Saturation Threshold Voltage Degradation in Deep-Submicrometer Ultrathin SOI MOSFETs, IEEE Transaction on Electron Devices, v. 52, n. 10, p. 2236-2242, 2005.

$59 \mathrm{SAH}, \mathrm{C}$. T. et al; Effect of zinc impurity in silicon solar-cell efficiency, IEEE Transaction on Electron Devices, v. 28, n. 3, p. 304-313, 1981.

60 CONWELL, E.; WEIAAKOPF, V. F.; Theory of impurity scattering in semiconductors, Physics Review, v. 77, n. 3, p. 388-390, 1950.

${ }^{61}$ BROOKS, H.; Scattering by ionized impurities in semiconductors, Physics Review, v. 83 , p. 879-885, 1951.

${ }^{62}$ CAUGHEY, D. M.; THOMAS, R. E.; Carrier mobilities in silicon empirically related to doping and field, Proc. IEEE, v. 52, p. 2192-2193, 1967.

63 DORKEL, J. M.; LETURCQ, Ph.; Carrier mobilities in silicon semi-empirically related to temperature, doping and injection level, Solid-State Electronics, v.24, n 9, p.821-825, 1981.

${ }^{64}$ LI, S. S.; THURBER, W. R.; The dopant density and temperature dependence of electron mobility and resistivity in n-type silicon, Solid-State Electronics, v.20, p.609616, 1977.

65 YOSHIMI, M. Two-dimensional simulation and measurement of highperformanceMOSFETs made on a very thin SOI film. IEEE Transactions on Electron Devices, v. 36, n. 3, p. 493-503, 1989.

${ }^{66}$ CANALI, C. et al. Electron and hole drift velocity measurements in silicon and their relation to electric field and temperature, IEEE Transactions on Electron Devices, v. 22, p. 1045-1047, 1975.

67 YOUNG, K.K; BURNS, J.A. Avalanche-Induced Drain-Source Breakdown in Silicon-on-Insulator n-MOSFET's. IEEE Transactions on Electron Devices, v.35, n.4, p. 426-431, 1988. 
${ }^{68} \mathrm{KRANTI}$, A. et al; Laterally asymmetric channel engineering in fully depleted double gate SOI MOSFETs for high performance analog applications. Solid-State Electronics, v.48, n.6, p.947-959, 2004.

${ }^{69}$ RAIS, K. et al; Temperature dependence of substrate current in silicon CMOS devices, Electronics Letters, v. 29, p. 778, 1993.

${ }^{70}$ DING, Y.-F. et al; Numerical study of self-heating effects of small-size MOSFETs fabricated on silicon-on-aluminum nitride substrate. Nuclear Science and Techniques, v. 17, n. 1, p. 29-33, 2006.

${ }^{71} \mathrm{JOMAAH}$, J. et al; Analysis and modeling of self-heating effects in thin-film SOI MOSFETs as a function of temperature. Solid-States Electronics, v. 38, n.3. p. 615618, 1995.

${ }^{72}$ BRODSKY, J.S. et al. A physics-based, dynamic thermal impedance model for SOI MOSFET's.IEEE Transactions on Electron Devices, v. 44, n. 6, p. 957-964, 1997.

${ }^{73}$ BUTI, T. N. et al; A new asymmetrical halo source GOLD drain (HS-GOLD) deep sub-half-micrometer n-MOSFET design for reliability and performance, IEEE Trans on Electron Devices, v. 38, n. 8, p. 1757-1764, 1991.

${ }^{74}$ COLINGE, J.P. Conduction mechanisms in thin-film accumulation-mode SOI pchannel MOSFETs. IEEE Transactions on Electron Devices, v. 37, p. 718-723, 1990.

${ }^{75}$ SILVEIRA, F. et al; $A g_{m} / I_{D}$ based methodology for the design of CMOS analog circuits and its application to the synthesis of a silicon-on-insulator micropower OTA. IEEE Journal of Solid-State Circuits, v.31, n.9, p.1314-1319, 1996.

${ }^{76}$ VITTOZ, E.A. Low power design: ways to approach the limits. Digest of Technical Papers, 41st ISSCC, p.14-18, 1994.

${ }^{77}$ FLANDRE, D. et al; Modelling and application of fully depleted SOI MOSFETs for low voltage, low power analogue CMOS circuits, Solid-State Electronics, v.39, n. 4, p. 455-460, 1996.

${ }^{78}$ SEDRA, A. S.; SMITH, K. C. Microeletrônica. Prentice Hall, 2007.

${ }^{79}$ FRANÇA, J. E.; TSIVIDIS, Y.; Design of analog-digital VLSI circuits for telecommunications and signal processing, Prentice Hall, 1994.

${ }^{80}$ ADAN, A. et al; Linearity and low-noise performance of SOI MOSFETs for RF applications, IEEE Transactions on Electron Devices, v. 49, n. 5, p. 881-888, 2002. 
81 GROENEWOLD, G.; LUBBERS, W. J.; Systematic Distortion Analysis for MOSFET Integrators with Use of a New MOSFET Model, IEEE Transactions on Circuits and Systems, vol. 41, n. 9, p. 569-580, 1994.

82 CERDEIRA, A. et al; Integral function method for determination of nonlinear harmonic distortion. Solid-State Electronics, v. 48, p. 2225-2234, 2004.

${ }^{83}$ CERDEIRA, A. et al; New method for determination of harmonic distortion in SOI FD MOSFETs, Solid-State Electronics, v. 46, n. 1, p. 103-108, 2002.

${ }^{84}$ SANCHEZ, J.J.; HSUEH, K.K.; DEMASSA, T.A. Drain-engineered hot-electronresistant device structures: a review. IEEE Transactions Electron Devices, v.36, n.6, p.1125-1132, 1989.

${ }^{85}$ KISTLER, N. et al. Sub-quarter-micrometer CMOS on ultrathin (400 A) SOI. IEEE Electron Devices Letters, v.13, n.5, p.235-237, 1992.

86 JENG, M.-C. et al. The effects of source/drain on deep submicrometer device performance. IEEE Transactions on Electron Devices, v.37, n.11, p.2408-2410, 1990.

${ }^{87}$ PAVANELLO, M. A. et al; An asymmetric channel SOI nMOSFET for reducing parasitic effects and improving output characteristics, Electrochemical and SolidState Letters, v.1, p.50-52, 2000.

88 PAVANELLO, M. A. et al; Graded-channel Fully Depleted Silicon-On-Insulator nMOSFET for Reducing the Parasitic Bipolar Effects, Solid-State Electronics, v. 44, n. 6, p. 917-922, 2000.

${ }^{89}$ SANZ, M. T. et al. Self-cascode SOI versus graded-channel SOI MOS transistors. IEE Proceedinf of Circuits, Devices and Systems, v. 153, n. 5, p. 461- 465, 2006.

${ }^{90}$ RAJPUT, S. S.; JAMUAR, S. S.; Low voltage analog circuit design techniques. IEEE Circuits and Systems Magazine, v. 2, n. 1, p. 24-42, 2002.

${ }^{91}$ GEROSA, A.; NEVIANI, A.; Enhancing output voltage swing in low-voltage micropower OTA using self-cascode. Electronics Letters, v. 39, n. 8, p. 638-639, 2003.

92 LEE, H., LEUNG, K. N.; MOK, P. K. T. Low-Voltage Analog Circuit Techniques Using Bias-Current Re-Utilization, Self-Biasing and Signal Superposition. Proceedings of the IEEE Conference on Electron Devices and Solid-State Circuits, $p$. 533-536, 2005.

93 GIMENEZ, S. P. et al; Design of operational transconductance amplifiers with improved gain by using graded-channel SOI nMOSFETs, Proceedings of 16th Symposium on Integrated Circuits and Systems Design, p.26-31, 2003. 
${ }^{94}$ SANTOS, A. A.; FLANDRE, D.; PAVANELLO, M. A.; Impact of Graded-Channel SOI MOSFET Application on the Performance of Cascode and Wilson Current Mirrors. Microelectronics Technology and Devices - SBMicro2007. v. 9, p. 441-450, 2007.

${ }^{95}$ DEHAN, M.; RASKIN, J-P ; An Asymmetric Channel SOI nMOSFET for Improving DC and Microwave Characteristics, Solid-State Electronics, v. 46, n. 7, p. 1005-1011, 2002.

${ }^{96}$ KILCHYTSKA, V. et al; Influence of Device Engineering on the Analog and RF Performances of SOI MOSFETs. IEEE Transactions on Electron Devices, v.50, n.3, p.577-588, 2003.

${ }^{97}$ PAVANELLO, M. A. et al; Low temperature operation of graded-channel SOI nMOSFETs for analog applications, Proceedings of $5^{\text {th }}$ European Workshop on Low Temperature Electronics (WOLTE5), p. 23-26, 2002.

${ }^{98}$ PAVANELLO, M. A. et al; A physically based continuous analytical graded-channel SOI nMOSFET model for analog applications, Proc. of the 4th IEEE Int. Caracas Conference on Devices, Circuits and Systems, Aruba, p. D030-1 - D030-5, 2002.

${ }^{99}$ SOUZA, M. de; et al; A charge-based continuous model for Submicron GradedChannel nMOSFET for Analog Circuit Simulation, Solid-State Electronics, v. 49, n.10, p. 1683-1692, 2005.

100 IÑíGUEZ, B. et al; A Physically-Based $\mathrm{C}_{\alpha}$-Continuous Fully-Depleted SOI MOSFET Model for Analog Applications, IEEE Transaction on Electron Devices, $v$. 43, n 4, p. 568-575, 1996.

${ }^{101}$ ATLAS User's Manual, SILVACO (2007).

102 SOUZA, M. de; PAVANELLO, M.A., FLANDRE, D.; Submicrometer GradedChannel SOI MOSFET Modeling for Low Temperature Analog Circuit Design, Proc. of $7^{\text {th }}$ International Workshop on Low Temperature Electronics (WOLTE7), v. WPP264, p. 57-64, 2006.

${ }^{103}$ MatLab (Version 6.1.0450 Release 12.1), The MathWorks, Inc, 2001.

${ }^{104}$ MALEVILLE, C.; MAZURÉ, C. Smart-Cut ${ }^{\circ}$ technology: from $300 \mathrm{~mm}$ ultrathin SOI production to advanced engineered substrates. Solid-State Electronics, v. 48, n. 6, p. 1055-1063, 2004.

105 TERAO, A. et al. Measurement of threshold voltages of thin-film accumulationmode PMOS/SOI transistors. IEEE Electron Device Letters, v.12, p.682-684, 1991. 
106 IÑIGUEZ, B. et al. A Physically-Based $C_{\alpha}$-Continuous Fully-Depleted SOI MOSFET Model for Analog Applications, IEEE Transanctions On Electron Devices, v.43, n.4, p.568-575, 1996.

${ }^{107}$ MAES, W.; DE MEYER, K.; VAN OVERSTRAETEN, R.; Impact ionization in silicon: A review and update, Solid-State Electronics, v. 33, p. 705-718, 1990.

${ }^{108}$ Eldo circuit simulator, Mentor Graphics, U. S. A.

109 LIN, S-C.; KUO, J.B.; Closed-form analytical drain current model considering energy transport and self-heating for short-channel fully-depleted SOI NMOS devices with lightly-doped drain structure biased in strong inversion, IEEE Transaction On Electron Devices, v. 49, n. 12, p.2193-2203, 2002.

110 SOUZA, M. de; PAVANELLO, M.A.; Charge-Based continuous explicit equations for the Transconductance and Output conductance of Submicron Graded-Channel SOI MOSFET's, Microelectronics Technology and Devices - SBMicro2006, p; 217226, 2006.

111 SOUZA, M. de, PAVANELLO, M. A. Charge-Based Continuous Equations for the Transconductance and Output Conductance of Graded-Channel SOI MOSFETs. JICS - Journal of Integrated Circuits and Systems, v.2, p.104 - 110, 2007.

112 CERDEIRA, A. et al; Advantages of Graded-Channel SOI FD MOSFET for Applications as a Quasi-Linear Resistor, IEEE Trans on Electron Devices, v.52, n.5, pp.967-972, 2005.

${ }^{113}$ IC Station, Mentor Graphics, U. S. A.

114 SOUZA, M. de; et al; Graded-Channel SOI nMOSFET Model Valid for Harmonic Distortion Evaluation. Proc. $25^{\text {th }}$ International Conference on Microelectronis (MIEL 2006), v. 2, p. 509-512, 2006.

${ }^{115}$ FLANDRE, D. et al; Comparison of SOI versus bulk performances of CMOS micropower single-stage OTA's, Electronics Letters, v. 30, n.23, p. 1933-1934, 1994.

${ }^{116}$ PELGROM, M. J. et al; Matching properties of MOS transistors, IEEE Journal of Solid-State Circuits, v. 24, p. 1433-1439, 1989.

117 SOUZA, M. de ; FLANDRE, D.; PAVANELLO, M. A. Analysis of Matching in Graded-Channel SOI MOSFETs. Microelectronics Technology and Devices SBMicro 2007, v. 9. p. 323-332, 2007.

118 SOUZA, M. de; FLANDRE, D.; PAVANELLO, M. A. Study of Matching Properties of Graded-Channel SOI MOSFETs. JICS. Journal of Integrated Circuits and Systems, v. 3, p. 69-75, 2008. 
${ }^{119}$ CROON, J. A. et al; Physical modeling and prediction of the matching properties of MOSFETs. Proceeding of the $34^{\text {th }}$ European Solid-State Device Research Conference/2004, p. 193-196, 2004.

${ }^{120}$ CROON, J.A. et al; An easy-to-use mismatch model for the MOS transistor; IEEE Journal of Solid-State Circuits, v.37, p. 1056-1064, 2002.

${ }^{121}$ LAKER, K. R.; SANSEN, W. M. C.; Design of Analog Integrated Circuits and Systems, Mcgraw-Hill, Inc. 1994.

122 TERAO, A. et al; Measurement of threshold voltages of thin-film accumulationmode PMOS/SOI transistors; IEEE Electron Device Lett., v.12, p. 682-684, 1991.

${ }^{123}$ DIFRENZA, R.; LLINARES, P.; GHIBAUDO, G. The impact of short channel and quantum effects on the MOS transistor mismatch. Solid-State Electronics, v. 47, n. 7 , p. 1161-1165, 2003.

${ }^{124}$ GWOZIECKI, R. Et al; Optimization of Vth roll-off in MOSFET's with advanced channel architecture. Retrograde doping and pockets. IEEE Transactions on Electron Devices, v. 46, n. 7, p. 1551-61, 1999.

125 DIFRENZA, R.; LLINARES, P.; GHIBAUDO, G.;. A new model for the current factor mismatch in the MOS transistor; Solid-State Electronics, v.47, n.7, p. 11611171, 2003.

${ }^{126}$ PELGROM, M. J.; TUINHOUT, H. P.; VERTREGT, M.; Transistor matching in analog CMOS applications, International Electron Devices Meeting/1998, p. 915-918, 1998.

127 DIFRENZA, R. et al; Effect of substrate voltage and oxide thickness on NMOSFET matching characteristics for a $0.18 \mu \mathrm{m}$ CMOS technology; International Conference on Microelectronics Test Structures/2001, PV 14, p. 7-10, 2001.

${ }^{128}$ VANCAILLIE L. et al; MOSFET mismatch in weak/moderante inversion: model needs and implications for analog design, Proceedings of the European Solid-State Circuits Conference/2003, p. 671-674, 2003.

${ }^{129}$ VITTOZ, E.A. The design of high-performance analog circuits on digital CMOS chips. IEEE Journal of Solid-State Circuits, v. 20, p. 657-665, 1985.

130 FOSSUM, E. CMOS image sensors: Electronic camera on chip. IEDM Tech Dig., p.17-25, 1995.

131 AFZALIAN, A.; FLANDRE, D.; Modeling of the Bulk Versus SOI CMOS Performances for the Optimal Design of APS Circuits in Low-Power Low-Voltage Applications. IEEE Trans on Electron Devices, v. 50, p. 106-110, 2003. 
${ }^{132}$ RAZAVI, B. Design of Analog CMOS Integrated Circuits. McGraw-Hill; 2002.

133 SOUZA, M. de; FLANDRE, D.; PAVANELLO, M. A.; Advantages of GradedChannel SOI nMOSFETs for Application as Source-Follower Analog Buffer. Artigo aceito para publicação no periódico Solid-State Electronics, 2008.

${ }^{134}$ SOUZA, M. de; FLANDRE, D.; PAVANELLO, M. A.; Improved Source-Follower Buffer Implementation by Using Graded-Channel SOI nMOSFETs. EuroSOI 2008 Conference Proceedings, v. 1, p. 35-36, 2008.

135 CUNHA, A.I.A., SCHNEIDER M.C., GALUP-MONTORO C. An MOS transistor model for analog circuit design. IEEE J Solid- State Circuits, v. 33, p. 1510-1519, 1998.

${ }^{136}$ ENZ C.; KRUMMENACHER, F.; VITTOZ, E. A.; An analytical MOS transistor model valid in all regions of operation and dedicated to low-voltage and low-current applications. Analog Integr Circ and Signal Processing, v. 8, p. 83-114, 1995.

${ }^{137}$ SOUZA, M. de; et al. A Fully Analytical Continuous Model for Graded-channel SOI MOSFET for Analog Applications. Microelectronics Technology and Devices SBMicro 2004, v. PV200. p. 27-32, 2004.

${ }^{138}$ SANSEN W.; Distortion in Elementary Transistor Circuits. IEEE Trans on Circuits and Systems - II: Analog and Digital Signal Processing, v. 46, p. 315-325, 1999.

139 SOUZA, M. de; FLANDRE, D.; PAVANELLO, M. A. Channel Length Influence on the Performance of Source-Follower Buffers Implemented with Graded-Channel SOI nMOSFETs. Microelectronics Technology and Devices - SBMicro2008. v.14, p.263272, 2008.

140 SOUZA, M. de, FLANDRE, D., PAVANELlO, M. A. Low Temperature and Channel Engineering Influence on the Behavior of Analog Source-Follower Buffers Proceedings of the Eighth International Workshop on Low Temperature Electronics. v.1, p.32-33, 2008.

141 SHOUCAIR, F.S.; Analytical and experimental methods for Zero-temperaturecoefficient biasing of MOS transistors. Electron Letters, v. 25, n. 17, p. 1196-1198, 1989.

${ }^{142}$ PAVANELLO, M. A.; MARTINO, J. A.; FLANDRE, D. High performance current mirrors using graded-channel SOI nMOSFETs, in Silicon-On-Insulator Technology and Devices 2001, The Electrochemical Society Proceedings, p. 319-324, 2001.

${ }^{143}$ LAKSHMIKUMAR, K.R.; HADAWAY, R. A.; COPELAND, M.A.; Characterization and modeling of mismatch in MOS transistors for precision analog design. IEEE Journal of Solid-State Circuit. V. 21, n. 6, p.1057-1066, 1986. 
${ }^{144}$ FERREIRA, R. S. Caracterização elétrica de espelhos de corrente baseados em transistores GC SOI MOSFET em função da temperatura. Dissertação de mestrado - Escola Politécnica da Universidade de São Paulo.

${ }^{145}$ SOUZA, M. de; et al; Low temperature influence on the uniaxially strained FD SOI nMOSFETs behavior. Microelectronic Engineering, v. 84, p. 2121-2124, 2007.

${ }^{146}$ SOUZA, M. de; et al; Analog Operation of Uniaxially Strained FD SOI nMOSFETs in Cryogenic Temperatures, 2007 IEEE SOI Conference Proceedings. v.1, p.45-46, 2007.

${ }^{147}$ SOUZA, M. de, et al; Analog Operation of Uniaxially and Biaxilally Strained FD SOI nMOSFETs at Cryogenics Temperatures. EuroSOI 2008 - Conference Proceedings, v. 1, p. 77-78, 2008.

${ }^{148}$ ROMANJEK, K. et al; New approach for the gate current source-drain partition modeling in advanced MOSFETs, Solid-State Electronics, v.47, n.10, p. 1657-1661, 2003.

${ }^{149}$ ASKELAND, D.R.; The Science and Engineering of Materials, $2^{\text {nd }}$ Ed. Boston (EUA): Chapman \& Hall, 1989.

${ }^{150}$ ANDRIEU, F. et al; In-depth study of strained SGOI nMOSFETs down to $30 \mathrm{~nm}$ gate length. Proceedings of European Solid-State Device Research Conference, ESSDERC, p. 297-300, 2005.

${ }^{151}$ BONNO, O. et al; High-Field Electron Mobility in Biaxially-tensile Strained SOI: Low Temperature Measurement and Correlation with the Surface Morphology. 2007 Symposium On VLSI Technology Digest of Technical Papers, p. 134-135, 2007.

152 COLINGE, J.-P. et al.; Temperature effects on trigate SOI MOSFETs. IEEE Electron Device Letters. V. 27, n. 3, p. 172-174, 3006. 


\section{APÊNDICE A}

\section{Exemplo de arquivo de simulação ATLAS de um transistor de canal gradual em $\mathrm{T}=100 \mathrm{~K}$.}

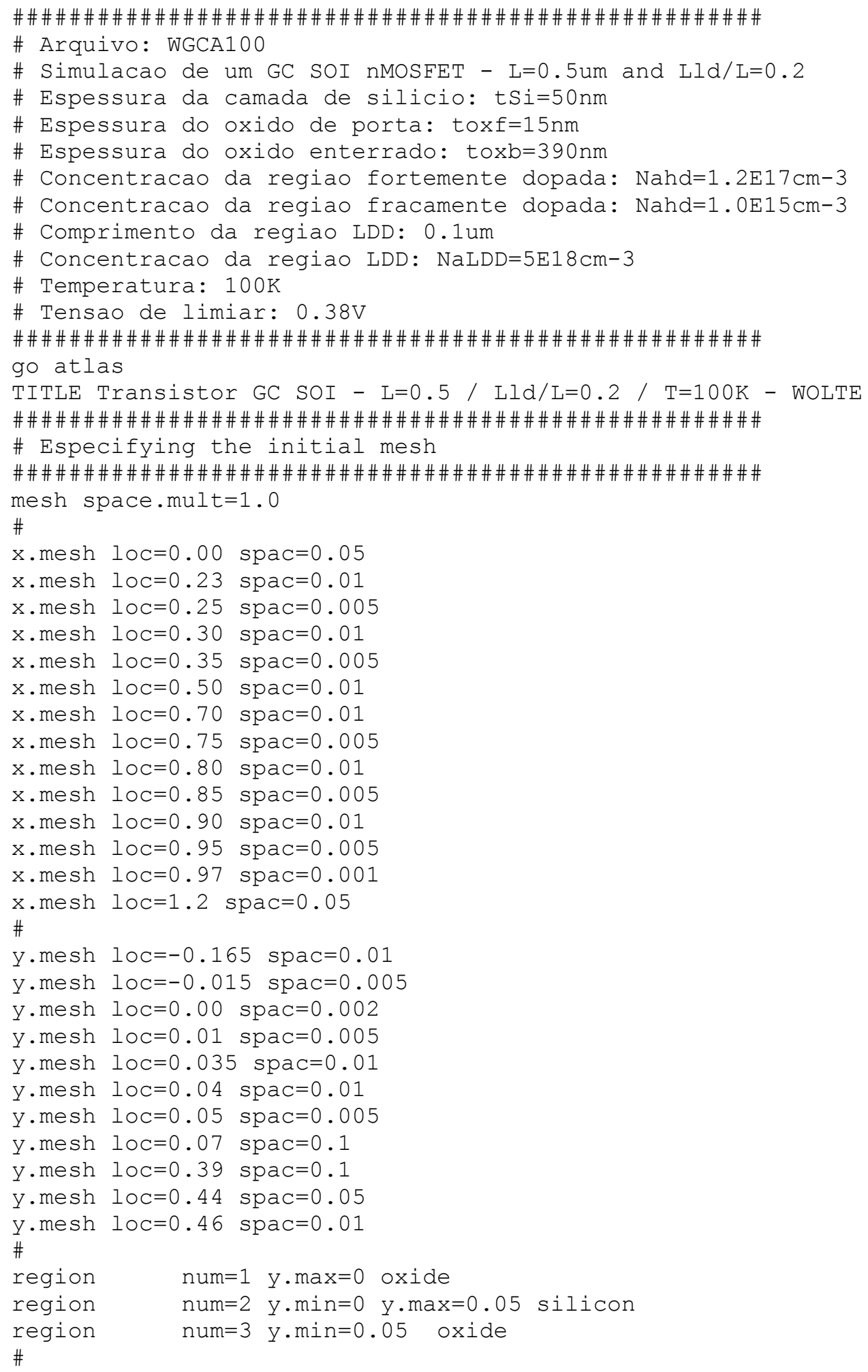




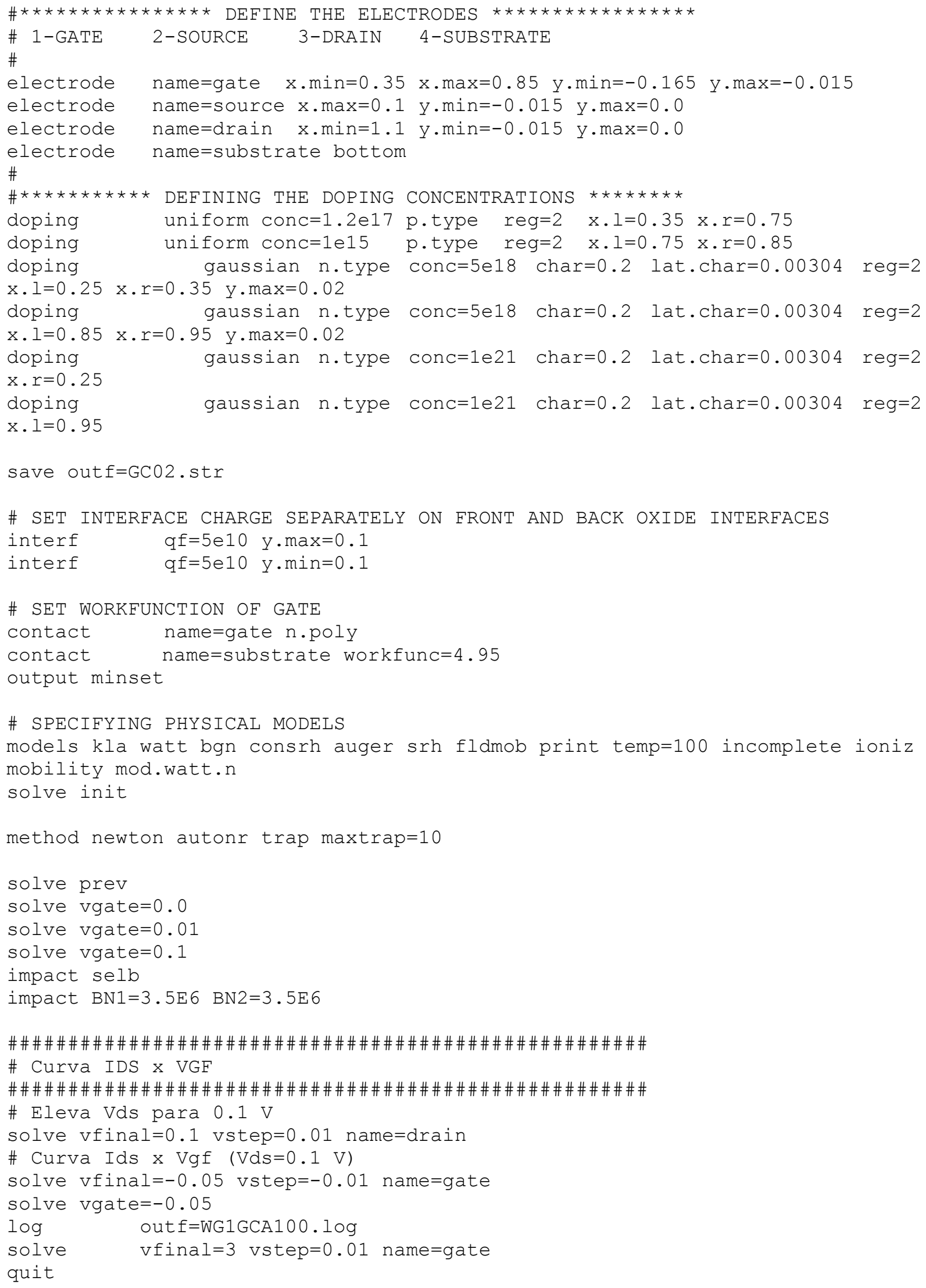




\section{APÊNDICE B}

Exemplo de arquivo de simulação ELDO de um transistor de canal gradual em $\mathrm{T}=100 \mathrm{~K}$.

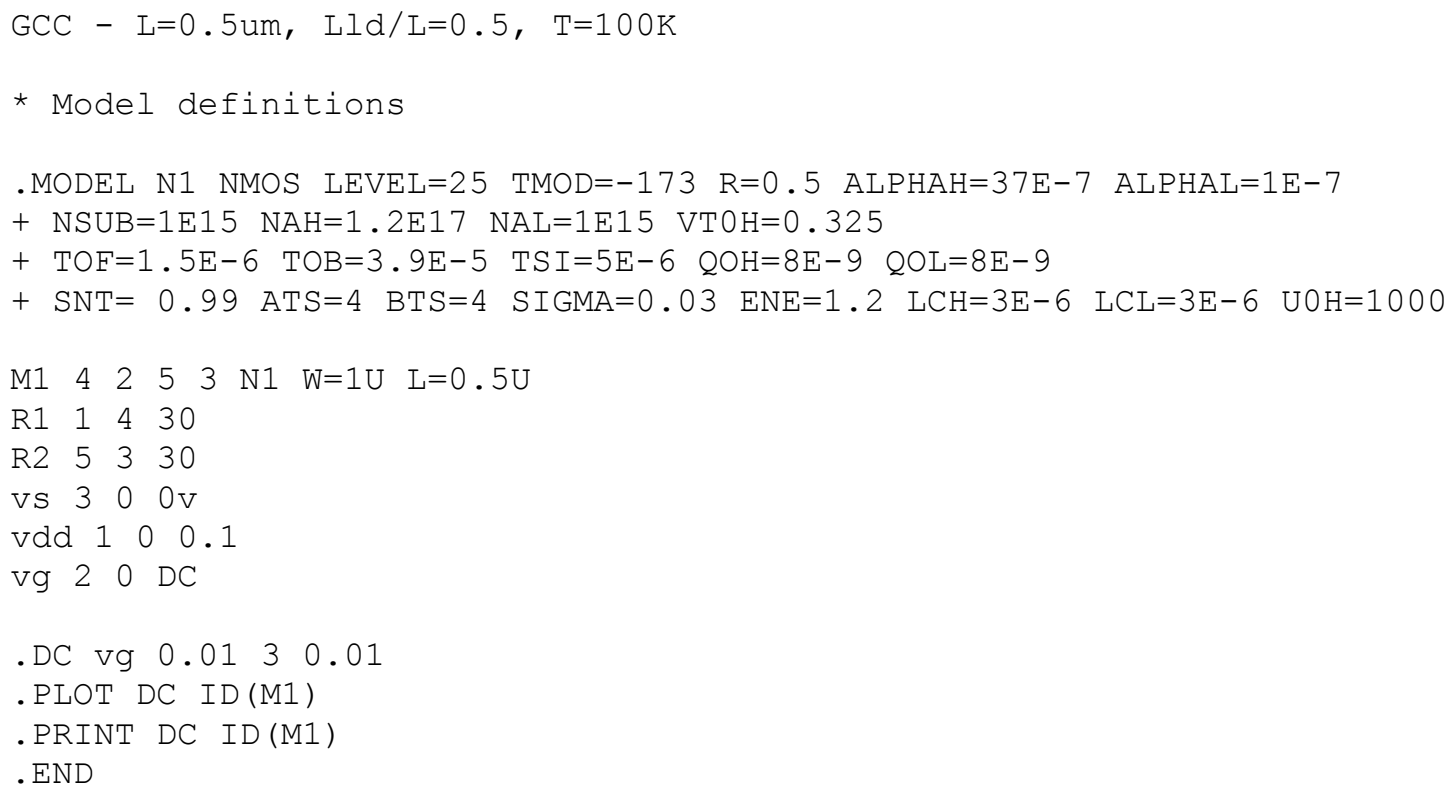




\section{APÊNDICE C}

\section{Exemplo de arquivo de simulação ATLAS de um seguidor de fonte implementado com transistor de canal gradual em $\mathrm{T}=300 \mathrm{~K}$.}

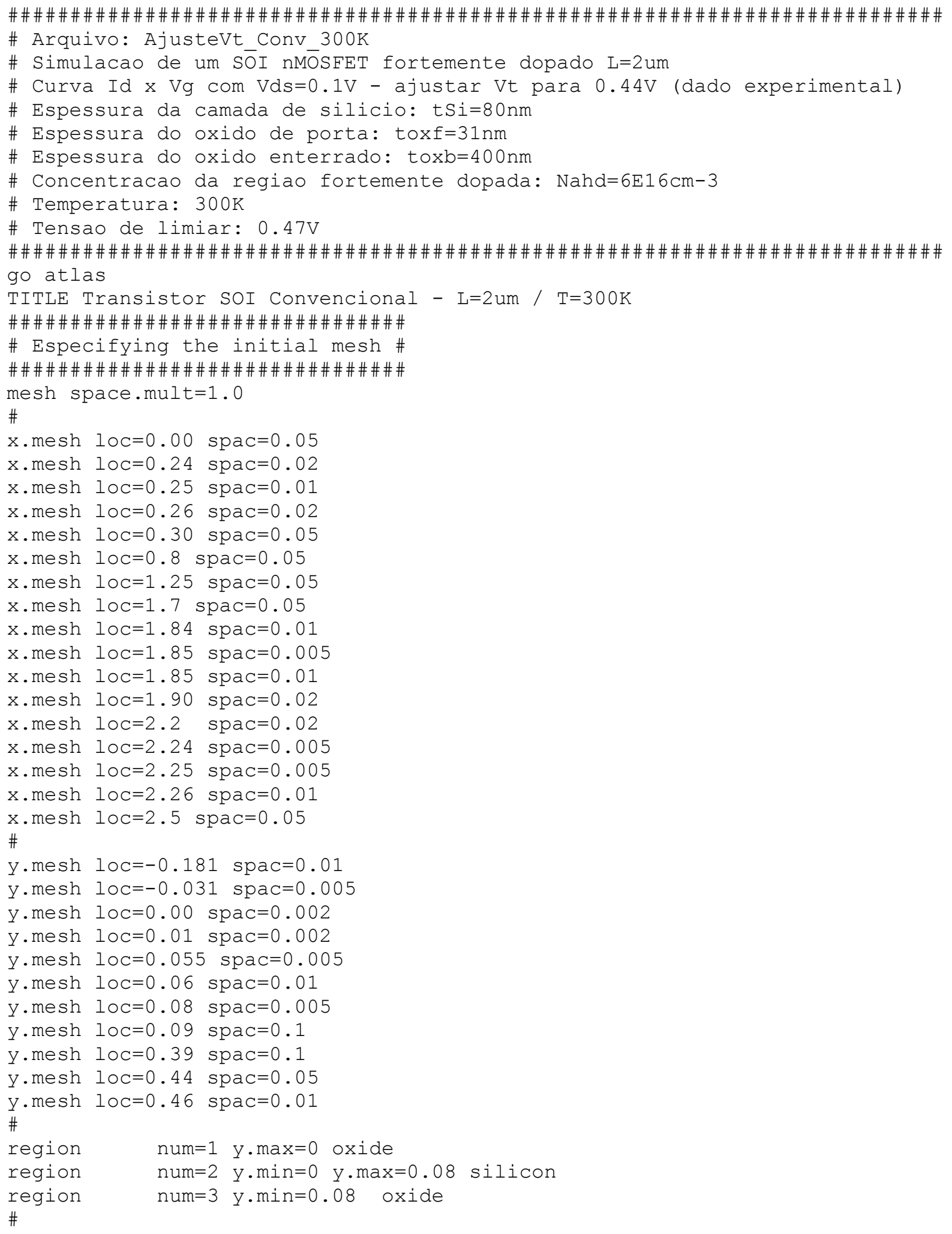




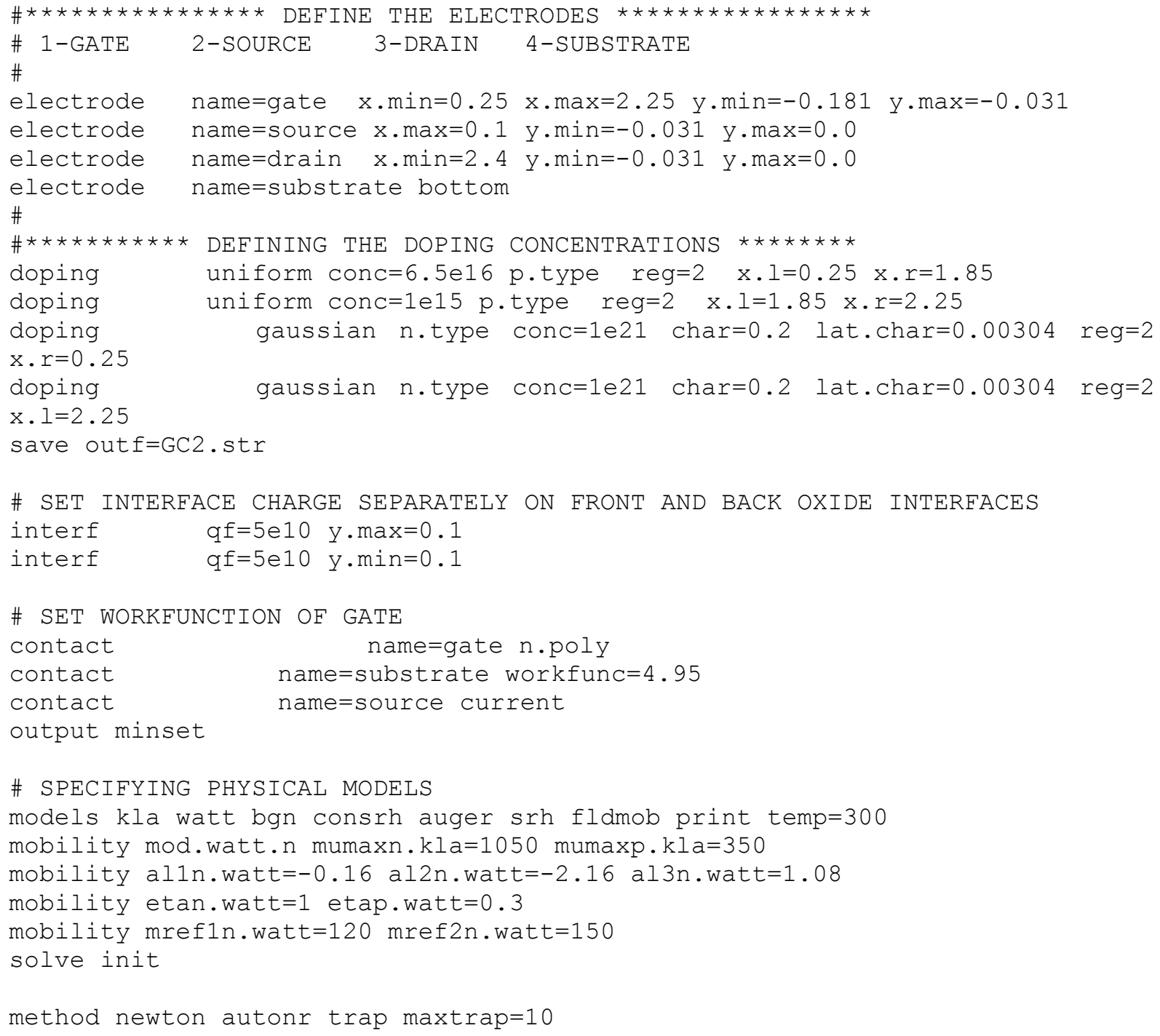


solve vfinal $=2.5$ vstep $=0.1$ name=drain

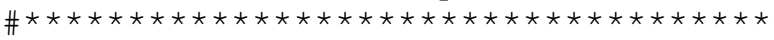

\# Sweep Vin

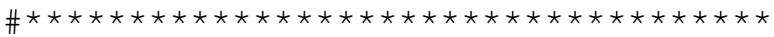

$\log$ out $\mathrm{f}=$ BB1AcomII.log

solve vfinal=2.5 vstep=0.01 name=gate

$\log$ off

solve vfinal=0.1 vstep=-0.1 name=gate

solve isource $=-1 \mathrm{E}-10$

solve isource $=-1 \mathrm{E}-9$

solve isource $=-1 \mathrm{E}-8$

solve isource $=-6.25 \mathrm{E}-8$

$\log$ outf=BB2AcomII.log

solve vfinal=2.5 vstep=0.01 name=gate

$\log$ off

solve vfinal=0.1 vstep=-0.1 name=gate

solve isource $=-1 \mathrm{E}-6$

solve isource $=-2.5 \mathrm{E}-6$

$\log$ outf=BB3AcomII.log

solve vfinal=2.5 vstep=0.01 name=gate

quit 